\title{
Acoustic emission evaluation of FRP composite specimens in tension and bending
}

\author{
Ryan Edward Arnold \\ West Virginia University
}

Follow this and additional works at: https://researchrepository.wvu.edu/etd

\section{Recommended Citation}

Arnold, Ryan Edward, "Acoustic emission evaluation of FRP composite specimens in tension and bending" (2003). Graduate Theses, Dissertations, and Problem Reports. 1342.

https://researchrepository.wvu.edu/etd/1342

This Thesis is protected by copyright and/or related rights. It has been brought to you by the The Research Repository @ WVU with permission from the rights-holder(s). You are free to use this Thesis in any way that is permitted by the copyright and related rights legislation that applies to your use. For other uses you must obtain permission from the rights-holder(s) directly, unless additional rights are indicated by a Creative Commons license in the record and/ or on the work itself. This Thesis has been accepted for inclusion in WVU Graduate Theses, Dissertations, and Problem Reports collection by an authorized administrator of The Research Repository @ WVU. For more information, please contact researchrepository@mail.wvu.edu. 


\title{
Acoustic Emission Evaluation of FRP Composite Specimens in Tension and Bending
}

\author{
by \\ Ryan Edward Arnold \\ Thesis submitted to the College of Engineering and Mineral Resources \\ at West Virginia University \\ in partial fulfillment of the requirements \\ for the degree of \\ Master of Science \\ in \\ Civil Engineering
}

\author{
Roger H. L. Chen, Ph.D.,Chair. \\ Hota GangaRao, Ph.D. \\ Eung Ha Cho, Ph.D.
}

Department of Civil and Environmental Engineering

Morgantown, West Virginia

2003

Keywords: Acoustic Emissions, Fiber Reinforced Polymer Composites, Neural Networks, Bridge Deck

Copyright 2003 Ryan E. Arnold 


\title{
Abstract \\ Acoustic Emission Evaluation of FRP Composite Specimens in Tension and Bending
}

\author{
Ryan E. Arnold
}

Fiber Reinforced Polymer (FRP) composite bridge decks are relatively new to the civil construction industry. Among their many advantages are corrosion resistance, low self-weight, stiffness-to-weight ratio, and high strength. One of the challenges to the greater usage of FRP structures is the lack of a nondestructive method to accurately evaluate its integrity while it is in place. Many typical methods of evaluating structures are ineffective on FRP composite structures due to their complex nature. In this study, nondestructive evaluation of FRP composite structures using Acoustic Emissions (AE) was conducted. A total of seven preliminary tension test specimens were tested along with fifteen bending specimens from different FRP manufacturers. AE characteristics were collected and analyzed for all specimens. The tension specimens were hand-laid specimens produced at WVU and were tested in accordance with ASTM D 3039. The fifteen bending specimens were of three types. The first set was from a Bedford Reinforced Plastics Inc. deck section, the second from a Bedford Reinforced Plastics Inc. plate section of different architecture, and the third from a section of Creative Pultrusions Superdeck. These bending specimens were created and tested according to ASTM D 790 with the exception of a decreased loading speed to allow for ample AE signal collection.

$\mathrm{AE}$ parameters were analyzed for all tests, and connections were found between them and the loading quarter of the specimens. Connections were also found between these parameters and the damage zone area of the specimens. These patterns allowed for additional analysis using Neural Networks (NN). By concentrating the AE parameters' patterns, NN were used to predict the loading quarter and damage zone of each specimen.

The connections between structural status and AE parameters found in this study can be translated to larger scale structures and on-site structures. This along with the NN prediction capabilities found can be used in the future to create a user-friendly, real-time health monitoring system for on-site structures. 


\section{Acknowledgements}

I would like to thank my adviser, Dr. Roger H. L. Chen for providing me with this opportunity and for his guidance and knowledge throughout its progression. I would also like to express my appreciation to my Committee members, Dr. Hota GangaRao and Dr. Eung Ha Cho, for their help throughout this project and for their reviewing of this work.

Appreciation is extended to the USDOT/FHWA for the financial support (project\# DTFH61-01-C-00002) for this work under the project Center of Excellence-Characteristics, Evaluation, and Implementation of Fiber Reinforced Polymer Composites for Highway Infrastructure. I would also like to thank the project monitor for this project.

I would also like to thank the Constructed Facilities Center and all of the assistants there. I would also like to say thank you to Mr. Dana Humberson for his help throughout this work, and Mr. Douglas Arnold for his great assistance. I would also like to thank Dr. Darrell Dean for allowing me to be his Teaching Assistant.

A special note of appreciation goes to Mr. Jeong-Hoon Choi for his great help throughout this study. I would also like to thank my office mates Mr. Joseph Sweet, Dr. Alejandro C. Kiriakidis L., Mr. Josh Cook, and Mr. Tuan-Chun Fu. A thank you also goes to Mr. Shu-Kai Yeh and Mr. Avinash Kaza for their assistance.

Last but certainly not least I would like to thank my family. This includes my parents Douglas and Dottie Arnold, and my little sister Jennifer Sue. I would also like to thank my grandparents Archie \& Lois Van Meter and Ollie Arnold, along with my extended family (Aunts, Uncles and Cousins).

This work also constitutes the final report for Task 2.4 of USDOT/FHWA project\# DTFH61-01-C-00002. 


\section{Table of Contents}

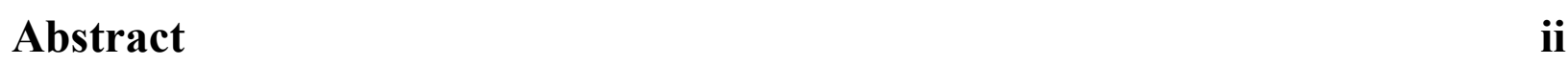

Acknowledgement

Table of Contents $\quad$ iv

$\begin{array}{ll}\text { List of Tables } & \text { vii }\end{array}$

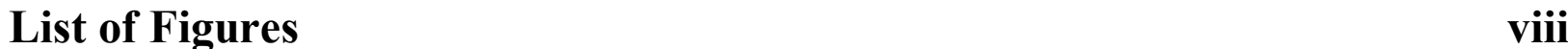

Chapter 1: Introduction 1

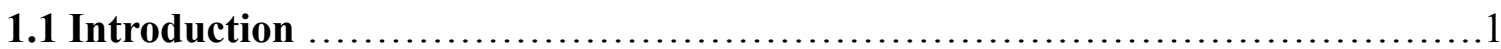

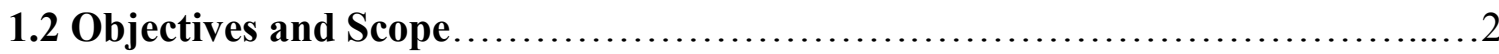

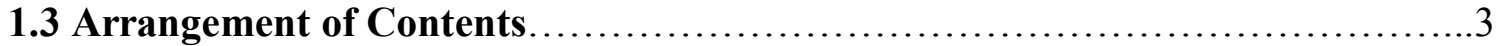

Chapter 2: Literature Review 4

2.1 FRP Composites.........................................................4

2.1.1 History and Background.........................................4

2.1.2 Advantages/Disadvantages.......................................... 5

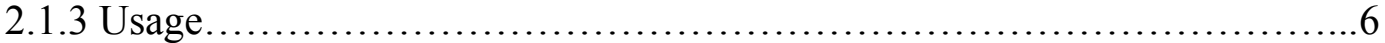

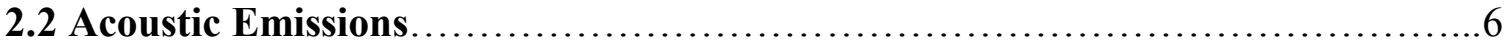

2.2.1 History and Background..............................................6

2.2.2 Important Terms.................................................... 8

2.2.3 Acoustic Emissions in FRP Materials.................................... 8

2.2.4 Wave Propagation.................................................. 9

2.2.5 Acoustic Emission Waveform Parameters............................... 10

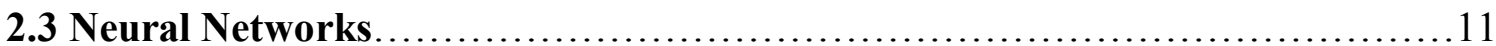

2.3.1 Background and History ............................................. 11

2.3.2 General Principles.................................................. 12

2.3.3 Current Uses...................................................... 13

2.4 Standards for AE Testing Techniques and FRP Composites.................13

$\begin{array}{ll}\text { Chapter 3: Tension Tests } & 17\end{array}$

3.1 Specimens............................................................ 17

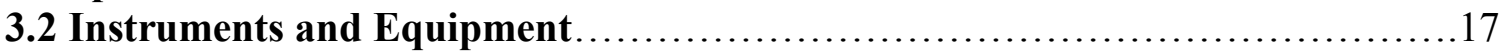

3.2.1 INSTRON Loading Frame......................................... 17

3.2.2 Computer Controller............................................... 18

3.2.3 AE Equipment and Software Setup ...................................18

3.2.4 Location Software and Setup......................................20

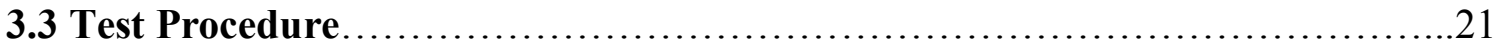

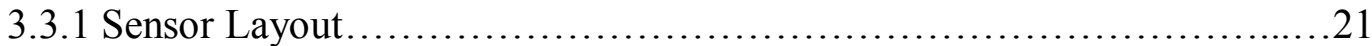

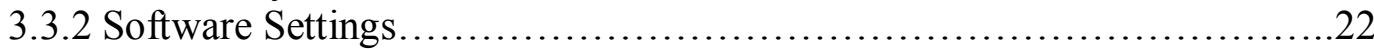


3.3.3 General Testing Procedure ..........................................23

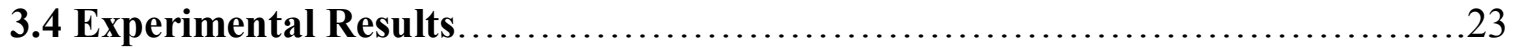

3.4.1 Frequency vs. Amplitude...........................................

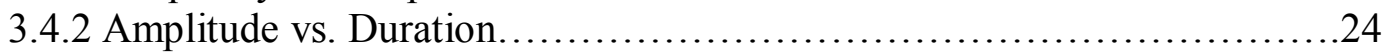

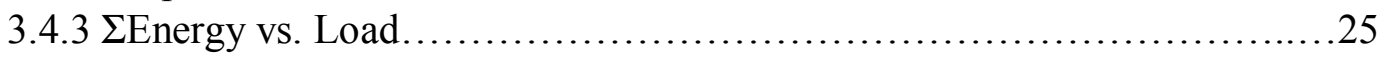

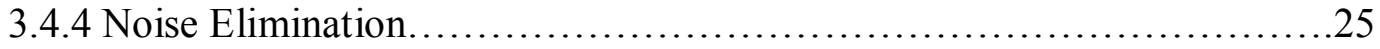

3.4.5 Location Plots/Damage Zone........................................27

Chapter 4: Bending Tests $\quad \mathbf{5 0}$

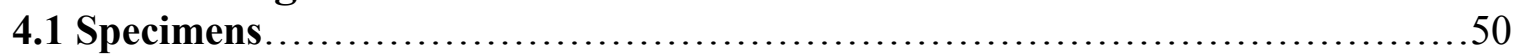

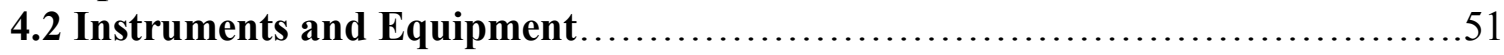

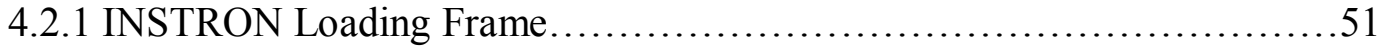

4.2.2 Computer Controller................................................

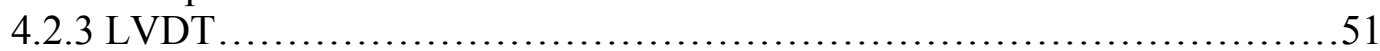

4.2.4 Acoustic Emission Equipment and Software Setup........................52

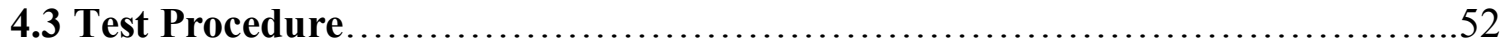

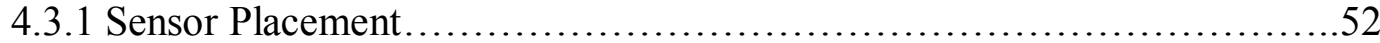

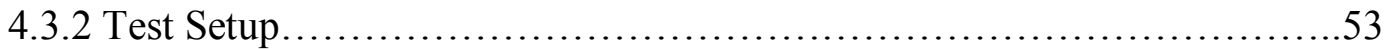

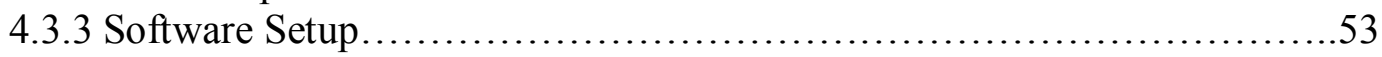

4.3.4 General Test Procedure.............................................53

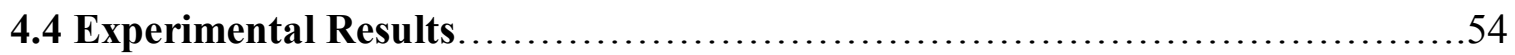

4.4.1 Specimen Failures................................................. 54

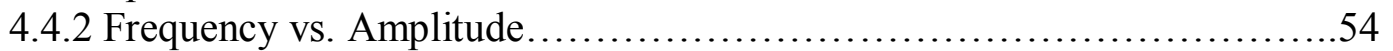

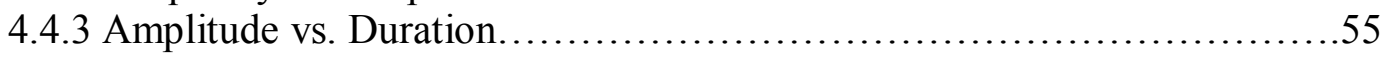

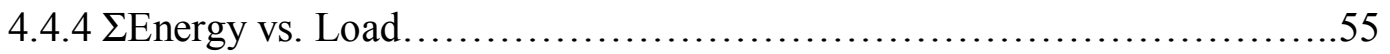

4.4.5 Load vs. Deflection.................................................56

Chapter 5: Neural Network Analysis of Tension Specimens 77

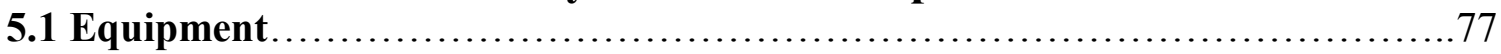

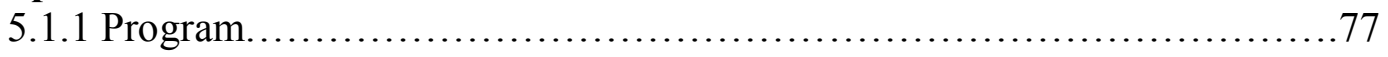

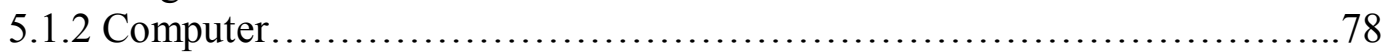

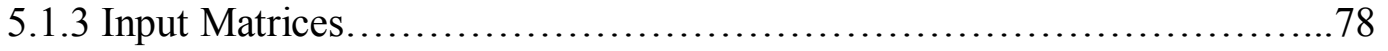

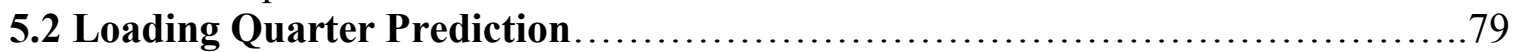

5.2.1 Training Set Matrices............................................. 79

5.2 .2 Test Set Matrices................................................... 81

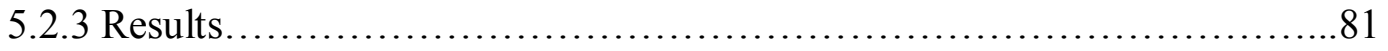

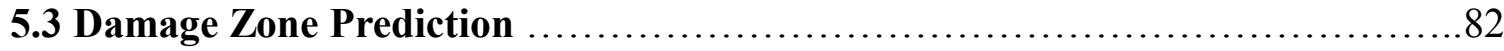

5.3.1 Training Set Matrices............................................. 82

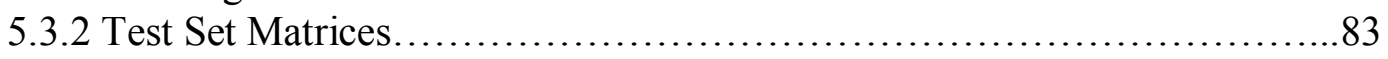

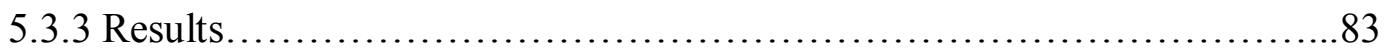

Chapter 6: Neural Network Analysis of Bending Specimens 97

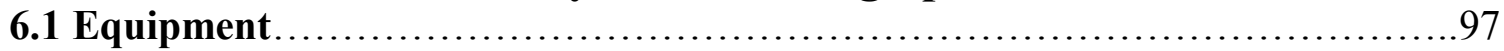

6.1.1 Input Matrices...................................................... 97

6.2 Loading Quarter Prediction............................................... 97 


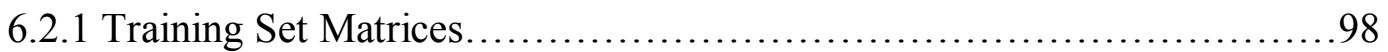

6.2.2 Test Set Matrices..................................................... 98

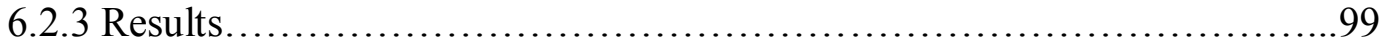

6.3 Damage Zone Prediction .............................................. 100

6.3.1 Training Set Matrices.............................................. 100

6.3.2 Test Set Matrices....................................................... 101

6.3 .3 Results.......................................................... 101

Chapter 7: Conclusions, Applications, \& Recommendations 126

7.1 Conclusions........................................................... 126

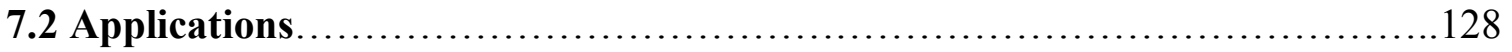

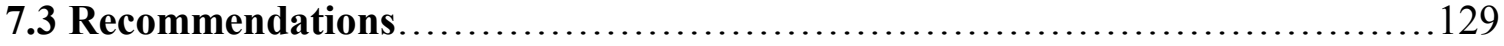

$\begin{array}{ll}\text { References } & 132\end{array}$

$\begin{array}{ll}\text { Appendix-A } & 135\end{array}$

Frequency vs. Amplitude Plots

Tension Specimens

Tests $2 \mathrm{t}-7 \mathrm{t}$

Appendix-B

142

Amplitude vs. Duration Plots

Tension Specimens

Tests $2 \mathrm{t}-7 \mathrm{t}$

Appendix-C

149

$\Sigma$ Energy vs. Load Plots

Tension Specimens

Tests $2 \mathrm{t}-4 \mathrm{t}, 6 \mathrm{t}-7 \mathrm{t}$

Appendix-D

Frequency vs. Amplitude Plots

Bending Specimens

Tests B1, B3-B5, B6, B8-B10, C1, C3-C5

Appendix-E

Amplitude vs. Duration Plots

Bending Specimens

Tests B1, B3-B5, B6, B8-B10, C1, C3-C5

Appendix-F

$\Sigma$ Energy vs. Load

Bending Tests

Tests B1, B3-B5, B6, B8-B10, C1, C3-C5

Appendix-G

Load vs. Deflection Plots

Bending Tests

Tests B2-B5, B6, B8-B9, C1-C3, C5

Vita 


\section{List of Tables}

Table 3.1 Wave Speed Calculation of Six-Layer Specimen.................................30

Table 3.2 Wave Speed Calculation of Four-Layer Specimen................................ 30

Table 4.1 Specimen Dimensions and Young's Modulus.....................................58

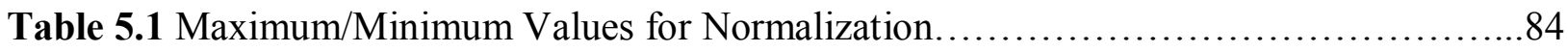

Table 5.2 Results for NN Load Quarter Predictor......................................... 85

Table 5.3 Results for NN Damage Zone Predictor ......................................... 86

Table 6.1 Minimum/Maximum Values for Normalization................................103

Table 6.2 Load Quarter Predictor Results, Specimens Separated.............................104

Table 6.3 Load Quarter Predictor Results, All Specimens Combined.........................105

Table 6.4 Wave Speed Calculation Summary............................................ 106

Table 6.5 Damage Zone Predictor Results, Specimens Separated............................107

Table 6.6 Damage Zone Predictor Results, All Specimens Combined.........................108 


\section{List of Figures}

Figure 2.1 Typical AE Waveform and Parameters...................................... 15

Figure 2.2 Typical Neural Network Architecture..................................... 16

Figure 3.1 INSTRON 8501 Loading Frame, Load Cell, and Actuator........................31

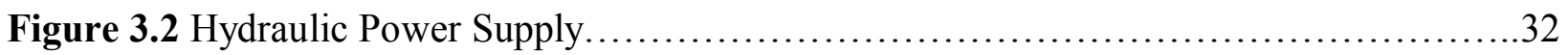

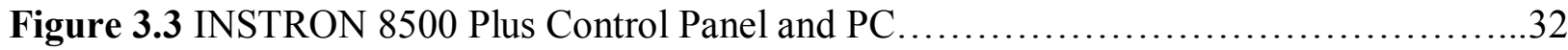

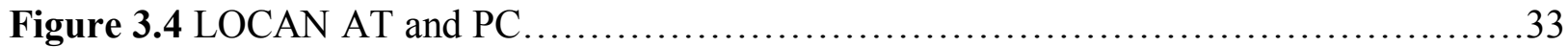

Figure 3.5 (a) PICO Sensor and (b) Model 1220A Preamplifier............................33

Figure 3.6 Failed Tension Test Specimens........................................ 34

Figure 3.7 Sensor Layout Screen.....................................................

Figure 3.8 2-D Location Setup Screen................................................ 35

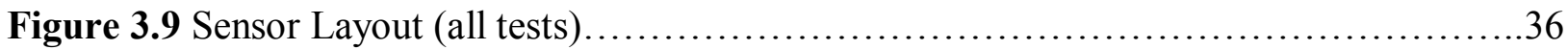

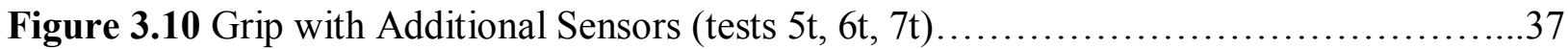

Figure 3.11 Hardware Setup Screen............................................. 38

Figure 3.12 Advanced Hardware Setup Screen...................................... 38

Figure 3.13 Test 1t Frequency vs. Amplitude.............................................39

Figure 3.14 Test 1t Amplitude vs. Duration............................................41

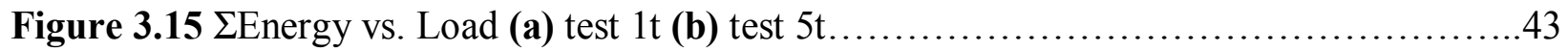

Figure 3.16 Test $7 \mathrm{t}$ Frequency vs. Amplitude $4^{\text {th }}$ quarter (a) ch. $1 \& 2$ (b) ch. $3 \& 4 \ldots \ldots \ldots \ldots . . . .44$

Figure 3.17 Test $7 \mathrm{t}$ Amplitude vs. Duration $4^{\text {th }}$ quarter (a) ch. $1 \& 2$ (b) ch. $3 \& 4 \ldots \ldots \ldots \ldots . . . .45$

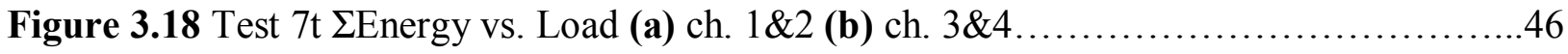

Figure 3.19 Test 4t Hits vs. Position vs. Load Unfiltered..................................47

Figure 3.20 Example of Sum of First Sensor Hit Plot Construction........................48

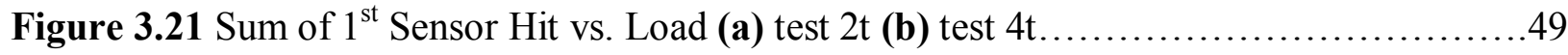

Figure 4.1 Unbroken Bending Specimens............................................59

Figure 4.2 INSTRON Loading Frame, Actuator, Controller, and Hydraulic Power Supply.....60

Figure 4.3 LVDT Calibration Tool.................................................60

Figure 4.4 LVDT Calibration Curve...............................................61

Figure 4.5 Specimen and Sensor Layout..........................................62

Figure 4.6 Unbent Specimen Prepared to be Loaded (B10)...........................63 


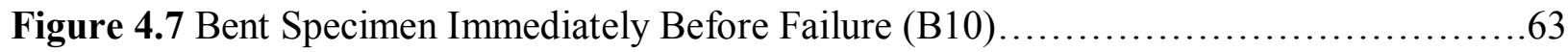

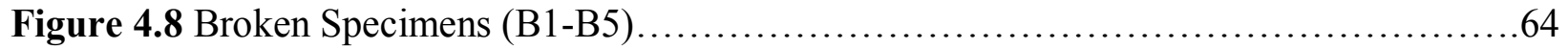

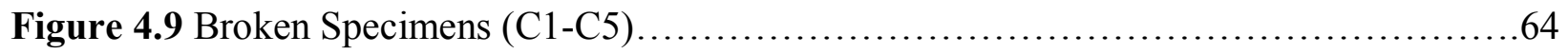

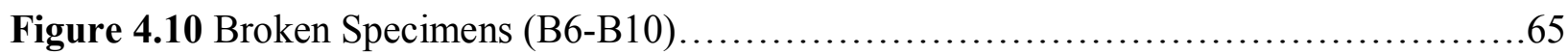

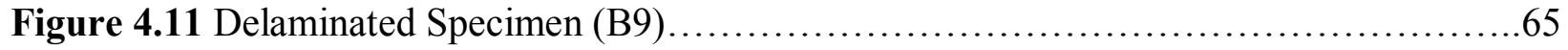

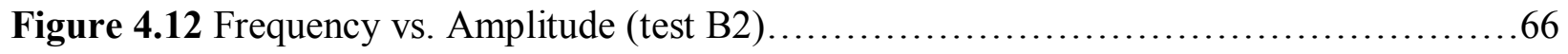

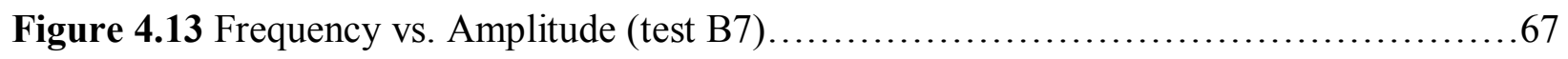

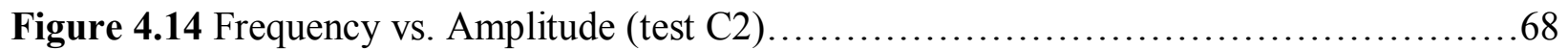

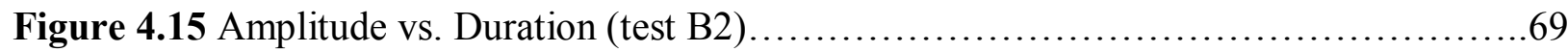

Figure 4.16 Amplitude vs. Duration (test B7) .................................... 70

Figure 4.17 Amplitude vs. Duration (test C2) ...................................... 71

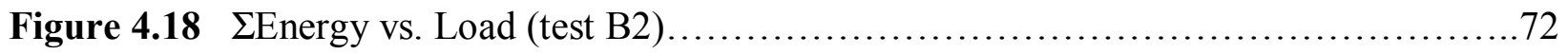

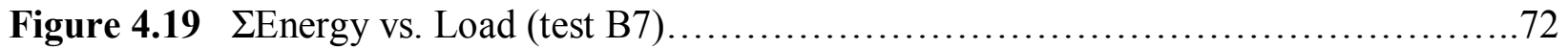

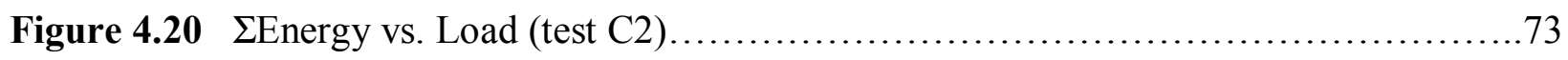

Figure 4.21 Loading Setup and Deflection Measurement Schematic.......................74

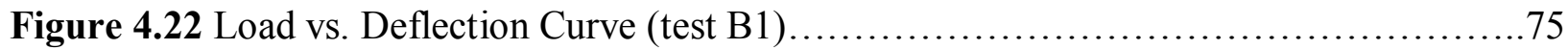

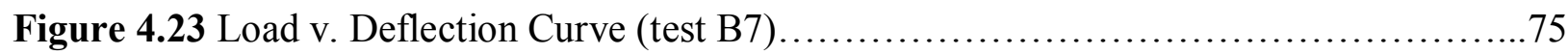

Figure 4.24 Load vs. Deflection Curve (test C4) .................................. 76

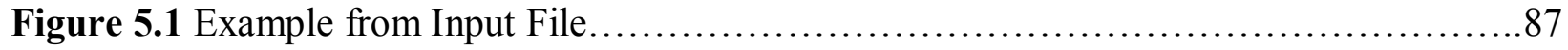

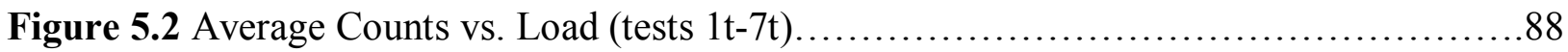

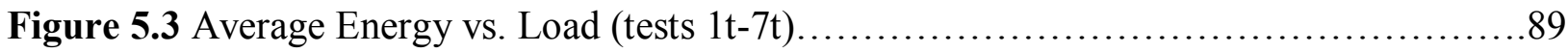

Figure 5.4 Average Duration vs. Load (tests 1t-7t) ................................. 90

Figure 5.5 Average Amplitude vs. Load (tests 1t-7t) ..............................91

Figure 5.6 Area of Interest on Frequency vs. Amplitude Plots............................92

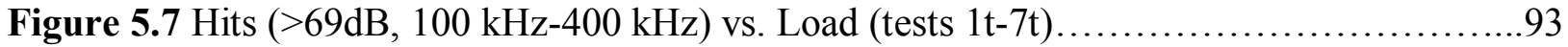

Figure 5.8 NN Architecture for Load Quarter Predictor................................94

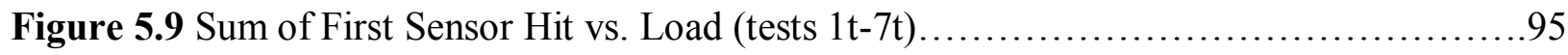

Figure 5.10 NN Architecture for Damage Zone Predictor...............................96

Figure 6.1 Average Counts vs. Load Parameter, Tests B1-B5..........................109

Figure 6.2 Average Counts vs. Load Parameter, Tests B6-B10.........................110

Figure 6.3 Average Counts vs. Load Parameter, Tests C1-C5 .........................111 
Figure 6.4 Average Energy vs. Load Parameter, Tests B1-B5 ..........................112

Figure 6.5 Average Energy vs. Load Parameter, Tests B6-B10.......................113

Figure 6.6 Average Energy vs. Load Parameter, Tests C1-C5 .........................114

Figure 6.7 Average Duration vs. Load Parameter, Tests B1-B5.........................115

Figure 6.8 Average Duration vs. Load Parameter, Tests B6-B10.........................116

Figure 6.9 Average Duration vs. Load Parameter, Tests C1-C5......................117

Figure 6.10 Average Amplitude vs. Load Parameter, Tests B1-B5.......................118

Figure 6.11 Average Amplitude vs. Load Parameter, Tests B6-B10......................119

Figure 6.12 Average Amplitude vs. Load Parameter, Tests C1-C5.......................120

Figure 6.13 Frequency Hits vs. Load Parameter, Tests B1-B5 .........................121

Figure 6.14 Frequency Hits vs. Load Parameter, Tests B6-B10.........................122

Figure 6.15 Frequency Hits vs. Load Parameter, Tests C1-C5 .......................... 123

Figure 6.16 Sum of First Sensor Hit vs. Load, Tests B1-B5 ........................... 124

Figure 6.17 Sum of First Sensor Hit vs. Load, Tests C1-C5 ........................... 125

Figure 7.1 Possible Technique for Damage Zone Predictor Using Sensors in a

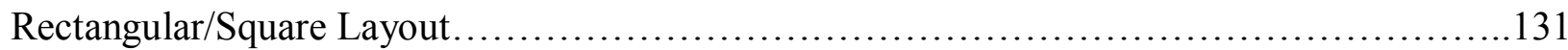




\section{Chapter 1}

\section{Introduction}

\subsection{Introduction}

Fiber Reinforced Polymer (FRP) composite bridge deck materials are relatively new to the civil construction market in West Virginia (WVU-CFC 2001). These materials have many advantages over traditional deck materials. One of these is corrosion resistance. Others are high strength and low self-weight. There are also situations inhibiting wider use of these materials. One such situation is the monitoring of the structural integrity.

Due to their complex structure, FRP materials cause the use of many types of nondestructive testing to be invalid. Among the complexities of the material is the many ways in which it can fail. These include matrix cracking, de-bonding/delamination, and fiber breakage failure. One such technique that can evaluate complex materials is acoustic emission (AE) testing (McIntire 1987). When the structure de-bonds, fibers break, and a release of elastic energy occurs. This release is termed acoustic emission. This energy propagates through the material in the form of stress waves. Piezoelectric sensors can then be used to detect these stress waves. Structures such as FRP pressure vessels have demonstrated that AE is a viable method for determining the integrity of FRP composite structures (ASTM E 1067).

Before a technique (AE included) can be used to evaluate a structure, laboratory tests to determine the technique's viability must be done. By performing laboratory tests, these $\mathrm{AE}$ parameters can then be connected to the status of the structure. For example, the AE parameters can be used to determine the loading that the structure is experiencing. In the end, these connections can be used along with computer analysis to create a health monitoring system for 
the structure. One such computer analysis tool that can be used to help create such a system is Neural Network analysis.

Neural Network (NN) analysis uses a complex set of equations and weighting functions to connect data to results. After the NN analysis has found these weights, they can be used to predict unknown results for other structures. This allows for a computer to analyze a data set and reach a conclusion without the aid of an operator which can decrease the chance of human error. Neural network programs have shown promise in other applications such as the recognition of faces (Mitchell 1997).

\subsection{Objectives and Scope}

The objective of this study is to take the first step in creating a user-friendly health monitoring system for FRP bridge deck structures using acoustic emissions and neural network analysis. This is to be done using AE parameters such as energy, duration, and amplitude to make connections to structural status such as load and damage zone. AE data are often very immense and scattered. Because of this, the data required some degree of filtration and concentration to achieve connections to structural status. These connections are then transformed into proper form for use with the neural network system.

To begin this project, tension tests on hand-laid specimens were first conducted to understand the material. ASTM D 3039 was used as a guide for the tension tests. These tension specimens were created in-house at West Virginia University. Relationships between the AE parameters of these specimens and load, along with damage zone were achieved. These were then used to conduct NN analysis on the tested specimens. Following the tension tests, bending tests were conducted to begin simulating the conditions of a bridge deck sections. These bending 
tests were conducted on three sets of five specimens from different manufacturers. In order to proportion these specimens, ASTM D 790 was used as a guide for their preparation. These sets included a set that was cut from a Creative Pultrusions Superdeck section, a set from Bedford Reinforced Plastics Inc. bridge deck section, and a set from a Bedford Reinforced Plastics Inc. plate section with different architecture than the deck section. These bending tests were conducted as four-point bending tests and were loaded to failure. The data was analyzed as with the tension tests, and used with the NN system.

\subsection{Arrangement of Contents}

Following this introduction (Chapter 1) is a literature review pertaining to Acoustic Emissions, FRP composite materials, and Neural Networks. This information is contained in Chapter 2, which is followed by a description of the tension tests along with their results in Chapter 3. The procedures and results for the bending tests of FRP specimens are contained in Chapter 4. This is followed by Chapters $5 \& 6$, which contain information on Neural Network analysis for the tension and bending specimens, respectively. Conclusions, applications, and recommendations formulated from this study are described in Chapter 7. All graphs for tension and bending tests that were not used in the body of the text are presented in the appendix section. 


\section{Chapter 2}

\section{Literature Review}

\subsection{FRP Composites}

\subsubsection{History and Background}

Since the 1960's, FRP materials have been the material of choice for the Aerospace industry. Only recently have glass FRP materials begun to garnish attention from the Civil engineering community for the bridge construction industry (WVU-CFC 2001). As these materials become better understood, various usages will begin to develop.

FRP composites consist mainly of two materials, the fiber and the resin. Materials used for the fiber component include glass, carbon, and aramid, with glass being the most common among these. One common configuration of these glass fibers are a roving. A roving consists of a number of fiber strands collected into a parallel bundle without any twisting. These rovings can then be used to create mats, woven fabrics, braids, knitted fabrics, and hybrid fabrics. These mats or fabrics of reinforcements are then combined with the polymer-resin matrix. By combining these materials, a new composite material that is much more useful than its components is created. The polymer resins fall into two groups, thermosets and thermoplastics. During curing, thermosets cross-link and become set in a final form. Thermoplastics on the other hand have molecular chains that are processed at higher temperatures and remain plastic. This allows for them to be reheated and reformed. For civil applications, thermosets are more commonly used. Some examples of thermoset resins are; polyesters, vinylesters, phenolics, and urethanes. Some methods of production of these composite materials are; hand lay up, pultrusion, resin transfer molding (RTM), injection molding, and compression molding (Tong et al. 2002). 
The nature of FRP composites, like other composites, results in failure happening in various ways. For FRP composites these are matrix cracking, matrix-fiber debonding/delaminating, and fiber breakage. Matrix cracking is when the polymer matrix begins to crack. When the polymer-resin matrix begins to separate from the fiber reinforcement, it is said to be debonding or delaminating. Fiber breakage is exactly as it sounds; the fibers begin to break.

\subsubsection{Advantages/Disadvantages}

Some of the advantages and disadvantages of these materials are listed below.

\section{$\underline{\text { Advantages }}$}

1.) corrosion resistance

2.) stiffness-to-weight ratio and high strength

3.) higher energy absorption

4.) low self-weight (in deck situations)

5.) competitive (when lifetime and maintenance cost is accounted for)

6.) can be molded into specific shapes

\section{Disadvantages}

1.) high initial cost

2.) lack of understanding of material

3.) lack of material and design specifications

4.) availability 


\subsubsection{Usage}

Usage of FRP composite materials in the civil community has recently been increasing as a greater understanding of these materials has developed. Among these uses, is FRP reinforcing wrap for damaged columns or beams (WVU-CFC 2001). FRP reinforcing bars are another usage of this material. These are especially prevalent in hospital structures, where much sensitive equipment is present. Research is also being done pertaining to using these bars as reinforcement in bridge decks and concrete pavements (Choi \& Chen 2003).

Another important usage of these FRP composite materials is in bridge decks. Use of FRP bridge deck materials for new and rehabilitated structures is growing. One particular advantage, FRP materials light weight, often allows for weight limits on bridges to be removed once they are rehabilitated using FRP deck materials. Some of the states that have instituted bridge decks using these materials are Delaware (Soneji et al. 2002), New York (O'Connor 2002), Pennsylvania (Werts 2002), Maryland (Robert 2002), and West Virginia (WVU-CFC 2001).

\subsection{Acoustic Emissions}

\subsubsection{History and Background}

It is unknown when the first acoustic emissions were noticed, but it is thought that fractures such as twigs breaking, rocks cracking, and bones breaking were among the first. One of the first usages, by artisans, may have been in the pottery making process. By relying on the audible cracking sounds of the clay articles that were cooling in the kiln, the potters could predict the quality of their work. It is often assumed that tin cry was one of the first observed cases of 
acoustic emission in metals. Because of this, much of the early research into acoustic emissions centered on tin with other metals following thereafter.

Transient elastic stress waves are created by the very rapid release of energy from localized sources. This is termed acoustic emissions. When most materials used in civil structures are cracked or deformed, they emit this acoustic energy. Because of this need for an introduced stress, acoustic emission is considered a passive method of nondestructive testing (NDT). This also means that unlike other methods of NDT, external energy does not need to be introduced into the $\mathrm{AE}$ monitoring system. Acoustic emissions emitted from sources throughout the structure, propagate through that structure and are detected by acoustic emission sensors. These sensors collect the data, which is then amplified and filtered to eliminate extra noise. These data are then collected and the pertinent parameters are saved and analyzed.

The use of acoustic emissions monitoring has expanded into numerous materials. Wood is one of these materials. One of the first uses of $\mathrm{AE}$ for wood most likely began in the $19^{\text {th }}$ century. In that time period, the creaking of mineshaft timbers was used to warn of a possible mine collapse. More recently, acoustic emissions have been used to characterize hardwoods from West Virginia (Chen et al. 1992). Acoustic emissions have also been used for purposes such as detecting the debonding of reinforcing bars in concrete (Hawkins et al. 1988).

The first use of acoustic emissions in FRP composite materials was the testing of fiber reinforced pressure vessels. The Society of the Plastics Industry (SPI) developed the procedure for glass reinforced vessels. The American Society for Testing Materials (ASTM) and the American Society of Mechanical Engineers (ASME) have also adopted similar procedures (McIntire 1987). 


\subsubsection{Important Terms}

Some of the important terms for AE parameters are defined below (McIntire 1987).

Acoustic emission count-The number of times the signal amplitude exceeds the preset threshold.

Acoustic emission event-A microstructural displacement that produces elastic waves in material under load or stress.

Couplant-A substance providing an acoustic link between the propagation medium and the transducer.

Reference threshold-A preset voltage level that has to be exceeded before an acoustic emission signal is detected and processed. This threshold may be adjustable, fixed, or floating.

Source-The place where an event takes place.

\subsubsection{Acoustic Emissions in FRP Materials.}

One important phenomenon displayed by acoustic emissions is known as the Kaiser effect. This was named for an early German acoustic emission researcher, Josef Kaiser, who did much pioneering work in the 1950's. He is also often credited with being the founder of modern acoustic emission technology. The Kaiser effect is the phenomenon where a structure will only produce $\mathrm{AE}$ signals when loaded to a higher level than previous loads. For example, if a structure was loaded to $100 \mathrm{lbs}$ and unloaded, for the next loading AE signals would only be detected at load levels higher than 100 lbs. In some materials, particularly FRP materials, there is a breakdown of the Kaiser effect. In these materials, signals can be detected at all loading levels, even when the structure was previously loaded. This is known as the felicity effect. 
One difficulty in AE analysis of FRP materials is locating the failure. This is very difficult because of the many types of failure in FRP composites (matrix cracking, debonding, and fiber breakage). The nature of this material causes it to have many cracks or small failures spread throughout the structure. Eventually these will concentrate and the specimen will fail, but it results in difficulty predicting final location.

Beyond FRP pressure vessels, the use of AE in FRP structures is limited. Acoustic emissions have been used to identify damage in stressed Aramid FRP bars (Chen et al. 1993). $\mathrm{AE}$ has also been used to identify fatigue failure modes in carbon fiber reinforced composites (Wevers et al. 1991). AE along with acoustic waveguides has also been used to monitor fiberreinforced plastic structures (Chen et al. 1994) and the cure and structural integrity of composite materials (Harrold \& Sanjana 1986).

\subsubsection{Wave Propagation}

Acoustic emission events are created from each sensor's response to stress waves generated in the material to which it is attached. Because of this, the principles of wave propagation are important to acoustic emission study. Two types of waves are present when a medium has no boundaries. In this infinite medium, the first of these waves are the dilatational or P waves. The other waves are the distortional or equivoluminal waves, which are also known as $\mathrm{S}$ waves. The wavespeed for these waves are given by the following equations.

$$
\begin{gathered}
c_{1}=\sqrt{\frac{\lambda+2 \mu}{\rho}} \text { (dilatational wave) } \\
c_{2}=\sqrt{\frac{\mu}{\rho}} \text { (distortional wave) }
\end{gathered}
$$

where, 
$\rho=$ mass density of the medium

The material parameters $\lambda$ and $\mu$, also known as Lame's constants, are found from the following equations.

$$
\begin{gathered}
\lambda=\frac{E v}{(1+v)(1-2 v)} \\
\mu=\frac{E}{2(1+v)}
\end{gathered}
$$

Where,

$$
\begin{aligned}
& E=\text { Young's modulus of the material. } \\
& v=\text { Poisson's ratio of the material. }
\end{aligned}
$$

In the case when a free surface is present in a medium, another type of wave exists besides the previous two. This third type of wave is termed a surface or Rayleigh wave. Although these three types of waves are generally independent and have different velocities in a semi-infinite solid with a free surface, coupling does occur in solids that have intersecting surfaces or in surfaces that have boundary conditions that are different throughout the surface. Wave propagation speed becomes particularly important when calculating signal locations. Generally surface waves are used for this purpose, and the accuracy of determination of their speeds has a great effect on the location accuracy.

\subsubsection{Acoustic Emission Waveform Parameters}

In order to understand and classify the AE signals received, a general understanding of the waveform parameters is needed. Some of the more common parameters are; rise time, counts, energy, duration, and peak amplitude. The peak amplitude is the highest voltage 
(amplitude) achieved during a particular AE signal. Before understanding the counts parameter, the threshold amplitude must be described. The threshold amplitude is the minimum voltage (amplitude) signal that the system will allow to be recorded. This allows for much of the background noise to be eliminated with just an adjustment of the threshold amplitude. The counts parameter is the number of times the signal amplitude crosses the threshold amplitude. The energy recorded is the area between the threshold amplitude and the line connecting the peak amplitudes. This quantity is a representation of the true energy released during AE activity. Each signal has a peak amplitude, which is simply the highest amplitude signal from a certain event. The time between when the first signal crosses the threshold and when the last signal crosses the threshold is termed the duration. The final parameter, rise time, is the time between the first threshold crossing and when the peak amplitude is achieved. All of these parameters can be seen labeled on the typical waveform shown in figure 2.1 .

\subsection{Neural Networks}

\subsubsection{Background and History}

Neural networks (NN), or artificial neural networks (ANN) as they are sometimes called, are a type of analysis that is roughly modeled after the structure of the brain. The early history of neural networks can be traced as far back as the late 1800's. At that time, James, an American psychologist, was the first person to publish a number of facts related to the brain's structure and function. Among these, were some of the basic principles of correlational learning and associative memory (Eberhart et al. 1996). Since the late 1980's, with the aid of increasingly more powerful personal computers, development of neural networks has expanded greatly. 


\subsubsection{General Principles}

Neural networks can be considered an information processing system. By accepting input data, the neural network can then map them into output data. Initially, input data and output data are both input together in what is considered the training set. The neural networks then make numerical connections between the input data and output data. These hidden weights can then be recalled and used to predict unknown results for a given input test set. A schematic of a typical Neural Network architecture can be seen in Figure 2.2. This type of computer learning is particularly effective for training data from noisy, complex sensor data; for example, data from cameras or microphones.

When training a neural network system, it is important to have a measure of performance of the prediction. Two such parameters used are correct rate (CR) and root mean square (RMS). When either parameter reaches its set value, the training process has converged. The equations for each of these are as follows.

$$
\begin{gathered}
C R=\frac{(\text { number of patterns correctlyclassified })}{(\text { total number of patterns of the data sets })} \times 100 \% \\
R M S=\frac{1}{P} \sum_{i=1}^{P}\left[\frac{1}{m} \sum_{j=1}^{m} \sqrt{\left(d_{j}^{(i)}-y_{j}^{(i)}\right)^{2}}\right]
\end{gathered}
$$

where,

$$
\begin{aligned}
& \mathrm{P}=\text { total number of training patterns } \\
& \mathrm{m}=\text { the number of output units } \\
& \mathrm{d}_{\mathrm{j}}=\text { the predicted output } \\
& \mathrm{y}_{\mathrm{j}}=\text { the actual output }
\end{aligned}
$$




\subsubsection{Current Uses}

Current uses for neural networks are increasing. One of these is the use of neural networks to power face recognition software (Mitchell 1997). These face recognition systems have even been used at special events such as the Super Bowl to identify criminals. Neural Networks have also proven to be a useful tool in locating AE signals using acoustic waveguides (Chen \& Wissawapaisal 2000) and for processing signals from hardwoods with various treatments (Chen \& Chen 1992).

\subsection{Standards for AE Testing Techniques and FRP Composites}

During this study, guidelines were needed to complete the tests in a reliable manner. One useful source for such standards is ASTM. Some of the following standards were used in this study, while others are related to the topics covered in this study. Some of the ASTM standards for Acoustic Emission testing are as follows:

E 543: Standard Practice for Agencies Performing Nondestructive Testing

E 569: Standard Practice for Acoustic Emission Monitoring of Structures During Controlled Stimulation

E 650: Standard Guide for Mounting Piezoelectric Acoustic Emission Sensors

E 750: Standard Practice for Characterizing Acoustic Emission Instrumentation

E 976: Standard Guide for Determining the Reproducibility of Acoustic Emission Sensor Response

E 1067: Standard Practice for Acoustic Emission Examination of Fiberglass Reinforced Plastic Resin (FRP) Tanks/Vessels

E 1106: Standard Method for Primary Calibration of Acoustic Emission Sensors 
E 1118: Standard Practice for Acoustic Emission Examination of Reinforced Thermosetting Resin Pipe (RTRP)

E 1211: Standard Practice for Leak Detection and Location Using Surface-Mounted Acoustic Emission Sensors

E 1316: Standard Terminology for Nondestructive Examinations

F 914: Standard Test Method for Acoustic Emission for Insulated Aerial Personnel Devices

The following are the ASTM standards relating to FRP materials and the testing methods used during this study:

D 638: Standard Test Method for Tensile Properties of Plastics

D 790: Standard Test Methods for Flexural Properties of Unreinforced and Reinforced Plastics and Electrical Insulating Materials.

D 883: Standard Terminology Relating to Plastics

D 3039: Standard Test Method for Tensile Properties of Polymer Matrix Composite Materials

E 4: Standard Practices for Force Verification of Testing Machines 


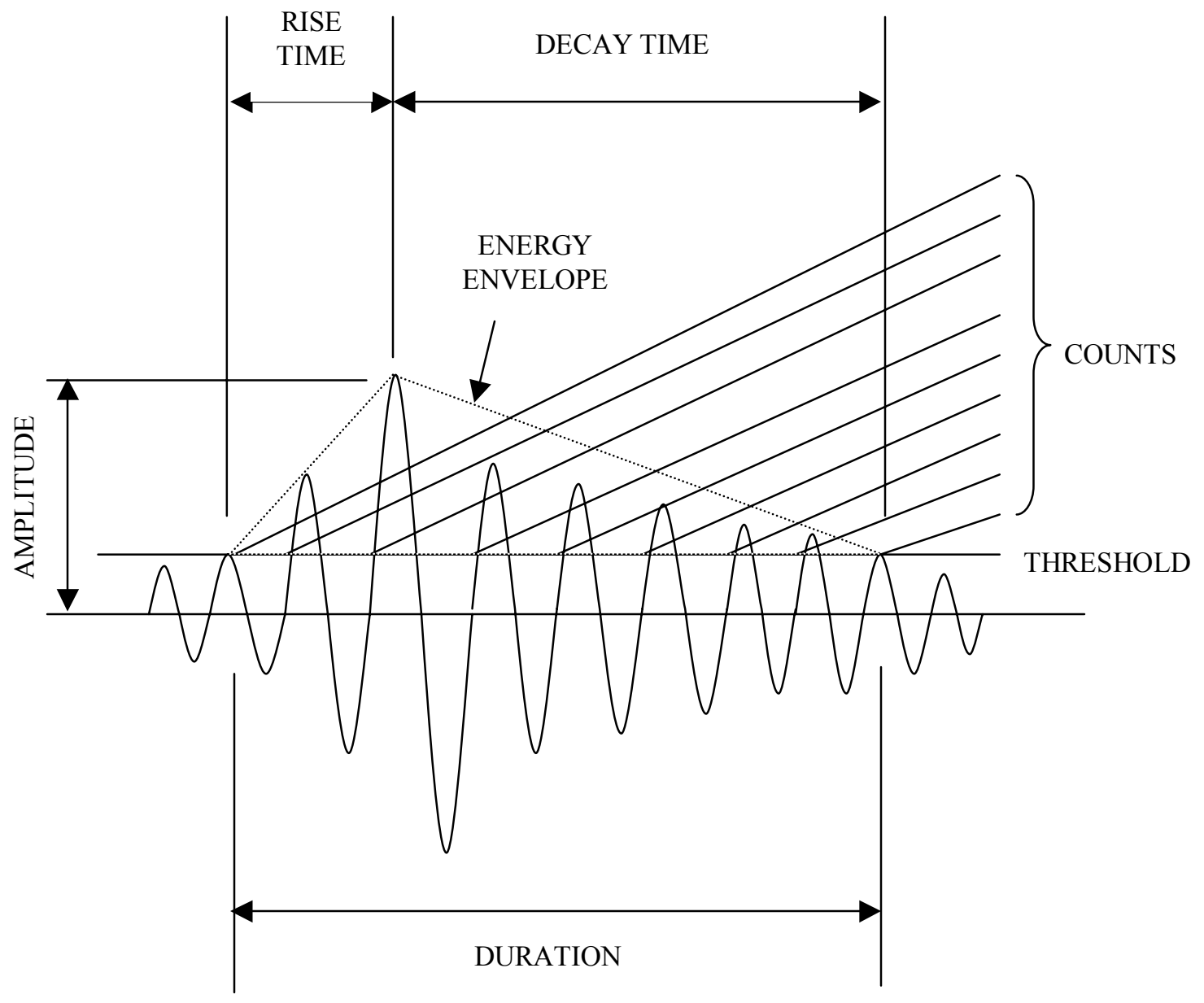

Figure 2.1 Typical AE Waveform and Parameters 

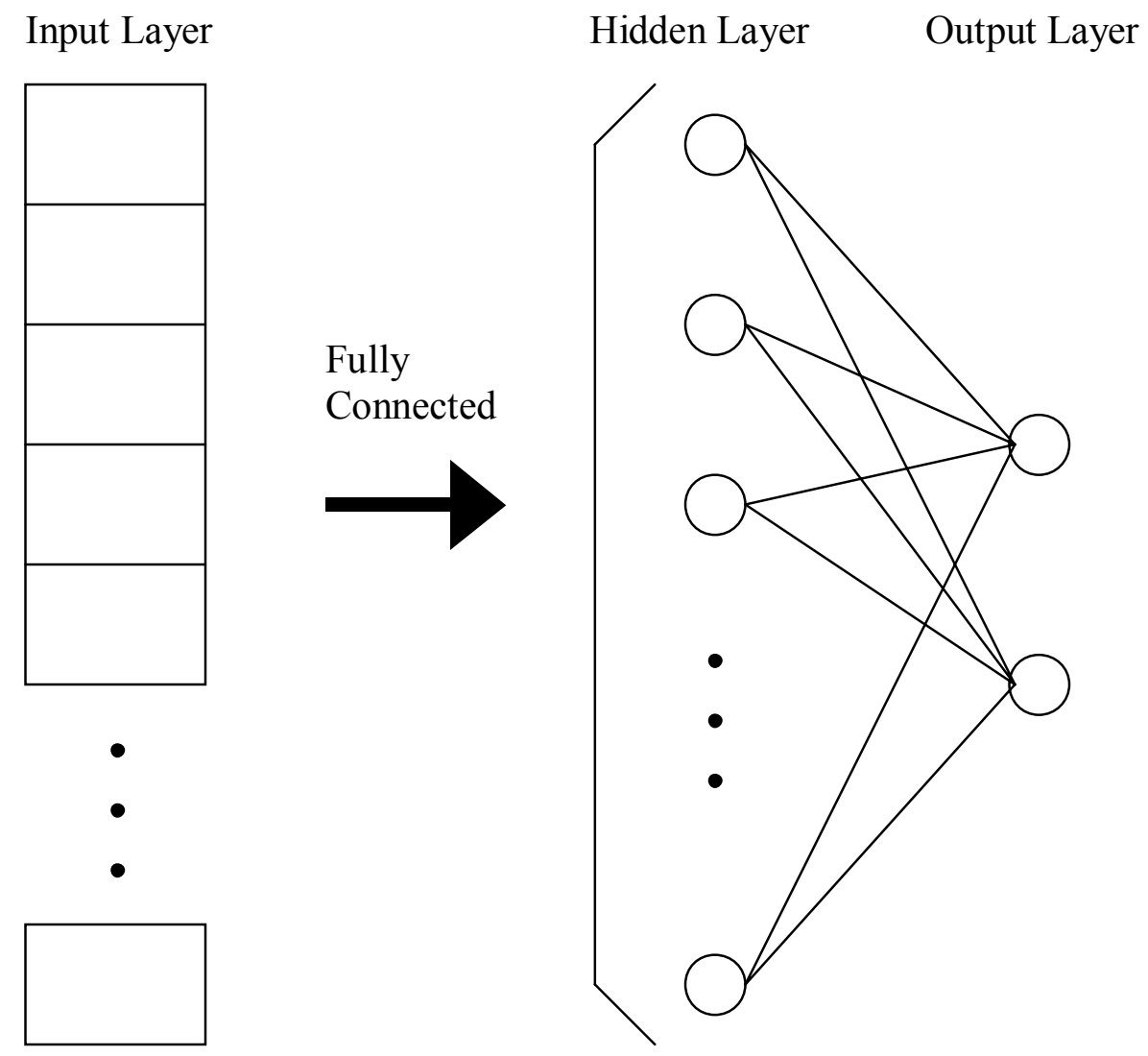

Figure 2.2 Typical Neural Network Architecture 


\section{Chapter 3 \\ Tension Tests}

\subsection{Specimens}

In order to get the acoustic emission (AE) characteristics of Fiber Reinforced Polymer (FRP) members, preliminary tension tests were conducted in accordance with ASTM D 3039/D 3039M-00. The specimens for these tests were produced in house at West Virginia University's Chemical Engineering Laboratory using the hand-laid method for producing composite materials (Yeh \& Cho 2003). A total of seven tension specimens were tested and are designated as $1 \mathrm{t}$ through 7t. The first specimen (1t) was a six-layer specimen, with each of the following six specimens (2t-7t) being composed of four fiber layers. Each of these specimens were Glass Fiber Reinforced Polymer (GFRP) composites. Durakane 411-350, produced by Dow Chemicals, was the epoxy vinyl ester resin used for these tension specimens. All specimens were of the same size, 7 inches long by 1 inch wide by .1 inch thick. Tabs for gripping were adhered to each end of each specimen.

\subsection{Instruments and Equipment}

\subsubsection{INSTRON Loading Frame}

Tension tests were conducted using an Instron 8501 loading frame controlled by a computer control panel, which in turn was powered by a hydraulic power supply source. A load cell was connected to the loading frame in order to record the load being applied. This load cell was connected to the LOCAN AT computer data acquisition system. A picture of the Instron 8501 along with the load cell and actuator can be seen in Figure 3.1. The hydraulic power supply is pictured in Figure 3.2. 


\subsubsection{Computer Controller}

An Instron model 8500 Plus control panel was used to control the Instron loading frame. This panel allows input for 2 strain readings, a load reading and a position reading. It also has a waveform generator. The panel is also connected to a PC. This allows for the loading to be controlled by load/time or displacement/time. It also allows for graphs to be displayed at realtime speeds. The complete Instron 8500 Plus control panel and PC setup can be seen in Figure 3.3.

\subsubsection{AE Equipment and Software Setup}

The AE acquisition system used for these tension tests was a LOCAN AT system (Physical Acoustics Corporation). The LOCAN AT system is updated to operate with SPARTAN 2000 software and is operated through a personal computer. This complete setup is seen in Figure 3.4. The LOCAN AT system has six channels for sensor inputs and four parametric inputs (three single-ended and one differential).

Piezoelectric (PICO) sensors with a diameter of .188in and a height of .157in were used. The PICO sensors, pictured in Figure 3.5(a), were each connected to the LOCAN AT data acquisition system through model 1220A preamplifiers. An example of the model 1220A preamplifier is seen in Figure 3.5(b). Note the toggle switch on the right side of the preamplifier allowing for the selection of a $40 \mathrm{~dB}$ or $60 \mathrm{~dB}$ gain. For our application, a gain of $40 \mathrm{~dB}$ was selected. 
The LOCAN AT system was also connected to the Instron computer controller in order to record the load being applied. The loading was collected through the first parametric input and is measured in voltage. The following equation is used to convert voltage to loading.

$$
\text { Displayed Value }=(\text { Voltage } * \text { Multiplier })+\text { Offset }
$$

To scale this voltage output, the correct Multiplier and Offset numbers were calculated using the following equations.

$$
\begin{gathered}
\text { Multiplier }=(\mathrm{L} 1-\mathrm{L} 2) /(\mathrm{V} 1-\mathrm{V} 2) \\
\text { Offset }=(\mathrm{V} 1 * \mathrm{~L} 2-\mathrm{V} 2 * \mathrm{~L} 1) /(\mathrm{V} 1-\mathrm{V} 2)=\mathrm{L} 1-\text { Multiplier } * \mathrm{~V} 1
\end{gathered}
$$

Where,

$$
\begin{aligned}
& \text { L1, L2 = Loading } \\
& \text { V1, V2 = Voltage }
\end{aligned}
$$

These values were found to be 2250 for multiplier and -30 for offset.

The SPARTAN 2000 software for the LOCAN AT system also allows for replaying tests at a later time. This allows for the changing of parameters on graphs, and location plots, and better analysis of data. In order to increase data recording speeds for all seven tension tests, no graphs were analyzed in real-time. All data was collected and replayed to obtain plots needed.

Two other programs of interest that are a part of the SPARTAN 2000 software set are ATPOST and ATASC. The ATPOST program allows for the filtering of recorded data. Data 
can be filtered using many parameters on an accept or reject basis. ATASC is simply for converting data files into text files.

\subsubsection{Location Software and Setup}

For the tension tests, 2-D location methods were used. In order to perform 2-D location, the Spartan SP2-LOC program was used. The sensor layout screen can be seen in Figure 3.7. On this page, parameters such as number of sensors and position of each sensor must be identified. Before the SP2-LOC program could locate signals, the wavespeed through the specimen was needed. To obtain the wavespeed, a common pencil break test was performed and the average speed was calculated. For the six-layer specimen (1t) this was found to be 141,600 in/seconds, and for the four-layer specimens 141,000 in/seconds. These calculations are shown in Tables 3.1 and 3.2 respectively. These wave speeds along with lockout time, over (ovecalculation time), and timing type were entered into the location setup screen. This screen can be seen in Figure 3.8. Lockout is the amount of time after an event is sensed that other signals are locked out. The minimum lockout time is the maximum distance between sensors. Over (overcalculation) is a parameter that allows for signal location even in the event that their arrival times are greater than the theoretical maximums. There are two choices for timing type, FTC and PT. FTC stands for first threshold crossing and PT stands for peak time. For these tests, FTC was chosen as the timing type. 


\subsection{Test Procedure}

\subsubsection{Sensor Layout}

On each of the first four tests, two sensors were placed on each specimen. The sensors were attached approximately two inches apart, with the center of the specimen being at the center of the sensor group. A schematic drawing of the specimens along with the location of sensors $1 \& 2$ (all tests) can be seen in Figure 3.9. For tests five and six, in addition to the two original sensors, two more sensors were attached to the Instron grip arm. This was in an attempt to capture any noise from the gripping of the specimen, and separate it from the true signals of the GFRP specimen. For the seventh and final tension test, the two additional sensors were attached to the wedge portion of the grip that is in direct connection with the specimen. The location of these additional sensors along with a schematic drawing of the tension testing machine's grip can be seen in Figure 3.10.

Another important consideration in our AE testing procedure was the bond between the AE sensors and the specimen. Not only do the sensors need to be strongly bonded to the specimen; they also need a couplant between themselves and the specimen. A couplant is a substance that provides an acoustic link between the specimen and the sensor. This couplant fills in the tiny, sometimes even microscopic gaps that exist between the two surfaces. A method of solid coupling was chosen. Solid coupling is achieved with glue or cement, which ensures continuity between the specimen and the sensor surface. A thin layer of hot melt glue was chosen for this study. It has been shown that a thin layer of hot melt glue sufficiently attaches sensors and acts as a couplant without resulting in large material attenuation (Fultineer 1997).

\subsubsection{Software Settings}


For each channel used, several settings must be entered. Among these were the gain and threshold. The gain + threshold value must fall within a range from $46 \mathrm{~dB}-88 \mathrm{~dB}$. A gain value of $20 \mathrm{~dB}$ is acceptable for medium to low sensitivity and $35 \mathrm{~dB}$ for high sensitivity. For our composite material a gain of $20 \mathrm{~dB}$ was selected. Along with magnitude, a type of threshold must be selected. For our application, a fixed threshold was selected. Before beginning the tests, a threshold value was established. The collection program ignores all signals occurring below the threshold value. By setting the threshold at the appropriate level, most background noise and constant noise created by the machinery can be eliminated. The best value for this threshold was found to be $45 \mathrm{~dB}$. This resulted in an acceptable gain + threshold value of $65 \mathrm{~dB}$. Each of these settings was entered into their respective slots in the standard hardware setup screen, which can be seen in Figure 3.11.

Peak Definition Time (PDT), Hit Definition Time (HDT), and Hit Lockout Time (HLT) are parameters that must be entered in the advanced channel setup section. A screen capture showing the advanced channel setup section along with input values can be seen in Figure 3.12. All three of these are timing parameters for the signal measurement process. PDT insures that the signal peak for risetime measurements is correctly identified. For general purpose testing, a value of 20-50 is recommended for composites. A mid-range value of 35 was selected for our application. The HDT parameter ensures that each AE signal is reported as one and only one hit. For Composite materials, a value of $100-200$ is recommended. As with the PDT value, a mid-range value was selected. That value was 150 . The recommended value of 300 was used for the HLT input.

\subsubsection{General Testing Procedures}

1. After adhering all sensors, the wave speed was found using a common pencil break test. 
2. The specimen was placed into the Instron testing machine.

3. All equipment connections were made. This included sensors to AE data acquisition system and load output to AE data acquisition system.

4. The Instron testing machine was zeroed, and the threshold was established (first test).

5. Loading of the specimen was initiated. The loading was controlled by displacement and the specimen was loaded to failure. Loading times varied from approximately 15 minutes up to about 45 minutes.

6. After the specimen failed, the AE data acquisition system was permitted to record the data. This data was saved on the system for later replays and analysis.

7. See Figure 3.6 for a picture of some of the broken specimens.

\subsection{Experimental Results}

Each specimen was loaded to failure. For the first six-layer specimen, this occurred at approximately 8 kips. For the final six four-layer specimens, failure occurred between 5 kips and 5.5 kips. In specimens $1 \mathrm{t} \& 4 \mathrm{t}$ the failure region was near the middle of the specimen (between the sensors). For all other tests, failure occurred outside of the sensors.

In order to have more control over graph parameters and settings, all recorded information was translated into text files using the ATASC program. This allowed for the data to be analyzed using Microsoft EXCEL spreadsheets. 


\subsubsection{Frequency vs. Amplitude}

One graph plotted to find trends indicating failure was Frequency vs. Amplitude. For each test, the loading cycle was split into four quarters, and Frequency vs. Amplitude graphs were plotted for each of these quarters. By splitting the loading into four sections, it was hoped that a trend warning of impending failure could be found. In the early loading stages, mainly the first two quarters, the signals are scattered throughout the frequency range but stay at lower amplitude values. This is seen in Figures 3.13 (a) and (b). In the later quarters of the loading, an interesting pattern begins to present itself. As the loading nears the failure load, high amplitude signals (70 dB-100 dB) congregated between $100 \mathrm{kHz}$ and $400 \mathrm{kHz}$ appear. Figures 3.13 (c) and (d) show this signal trend. These signals are thought to be emanating from fiber breakage. The appearance of these signals could warn of an increased probability of failure. Specimen $1 \mathrm{t}$ is used as a typical example. Results for the remainder of the specimens can be found in Appendix-A.

\subsubsection{Amplitude vs. Duration}

Another series of graphs plotted were Amplitude vs. Duration plots. As with the previous Frequency vs. Amplitude graphs, these graphs were plotted per quarter of loading cycle. A definitive trend was also observed in this series of graphs. In the early loading stages (Figures 3.14 (a) and (b)), the signals congregate in an area of low amplitude and low duration, but as the loading approaches the final quarter (Figure 3.14 (d)) signals of high amplitude and high duration begin to appear. These signals tend to indicate nearing failure. Appendix-B contains the plots for the remaining specimens. 


\subsubsection{EEnergy vs. Load}

For each of the seven tension specimens, summation Energy vs. Load graphs were plotted. $\Sigma$ Energy vs. Load graphs indicate the level of signal activity at certain loads. For each of the specimens, one trend held true; as the loading approached failure, the slope of the graph decreased. This would indicate that as the specimen nears failure, the signals, although stronger, are fewer in number. This change in slope occurs earlier and much more abruptly in the specimens that failed between the sensors. Figure 3.15(a) shows $\Sigma$ Energy vs. Load for such a specimen, specimen 1t. Test 4t, as seen in Appendix-C, also shows this same trend. This is caused by the fact that more signals with less attenuation will be captured from a failure that occurs between the sensors. Figure 3.15 (b) is the graph for test 5t. This specimen failed outside of the two sensors and shows the trend of a later more gradual slope change. Tests $2 \mathrm{t}, 3 \mathrm{t}, 5 \mathrm{t}, 6 \mathrm{t}$, and $7 \mathrm{t}$ all show this same pattern and can be seen in Appendix-C.

\subsubsection{Noise Elimination}

One important problem encountered with these tension tests was the elimination noise signals from the grips. The nature of the grips, cleated steel being clamped into the specimen and then pulled, would appear to create many signals. The first two attempts (tests 5t\&6t) at capturing grip noise proved to be unsuccessful. In these two tests, the many material transitions between the grip area and the sensors caused the signals to deteriorate greatly. On the third attempt (7t) to capture the grip noise, the sensors were placed on the wedge that directly grips the specimen. Because the wedge was in direct contact with the specimen, signal lose due to material transitions was minimized. 
The plots for sensors $1 \& 2$ show the same patterns as the previous six tension tests. In the last quarter of loading, the pattern of high amplitude signals between frequencies of $100 \mathrm{kHz}$ to $400 \mathrm{kHz}$ holds true. The plot of the $4^{\text {th }}$ quarter of loading for test $7 \mathrm{t}$ shows this in Figure 3.16 (a). For sensors $3 \& 4$, during the last loading quarter, signals between frequencies of $100 \mathrm{kHz}$ to $400 \mathrm{kHz}$ also appear. However, these signals occur at lower amplitudes (not crossing $85 \mathrm{~dB}$ ), as seen in Figure 3.16 (b). This indicates that the signals from the grips, while occurring at similar frequencies, appear at lower amplitudes. This validates the argument that high amplitude, high duration signals between frequencies of $100 \mathrm{kHz}$ to $400 \mathrm{kHz}$ are true signals from the specimen. Comparison of the Amplitude vs. Duration graphs provided no useful elimination of noise. As Figures 3.17 (a) and (b) show, all sensors produced large duration signals, with those from sensors $3 \& 4$ being of longer duration. Sensors $3 \& 4$ produced longer duration signals due to ringing of signals in the metal. This ringing is shown in Figure 3.17 (b). Comparison of the $\Sigma$ Energy vs. Load graphs provides more useful conclusions. For sensors $1 \& 2$, the same pattern of a steeper slope with a turning point followed by decreased slope holds true. For sensors $3 \& 4$ there is no turning point, and the slope increases as loading increases. If a large amount of noise signals from the grips was present, this plot would show a much larger increase in the beginning. This means that the majority of signals recorded by sensors $3 \& 4$ are also coming from the specimen and not the grips, and are attenuating before reaching these sensors. This attenuation causes many signals at the beginning (low amplitude) to disappear before reaching sensors $3 \& 4$. These graphs are shown in Figures 3.18 (a) and (b). Note that there are kinks on Figure 3.18. This was caused due to the loading being stopped, held, and then restarted. 


\subsubsection{Location Plots/Damage Zone}

Location plots were also developed using the Spartan software. One potential problem with the location determination is the fact that the material changes as more load is applied. For example, as the loading increases, more cracks and delaminations begin to appear. These flaws hinder the signals as they propagate through the specimen, causing slower wavespeeds and higher material attenuations. This causes the accuracy of the location plots to be compromised as the loading increases. As a result of the use of the linear location method, only signals between the two sensors were able to be located. All signals occurring outside of the sensor region are shown to have occurred directly at the sensor. Another drawback to the use of linear location is that only signals occurring between the two sensors can be located. This graph was split into two sections in order to more easily see the later portion of the loading. Figure 3.19 shows the location plot for test 4t. As you can see, the signals are spread throughout and generally show no trend toward indicating the location of final breakage.

Because of the difficulties of definitively locating the signals of the failure location in FRP materials, a new method of determining areas of interest had to be used. It was decided to use a method of first sensor hit. For all locatable signals (signals with $\Delta t$ less than the maximum found from wavespeed calculation) a value of 1 or 2 was assigned based on which sensor received the signal first. A schematic of this method can be seen in Figure 3.20. The summation of the number of times each sensor was hit first was plotted against the loading percent. This is called Sum of the First Sensor Hit plot. This plot gives an indication of the damage zone on the specimen. If the curves for both sensors have equal slopes, the specimen is being damaged in the middle. If not, the specimen is being damaged in the zone of the sensor that relates to the curve with the higher slope. The following equations were used to develop these curves: 


$$
\begin{gathered}
\Delta_{\text {time }}>\left\|t_{1}-t_{2}\right\| \\
\Delta_{\text {time }}=\frac{l}{v} \\
m=\frac{\left[(\text { sum })_{i}-(\text { sum })_{j}\right]}{2.5} \\
\Delta_{m}=m_{2}-m_{1}
\end{gathered}
$$

where,

$$
\begin{aligned}
& \Delta_{\text {time }}=\text { Maximum time difference between sensor hits for true signals. } \\
& \text { l= Distance between sensors. } \\
& v=\text { Wavespeed of specimen. } \\
& \mathrm{t}_{1}, \mathrm{t}_{2}=\text { Time when a signal hits the respective sensor. } \\
& \mathrm{m}=\text { Slope of Sum of First Sensor Hit curve. } \\
& \left.\left[(\text { sum })_{\mathrm{I}}-(\mathrm{sum})_{\mathrm{j}}\right)\right]=\text { Change in rise on Sum of First Sensor Hit curve. }
\end{aligned}
$$

note: the number 2.5 is used because the slope is being found for each load percent interval $(2.5 \%)$

$\Delta_{\mathrm{m}}=$ Slope difference between curves for sensors 1 and 2.

$\mathrm{m}_{1}, \mathrm{~m}_{2}=$ Slope of Sum of First Sensor Hit curve for respective sensor. 
Figures 3.21 (a) and (b) display this trend. In Figure 3.21(a), the plot for test 2t, the two lines run at the same slope early on indicating a damage zone in the middle. Plots for the remaining specimens can be seen in Chapter 4. Towards the end of the loading, the line for sensor two has a larger slope increase that indicates a damage zone near sensor two. This is the area where this specimen failed. Figure 3.21(b), the plot for test 4t, shows that the slopes of both lines remain nearly identical to one another. This indicates a damage zone in the middle, which is where the specimen failed. 


\begin{tabular}{|c|c|c|}
\hline \multicolumn{3}{|c|}{ Six-Layer } \\
\hline & & \\
\hline$\Delta \mathrm{t}$ & $\Delta \mathrm{d}$ & $\mathrm{v}$ \\
\hline$(\mu$ seconds $)$ & (inches) & $($ in/s $)$ \\
\hline 13 & 2 & 153846 \\
\hline 15 & 2 & 133333 \\
\hline 14 & 2 & 142857 \\
\hline 14.6 & 2 & 136986 \\
\hline 14.7 & 2 & 136054 \\
\hline 15.2 & 2 & 131579 \\
\hline 14 & 2 & 142857 \\
\hline 14.2 & 2 & 140845 \\
\hline 14 & 2 & 142857 \\
\hline 14.5 & 2 & 137931 \\
\hline 13.8 & 2 & 144928 \\
\hline 13.6 & 2 & 147059 \\
\hline 13.7 & 2 & 145985 \\
\hline 13.8 & 2 & 144928 \\
\hline \multicolumn{3}{|c|}{} \\
\cline { 2 - 3 } & AVERAGE & 141575 \\
\cline { 2 - 3 }
\end{tabular}

Table 3.1 Wave Speed Calculation of Six-Layer Specimen

\begin{tabular}{|c|c|c|}
\hline \multicolumn{3}{|c|}{ Four-Layer } \\
\hline$\Delta t$ & $\Delta d$ & $y$ \\
\hline ( $\mu$ seconds) & (inches) & $(\mathrm{in} / \mathrm{s})$ \\
\hline 14.2 & 2.125 & 149648 \\
\hline 16.5 & 2.125 & 128788 \\
\hline 15.3 & 2.125 & 138889 \\
\hline 16 & 2.125 & 132813 \\
\hline 16 & 2.125 & 132813 \\
\hline 14.2 & 2.125 & 149648 \\
\hline 16.2 & 2.125 & 131173 \\
\hline 14.2 & 2.125 & 149648 \\
\hline 14.3 & 2.125 & 148601 \\
\hline 16 & 2.125 & 132813 \\
\hline 14.3 & 2.125 & 148601 \\
\hline 14.4 & 2.125 & 147569 \\
\hline 16.2 & 2.125 & 131173 \\
\hline 14 & 2.125 & 151786 \\
\hline & $\overline{\mathrm{AVERAGI}}$ & 140997 \\
\hline
\end{tabular}

Table 3.2 Wave Speed Calculation of Four-Layer Specimen 


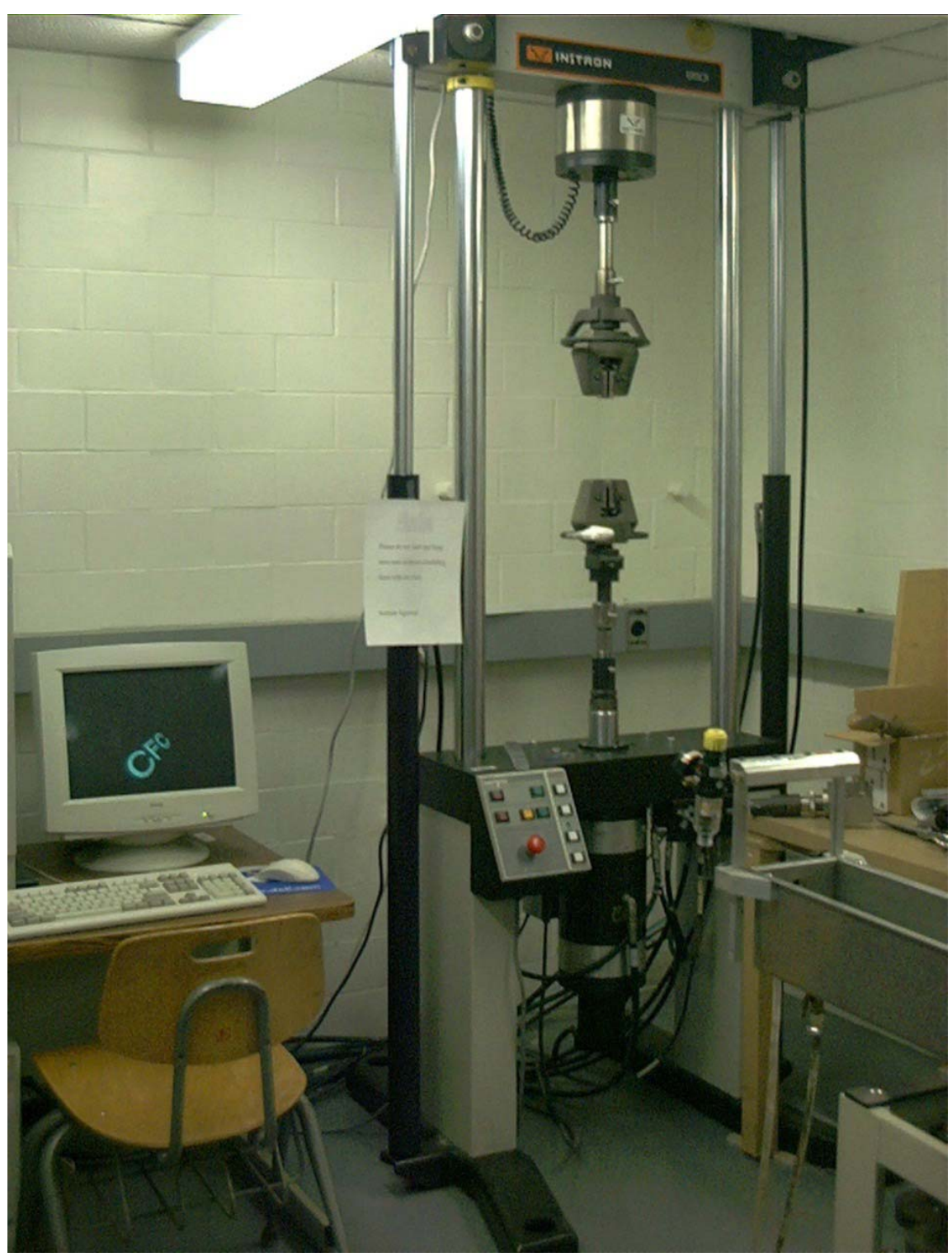

Figure 3.1 INSTRON 8501 Loading Frame, Load Cell, and Actuator 


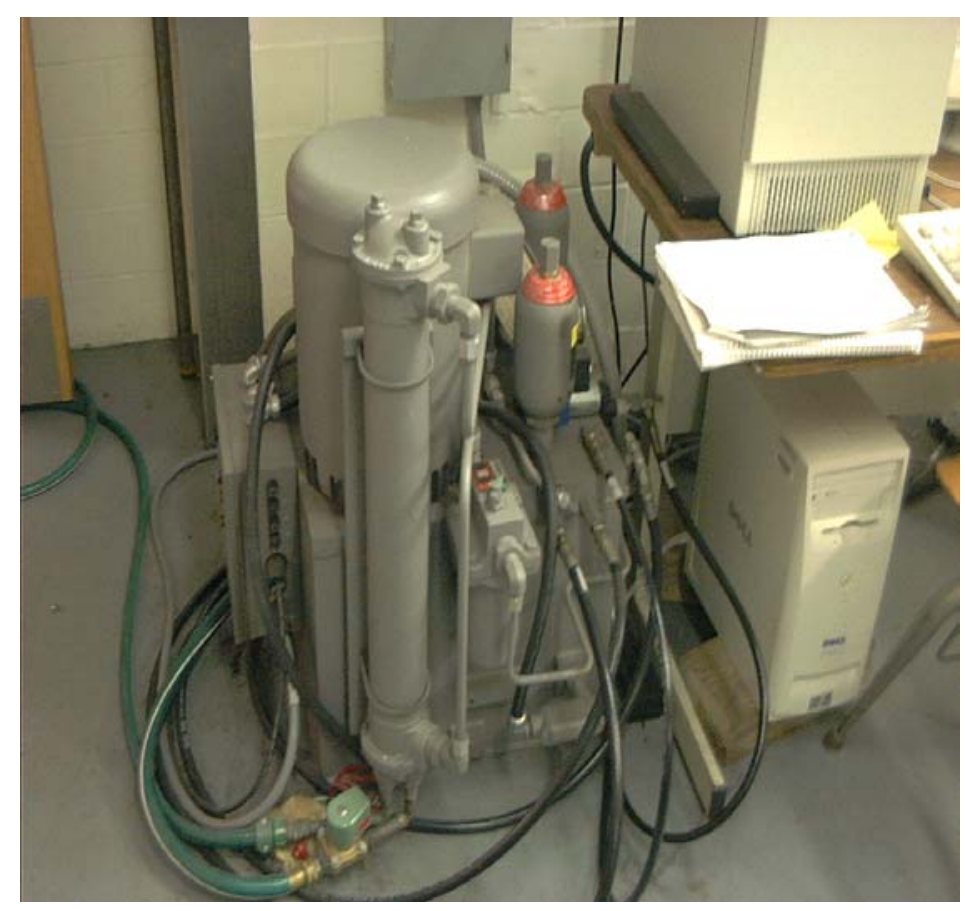

Figure 3.2 Hydraulic Power Supply

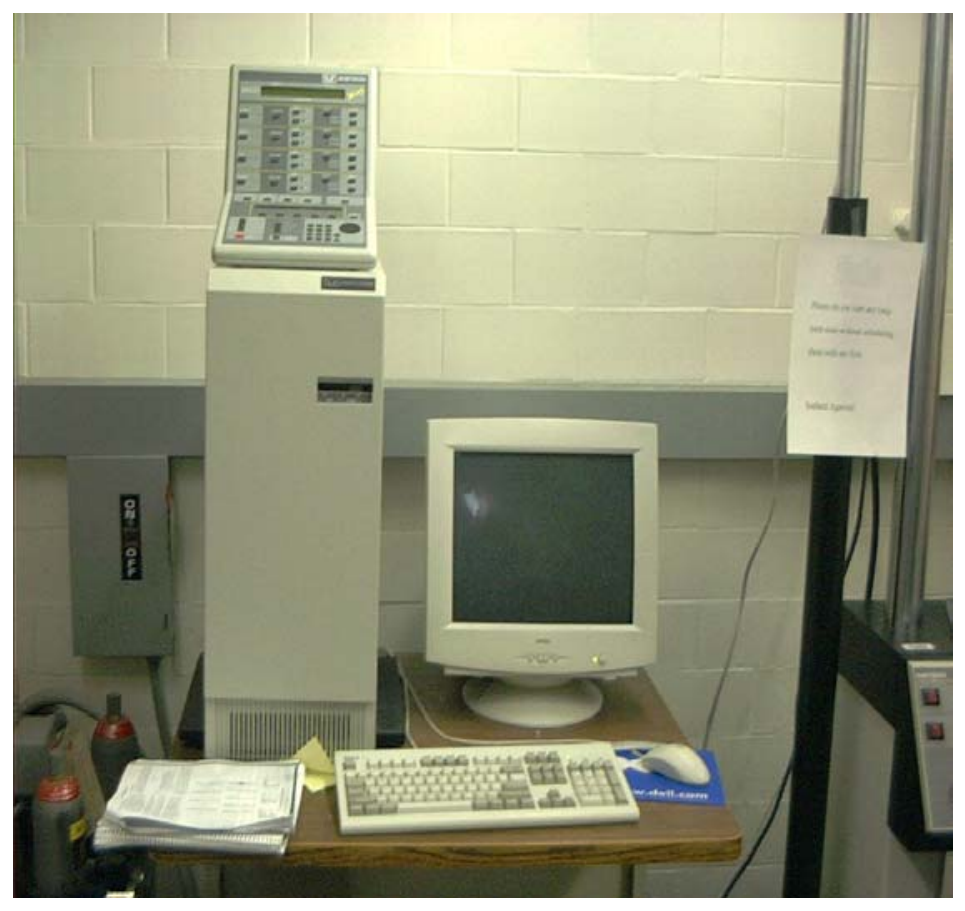

Figure 3.3 INSTRON 8500 Plus Control Panel and PC 


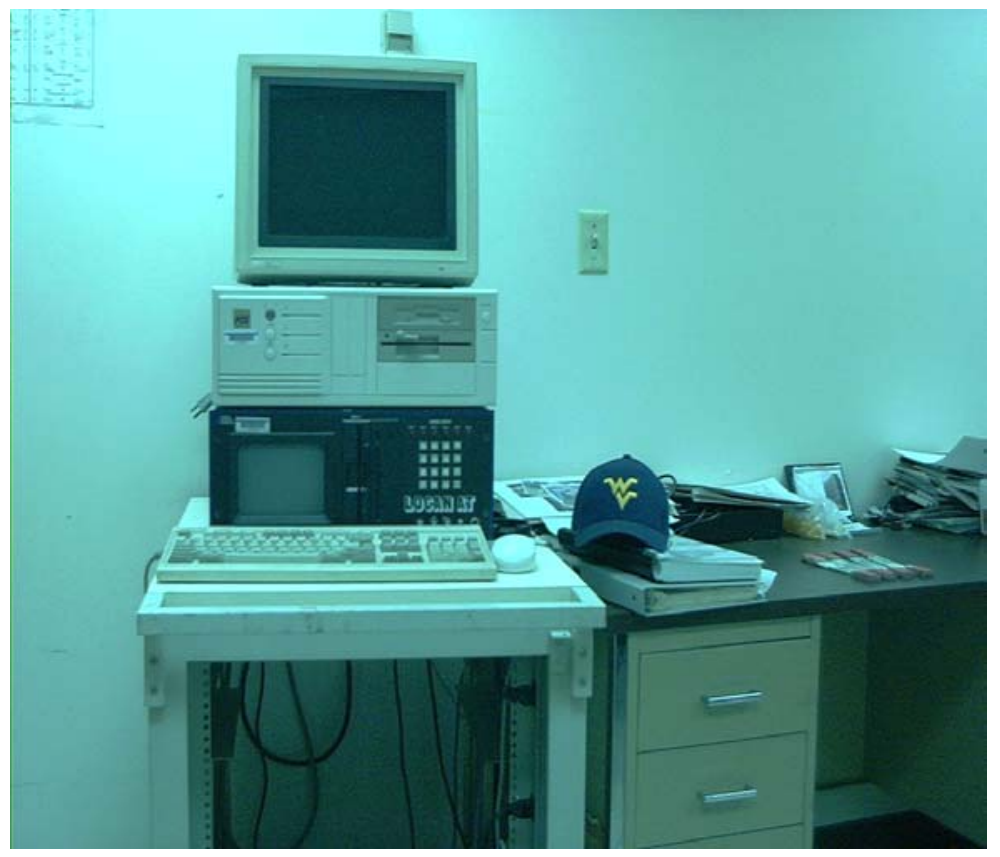

Figure 3.4 LOCAN AT and PC

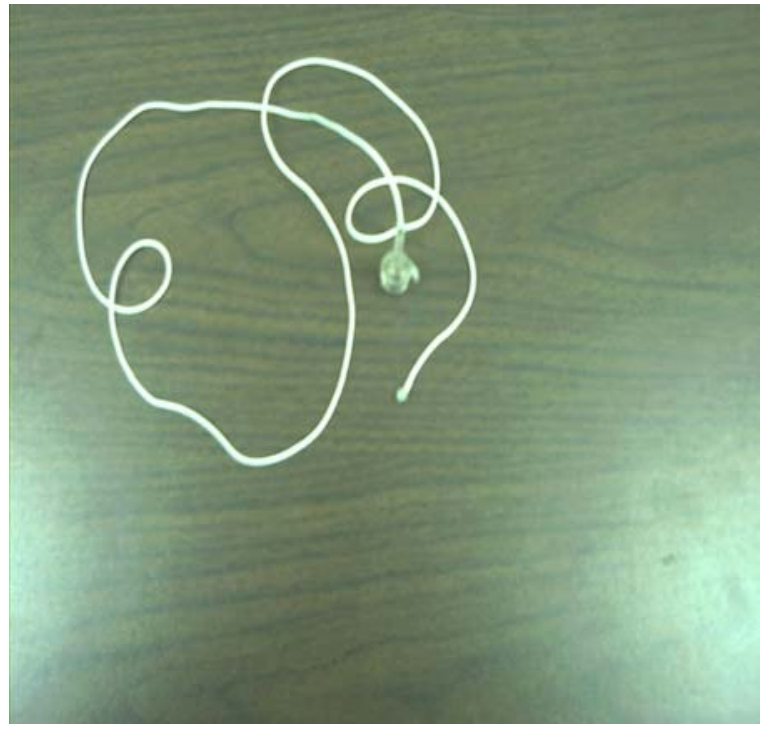

(a)

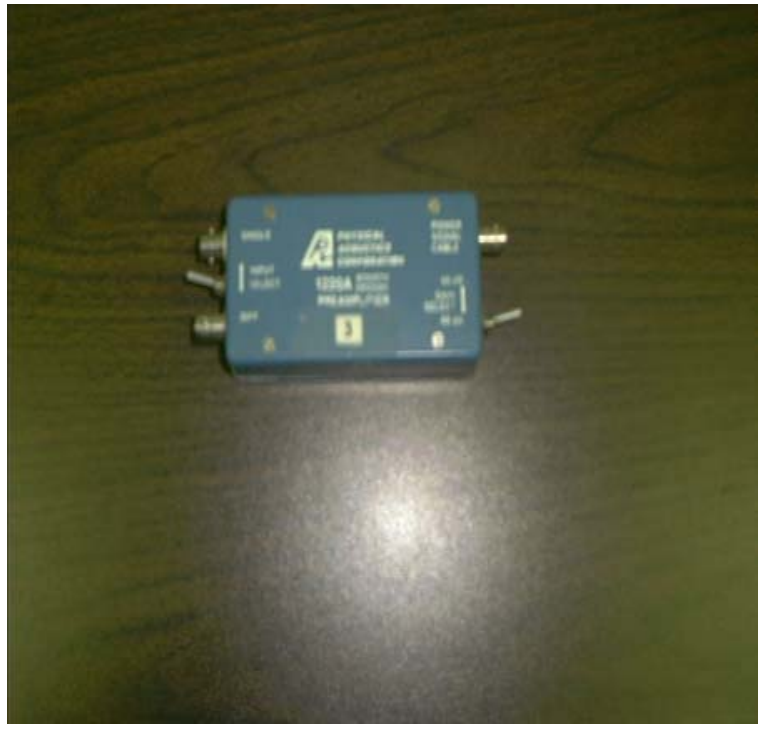

(b)

Figure 3.5 (a) PICO Sensor and (b) Model 1220A Preamplifier 


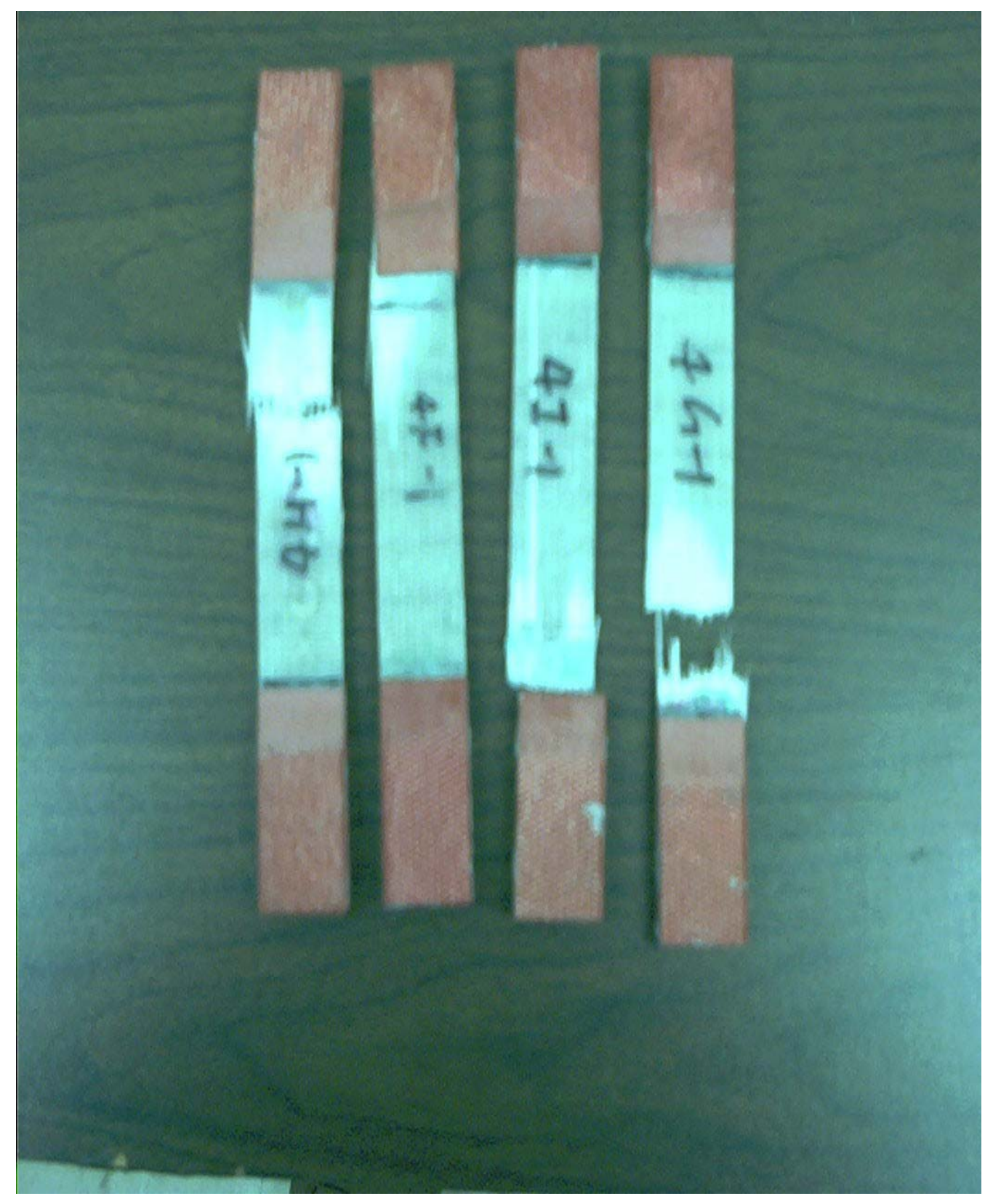

Figure 3.6 Failed Tension Test Specimens (tests 4t-7t) 


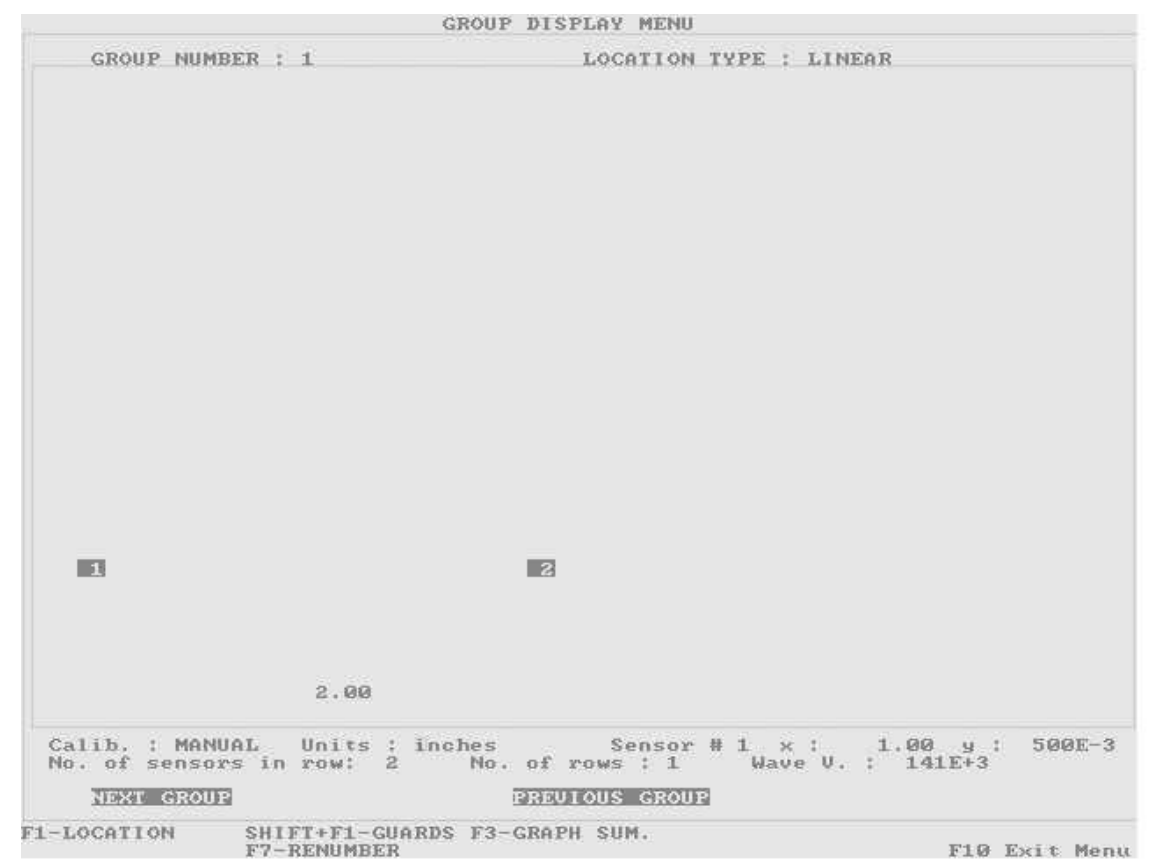

Figure 3.7 Sensor Layout Screen

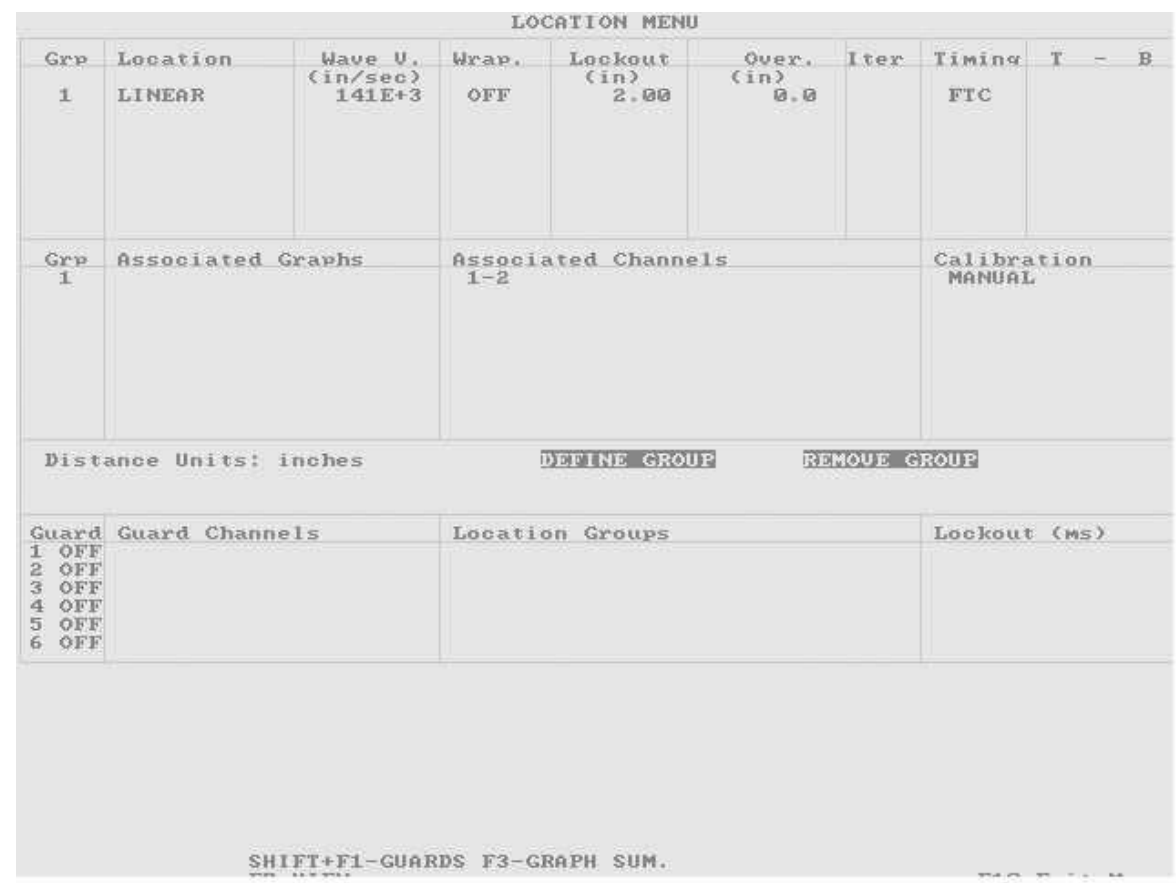

Figure 3.8 2-D Location Setup Screen 


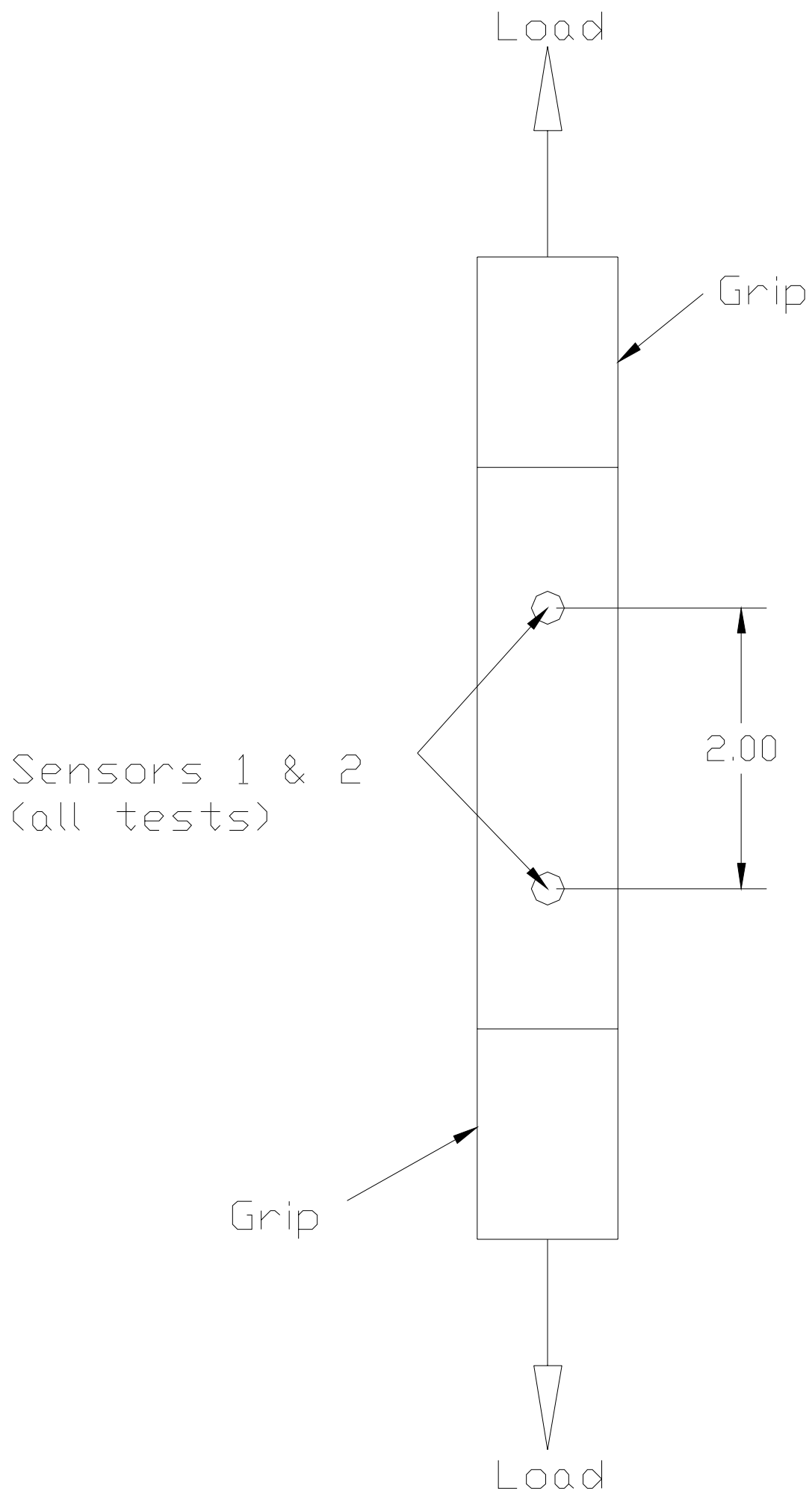

Figure 3.9 Sensor Layout (all tests) 


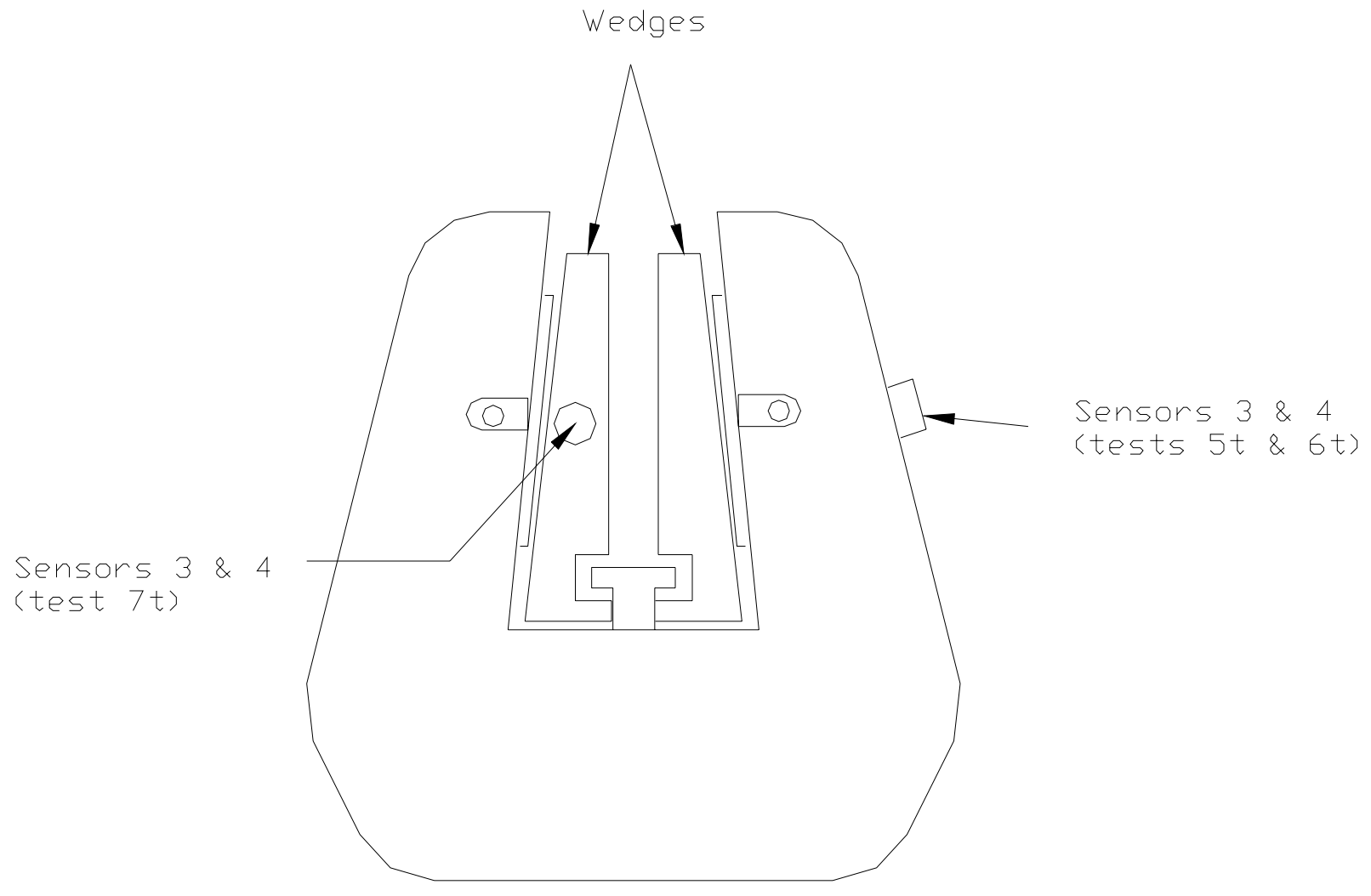

Figure 3.10 Grip with Additional Sensors (tests 5t, 6t, 7t) 


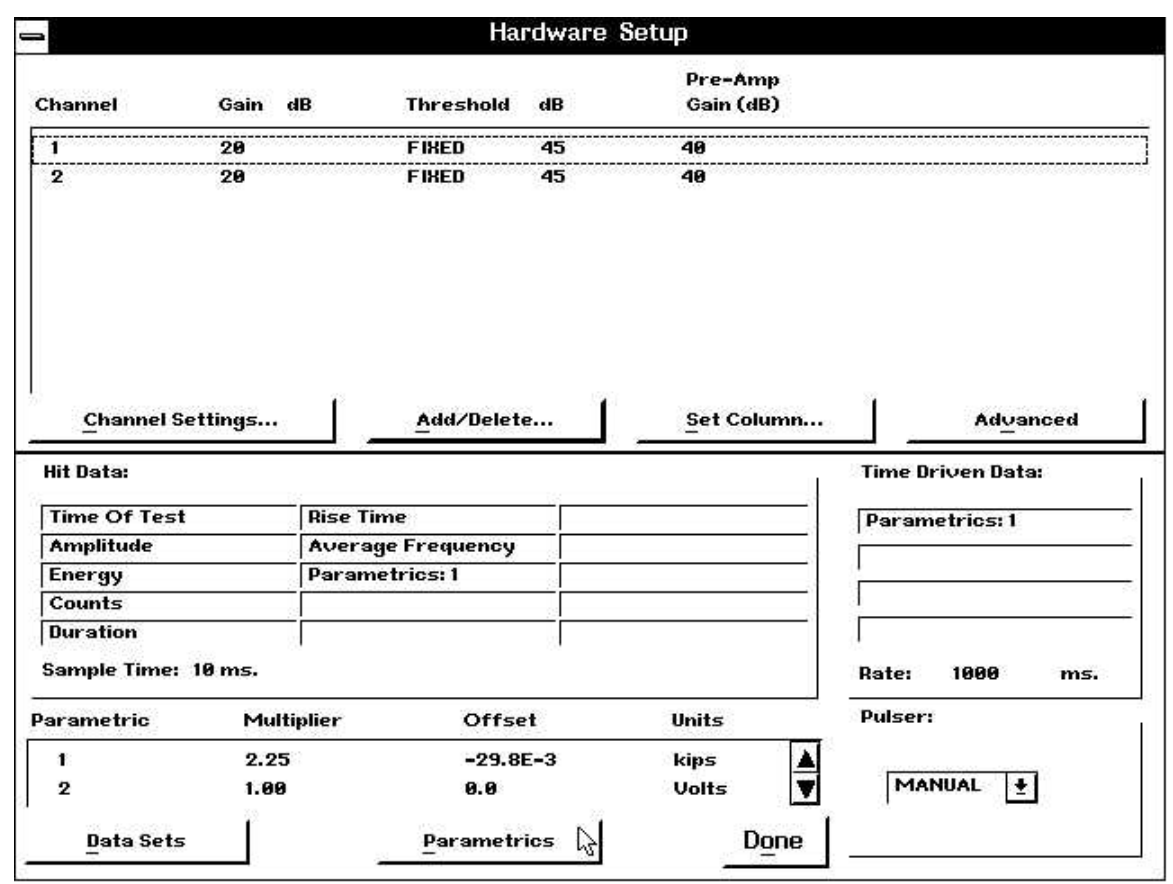

Figure 3.11 Hardware Setup Screen

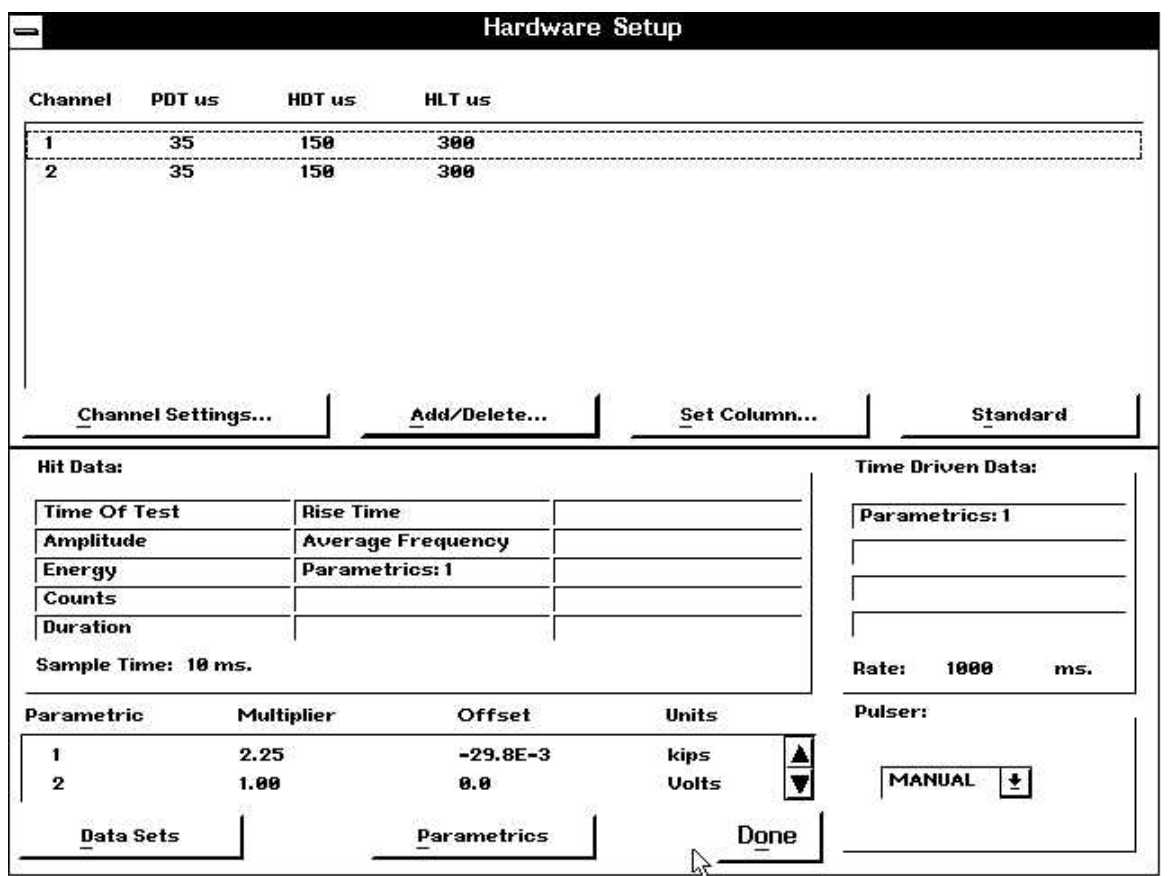

Figure 3.12 Advanced Hardware Setup Screen 


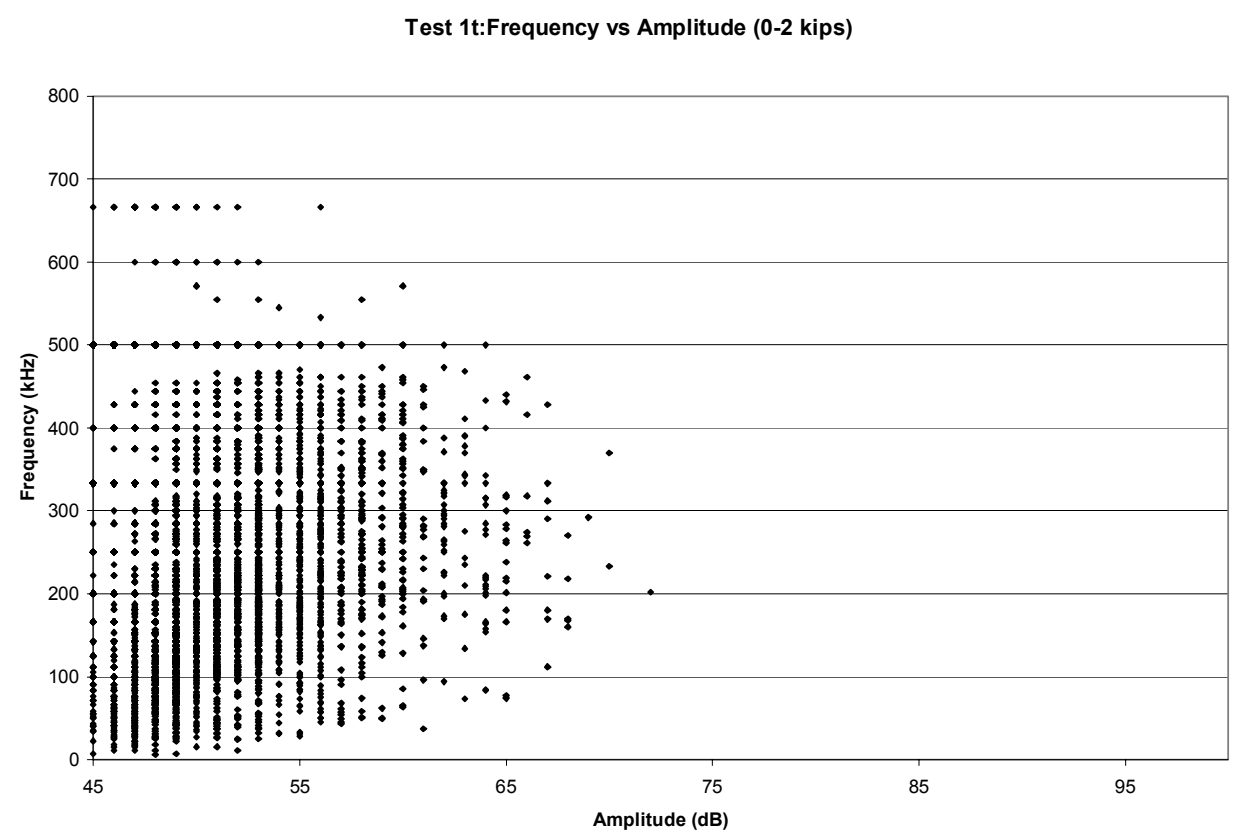

(a)

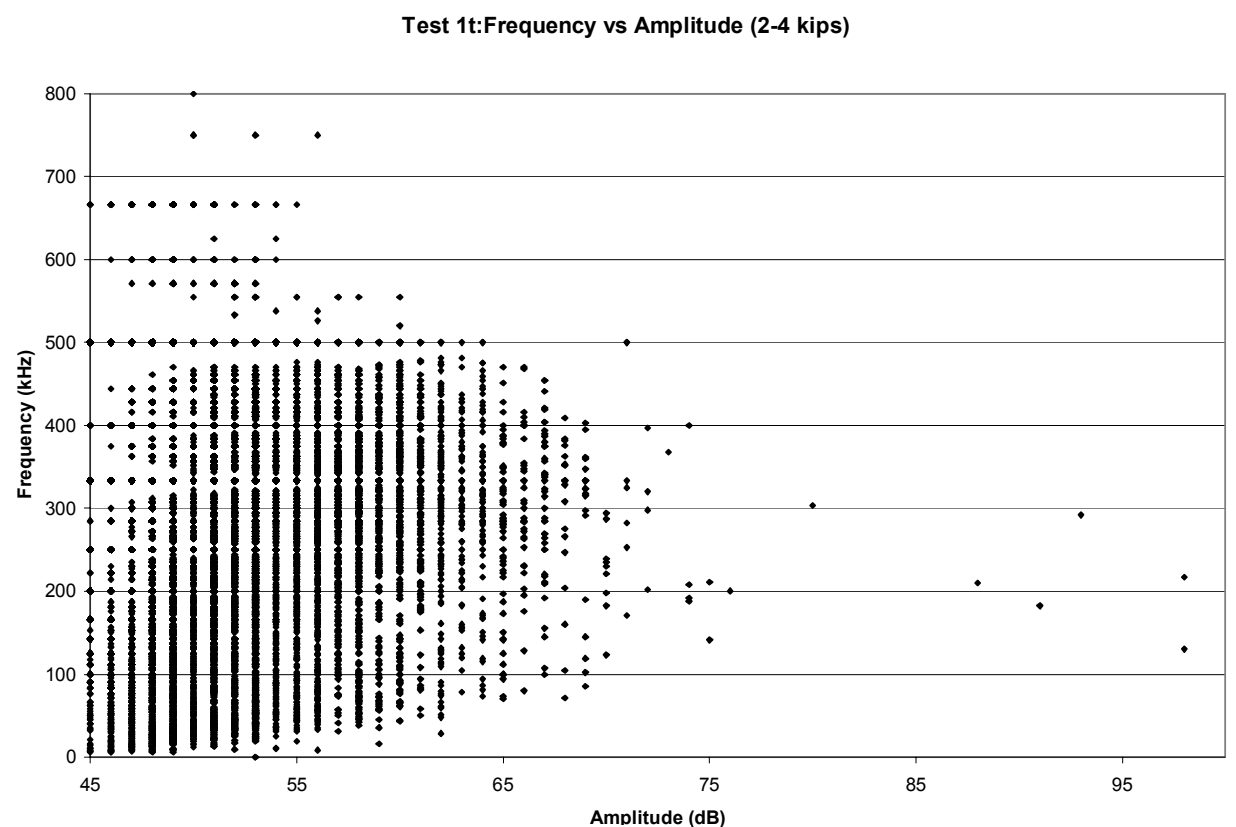

(b)

Figure 3.13 Test 1 t Frequency vs. Amplitude (a) $1^{\text {st }}$ quarter (b) $2^{\text {nd }}$ quarter 


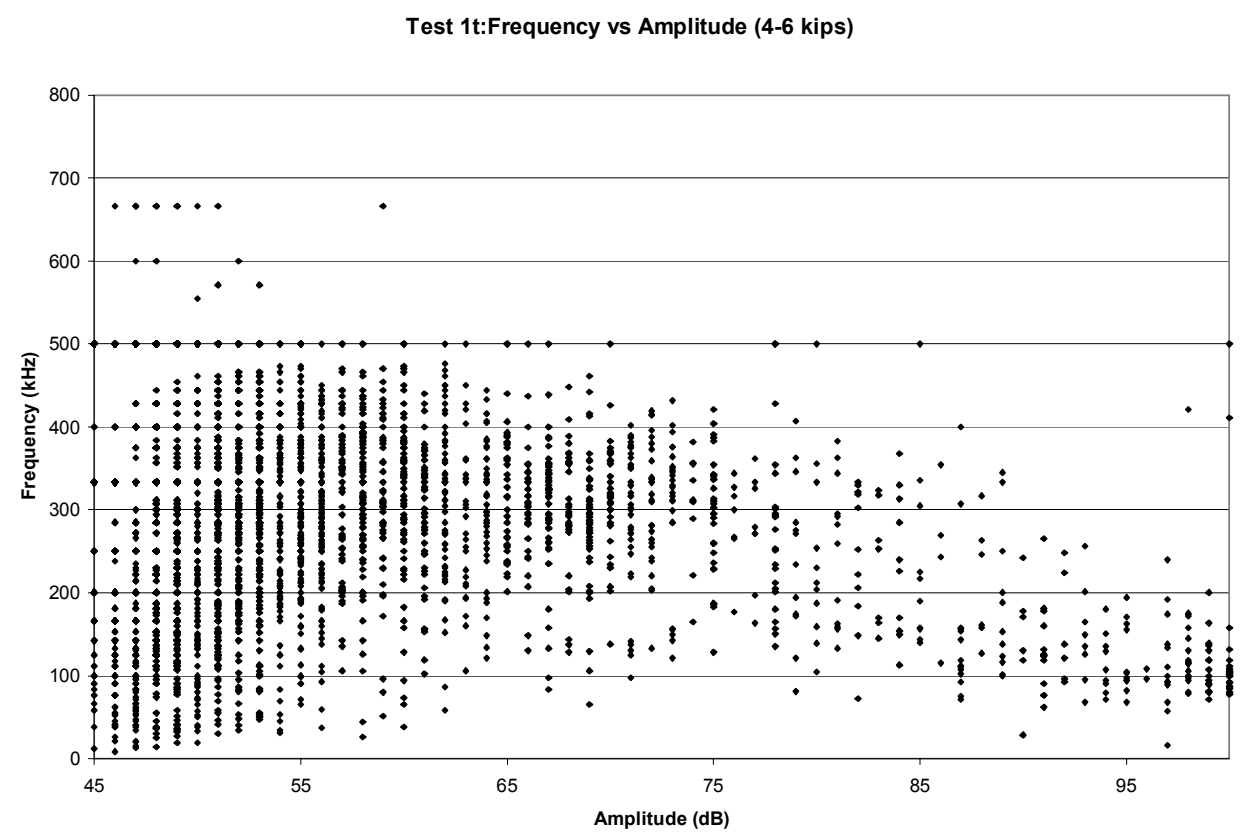

(c)

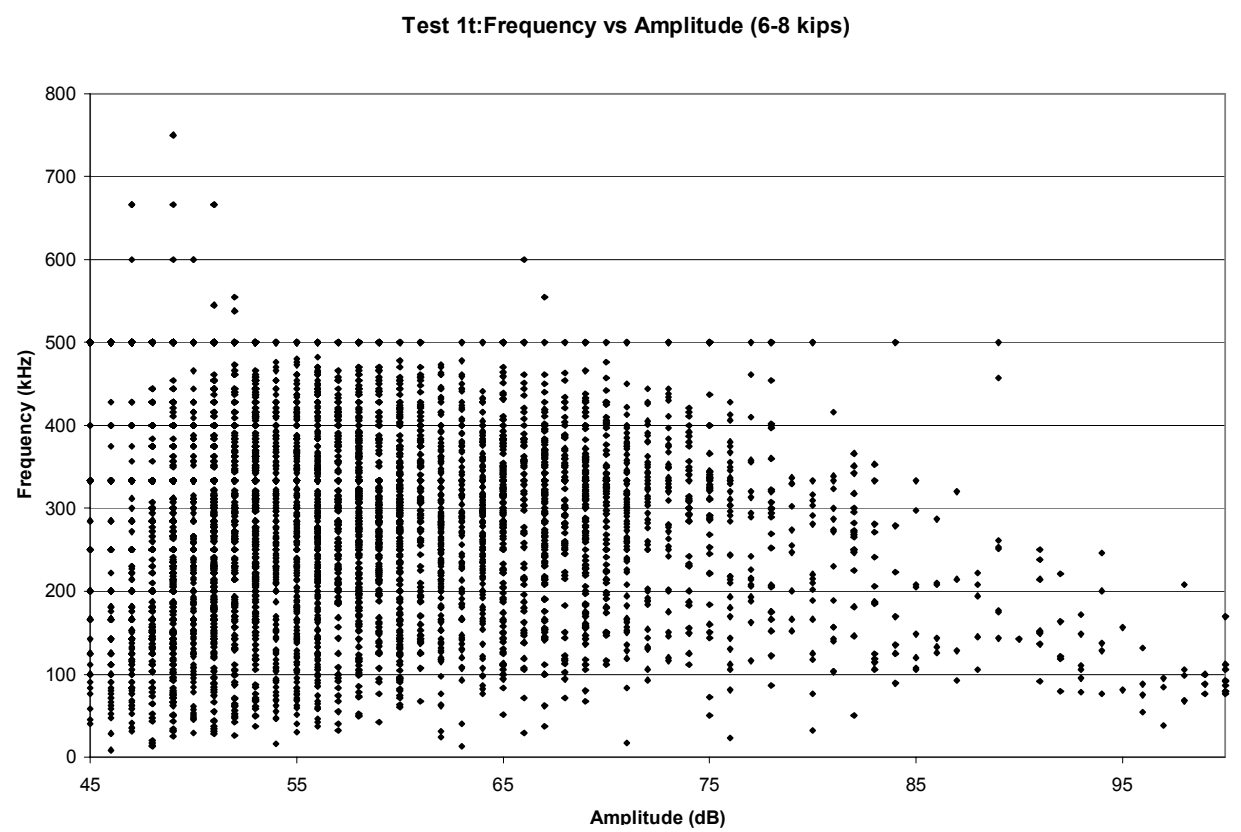

(d)

Figure 3.13 cont. (c) $3^{\text {rd }}$ quarter (d) $4^{\text {th }}$ quarter 


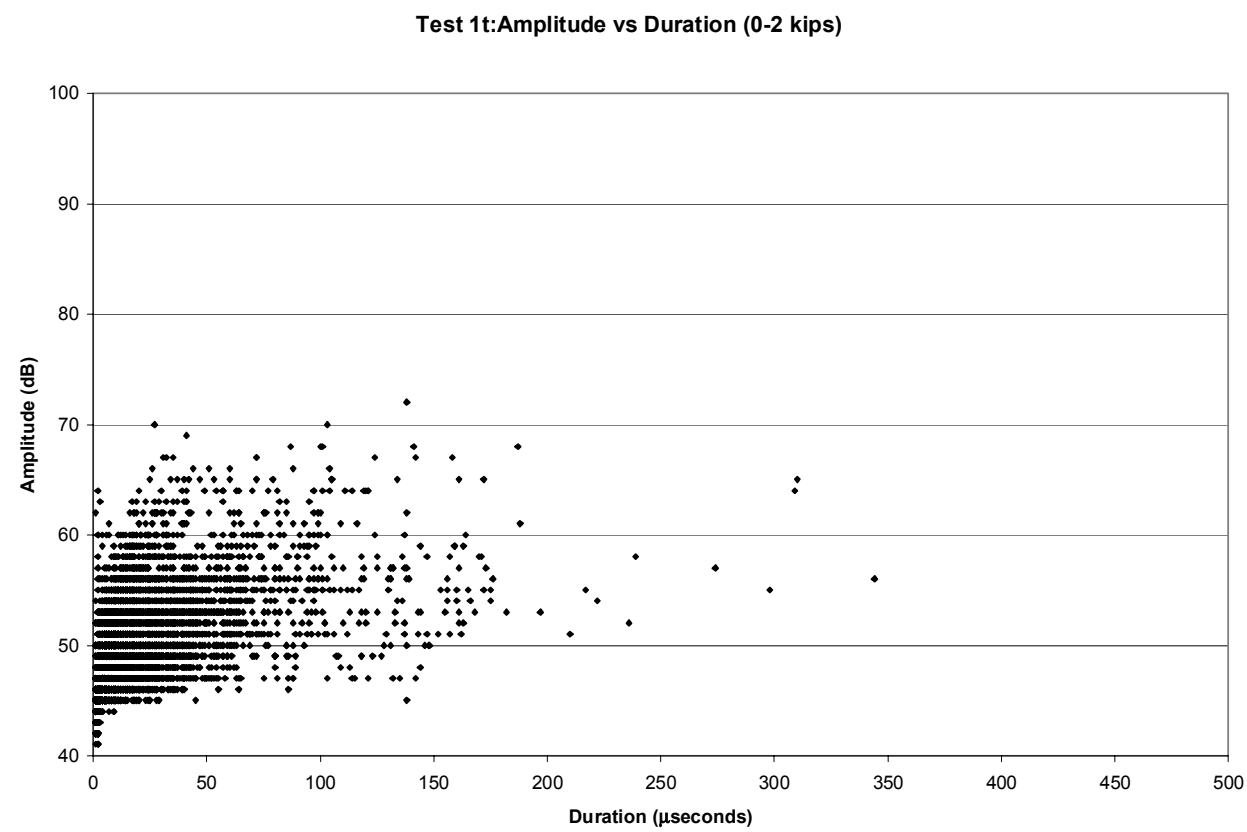

(a)

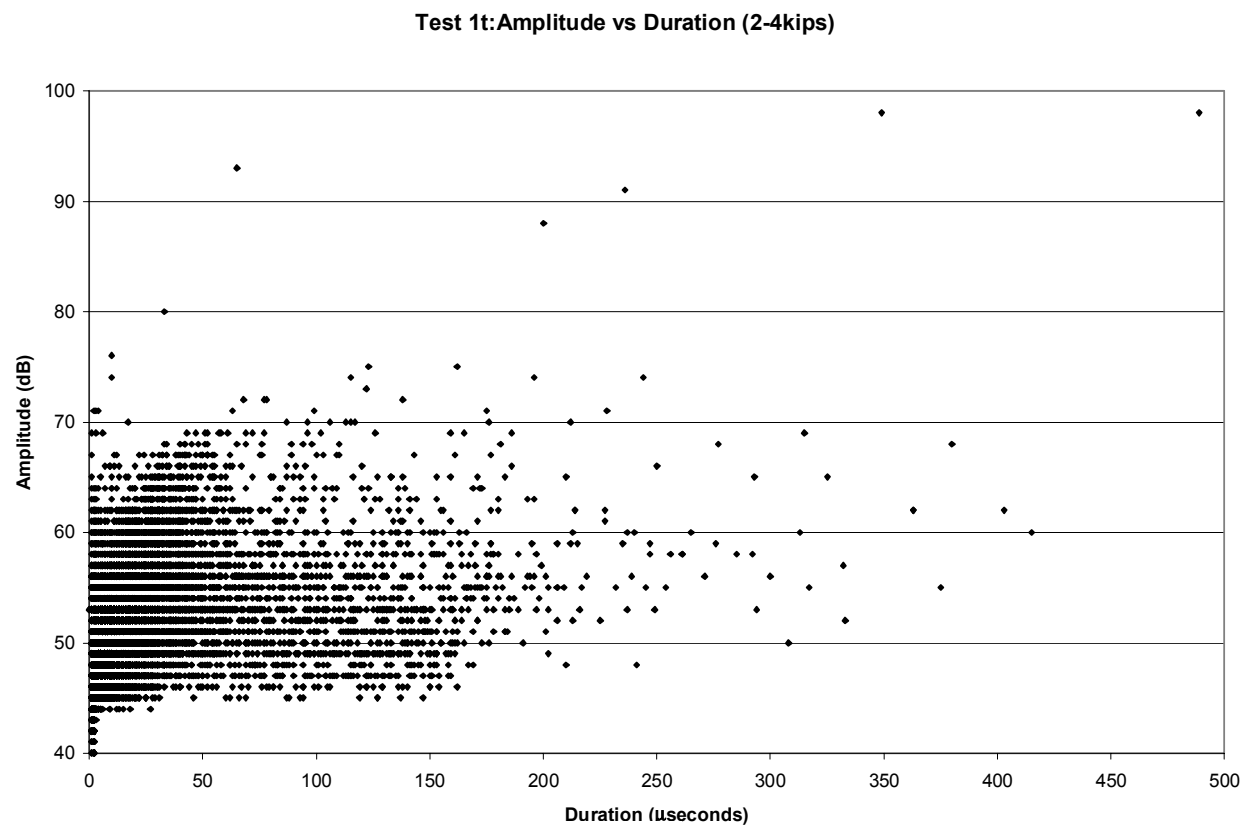

(b)

Figure 3.14 Test 1 t Amplitude vs. Duration (a) $1^{\text {st }}$ quarter (b) $2^{\text {nd }}$ quarter 


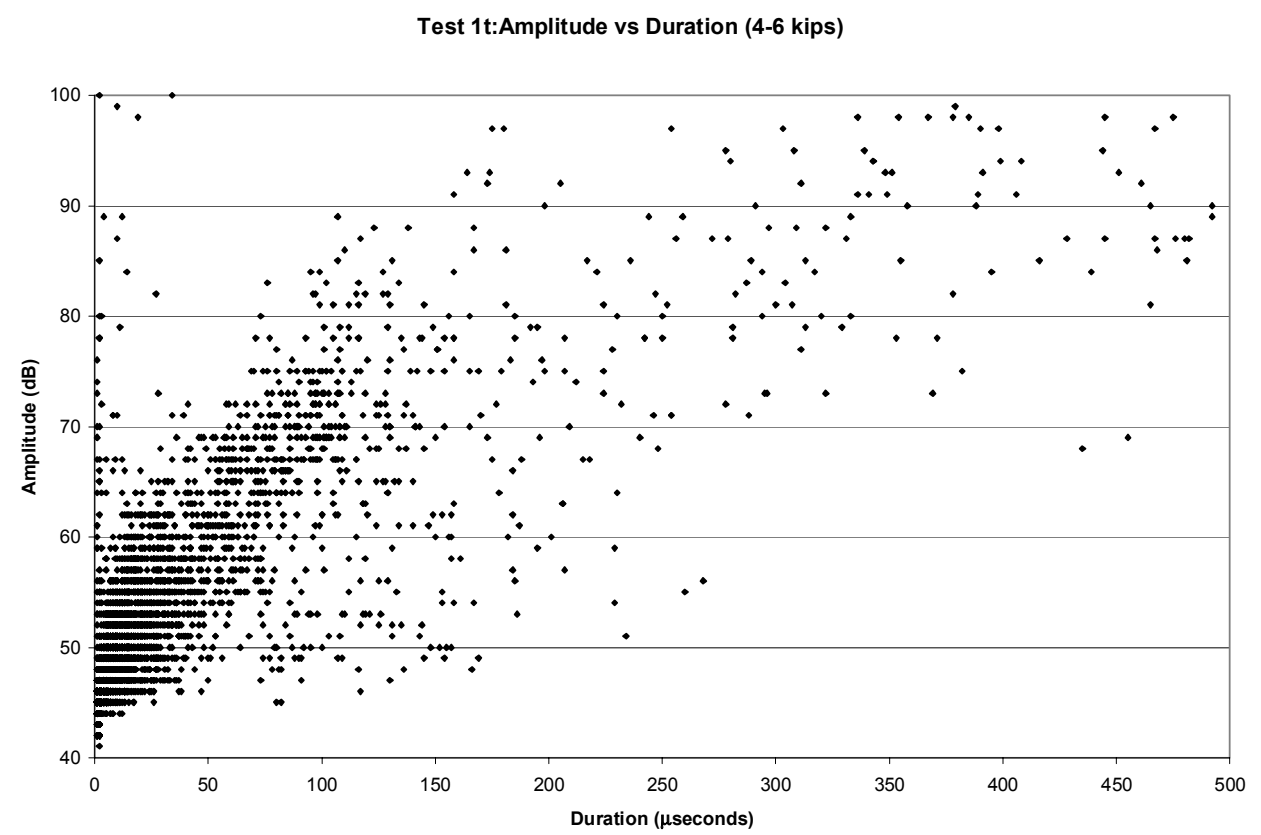

(c)

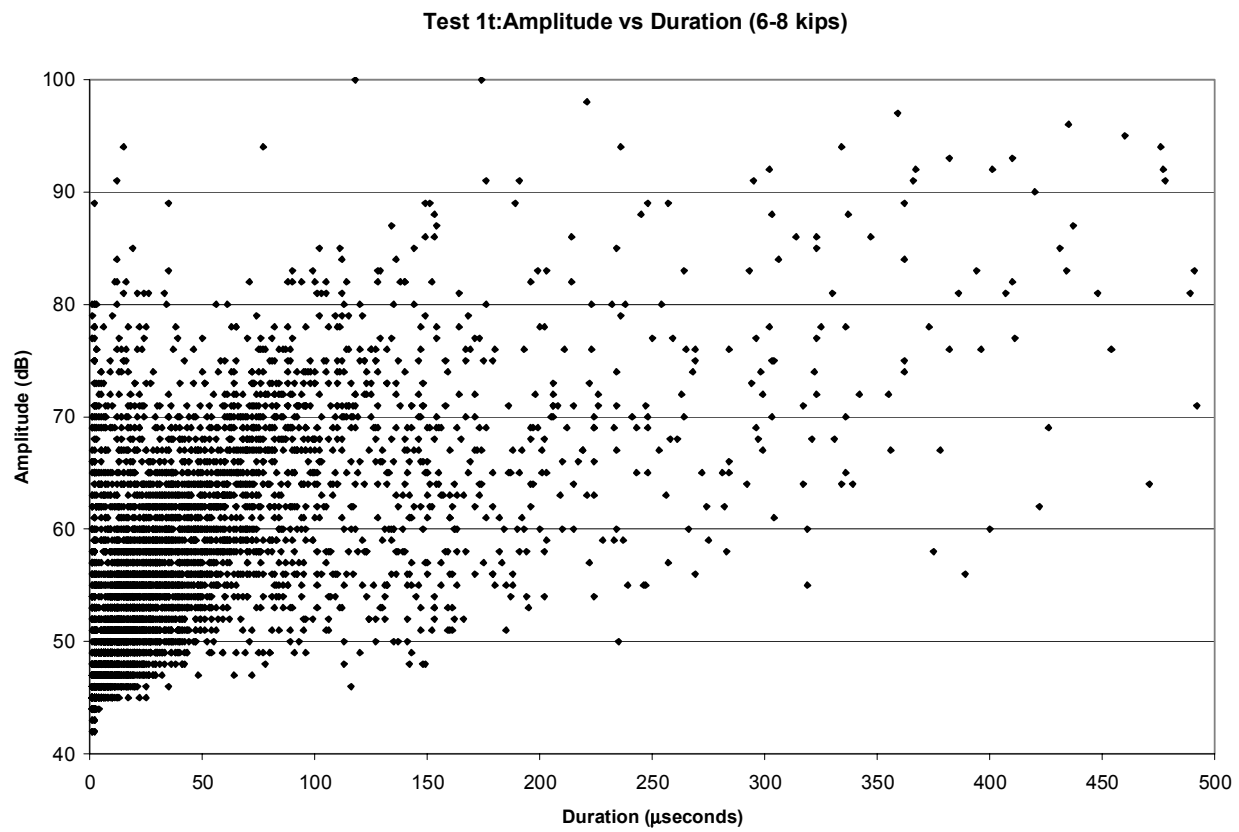

(d)

Figure 3.14 cont. (c) $3^{\text {rd }}$ quarter (d) $4^{\text {th }}$ quarter 


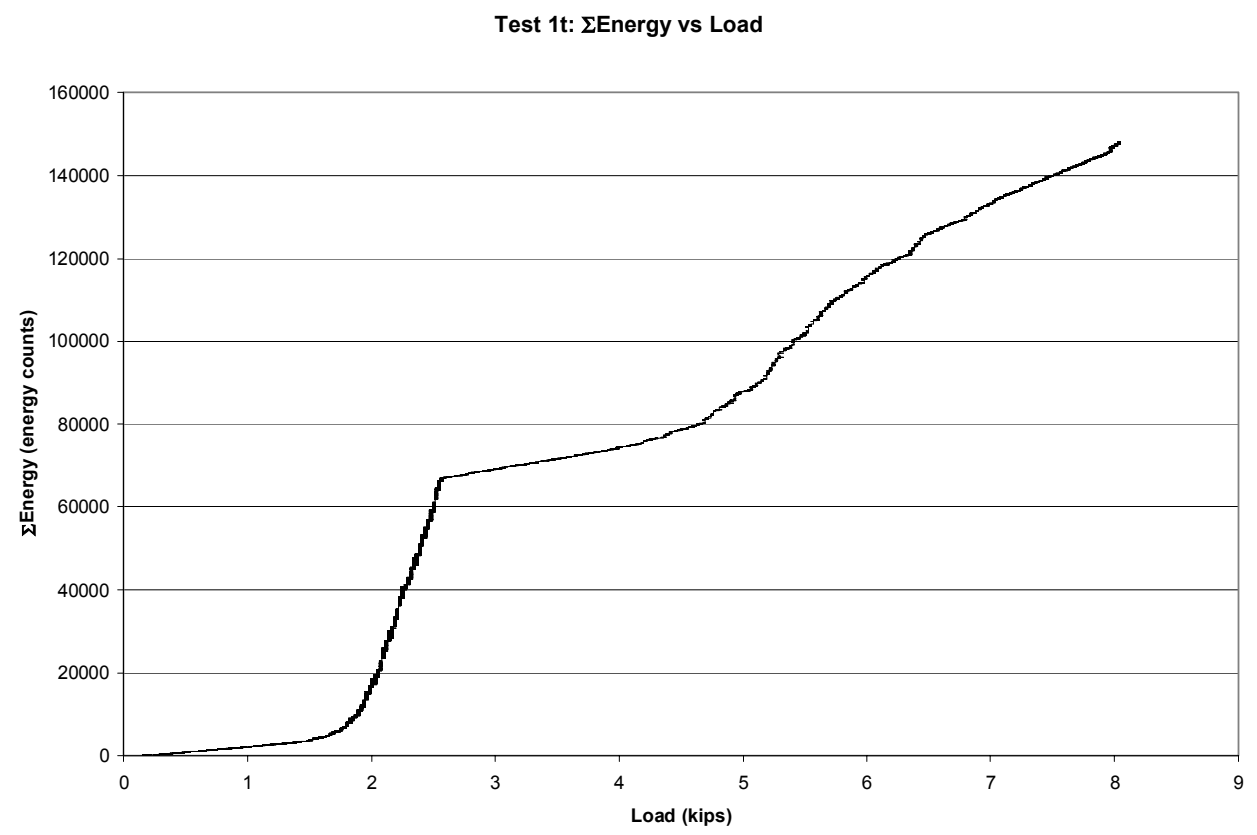

(a)

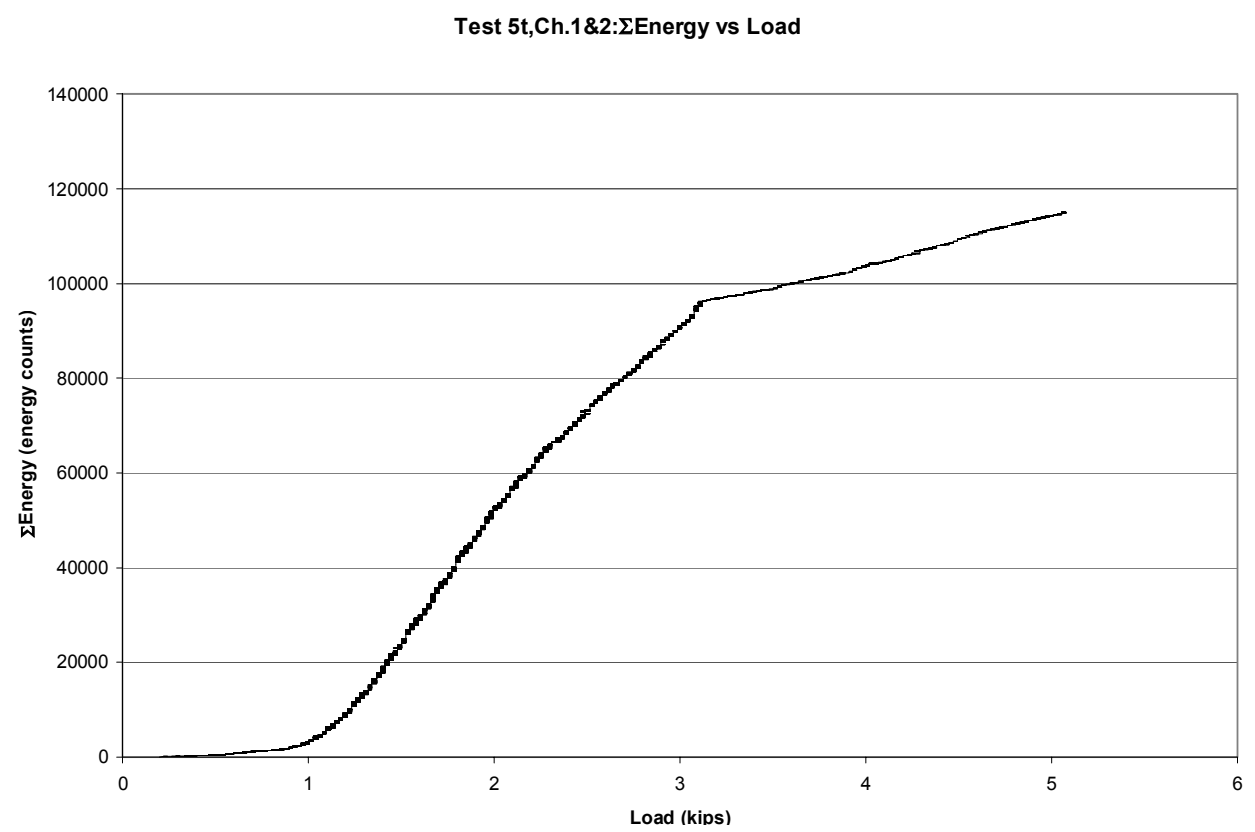

(b)

Figure 3.15 $\Sigma$ Energy vs. Load (a) test $1 \mathrm{t}$ (b) test $5 \mathrm{t}$ 


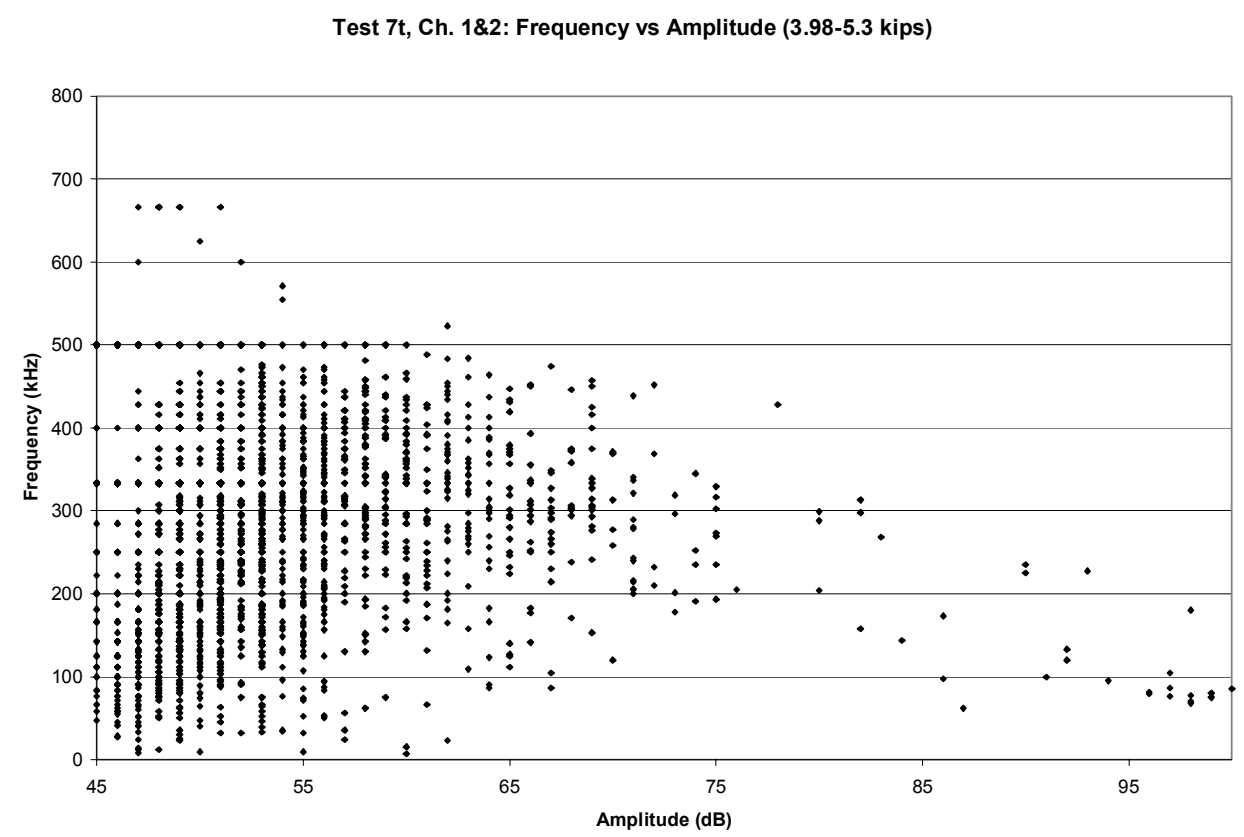

(a)

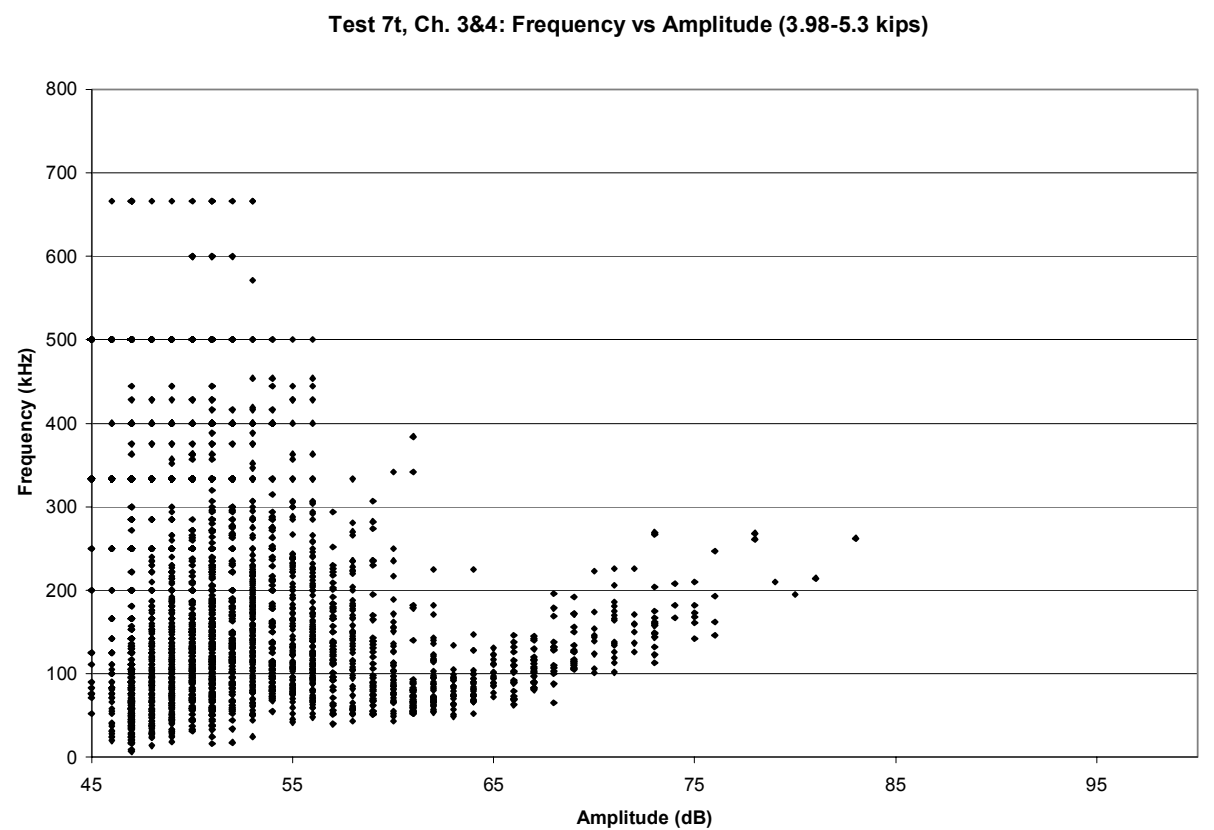

(b)

Figure 3.16 Test $7 \mathrm{t}$ Frequency vs. Amplitude $4^{\text {th }}$ quarter (a) ch. $1 \& 2$ (b) ch. $3 \& 4$ 


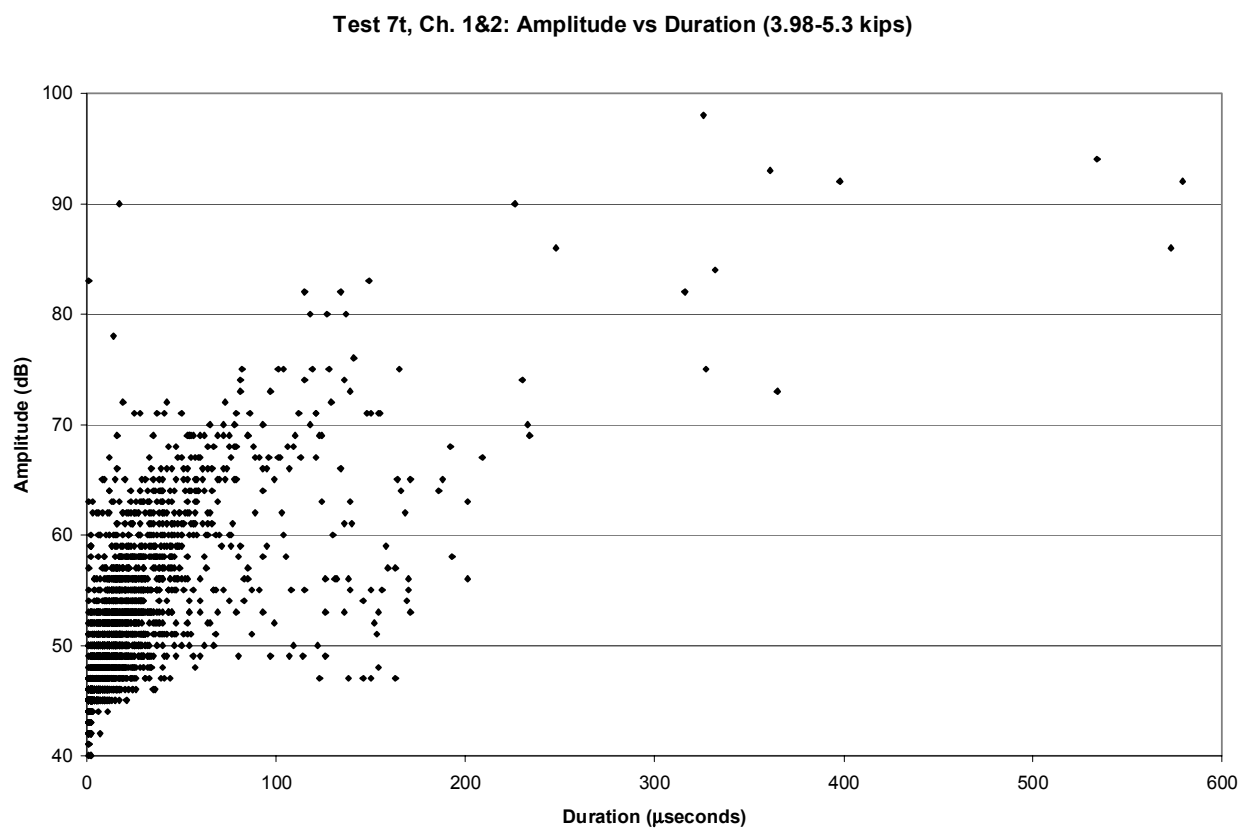

(a)

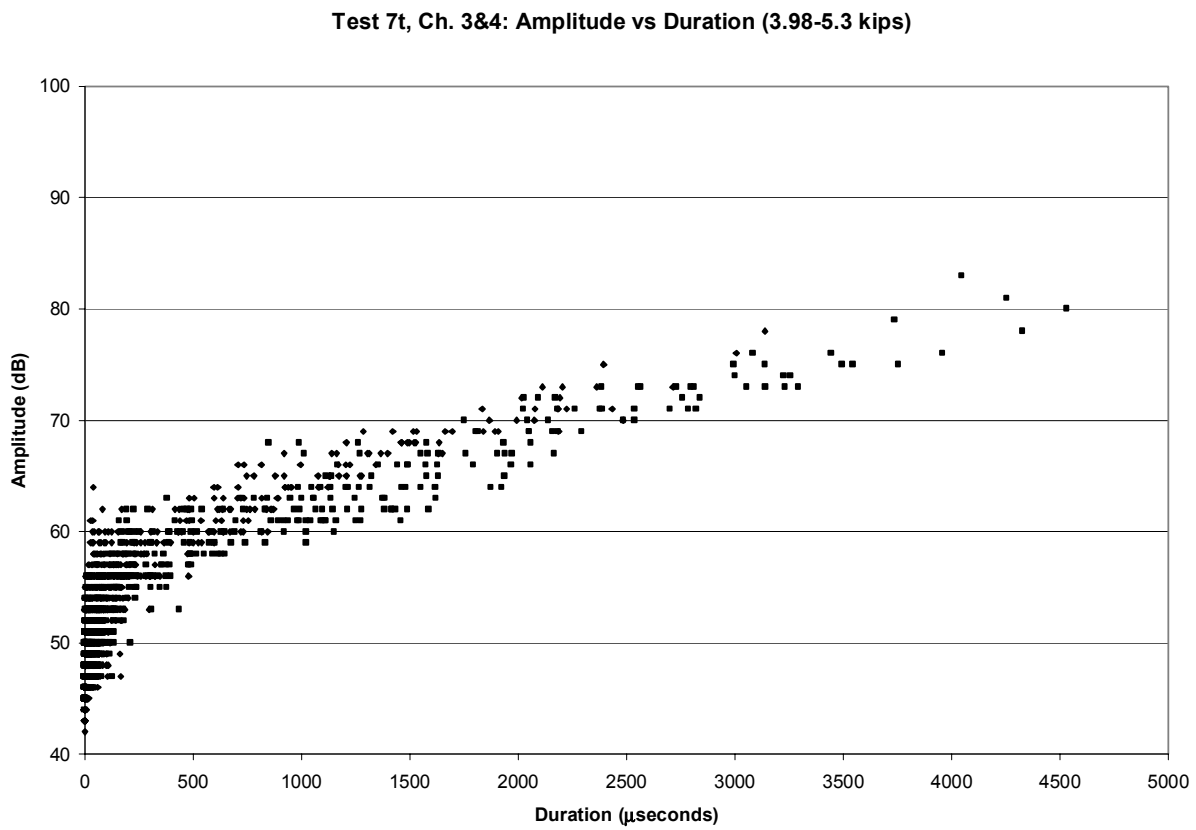

(b)

Figure 3.17 Test $7 \mathrm{t}$ Amplitude vs. Duration $4^{\text {th }}$ quarter (a) ch. $1 \& 2$ (b) ch. $3 \& 4$ 


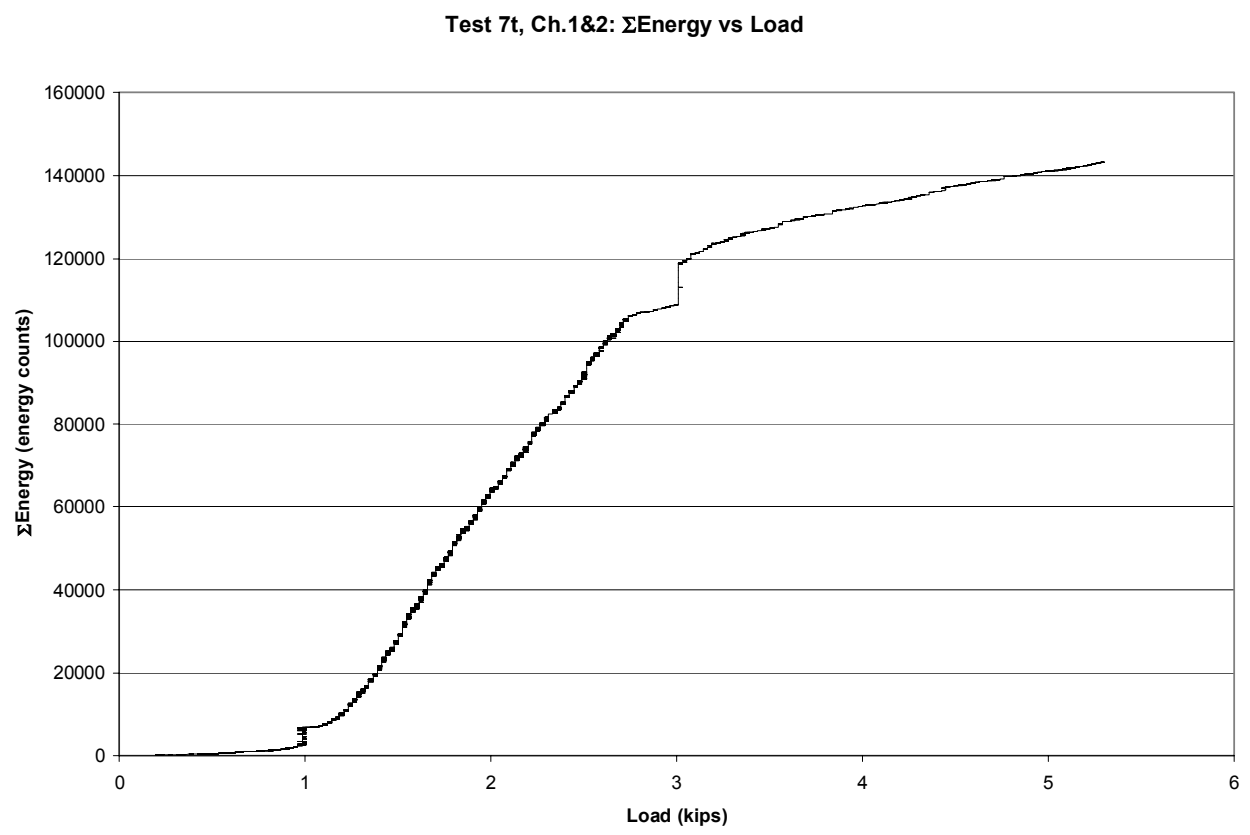

(a)

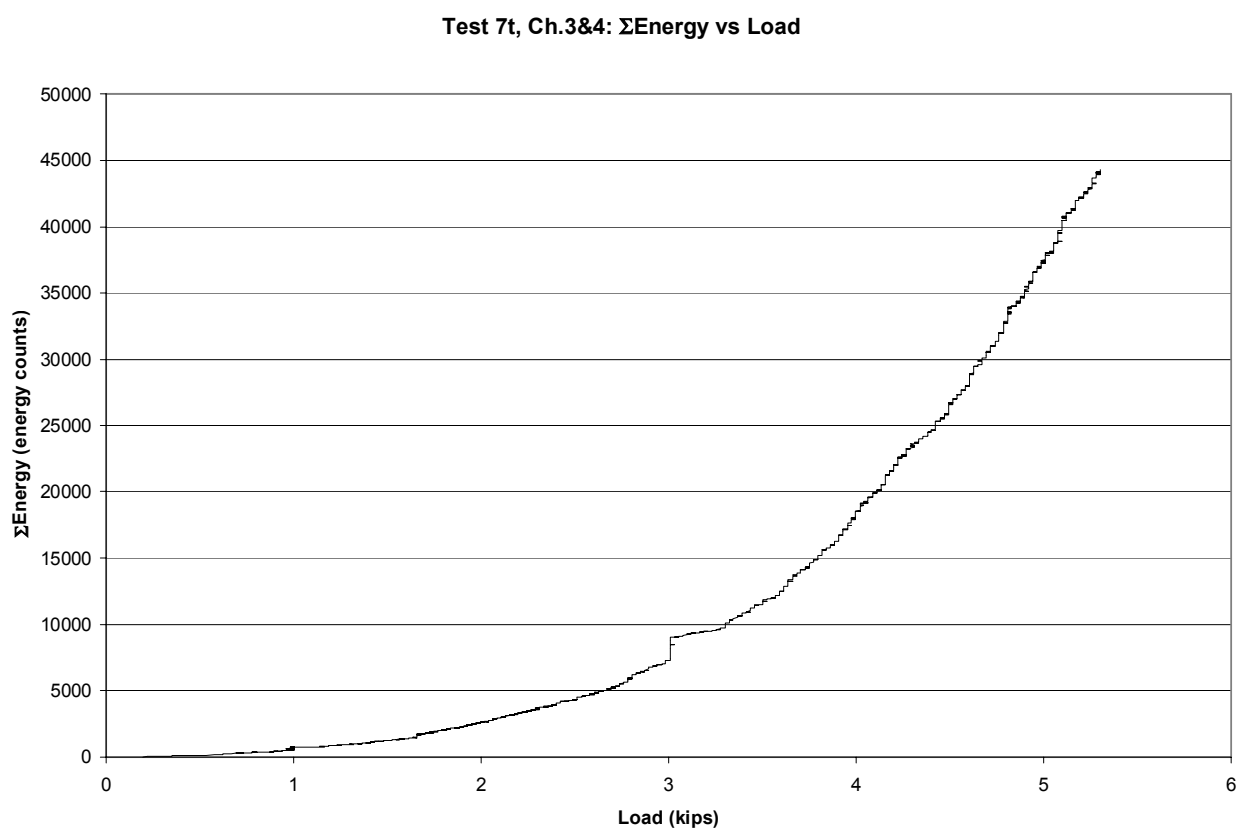

(b)

Figure 3.18 Test $7 \mathrm{t} \Sigma$ Energy vs. Load (a) ch. $1 \& 2$ (b) ch. $3 \& 4$ 


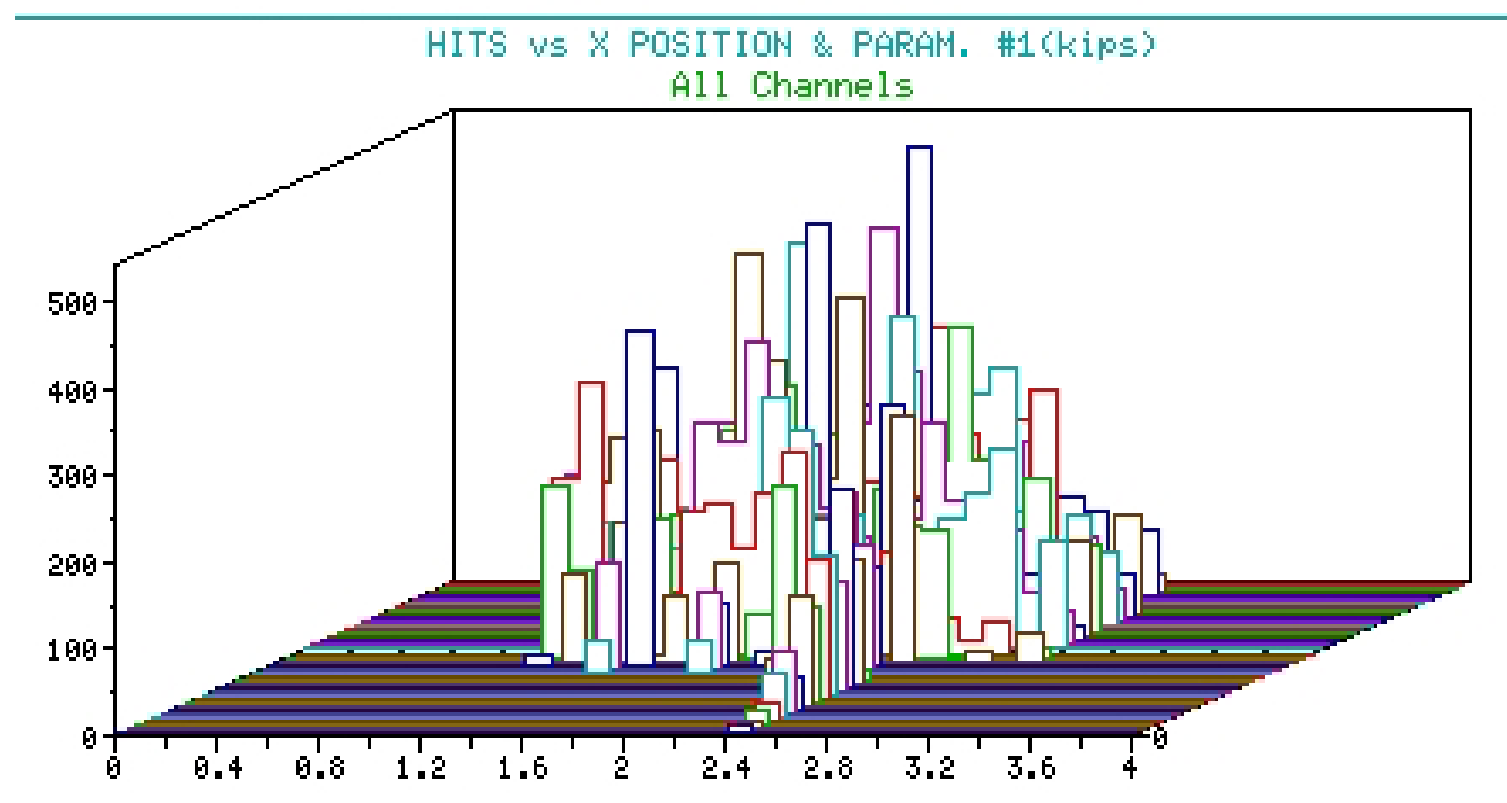

(a)

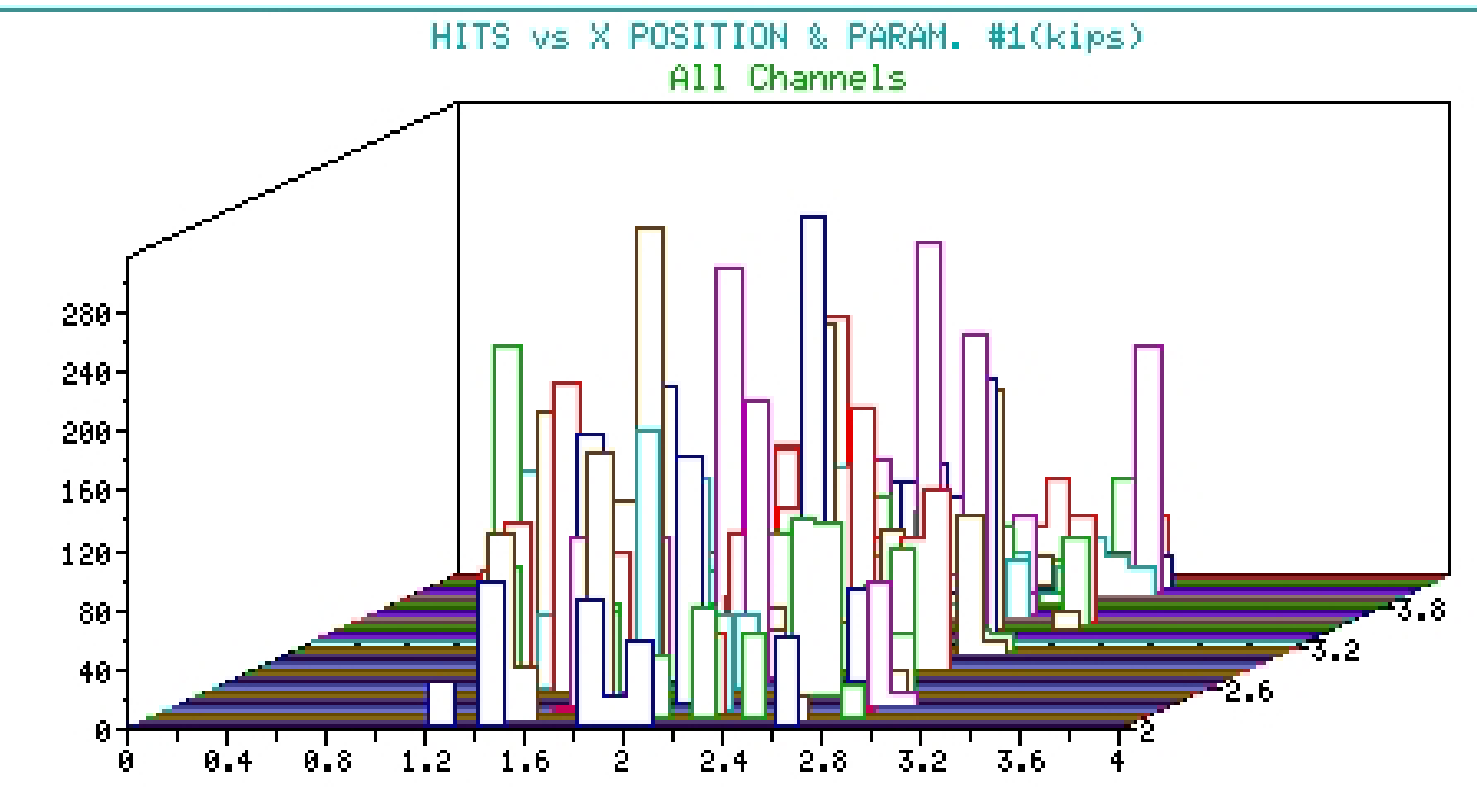

(b)

Figure 3.19 Test 4t Hits vs. Position vs. Load Unfiltered (a) 0-2 kips (b) 2-5.3 kips 


\begin{tabular}{|c|c|c|c|c|}
\hline Time & Sensor & Location & Sum 1 & Sum 1 \\
\hline (seconds) & (number) & & (sum) & (sum) \\
\hline & & & & \\
\hline $\mathrm{t} 1$ & 1 & & 0 & 0 \\
\hline $\mathrm{t} 2$ & 1 & same & 0 & 0 \\
\hline $\mathrm{t} 3$ & 2 & 1 & 1 & 0 \\
\hline $\mathrm{t} 4$ & 2 & same & 1 & 0 \\
\hline $\mathrm{t} 5$ & 2 & same & 1 & 0 \\
\hline $\mathrm{t} 6$ & 2 & same & 1 & 0 \\
\hline $\mathrm{t} 7$ & 1 & 2 & 1 & 1 \\
\hline $\mathrm{t} 8$ & 1 & same & 1 & 1 \\
\hline $\mathrm{t} 9$ & 2 & 1 & 2 & 1 \\
\hline $\mathrm{t} 10$ & 2 & same & 2 & 1 \\
\hline
\end{tabular}

Figure 3.20 Example of Sum of First Sensor Hit Plot Construction 
Test 2t: Sum of 1st Sensor Hit

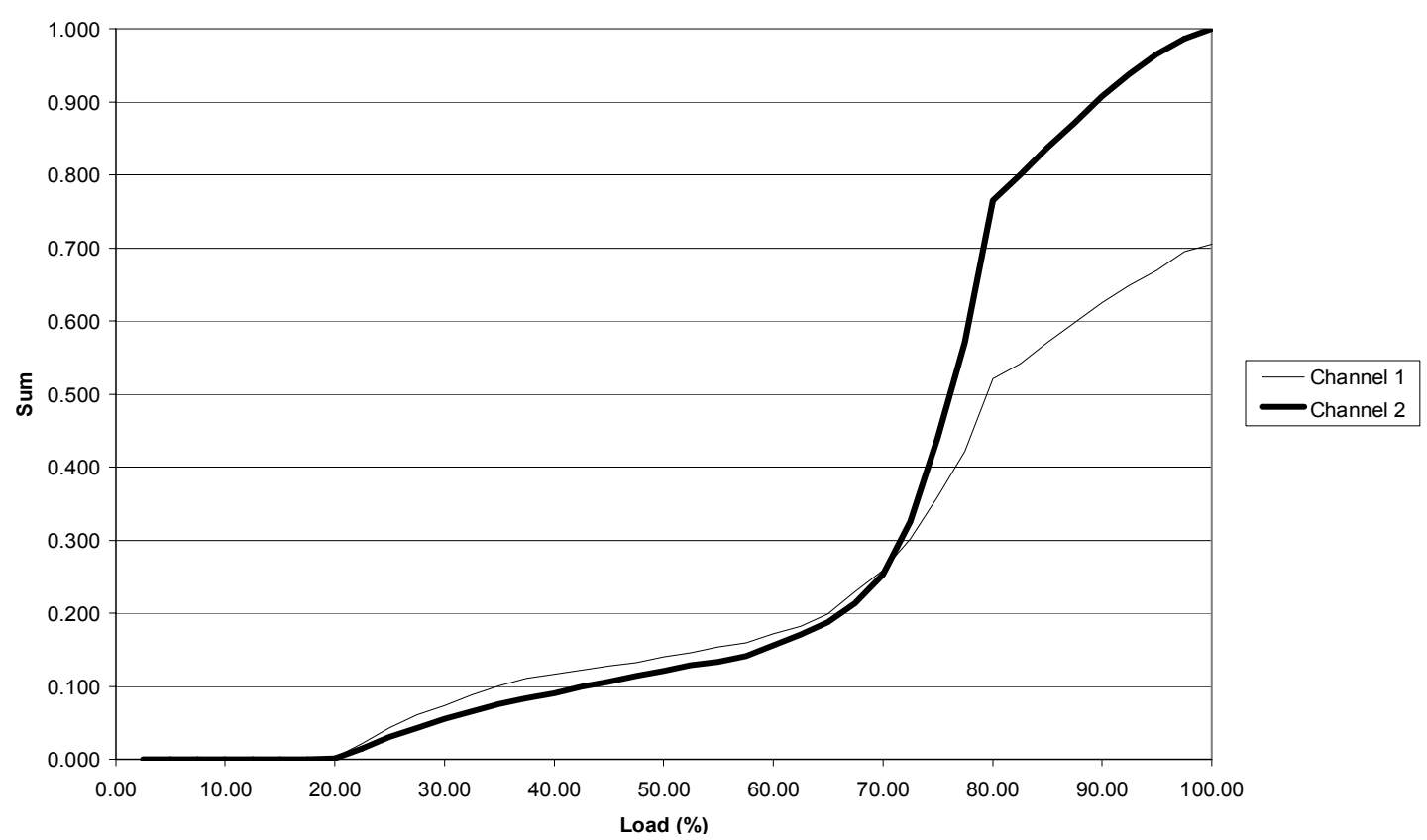

(a)

Test 4t: Sum of 1st Sensor Hit

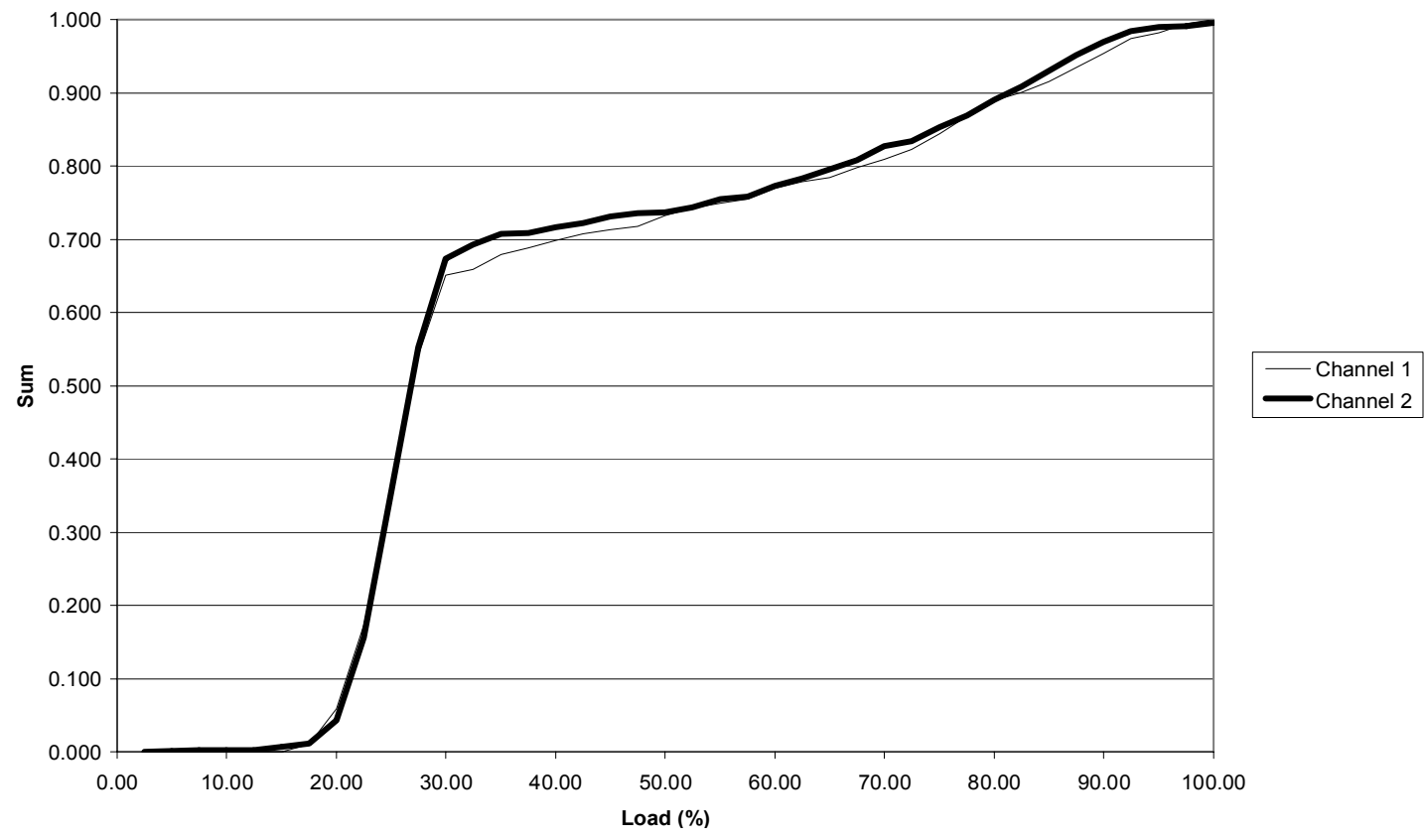

(b)

Figure 3.21 Sum of $1^{\text {st }}$ Sensor Hit vs. Load (a) test $2 \mathrm{t}$ (b) test $4 \mathrm{t}$ 


\section{Chapter 4}

\section{Bending Tests}

\subsection{Specimens}

Bending tests were conducted on specimens cut from actual sections of bridge deck. These specimens were made according to the dimensions set forth in ASTM D 790-92 for four point bending tests at third points. This standard also required that five specimens of each type were cut. These groups of five were labeled group 1, group 2, and group 3. The first five specimens (group 1) were from a deck section produced by Bedford Reinforced Plastics Inc. The measured dimensions for these specimens were $1 / 4$ inch thick by $1 / 2$ inch wide with a support span of 8 inches. This resulted in a $\mathrm{L} / \mathrm{d}$ ratio of 32 to 1 . A minimum of 1 inch overhang was required past each support point along with a 2.67 in load span (1/3 of the support span). The next 5 specimens (group 2) were cut from a section of Superdeck from Creative Pultrusions. These specimens were also cut to $1 / 2$ inch wide, but were slightly thinner at $.20 \mathrm{inch}$. The support span used was kept at the same 8 inches so as to utilize the same loading set-up. The resulting $\mathrm{L} / \mathrm{d}$ ratio was 40 to 1 . The last 5 samples (group 3) were also from a Bedford Reinforced Plastics Inc. product. These particular specimens were cut from a plate specimen with a different internal architecture than the specimens cut from the Bedford Reinforced Plastics Inc. deck section. The specimens cut from the plate measured $1 / 2$ inch thick by $1 / 2$ inch wide with a support span of 8 inches. The resulting $\mathrm{L} / \mathrm{d}$ ratio was 16 to 1 . A picture of the specimens can be seen in Figure 4.1. The Bedford deck piece (group 1) is at the top, Creative deck piece (group 2) in the middle, and Bedford plate piece (group 3) at the bottom. Note that the loading rate suggested in ASTM D 790-92 was not applied. Instead, a slower loading rate was used so that an ample amount of AE signals could be generated. 


\subsection{Instruments and Equipment}

\subsubsection{INSTRON Loading Frame}

The loading frame used for these bending tests was an INSTRON model 1331. The actuator, which has a 2.6 in diameter, is powered by hydraulic pressure generated by the MTS (Material Testing System) power supply. The MTS power supply has a maximum pressure capacity of 3,000 psi. The maximum displacement of the frame is 5 inches, and the maximum load of 10 kips can be applied in either tension or compression. A 22 kip INSTRON load cell is used to measure the load that is being applied. The frame also allows for the loading to be controlled by load or displacement. Figure 4.2 is a picture of the INSTRON loading frame, actuator, controller, and hydraulic power supply.

\subsubsection{Computer Controller}

The loading to the INSTRON 1331 loading frame is controlled by a MTS model 407 digitally-supervised servo controller. The controller allows for testing to be done with either load or displacement as the control. Controls of loading magnitude, waveform, and frequency are also present. In addition to these features, the model 407 has interlocks for adjustable upper/lower limit, underpeak, error detection for each installed conditioner module, and end-ofcount. As stated before, this controller can be seen in Figure 4.2.

\subsubsection{LVDT}

For these bending tests, the additional parameter of displacement was also collected. In order to do this a LVDT was used. The LVDT used was a model 2000 HR-DC produced by 
Schaevitz Sensors. This DC powered LVDT has a nominal linear range of \pm 2 inches. This LVDT was powered by a BK Precision model 1660 triple output DC power supply. The model 2000 HR-DC was then connected to the LOCAN AT system.

Before the LVDT was used, it required calibration. The setup for this calibration can be seen in Figure 4.3. During calibration, a curve is developed by recording the voltage reading on the LOCAN AT system at displacements shown on the calibration tool. This curve can be seen in Figure 4.4. From this curve, a relation between the voltage and displacement can be seen. From this curve, the multiplier and offset was found. These were then input into the SPARTAN software of the LOCAN AT system to display the location.

\subsubsection{Acoustic Emission Equipment and Software Setup}

The $\mathrm{AE}$ acquisition system, software and equipment used were the same as those in Chapter 3.

\subsection{Test Procedures}

\subsubsection{Sensor Placement}

For all fifteen bending tests, the LOCAN AT's full capacity of six sensors was used. The first two sensors were placed on the tension side of the specimens directly below the two loading points and the second two sensors were placed on the compression side, between the support points and the loading points. Finally, the last two sensors were placed on the tension side, directly below the second two sensors. As in Chapter 3, the sensors were each attached using hot melt glue. Figure 4.5 is a schematic drawing of the sensor setup and specimen dimensions. 


\subsubsection{Test Setup}

In order to conduct four point bending test, a loading nose needed to be fabricated to transform the three point bending setup into a four point bending setup. This apparatus was made from a steel plate and round steel bars. Due to the fact that the four point loading nose was not connected to the loading frame, it added additional weight to the specimen. The loading nose was weighed and found to have a weight of $.81 \mathrm{lbs}$. Before starting each test, rubber pads were put under the loading noise and the supports to help in eliminating friction noise. The rubber used at the supports was replaced before each test. The LVDT was connected to the middle of the specimen to measure displacement. It was connected using a wooden dowel rod and rubber band so as to not increase the strength of the specimen.

\subsubsection{Software Setup}

Since different loading machines were used for the tension and bending tests, new multiplier and offset values were needed for the SPARTAN software. These were found using the same method as that shown in section 3.2.3 of Chapter 3. The multiplier value was found to be -100 and the offset 2.5 .

\subsubsection{General Test Procedures}

1. All AE sensors were mounted and connected to check for functionality. Dowel rod for LVDT connection was connected to specimen.

2. The LVDT and loading frame controller were connected to the LOCAN AT system to record parameters.

3. Rubber pads and specimen were placed on supports. 
4. Rubber pads and four point loading noise were placed on specimen.

5. LVDT core was attached to wooden dowel rod using fishing line.

6. Data acquisition system was started and loading began.

7. Specimen was loaded to failure and data was collected.

Figure 4.6 is a picture of specimen B10 being prepared to be loaded. In this picture, the LVDT can also be seen. Figure 4.7 shows the same specimen immediately before it broke.

\subsection{Experimental Results}

\subsubsection{Specimen Failures}

All specimens were loaded to failure with exception of B1. In the case of B1, a machine malfunction occurred that prevented the loading from reaching failure levels. With the exception of B1 (as stated before) and B9 (specimen split) all specimens failed between the bending points as expected. The average load for the specimens from Bedford Reinforced Plastics Inc. (group 1) was $335.31 \mathrm{lbs}$. These broken specimens can be seen in Figure 4.8. The lowest average load, $57.31 \mathrm{lbs}$, was recorded for the specimens from the Creative Pultrusions Superdeck (group 2). These specimens are seen in Figure 4.9. For the five specimens from Bedford cut from the sheet (group 3) the average load was $312.31 \mathrm{lbs}$. Figure 4.10 shows the broken specimens of this type. The specimen in Figure 4.11 (B9) shows a delamination failure.

\subsubsection{Frequency vs. Amplitude}

The frequency vs. amplitude graphs show the same pattern as that displayed by the plots for the tension tests. Figures $4.12,4.13$, and 4.14 are the plots of frequency vs. amplitude for tests B2, B7, and C2, respectively. As can be seen, the graphs for each of these specimens from 
different manufacturers or of different architecture show very much the same pattern. This pattern does, however, begin to show itself early in the group 1 pieces (test B2, Figure 4.12). This will be seen more clearly in chapter 6 . Of particular interest on these graphs is the area bounded by $70 \mathrm{~dB}$ and $100 \mathrm{kHz}-400 \mathrm{kHz}$. This seems to be the area where signals appear only when the specimen is approaching failure. The frequency vs. amplitude plots for the remaining 12 tests can be seen in Appendix-D.

\subsubsection{Amplitude vs. Duration}

The amplitude vs. duration plots also show much the same pattern as the ones for the tension tests. These plots are also displayed for tests B2, B7, and C2 in Figures 4.15, 4.16, and 4.17 , respectively. As can be seen, the signals begin very concentrated near the origin, $(0$ $\mu$ seconds, $40 \mathrm{~dB}$ ). As the loading increases, the signals explode outward on the graph. The explosion grows quicker along the $\mathrm{y}$-axis (amplitude) as opposed to the x-axis (duration). This happens for all specimens regardless of manufacturer or architecture. Appendix-E is a grouping of the amplitude vs. duration plots for the other 12 specimens.

\subsubsection{EEnergy vs. Load}

The summation Energy vs. load plots for the bending specimens have a much more pronounced and consistent pattern compared to the same plots for the tension specimens. Figures 4.18, 4.19, and 4.20 show the $\Sigma$ energy vs. load plots for tests B2, B7, and C2. As can be seen, these plots display a very distinctive and strong pattern with a pronounced turning point. Staying consistent with the frequency vs. amplitude plots, the turning point occurs earlier for the Bedford deck specimens. The specimens from the Bedford plate section and Creative deck 
section have turning points at comparable locations. To see this pattern in all of the specimens, refer to Appendix-F, which contains the plots for the remaining 12 specimens.

\subsubsection{Load vs. Deflection}

Deflection for the bending specimens was measured as stated early. The deflection measured is the difference between the maximum deflection at mid-span and the deflection at the loading point. For our purposes, this deflection will be denoted as $\Delta_{\text {mid. }}$ This deflection can be seen in Figure 4.21. Figures 4.22, 4.23, and 4.24 are standard load-deflection curves for each of the types of specimens. The plots for the rest of the specimens are in Appendix-G. The fluctuation of both the load and deflection parameters is caused by the truncation of signals entering the LOCAN AT system. In order to find E for the specimens, the following equations were used.

$$
\begin{aligned}
& \Delta_{\max }=\frac{P a}{24 E I}\left(3 l^{2}-4 a^{2}\right) \\
& \Delta_{x}=\frac{P x}{6 E I}\left(3 l a-3 a^{2}-x^{2}\right)
\end{aligned}
$$

where,

$$
\begin{aligned}
& \mathrm{P}=\text { Loading point force }(1 / 2 \text { of the load from the load vs. deflection curve }) \\
& \mathrm{E}=\text { Young's modulus of the material } \\
& \mathrm{a}=\text { Distance from support to loading point }(2.67 \text { inches }) \\
& \mathrm{I}=\text { Length of specimen }(8 \text { inches })
\end{aligned}
$$


$\mathrm{x}=$ Distance to point where deflection is to be found

$\mathrm{I}=$ Moment of Inertia

From this, the following was used to find $\mathrm{E}$ for the specimens.

$$
\Delta_{\text {mid }}=\Delta_{\text {max }}-\Delta_{x}
$$

Inputting known values $(1=8, \mathrm{a}=\mathrm{x}=2.67)$ and simplifying leads to the following equation for E.

$$
E=28.34 \frac{m}{h^{3}}
$$

where,

$$
\begin{aligned}
& \mathrm{m}=\text { Slope of tangent to initial straight line section of the load vs. deflection curve } \\
& \mathrm{h}=\text { thickness of specimen }
\end{aligned}
$$

From this equation, the Young's Modulus for each type of specimen was calculated. These approximate values are $5.27 \times 10^{6} \mathrm{psi}$ for the Bedford deck (group 1) specimens, $1.01 \times 10^{6}$ psi for the Bedford plate pieces (group 3), and $1.93 \times 10^{6}$ psi for the specimens from the Creative deck (group 2). These can be seen in Table 4.1. The fluctuation of the load and deflection parameters causes these parameters to be slightly off and inconsistent. They do however give a ball park figure. It is also noted that shear deformation influences these modulus values. The $\mathrm{L} / \mathrm{d}$ ratios chosen were in an attempt to minimize this effect, but otherwise, the shear effect was not accounted for. 


\begin{tabular}{|c|c|c|c|c|}
\hline \multicolumn{5}{|c|}{ Group 1 } \\
\hline specimen & load & slope (m) & height (h) & E \\
\hline & (lbs) & (Ibs/in) & (in) & $\left(10^{6} \mathrm{psi}\right)$ \\
\hline B1 & - & 2136 & 0.25 & 3.87 \\
\hline B2 & 347.31 & 3106 & 0.25 & 5.63 \\
\hline B3 & 315.31 & 3375 & 0.25 & 6.12 \\
\hline B4 & 345.31 & 3025 & 0.25 & 5.49 \\
\hline B5 & 333.31 & 2897 & 0.25 & 5.25 \\
\hline
\end{tabular}

\begin{tabular}{l|l|l|l|l} 
Ave. & 335.31 & 2907.8 & 0.25 & $\mathbf{5 . 2 7}$ \\
\end{tabular}

\begin{tabular}{|c|c|c|c|c|}
\hline \hline \multicolumn{5}{|c|}{ Group 2 } \\
\hline specimen & load & slope (m) & height (h) & E \\
\hline & (lbs) & (Ibs/in) & (in) & $\left(10^{6} \mathrm{psi}\right)$ \\
\hline C1 & 55.31 & 458 & 0.2 & 1.62 \\
\hline C2 & 58.31 & 539 & 0.2 & 1.91 \\
\hline C3 & 69.31 & 754 & 0.2 & 2.67 \\
\hline C4 & 54.31 & 416 & 0.2 & 1.47 \\
\hline C5 & 52.31 & 560 & 0.2 & 1.98 \\
\hline
\end{tabular}
Ave.
\begin{tabular}{l|l}
57.91 & 545.4 \\
\hline
\end{tabular}
0.2
1.93

\begin{tabular}{|c|c|c|c|c|}
\hline \hline \multicolumn{5}{|c|}{ Group 3 } \\
\hline specimen & load & slope (m) & height (h) & E \\
\hline & (lbs) & (Ibs/in) & (in) & $\left(10^{6} \mathrm{psi}\right)$ \\
\hline B6 & 270.31 & 5329 & 0.5 & 1.21 \\
\hline B7 & 333.31 & 3182 & 0.5 & 0.72 \\
\hline B8 & 314.31 & 5336 & 0.5 & 1.21 \\
\hline B9 & 345.31 & 3988 & 0.5 & 0.90 \\
\hline B10 & 298.31 & - & 0.5 & \\
\hline
\end{tabular}

\begin{tabular}{l|l|l|l|l} 
Ave. & 312.31 & 4458.75 & 0.5 & 1.01 \\
\hline
\end{tabular}

\begin{tabular}{|c|c|}
\hline width (in) & 0.5 \\
\hline support span (in) & 8 \\
\hline load span (in) & 2.67 \\
\hline
\end{tabular}

Table 4.1 Specimen Dimensions and Young's Modulus 


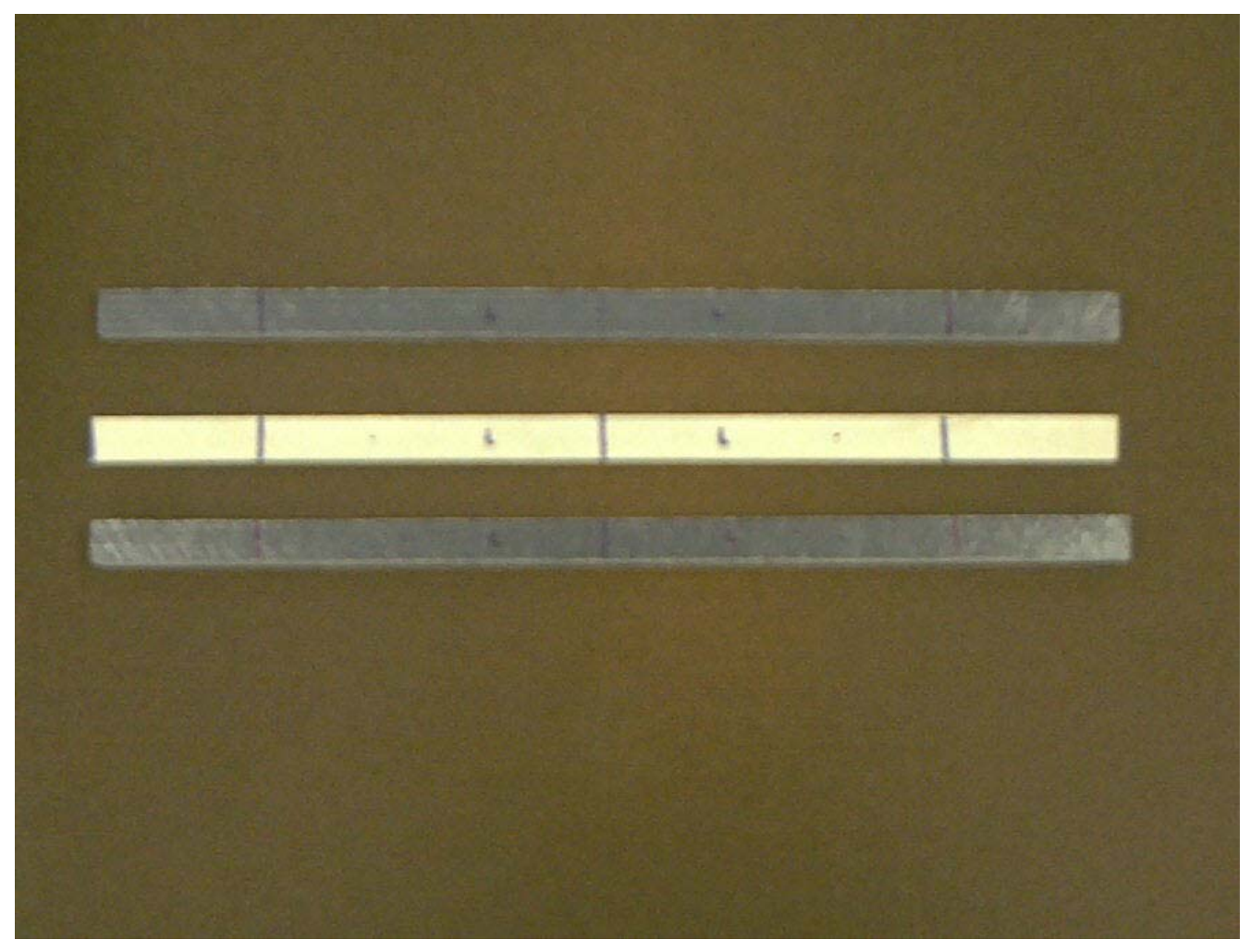

Figure 4.1 Unbroken Bending Specimens (group 1-top, group 2-middle, and group 3-bottom) 


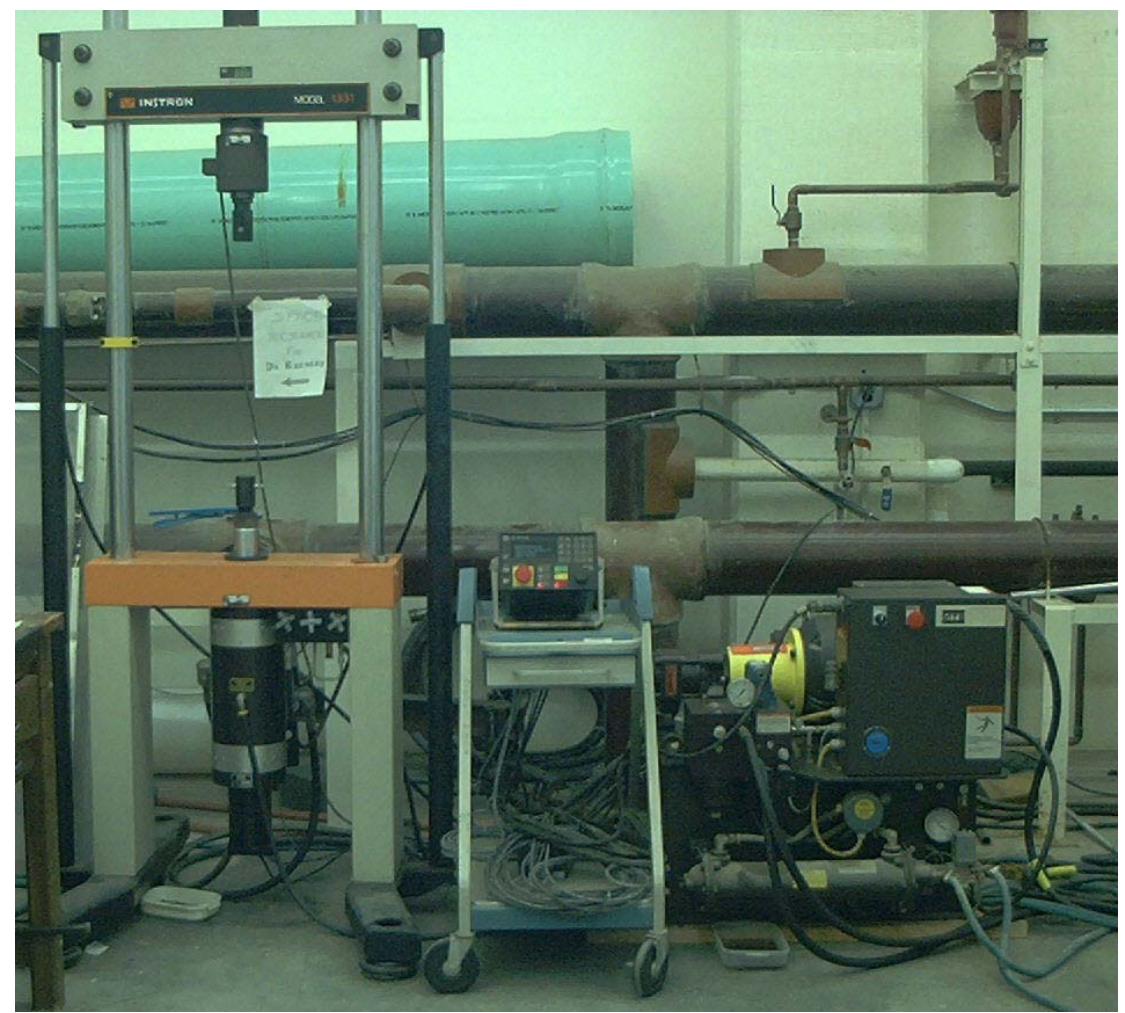

Figure 4.2 INSTRON Loading Frame, Actuator, Controller, and Hydraulic Power Supply

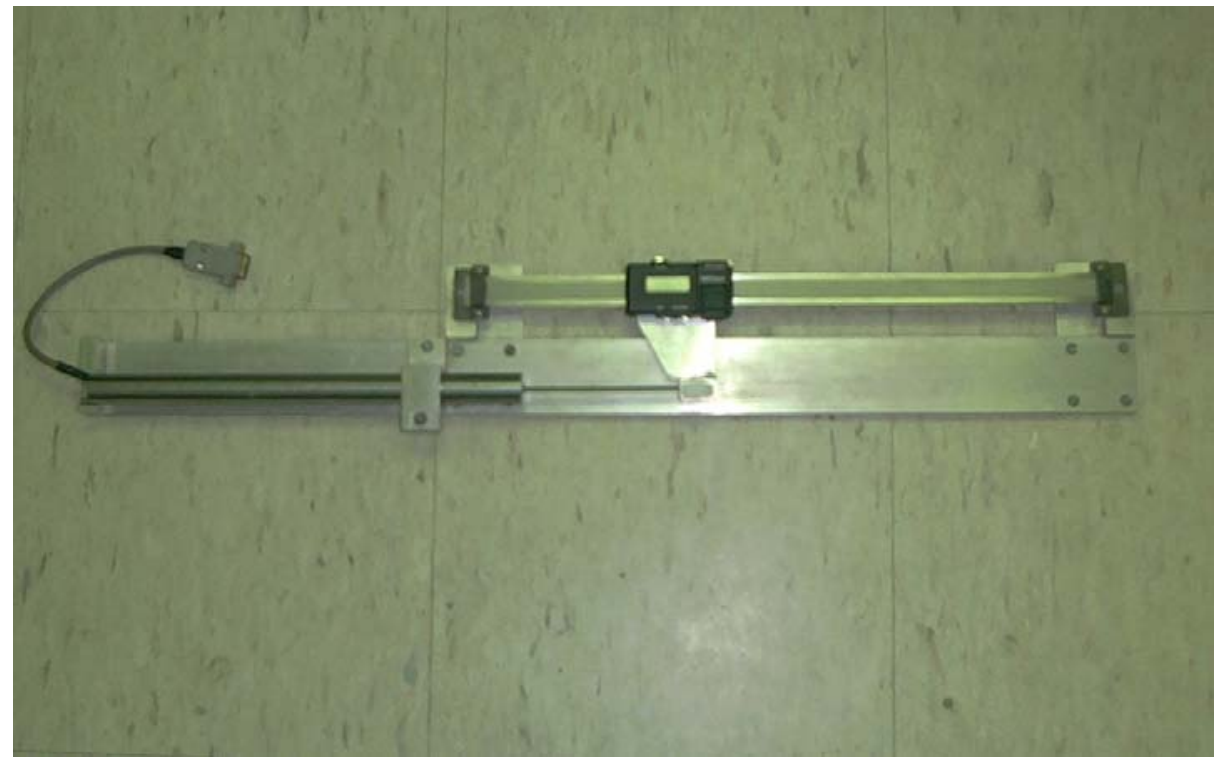

Figure 4.3 LVDT Calibration Tool 


\section{Calibration Curve: Schaevitz 2000 HR-DC with LOCAN AT connected}

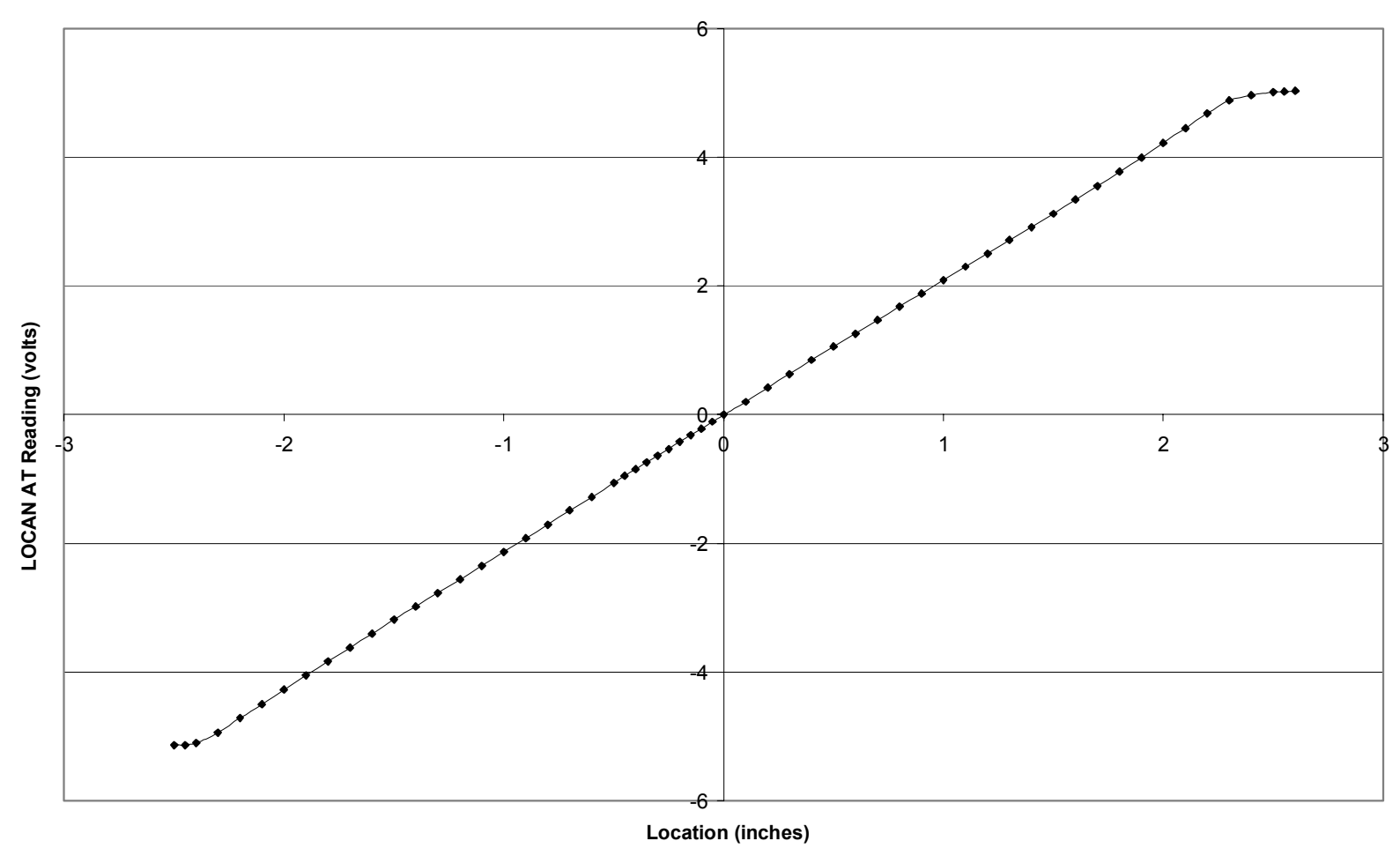

Figure 4.4 LVDT Calibration Curve 


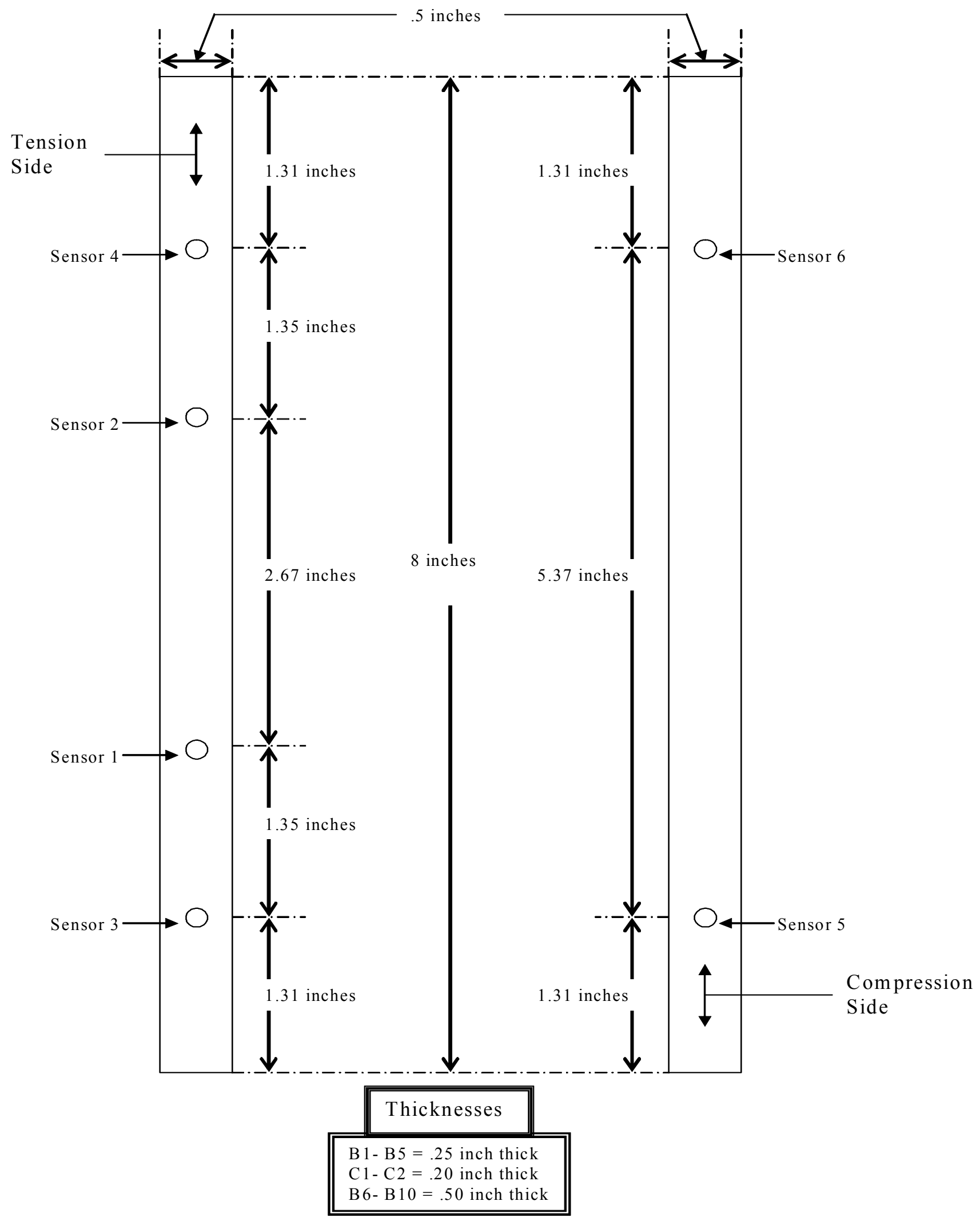

Figure 4.5 Specimen and Sensor Layout 


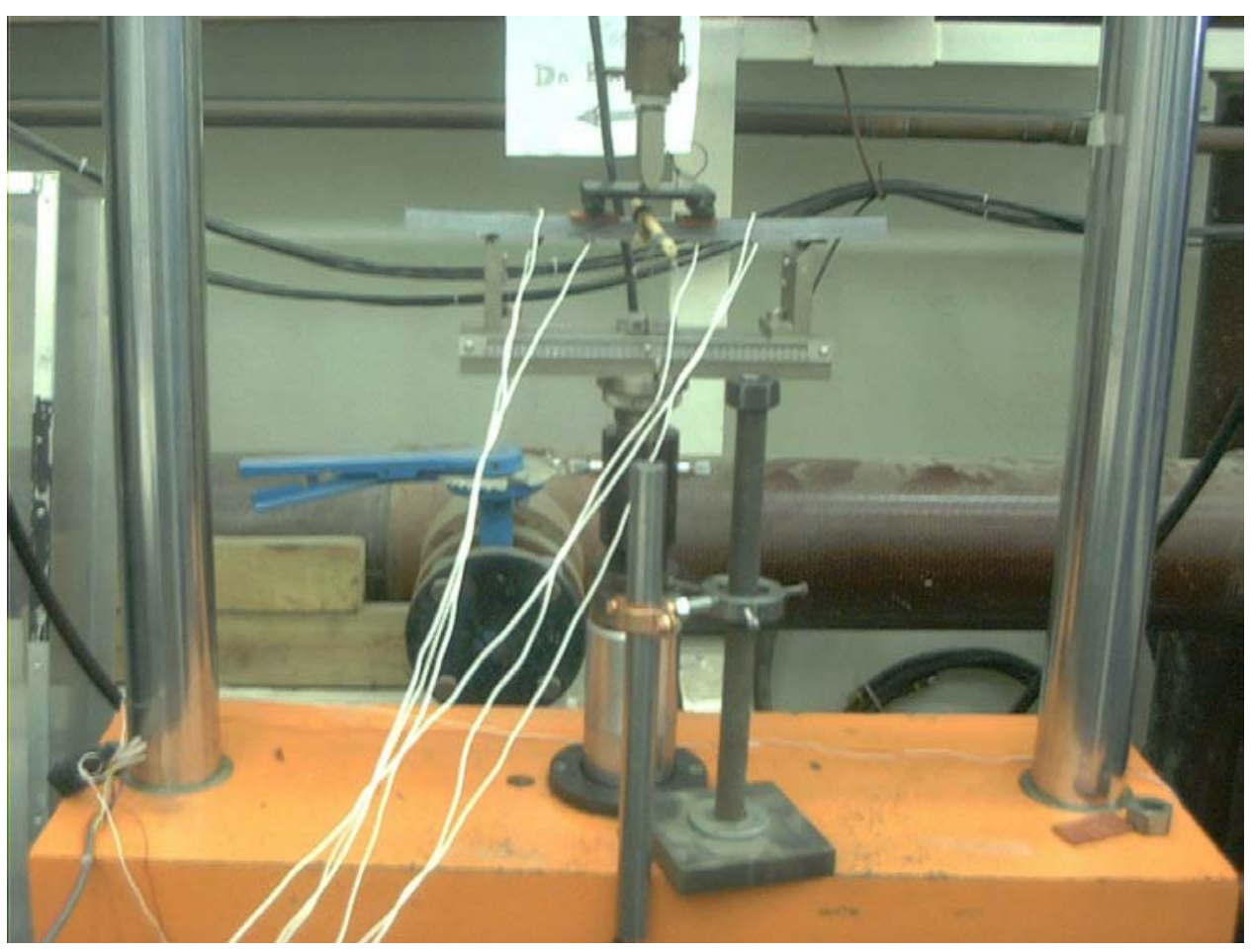

Figure 4.6 Unbent Specimen Prepared to be Loaded (B10)

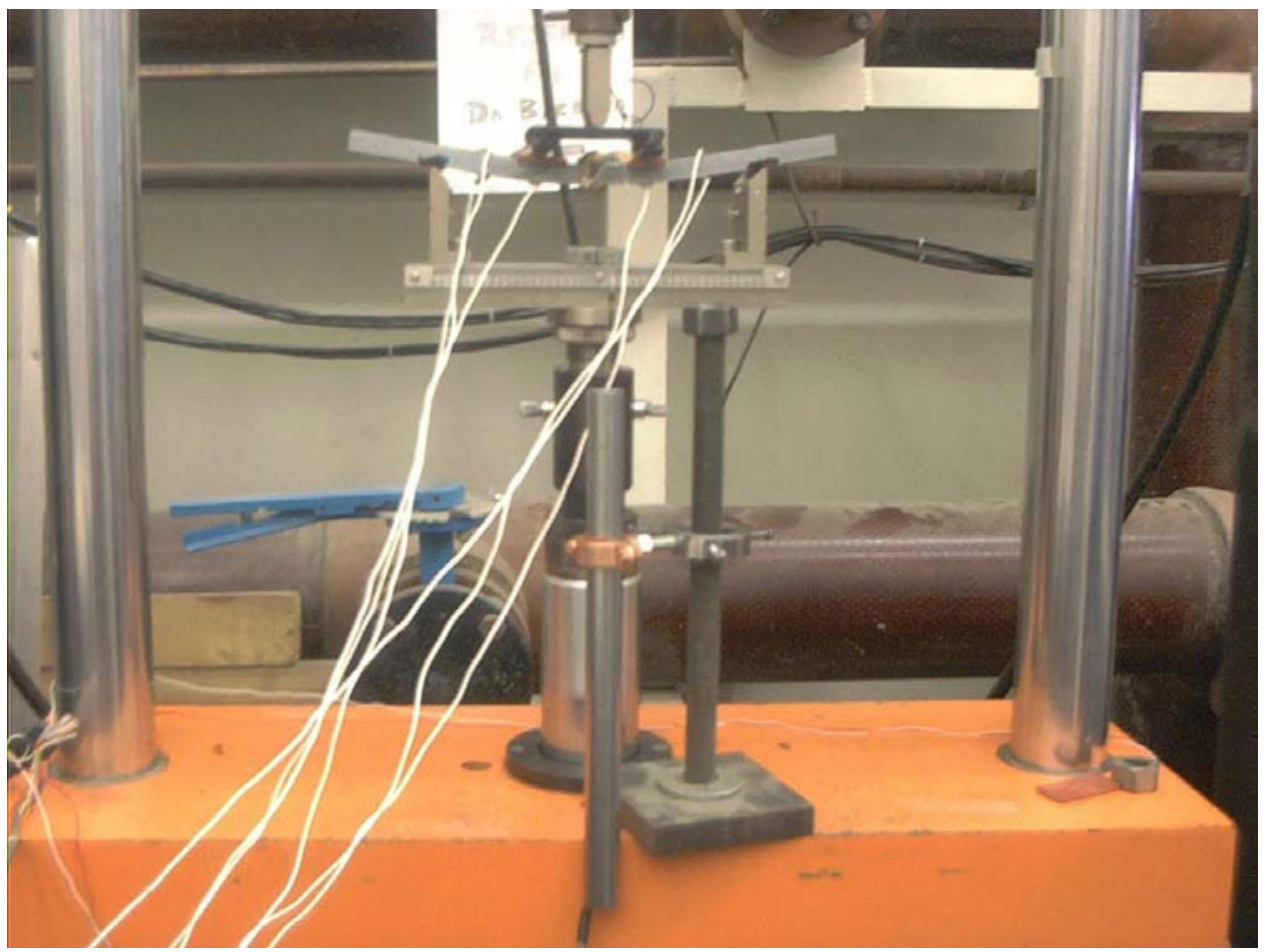

Figure 4.7 Bent Specimen Immediately Before Failure (B10) 


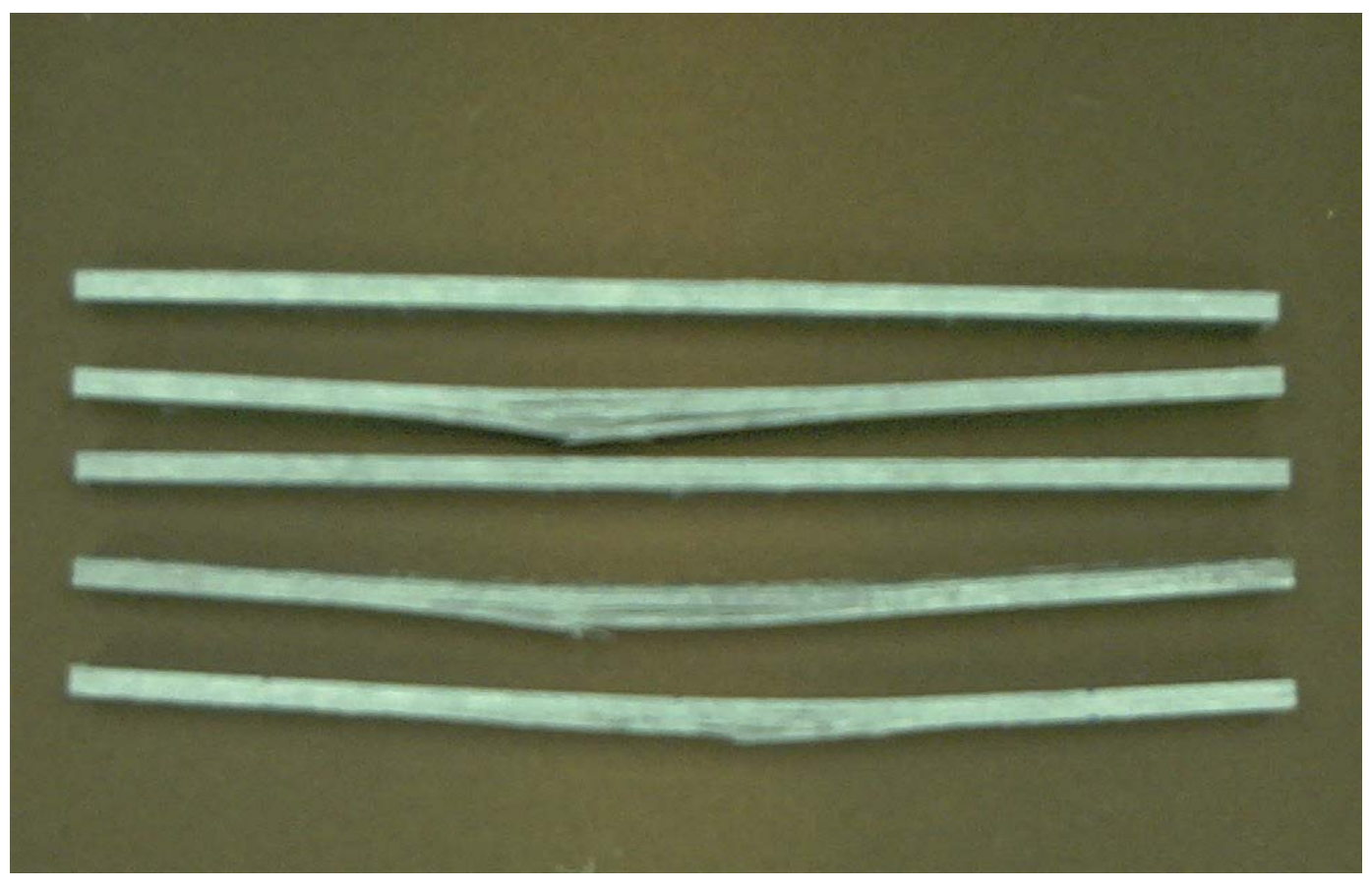

Figure 4.8 Broken Specimens, Group 1 (tests B1-B5)

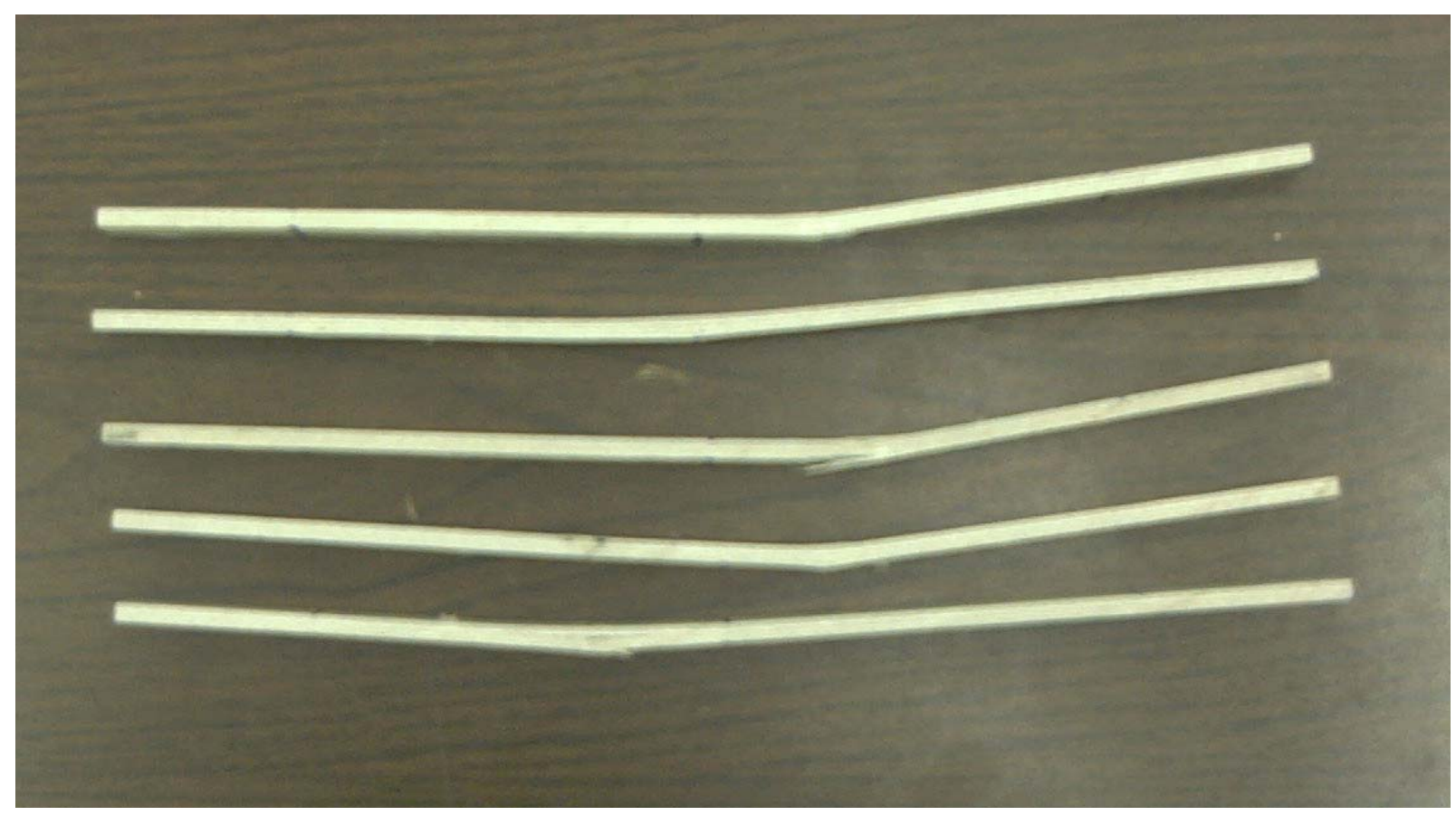

Figure 4.9 Broken Specimens, Group 2 (tests C1-C5) 


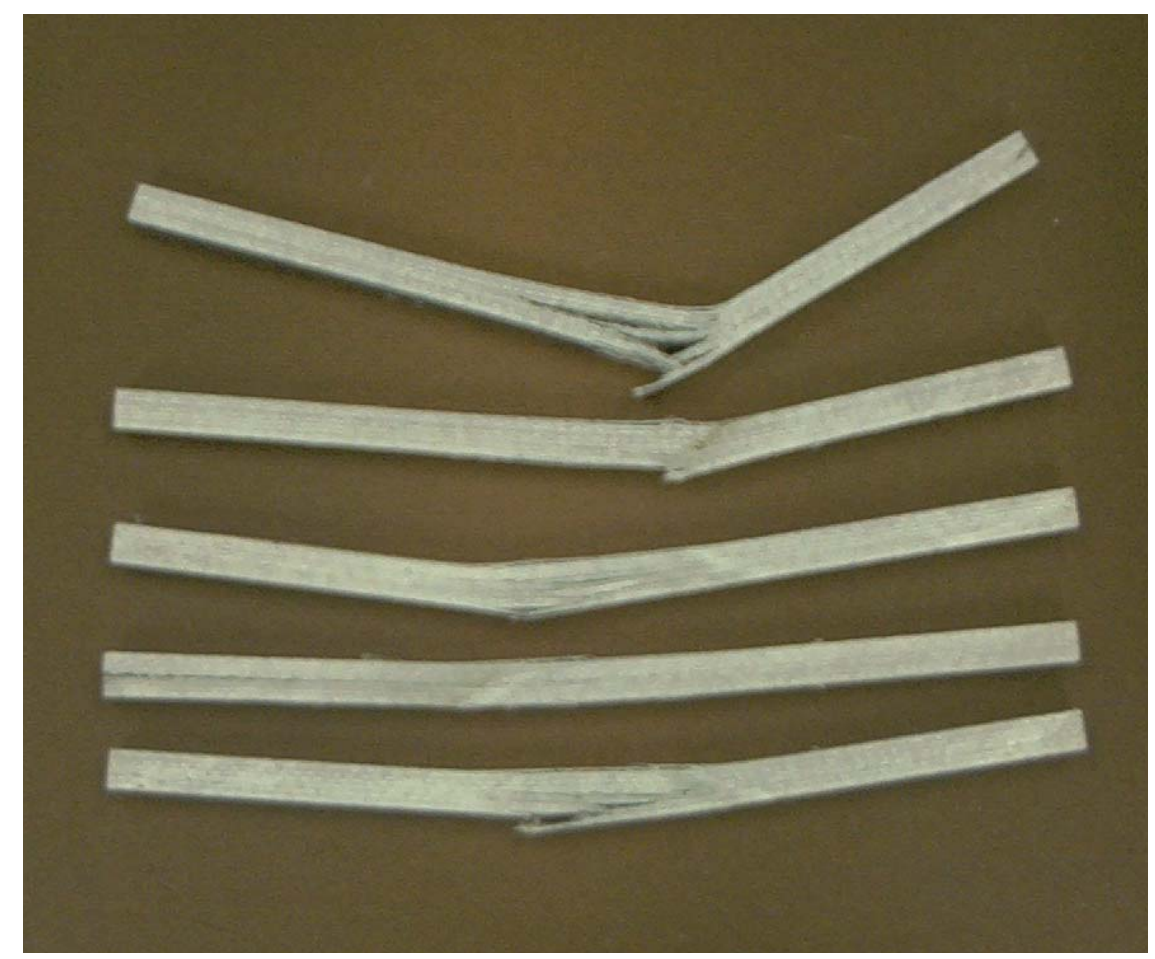

Figure 4.10 Broken Specimens, Group 3 (tests B6-B10)

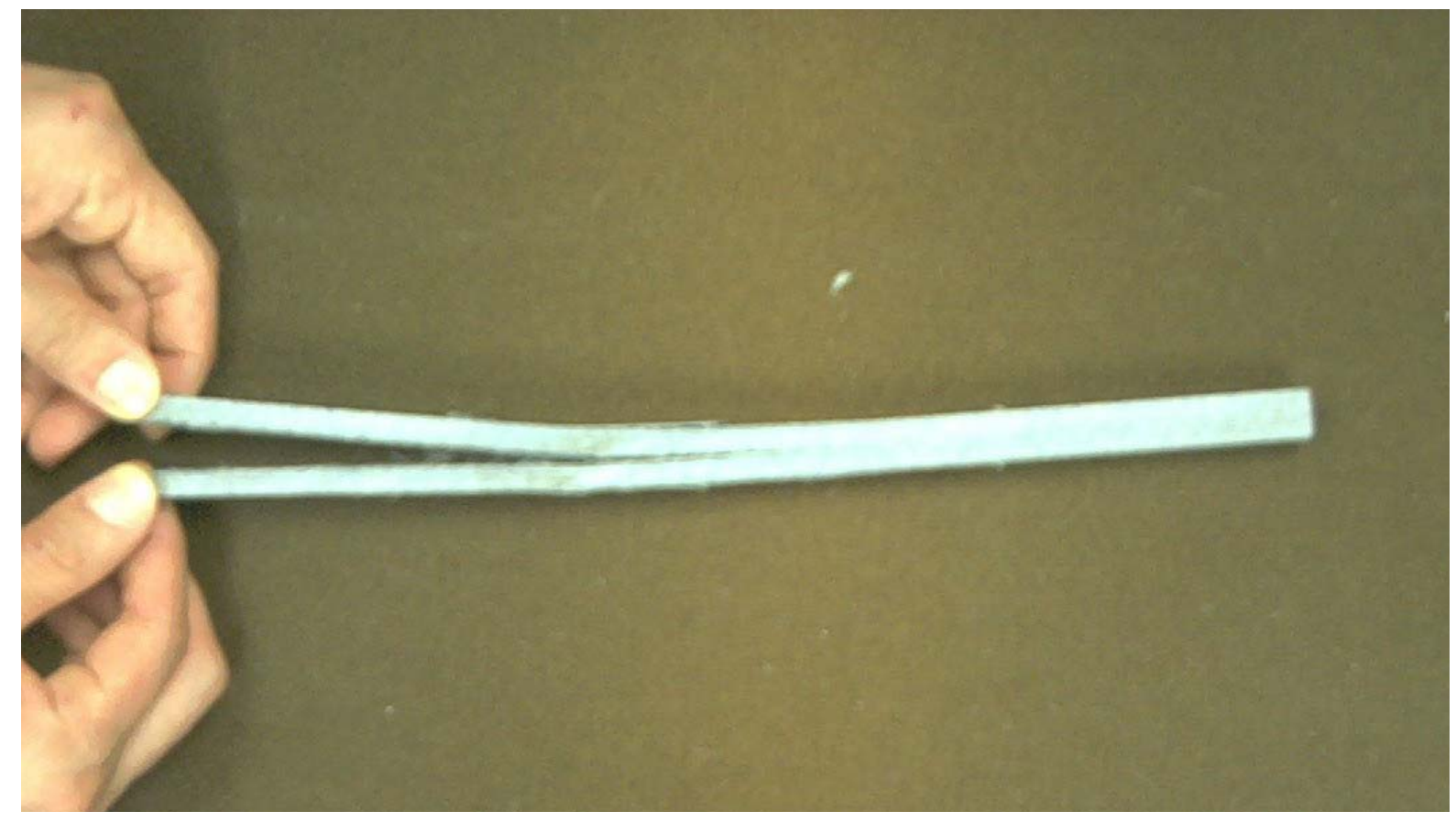

Figure 4.11 Delaminated Specimen (B9) 
Test B2, ch 182:Frequency vs Amplitude (1st quarter)

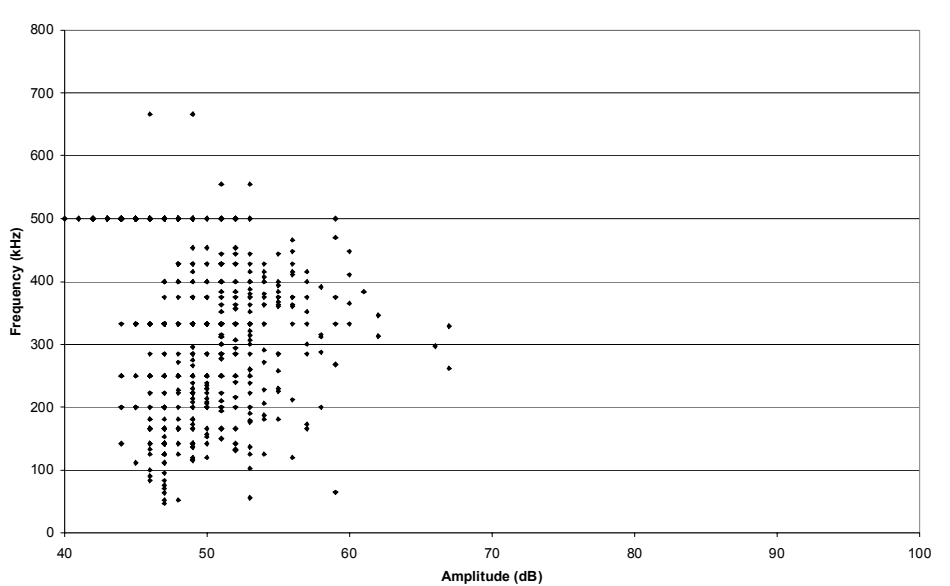

Test B2, ch 1\&2: Frequency vs Amplitude (2nd quarter)

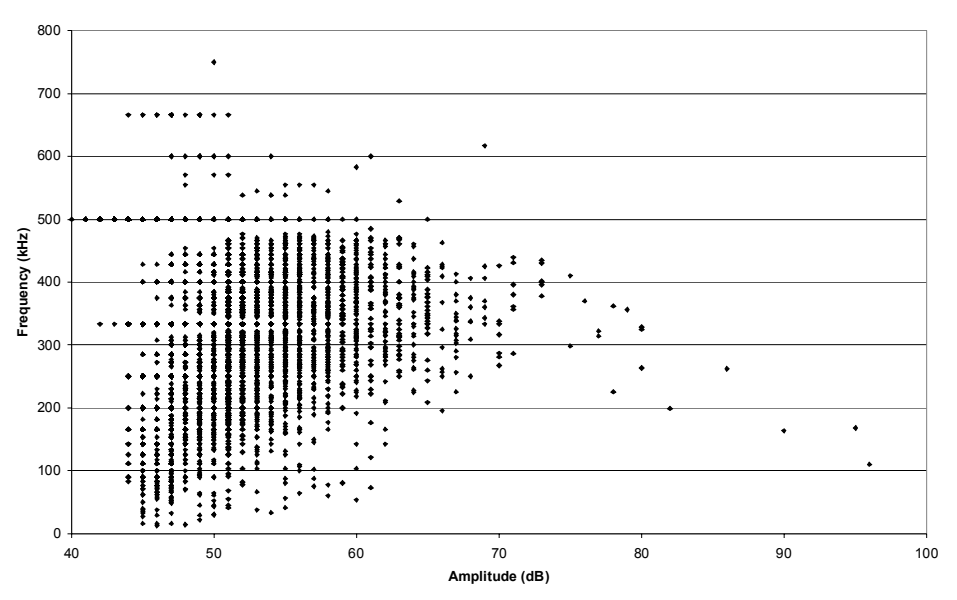

Test B2, ch 182: Frequency vs Amplitude (3rd quarter)

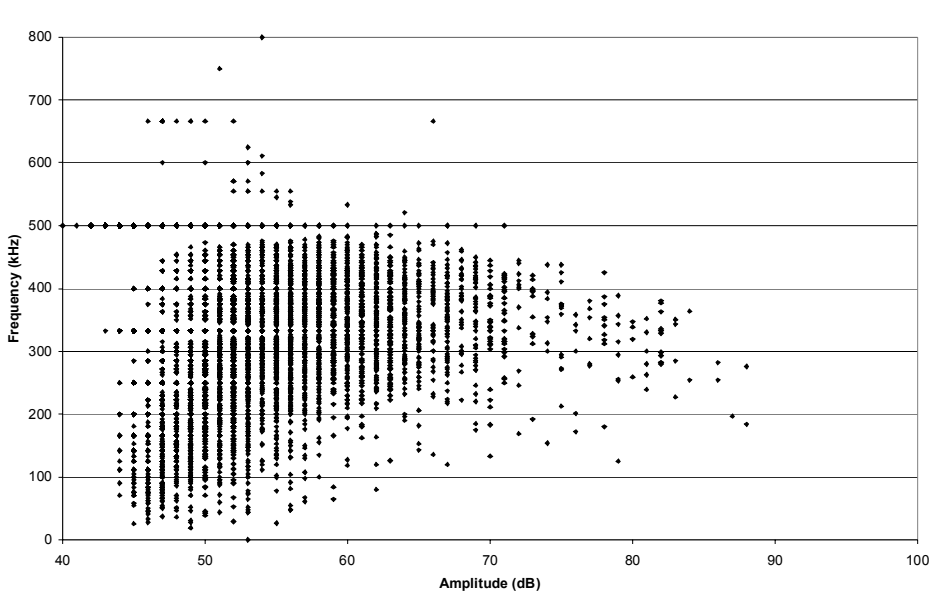

Test B2, ch 182:Frequency vs Amplitude (4th quarter)

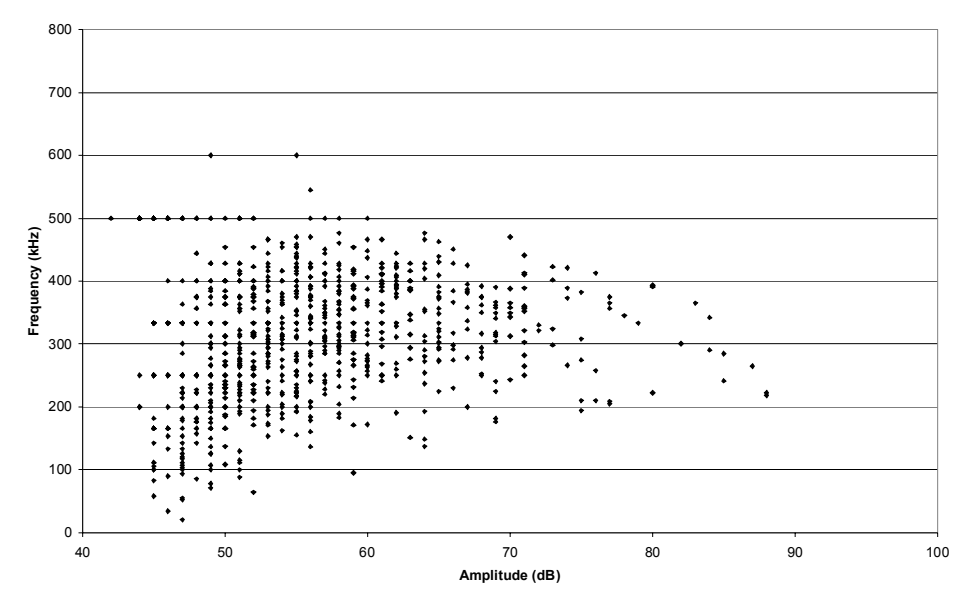

Figure 4.12 Frequency vs. Amplitude (test B2) 
Test B7, ch 182: Frequency vs Amplitude (1st quarter)

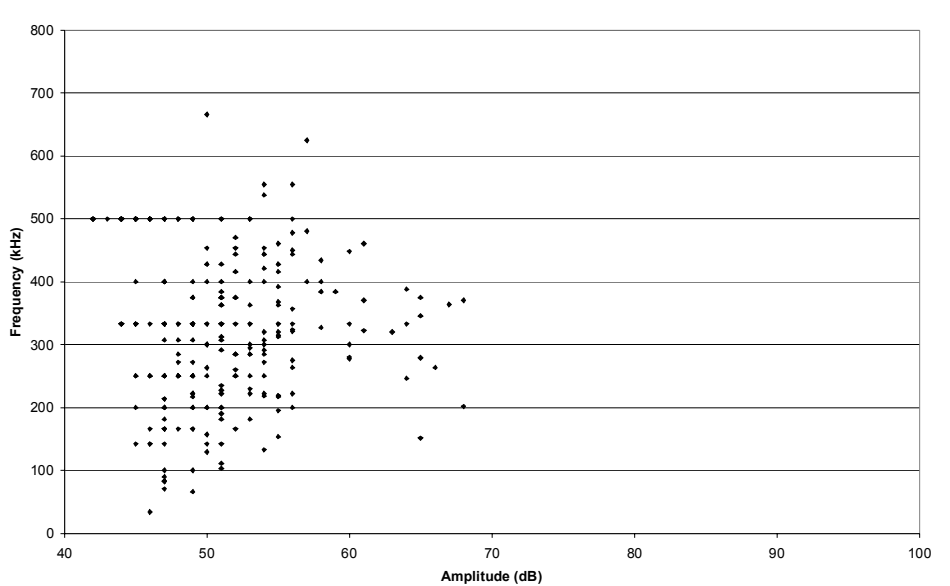

Test B7, ch 182: Frequency vs Amplitude (2nd quarter)

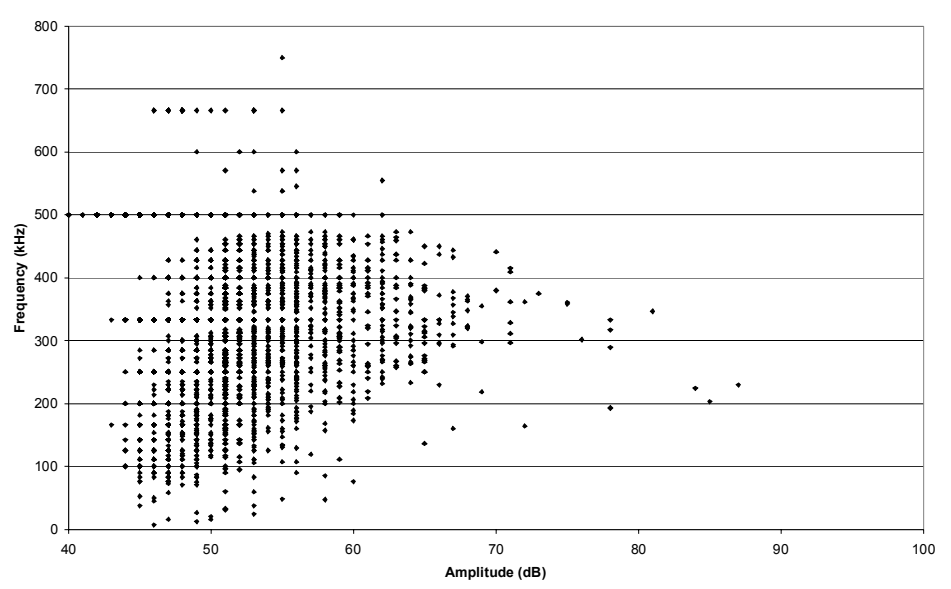

Test B7, ch 182: Frequency vs Amplitude (3rd quarter)

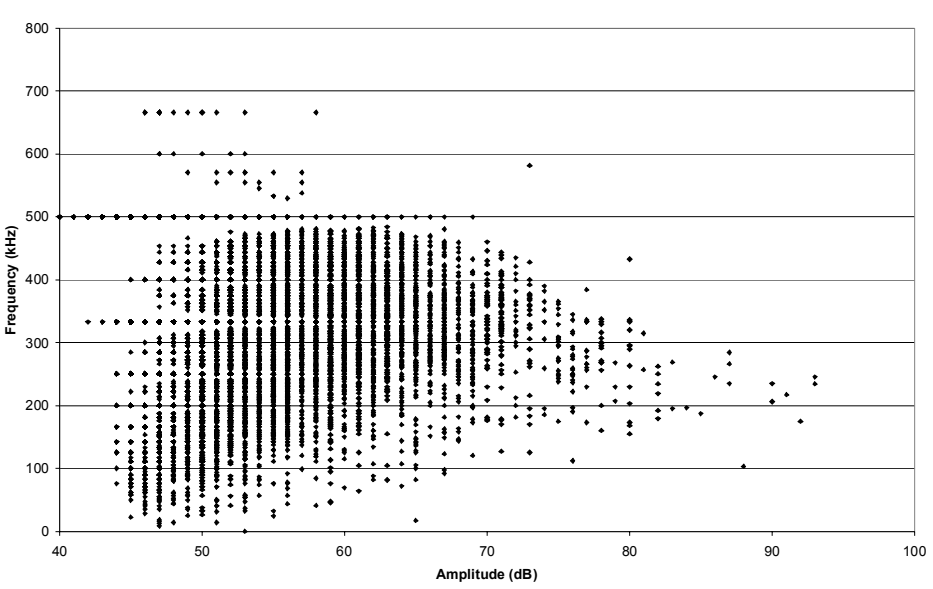

Test B7, ch 182: Frequency vs Amplitude (4th quarter)

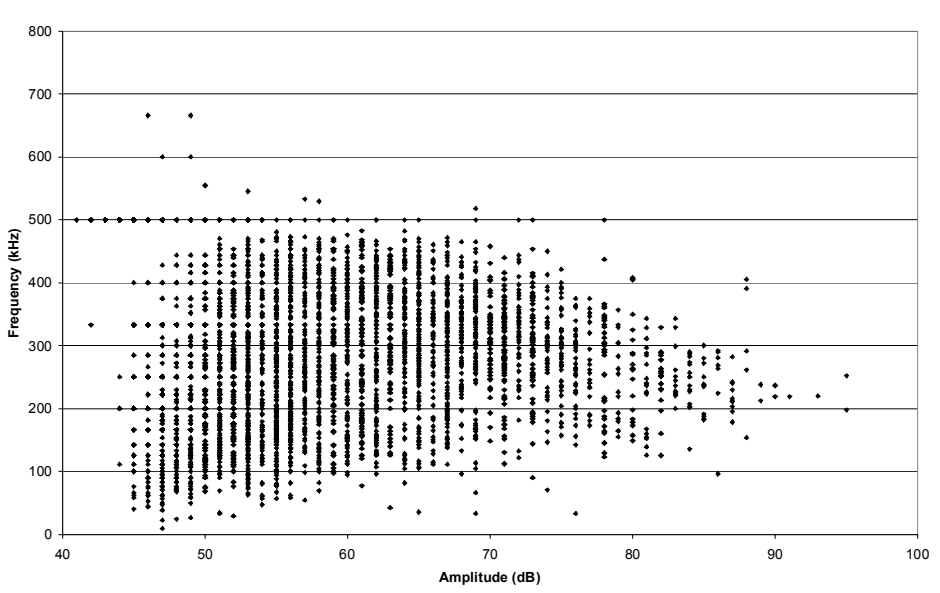

Figure 4.13 Frequency vs. Amplitude (test B7) 
Test C2, ch 122: Frequency vs Amplitude (1st quarter)

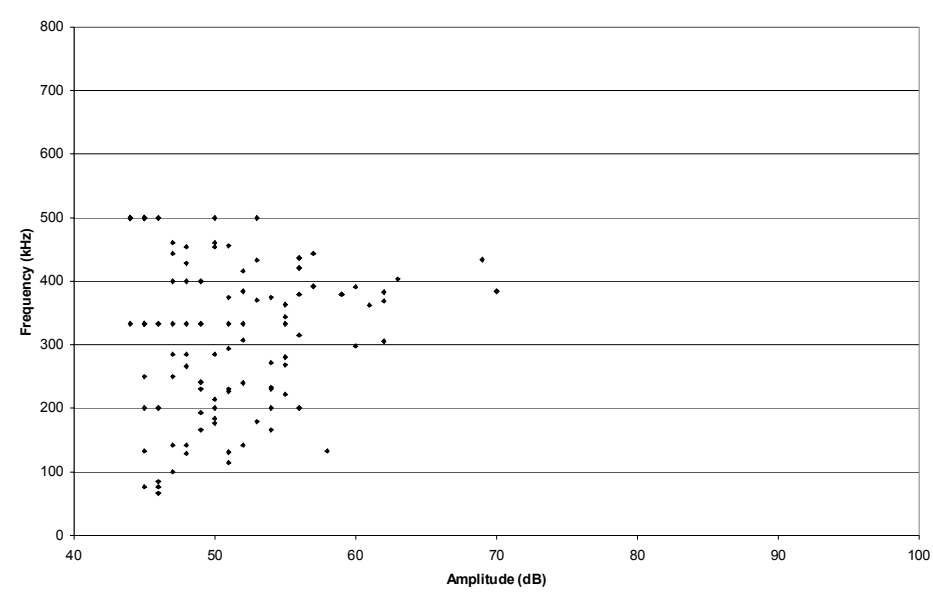

Test C2, ch 1\&2: Frequency vs Amplitude (2nd quarter)

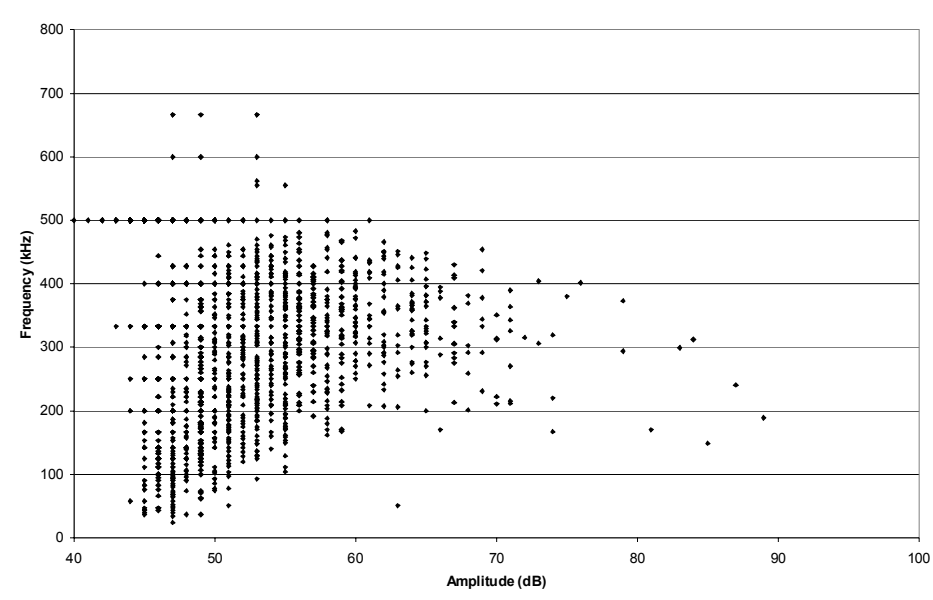

Test C2, ch 182: Frequency vs Amplitude (3rd quarter)

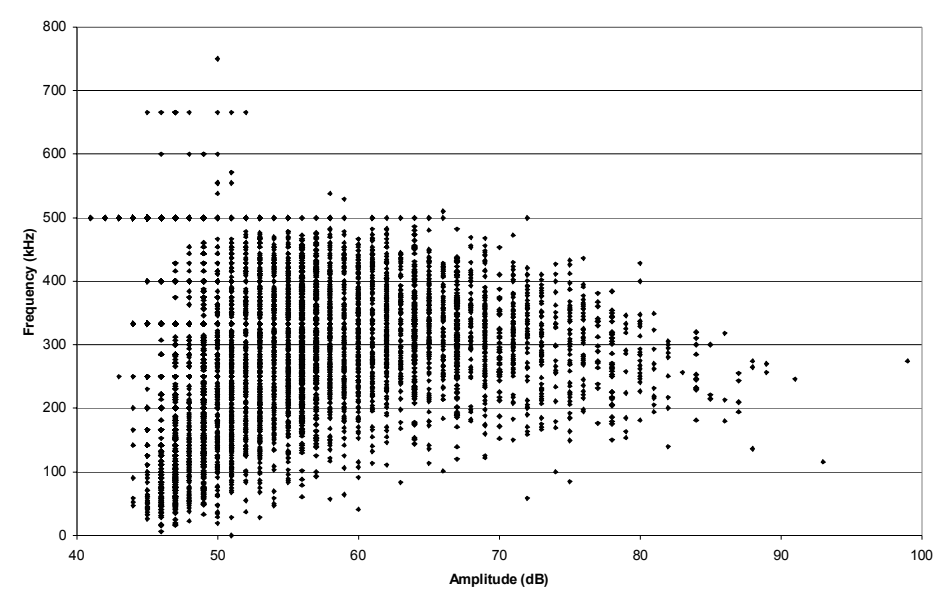

Test C2, ch 182: Frequency vs Amplitude (4th quarter)

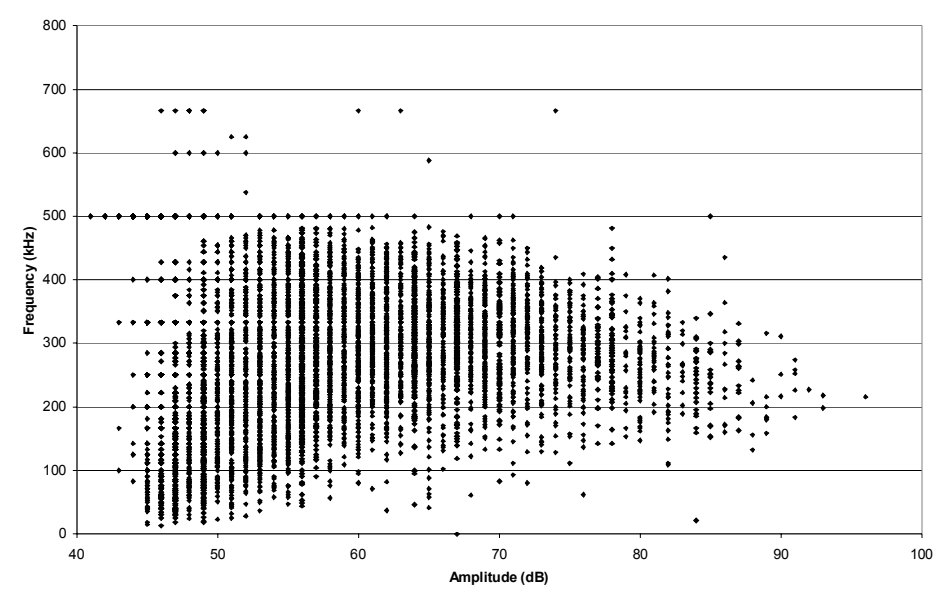

Figure 4.14 Frequency vs. Amplitude (test C2) 
Test B2, ch 1\&2: Amplitude vs Duration (1st quarter)

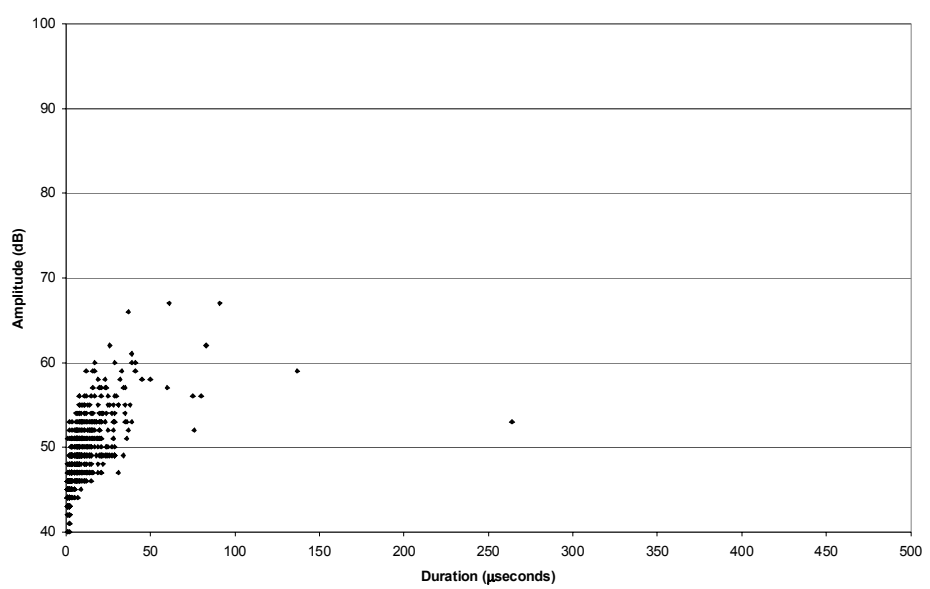

Test B2, ch 1\&2: Amplitude vs Duration (2nd quarter)

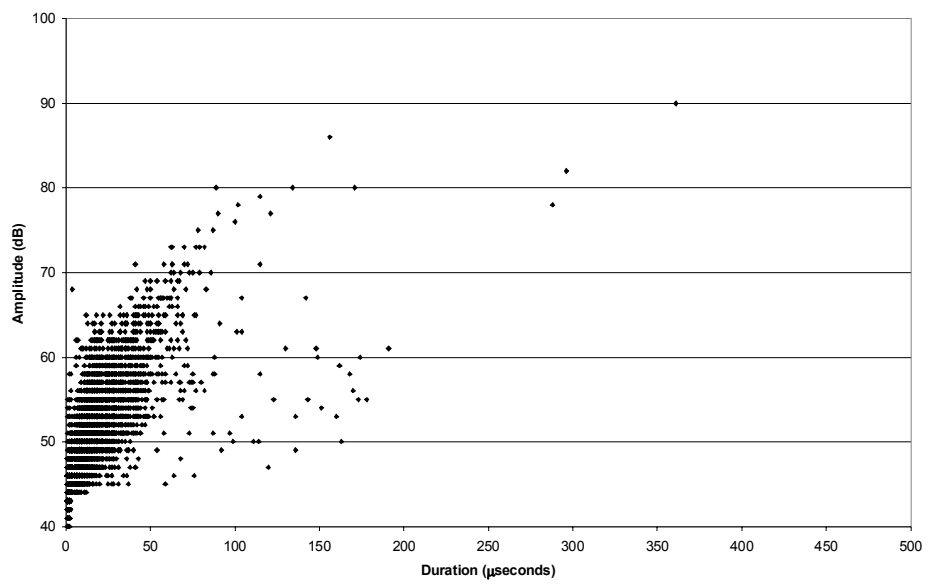

Test B2, ch 182: Amplitude vs Duration (3rd quarter)

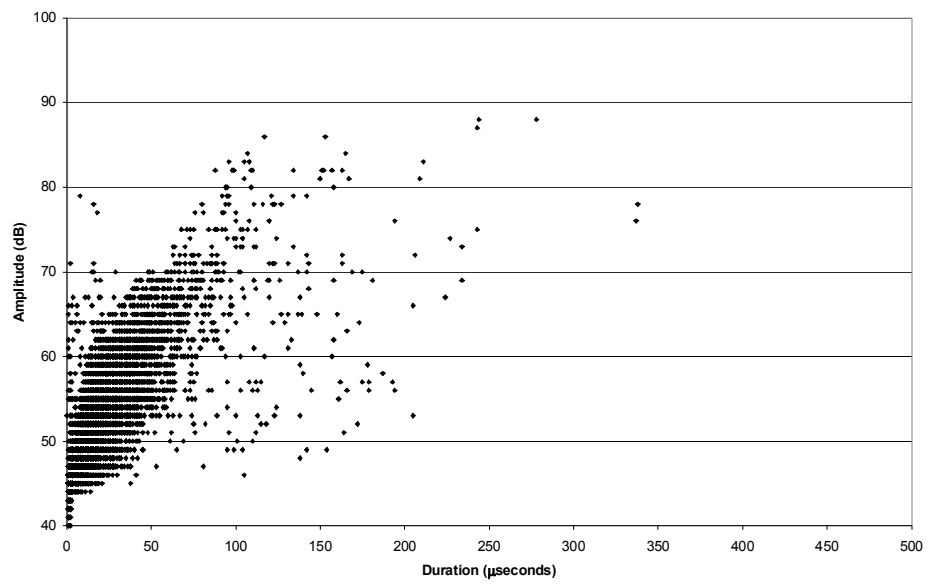

Test B2, ch 1\&2: Amplitude vs Duration (4th quarter)

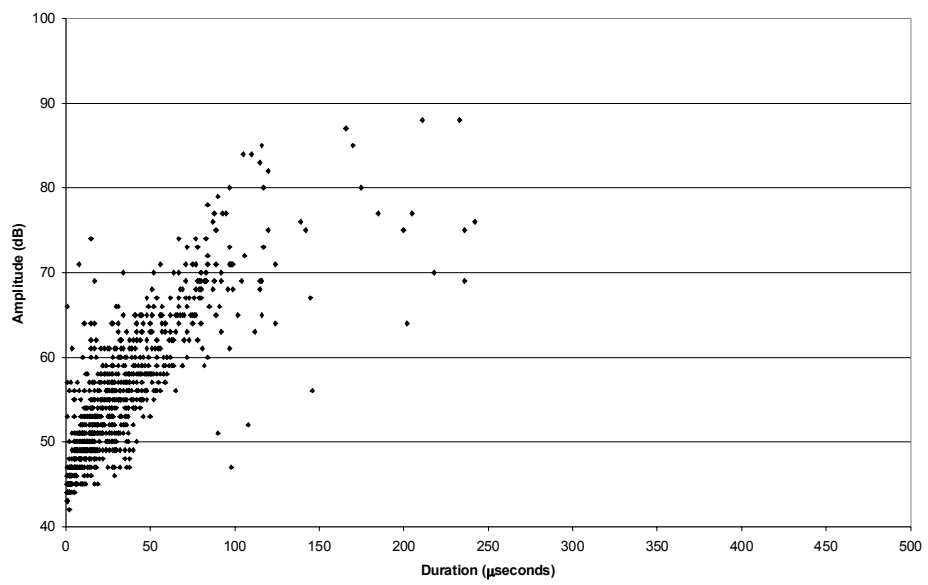

Figure 4.15 Amplitude vs. Duration (test B2)

69 
Test B7, ch 1\&2: Amplitude vs Duration (1st quarter)

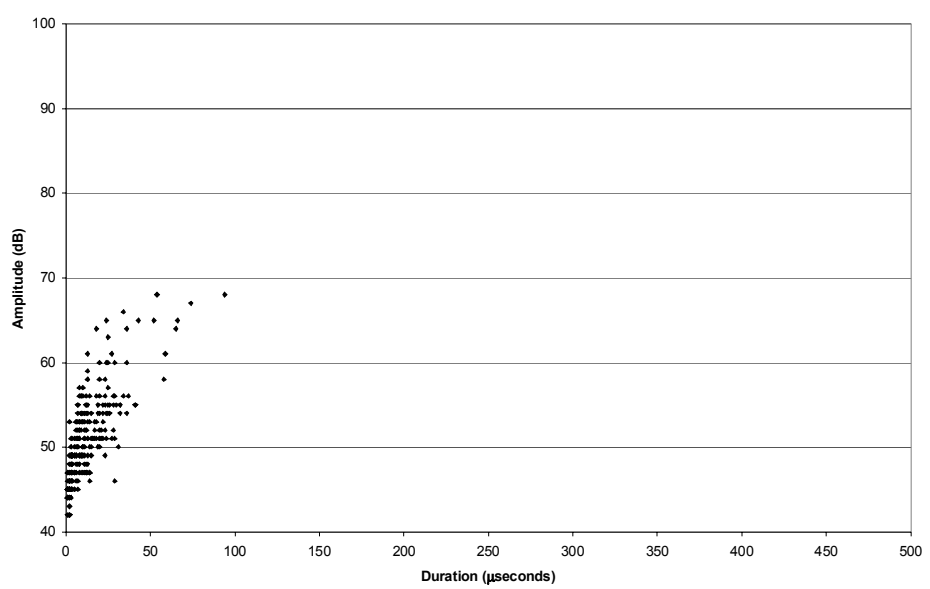

Test B7, ch 1\&2: Amplitude vs Duration (2nd quarter)

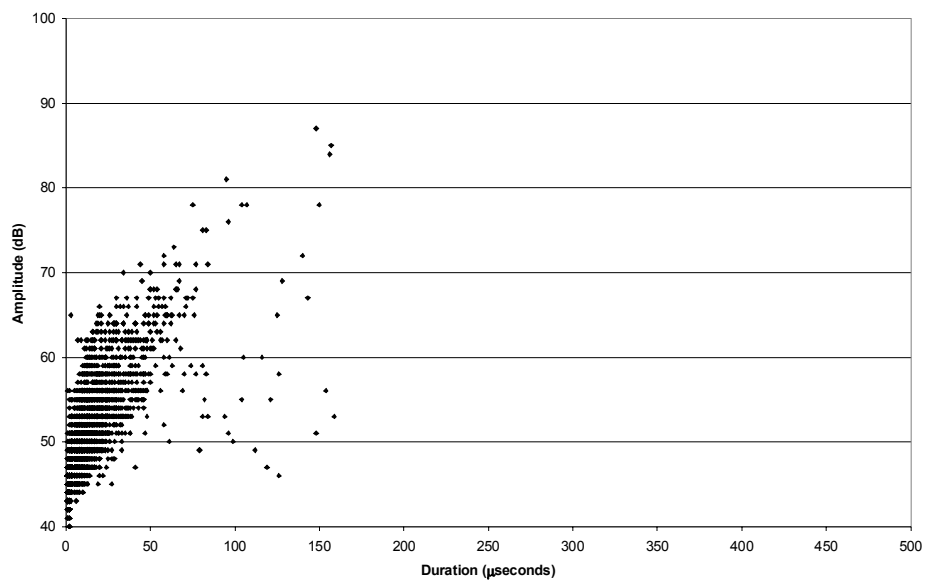

Test B7, ch 1\&2: Amplitude vs Duration (3rd quarter)

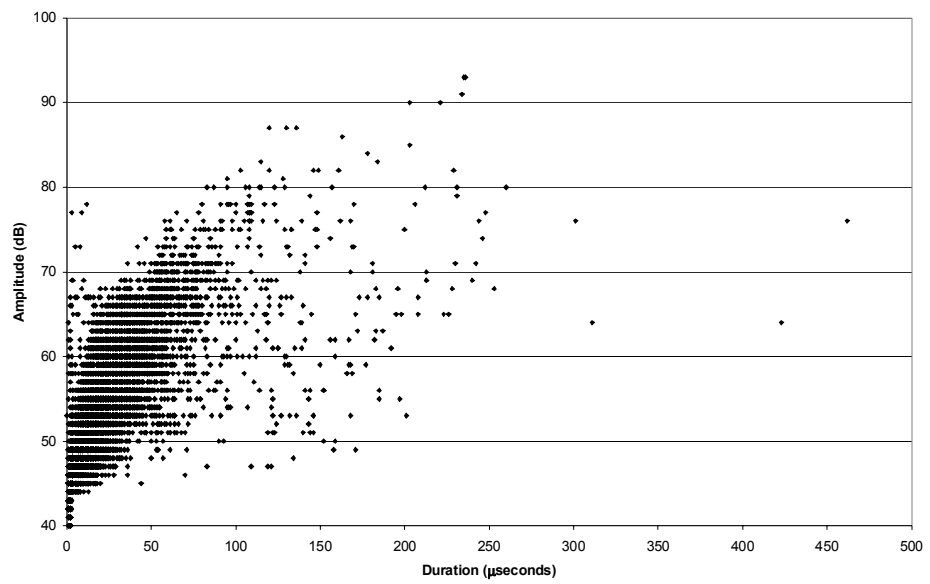

Test B7, ch 1\&2: Amplitude vs Duration (4th quarter)

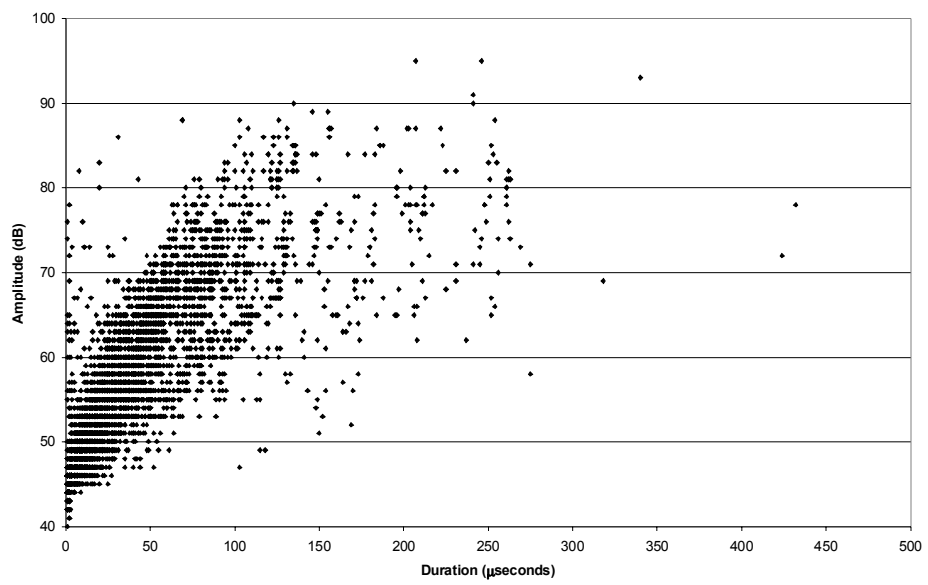

Figure 4.16 Amplitude vs. Duration (test B7)

70 
Test C2, ch 182: Amplitude vs Duration (1st quarter)

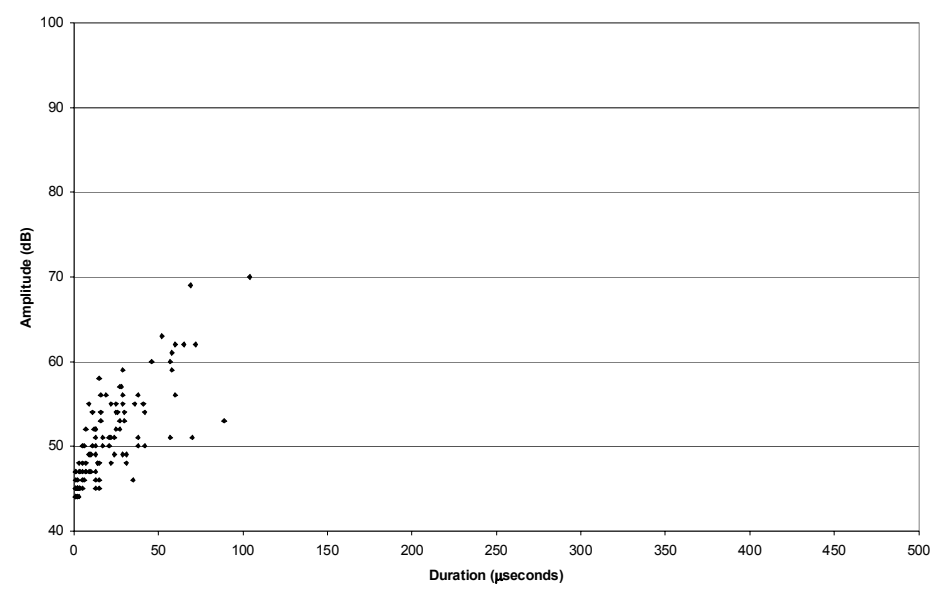

Test C2, ch 182: Amplitude vs Duration (2nd quarter)

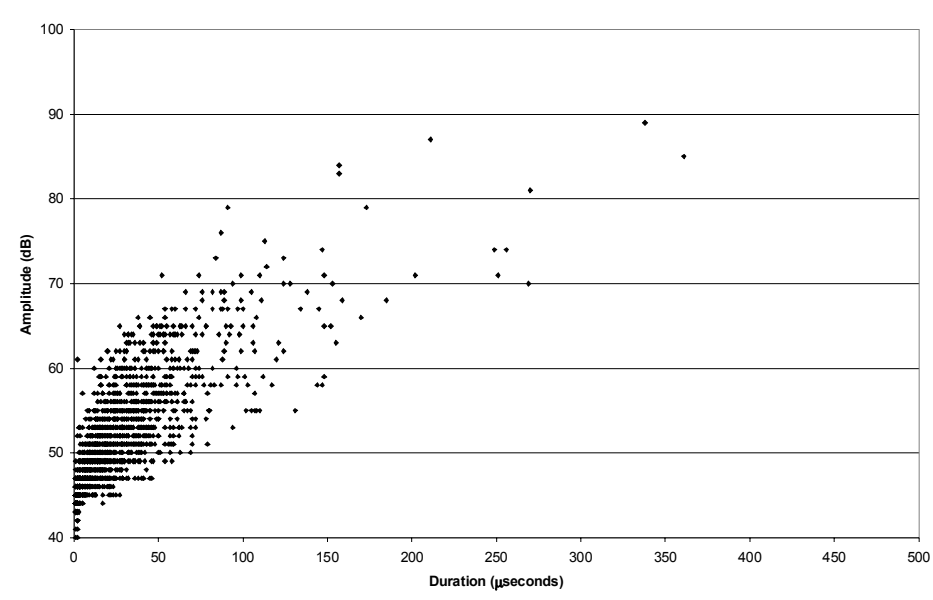

Test C2, ch 1\&2: Amplitude vs Duration (3rd quarter)

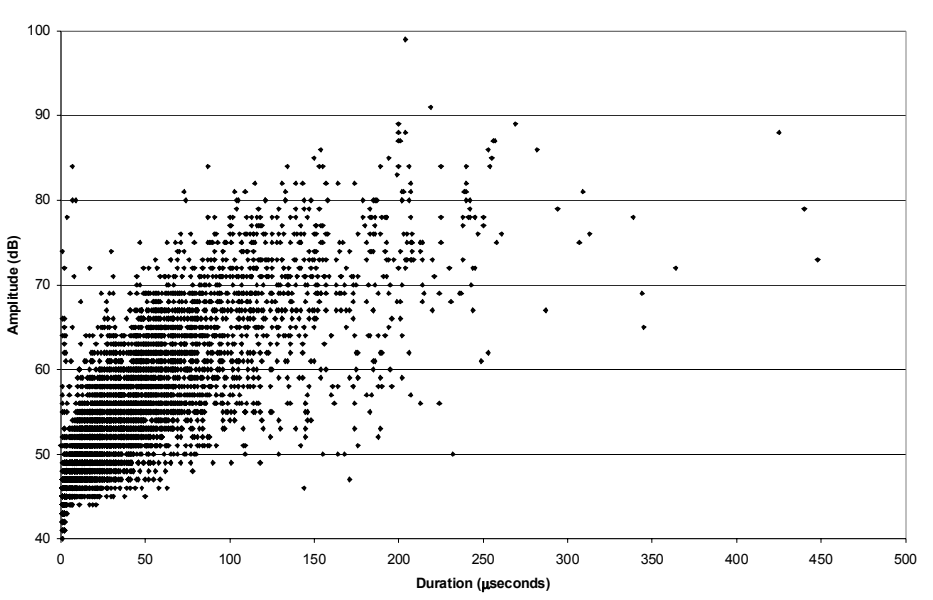

Test C2, ch 182: Amplitude vs Duration (4th quarter)

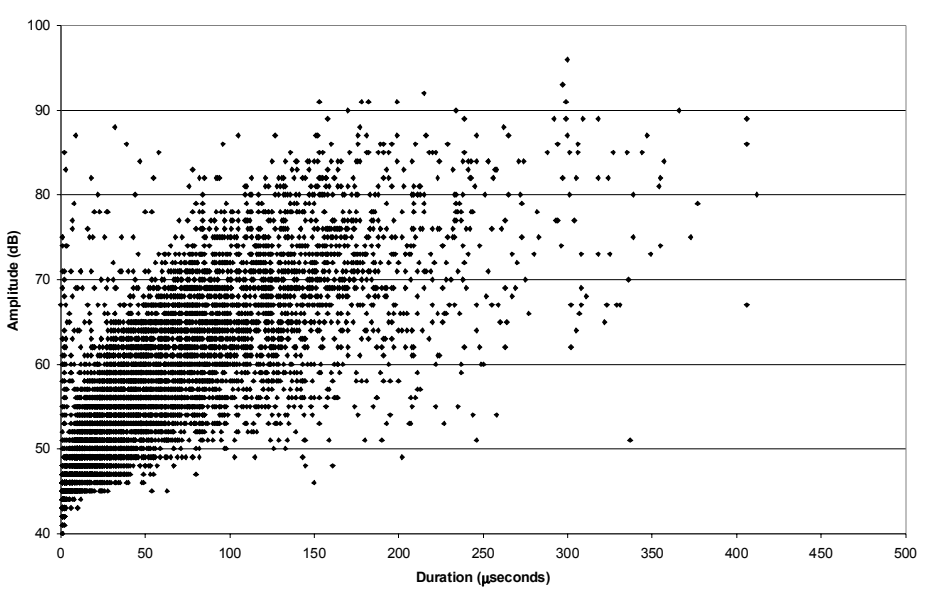

Figure 4.17 Amplitude vs. Duration (test C2) 
Test B2, ch 1\&2: Eenergy vs Load

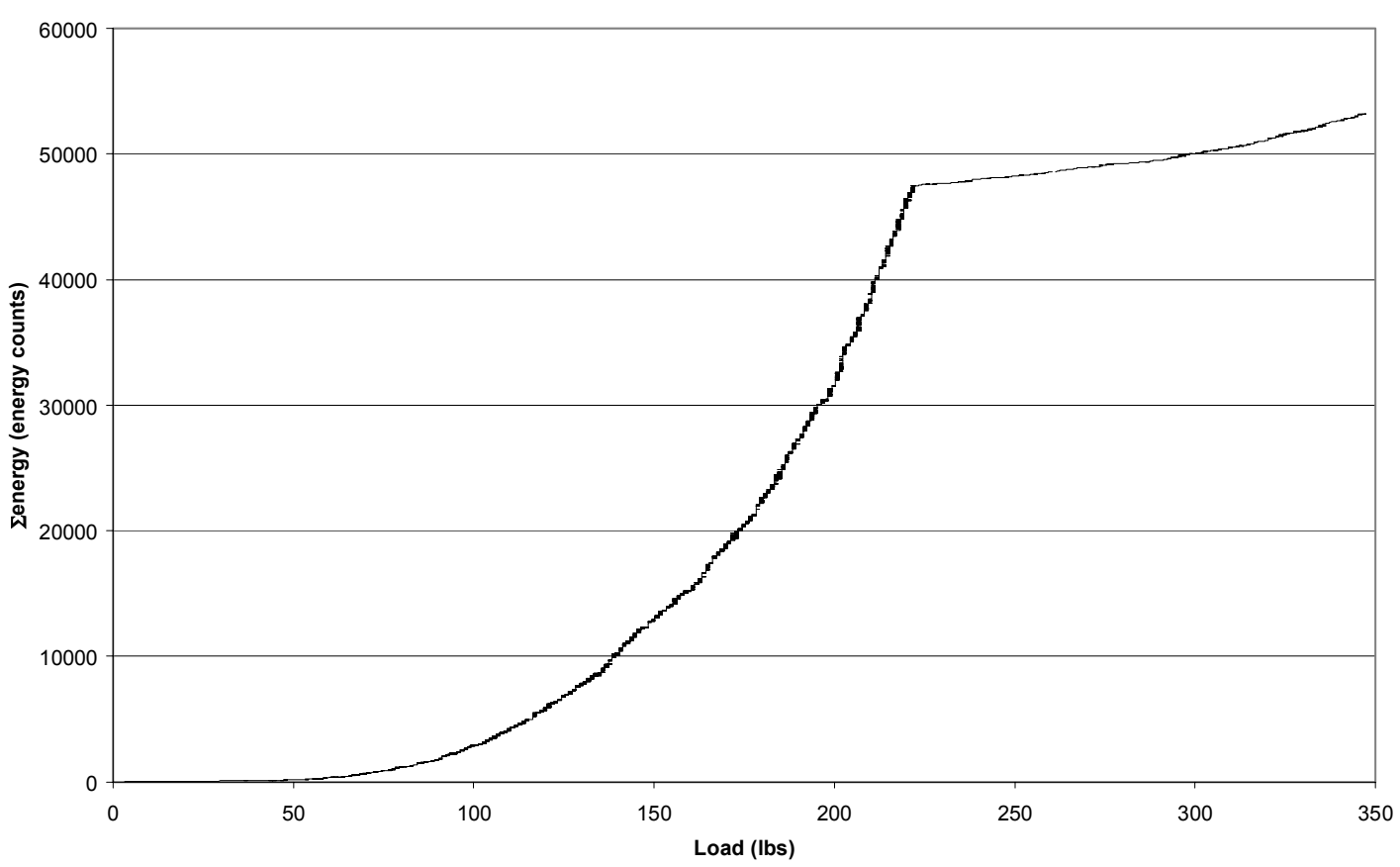

Figure 4.18 $\Sigma$ Energy vs. Load (test B2, failure load $=347.31 \mathrm{lbs}$ )

Test B7, ch 1\&2: Eenergy vs Load

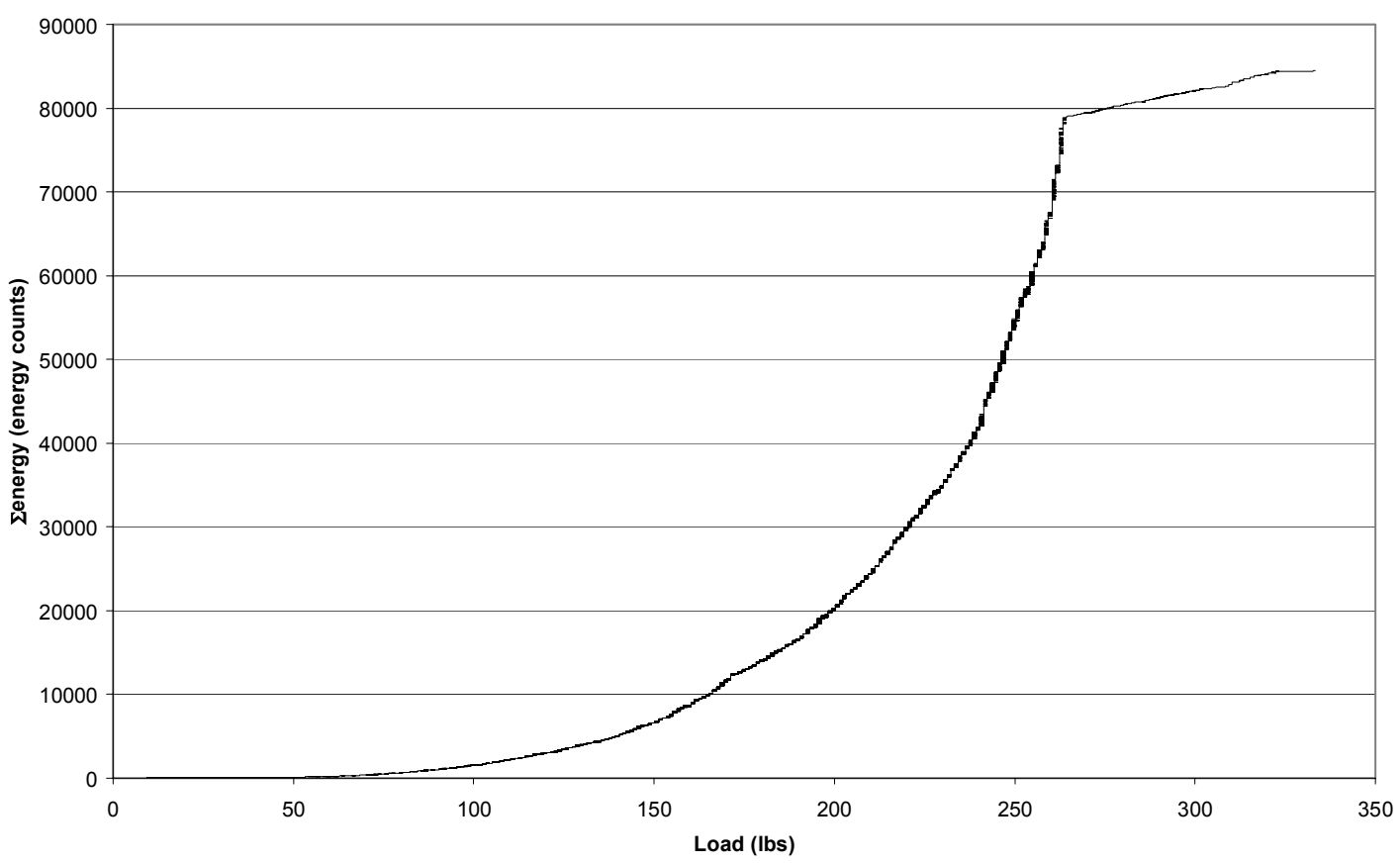

Figure 4.19 $\Sigma$ Energy vs. Load (test B7, failure load = $333.31 \mathrm{lbs}$ ) 


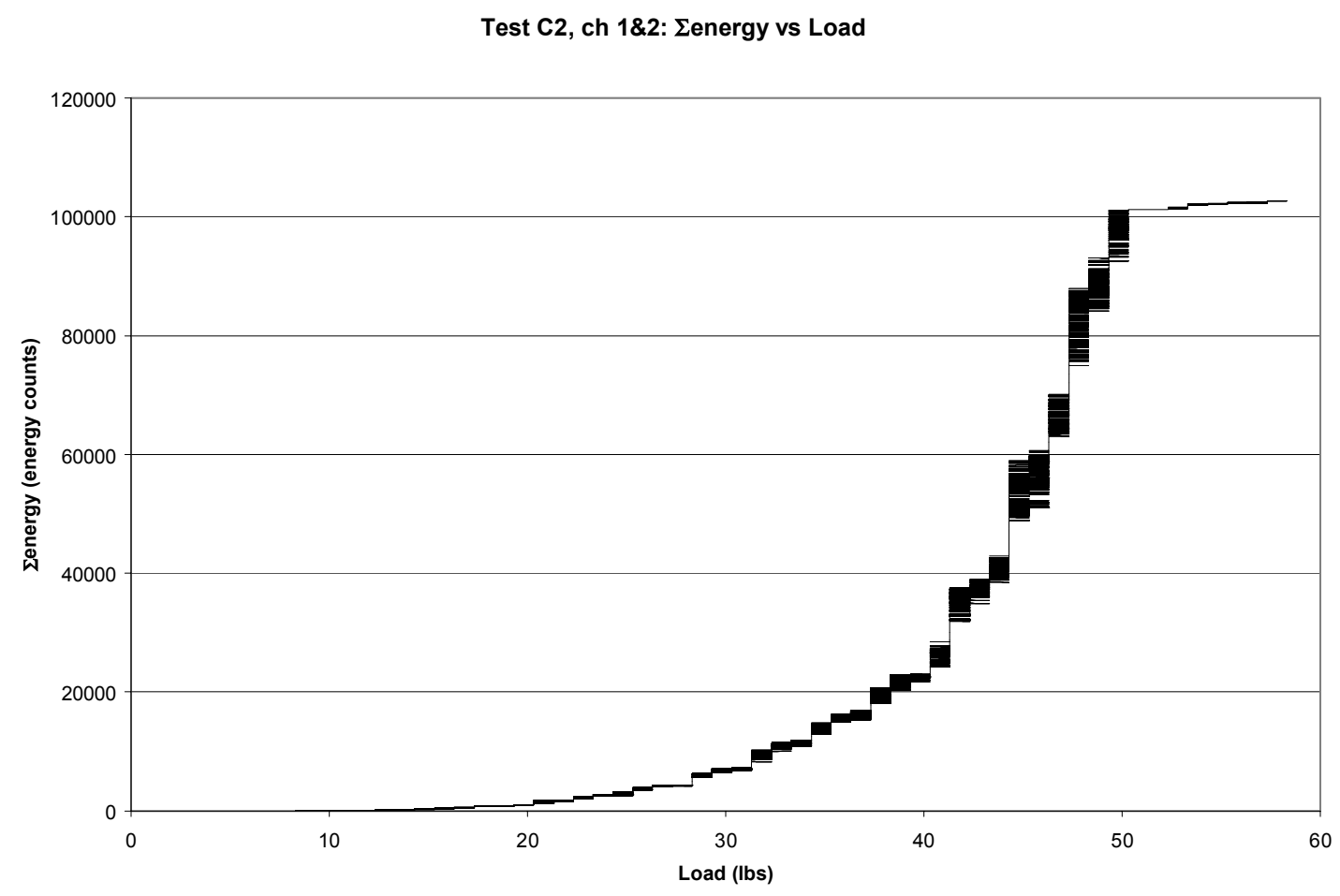

Figure 4.20 $\Sigma$ Energy vs. Load (test C2, failure load $=58.31 \mathrm{lbs}$ ) 


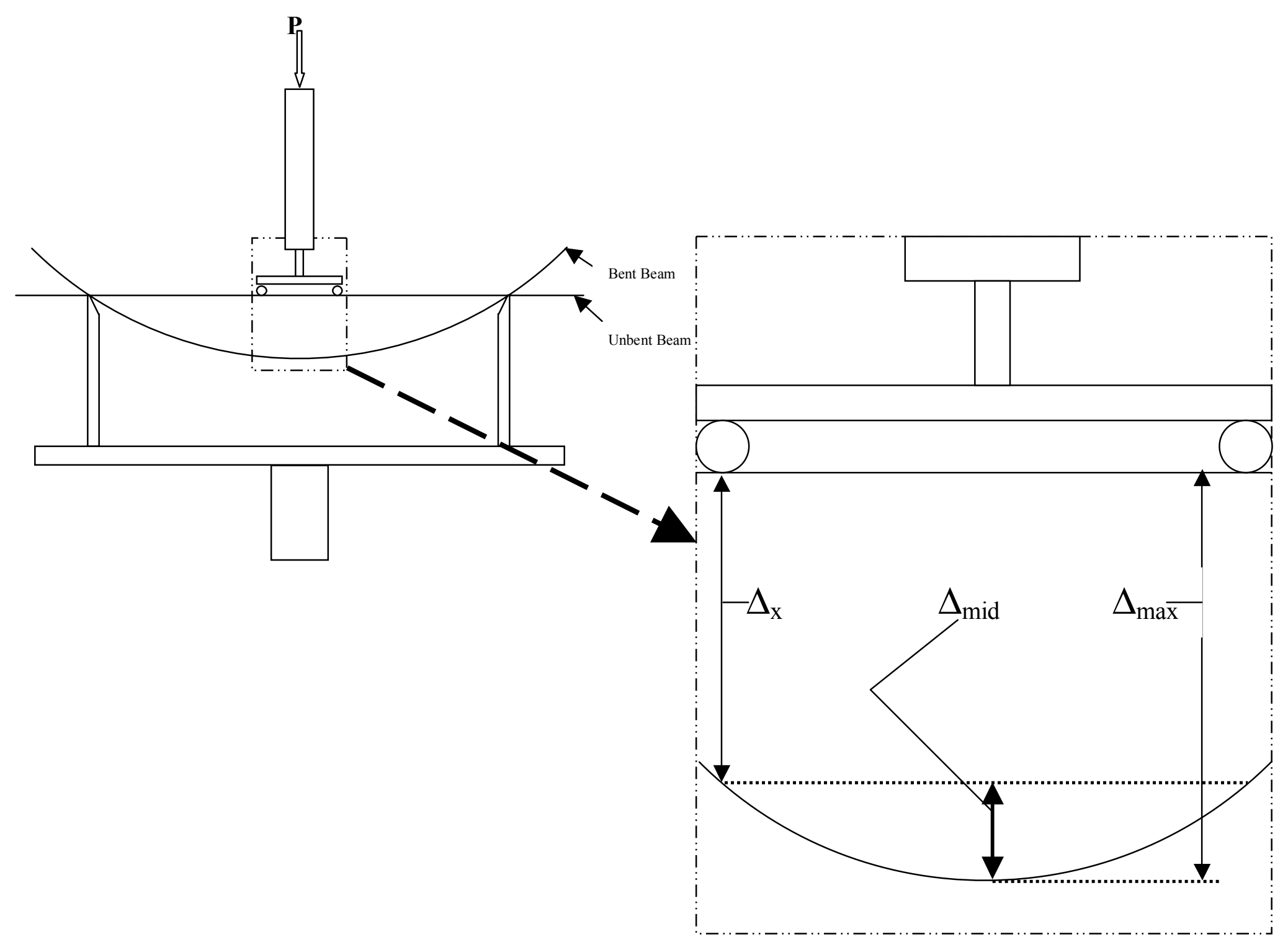

Figure 4.21 Loading Setup and Deflection Measurement Schematic 
Test B1: Load vs Deflection

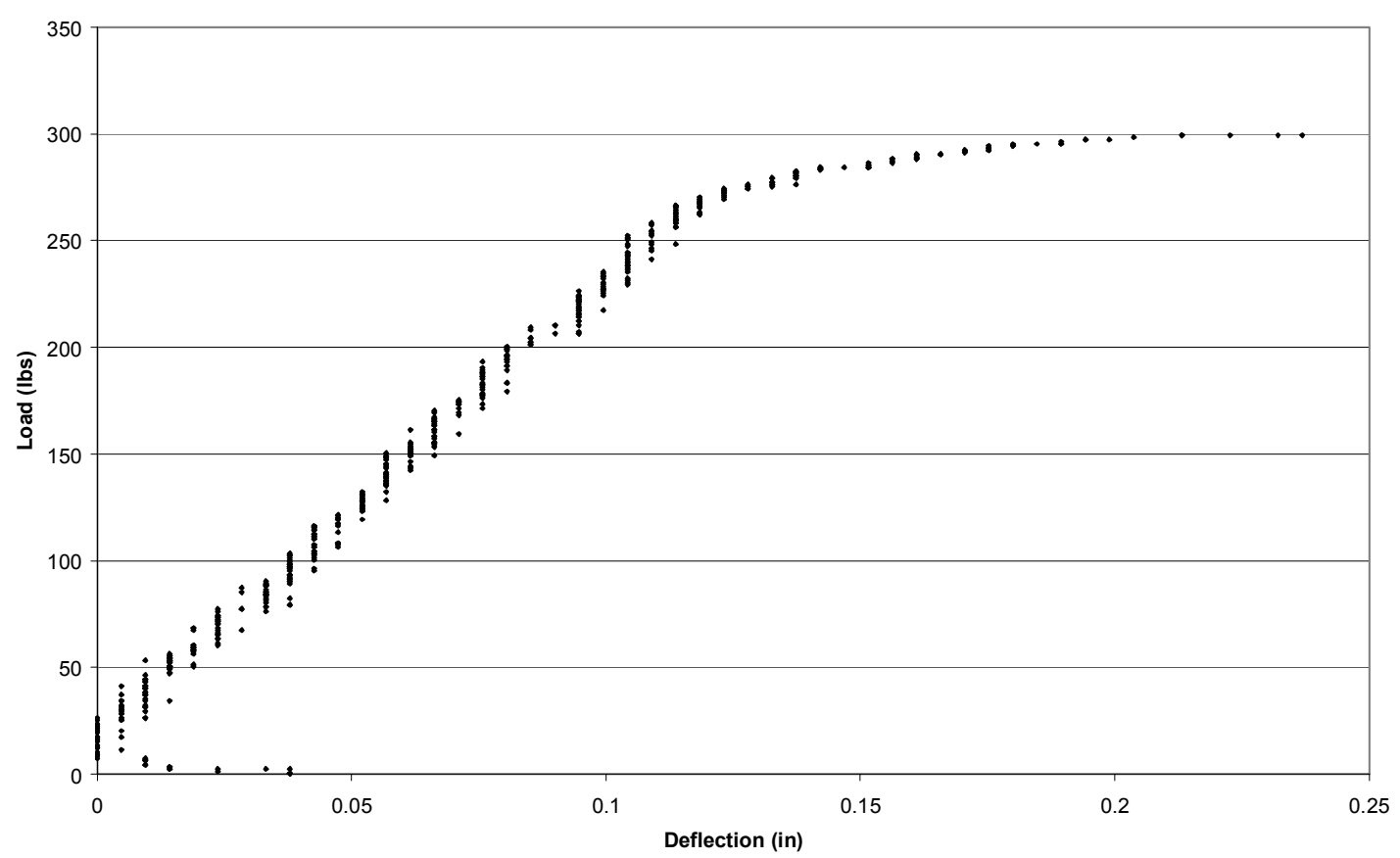

Figure 4.22 Load vs. Deflection Curve (test B1)

Test B7: Load vs Deflection

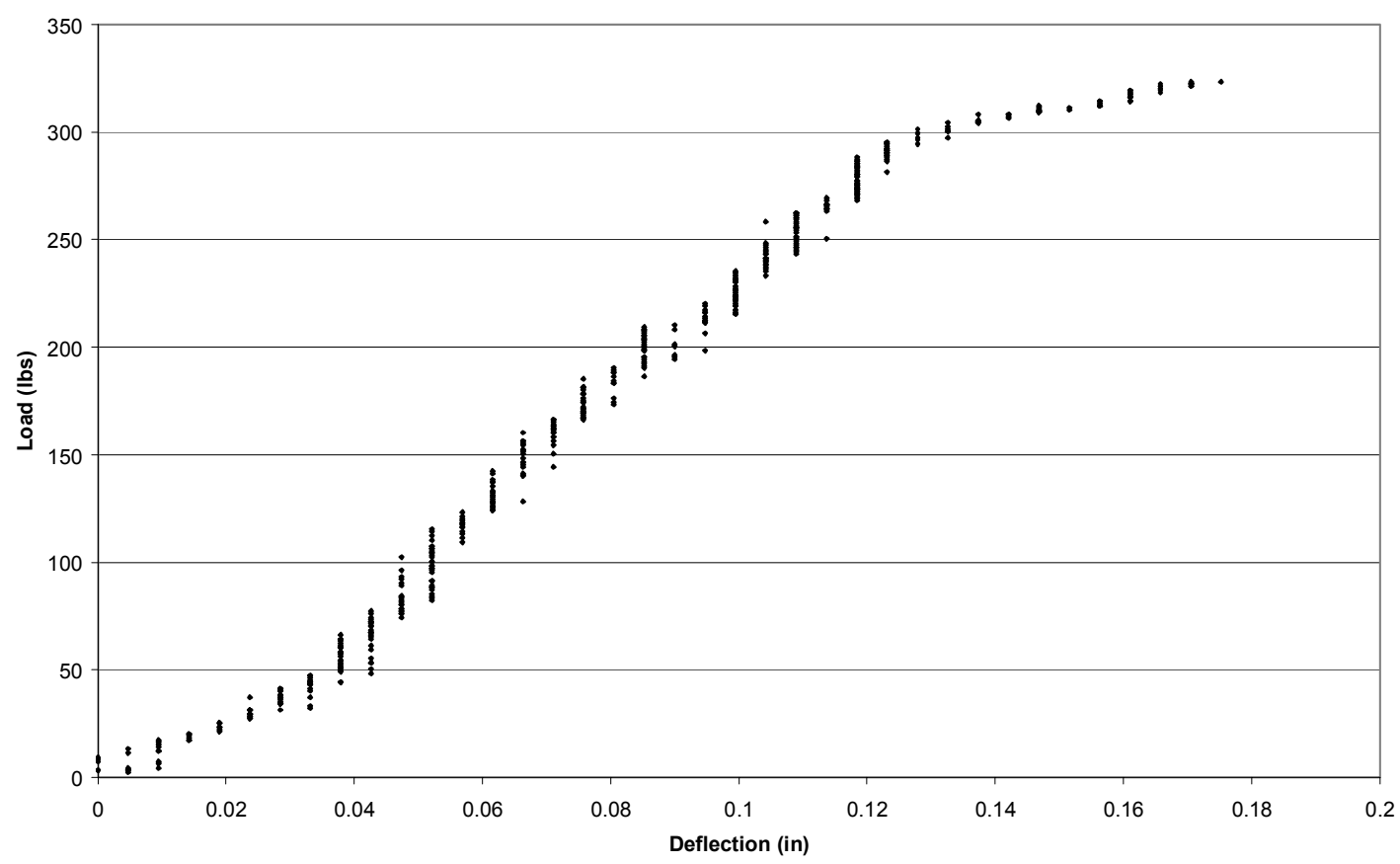

Figure 4.23 Load v. Deflection Curve (test B7) 
Test C4: Load vs Deflection

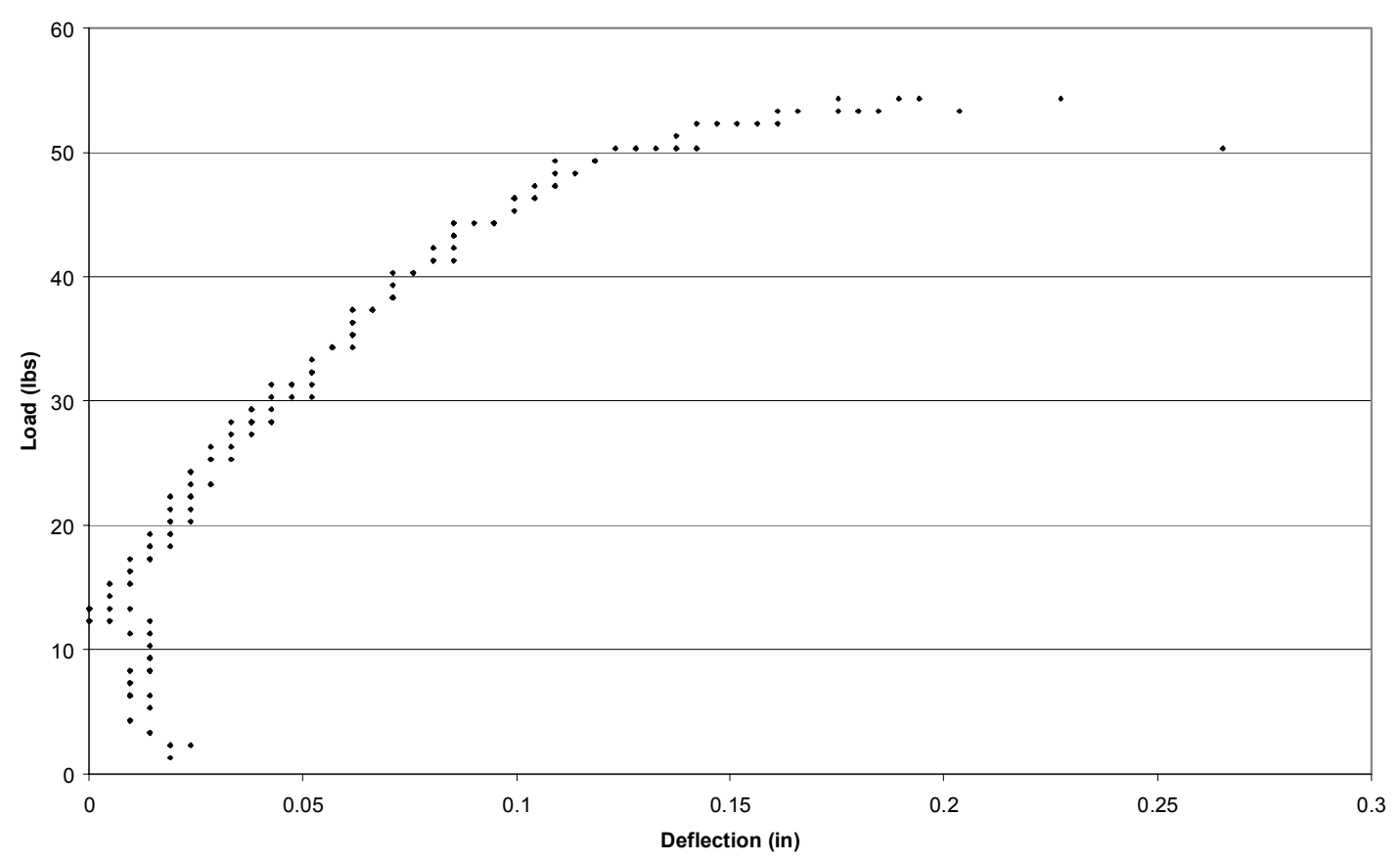

Figure 4.24 Load vs. Deflection Curve (test C4) 


\section{Chapter 5}

\section{Neural Network Analysis of Tension Specimens}

\subsection{Equipment}

\subsubsection{Program}

The program used for this analysis was a Neural Network based program (C.L. Chen 1992). This $\mathrm{C}$ based program runs in MS-DOS. This program uses complicated calculations and many iterations. With modern day computing capabilities, calculations can be done quickly, allowing for the most accurate predictions possible. The program used employs a multi-layer NN system that consists of a set of highly interconnected, nonlinear Processing Units (PU's) that operate in parallel. The connection between any two PU's consists of an adjustable, continuous value termed weight. This weight can be positive (excitatory), negative (inhibitory), or zero. The number of hidden numbers in the hidden layer can be arbitrary; therefore the best method for determining this value is trial and error (Chen \& Chen 1992).

Each PU in the hidden or output layer computes the total input from the summation of the inputs and weights by the following equation.

$$
n e t_{j}=\sum_{i} w_{i j} y_{i}+\theta_{j}
$$

where,

$$
\begin{aligned}
& \text { net }_{\mathrm{j}}=\text { the total input to } P U_{j} \\
& \mathrm{w}_{\mathrm{ij}}=\text { the weight of the connection between } \mathrm{PU}_{\mathrm{i}} \text { and } \mathrm{PU}_{\mathrm{j}} \\
& \mathrm{y}_{\mathrm{i}}=\text { the actual output of } \mathrm{PU}_{\mathrm{i}} \\
& \theta_{\mathrm{j}}=\text { the bias of } \mathrm{PU}_{\mathrm{j}}
\end{aligned}
$$


The value net $t_{j}$ is then transformed through a nonlinear, differentiable sigmoid function to arrive at the actual output. This function is as follows.

$$
y_{j}=f\left(\text { net }_{j}, U_{j}, L_{j}, T_{j}\right)=\frac{U_{j}-L_{j}}{1+e^{-n e t_{j} / T_{j}}}+L_{j}
$$

Where,

$$
\begin{aligned}
& U_{j} \text { and } L_{j}=\text { the upper and lower bounds of the output function, respectively } \\
& T_{j}=\text { the slope of the function }
\end{aligned}
$$

\subsubsection{Computer}

The computer used in this analysis was a Compaq Presario 4090US with a $900 \mathrm{MHz}$ AMD Duron processor and $128 \mathrm{MB}$ of RAM. As can be seen, any personal computer has sufficient capabilities to run this program. This eliminates the need for high priced complicated computer equipment.

\subsubsection{Input Matrices}

The input files, training set or test set, are composed of the following. At the beginning of the file there are three numbers. The first of these is the total number of spots in the body of each matrix. Since the matrices were of a 10 by 10 size, this number was 100 . The second number is the number of slots at the end of each matrix for results. In this case it was just one, loading quarter. The final number is the total number of matrices in the file. For the training set

this number is 24 ( 6 specimens $* 4$ matrices/specimen $=24$ matrices $)$ and 4 for the test set. An example from a training set input file can be seen in Figure 5.1. 


\subsection{Loading Quarter Prediction}

In order to have an effective early warning system for a structure, the loading needs to be predicted. Loading quarter, as in Chapter 3, was also used for this Neural Network analysis. A prediction of the loading quarter of the specimens was analyzed. A prediction of 1 means $1^{\text {st }}$ quarter, 2 means $2^{\text {nd }}$ quarter and so on. Because of this, each specimen's data was split into four sections $; 1^{\text {st }}, 2^{\text {nd }}, 3^{\text {rd }}$, and $4^{\text {th }}$ quarters.

\subsubsection{Training Set Matrices}

A training set does exactly what the name infers it does, train or "teach" the computer. The training set consists of the input matrix joined with the result matrix. These sets are then input into the Neural Network program from common text files. The computer then uses these input matrices to find connections to the output using a hidden layer of analysis. This hidden layer analysis is then later used to predict unknown results for other data sets.

Each training set consisted of 6 of the tests. In order to create these training sets first, parameters for use needed to be chosen. The parameters were chosen based on which ones had the strongest, most distinctive patterns. These parameters were: counts, energy, duration, amplitude, and frequency. In order to organize these visual trends or patterns into numerical matrix form, a method of summation (many events needed to be combined into one) had to be found. Due to a large number of signals of low amplitude throughout the loading, important trends near the end of the loading were being damped out. To solve this problem, the test data for each specimen was filtered. All signals having amplitudes of $60 \mathrm{~dB}$ or less were eliminated. 
Next, each specimen's loading was divided into 40 pieces, or $2.5 \%$ of the failure loading. At each $1 / 40$ point, each parameter needed to provide a single value. To accomplish this, an average for each loading section was calculated. This was done for channels one and two separately for each test. The resulting plots for all of the tests are shown in Figures 5.2-5.5. The seven graphs (one for each specimen) for each specimen are plotted together to show the strong, similar trend exhibited by all of them. The input for frequency was done differently from the other 4 parameters. The frequency input is simply a count of signals that fall into a certain watch area during each increment. Any signal with a frequency between $100 \mathrm{kHz}$ and $400 \mathrm{kHz}$ that had an amplitude of $70 \mathrm{~dB}$ or above was counted. This region, as marked in Figure 5.6, was found to be an indicator region as seen in Chapter 3. A plot of this parameter for each specimen can be seen in Figure 5.7. Note that the graph for test $4 \mathrm{t}$ has a large spike (to about 19) at around 25\% of the loading. One possible cause for this may be an internal specimen flaw.

Normalization makes the data from each test equally influential. For each specimen, a maximum and minimum value for each parameter was established. Each specimen was normalized only to its own data. This was done in order to show the pattern of each specimen the strongest. If one set of maximum and minimum values were set for all specimens, the trends for some of specimens that produced lower average values would be somewhat diluted or drowned out. For example, the maximum average amplitude value found for test $1 \mathrm{t}$ was 79.10 $\mathrm{dB}$ as opposed to a value of $70.84 \mathrm{~dB}$ for test $2 \mathrm{t}$. If the maximum for all tests was set at 79.10 $\mathrm{dB}$, the maximum normalized value for test $2 \mathrm{t}$ would be considerably lower than 1 . The maximum value chosen was then set equal to one and the minimum to zero. All values in between were then converted to numbers between zero and one based on these maximum and minimum values. The out-liers (numbers greater than or less than the maximum and minimum 
values) were set equal to one and zero, respectively. Table 5.1 shows a summary of these maximum and minimum values.

\subsubsection{Test Set Matrices}

The test set is used to test the training of the Neural Networks program. After the computer is trained, the test set is then input to see if the system can predict the results. The program then uses the hidden weights that were found during the training phase to make these predictions. In order to distinguish if the system's prediction is correct, the results of the test set must also be known. The program then calculates the correct rate based on a 40-20-40 rule. If the predicted correct rate falls in the $40 \%$ on either side of the true value it is correct, in the furthest $40 \%$ it is incorrect, and in the middle $20 \%$ it is unknown. For example, if the program is trying to predict $2,1.60$ to 2.40 is considered correct, $1.40-1.60 \& 2.40-2.60$ are unknown. Anything below 1.40 and above 2.60 is incorrect.

A total of 7 trials were conducted. The test set for each of these trials was the data for the specimen that was not used in the training set. For trial 1 , tests $2 \mathrm{t}$ through $7 \mathrm{t}$ were included in the training set and test $1 \mathrm{t}$ was the test set. This was repeated for each of the 7 trials.

\subsubsection{Results}

In order to obtain the best results, different amounts of numbers were tried for the hidden layer. The best value found for the load quarter predictor was 13. Figure 5.8 shows a schematic of the specific architecture of the NN for the load quarter predictor. Each set was trained until a correct rate of $100 \%$ along with a RMS value of .05 was achieved. Table 5.2 shows a summary of the results for the load quarter predictor. The results of each trial along with a summary of the 
total correct rates for each quarter are given. The total correct rate for all quarters is $64.29 \%$. As can be seen, the correct rate for the $1^{\text {st }}$ quarter is not very high $(28.57 \%)$. This was to be expected due to inconsistencies at the beginning of the loading and the lower number of signals received during that time. As the loading quarter increases, the correct rate gets higher until it hits $100 \%$ for the $4^{\text {th }}$ quarter. The load quarter predictor becomes more accurate as the structure nears a more critical point.

\subsection{Damage Zone Prediction}

Due to the difficulties related to locating the final failure in FRP composite specimens, the term damage zone is being used. Although a specimen may fail visibly in one spot, it may also be damaged more severely in a broad area (damage zone). Each specimen consists of three damage zones. These are the area around sensor one, the area around sensor two, and the area directly between the two sensors. The prediction for damage zone is a number between 0 and 2 , with zero equaling the area around sensor 1 and 2 equaling the area around sensor 2 . The load quarter predictor and the damage zone predictor were given a trial while being included in the same training and test sets. It was found that this severely decreased the accuracy of each prediction. Thus, each predictor was run by itself.

\subsubsection{Training Set Matrices}

As with the loading predictor, each training set consisted of the data from 6 specimens. Each matrix consisted of three rows. The first two rows contained the normalized average energy parameter from the load prediction matrices. The final row was found using a new parameter. It was found from signals that are locatable $(\Delta$ time $<$ time needed to travel between 
the two sensors). For each of these signals, it was then determined whether sensor one or sensor two was hit first. This gives an indication of which side the signal emanates from. A curve for each sensor of the sum of the number of times it was hit first vs. the loading percentage was then made. Figure 5.9 shows these curves for each of the seven specimens. From these plots, the difference in the slope was found between the curves for the two sensors using the same method and equations as in Chapter 3. This difference in slope (*100 to help magnify the numbers) was used for the final row in the matrices.

\subsubsection{Test Set Matrices}

As with the load quarter predictor, a total of 7 trials were conducted following the same method. Also the same 40-20-40 rule was used for correct rate calculations.

\subsubsection{Results}

As with the load quarter predictor, different unit numbers were tried for the hidden layer. A schematic drawing of the architecture for the damage zone predictor can be seen in figure 5.10. Each different unit number changed the results very little with 13 once again being the best value. Table 5.3 shows the results for the damage zone predictor. As can be seen, a total correct rate of 78.57 was achieved. The damage zone predictor was more accurate in the first three quarters, with the fourth being the worst. Larger material attenuations and slower wavespeeds result in many true signals being wrongly eliminated. This along with the fact that the material fails in a very complex manner, results in source location problems in the final quarter. The accuracy in the first three quarters will allow for the damage zone to be attended to before the damage gets to critical levels. 


\begin{tabular}{|c|c|c|c|c|c|c|c|c|c|c|c|c|c|c|}
\hline \multirow[t]{3}{*}{ Parameter } & \multicolumn{14}{|c|}{ Training Sets } \\
\hline & \multicolumn{2}{|r|}{ 1t } & \multicolumn{2}{|c|}{$2 t$} & \multicolumn{2}{|c|}{$3 t$} & \multicolumn{2}{|c|}{$4 t$} & \multicolumn{2}{|c|}{$5 t$} & \multicolumn{2}{|c|}{$6 t$} & \multicolumn{2}{|c|}{$7 \mathrm{t}$} \\
\hline & $\min$ & $\max$ & $\min$ & $\max$ & $\min$ & $\max$ & $\min$ & $\max$ & $\min$ & $\max$ & $\min$ & $\max$ & $\min$ & $\max$ \\
\hline Counts & 7.00 & 53.92 & 5.50 & 27.21 & 10.50 & 26.43 & 9.50 & 40.00 & 8.00 & 39.82 & 12.00 & 34.89 & 16.33 & 40.00 \\
\hline Energy (energy counts) & 5.43 & 57.84 & 6.95 & 26.28 & 4.50 & 43.14 & 5.00 & 35.00 & 7.00 & 38.73 & 6.00 & 46.22 & 10.27 & 59.69 \\
\hline Duration ( $\mu$ seconds) & 24.43 & 402.11 & 27.75 & 105.72 & 40.00 & 186.86 & 30.00 & 280.00 & 48.64 & 246.00 & 52.00 & 229.61 & 82.00 & 355.00 \\
\hline Amplitude (dB) & 62.50 & 79.10 & 62.50 & 70.84 & 62.00 & 72.00 & 62.00 & 74.25 & 62.00 & 72.00 & 62.00 & 74.76 & 65.00 & 77.62 \\
\hline Frequency (hits) & 2.00 & 40.00 & 2.00 & 94.00 & 1.00 & 17.00 & 1.00 & 9.00 & 1.00 & 10.00 & 2.00 & 40.00 & 2.00 & 33.00 \\
\hline
\end{tabular}

Table 5.1 Maximum/Minimum Values for Normalization 


\begin{tabular}{|c|c|c|}
\hline t. & 10 & \\
\hline \hline known & predicted & difference \\
\hline 1 & 1.51 & 0.51 \\
\hline 2 & 1.4 & -0.60 \\
\hline 3 & 2.76 & -0.24 \\
\hline 4 & 3.66 & -0.34 \\
\hline CR & 50 & \multicolumn{1}{|c}{} \\
\cline { 1 - 2 } & \multicolumn{2}{|c}{}
\end{tabular}

\begin{tabular}{|c|c|c|}
\hline \multicolumn{3}{|c|}{24} \\
\hline known & predicted & difference \\
\hline 1 & 0.58 & -0.42 \\
\hline 2 & 2.36 & 0.36 \\
\hline 3 & 3.38 & 0.38 \\
\hline 4 & 3.7 & -0.30 \\
\hline CR & 75 & \\
\hline
\end{tabular}

\begin{tabular}{|c|c|c|}
\hline \multicolumn{3}{|c|}{31} \\
\hline known & predicted & difference \\
\hline 1 & 1.14 & 0.14 \\
\hline 2 & 1.77 & -0.23 \\
\hline 3 & 2.9 & -0.10 \\
\hline 4 & 3.77 & -0.23 \\
\hline CR & 100 & \\
\hline
\end{tabular}

\begin{tabular}{|c|c|c|}
\hline \multirow{2}{*}{} & $\mathbf{4 t}$, & \\
\hline \hline known & predicted & difference \\
\hline 1 & 1.81 & 0.81 \\
\hline 2 & 2.81 & 0.81 \\
\hline 3 & 3.78 & 0.78 \\
\hline 4 & 3.76 & -0.24 \\
\hline CR & 25 & \multicolumn{1}{|c}{} \\
\cline { 1 - 2 } & \multicolumn{2}{|c}{} \\
\cline { 1 - 2 } &
\end{tabular}

\begin{tabular}{|c|c|c|}
\hline \multicolumn{3}{|c|}{51} \\
\hline known & predicted & difference \\
\hline 1 & 1.41 & 0.41 \\
\hline 2 & 2.23 & 0.23 \\
\hline 3 & 2.93 & -0.07 \\
\hline 4 & 3.69 & -0.31 \\
\hline CR & 75 & \\
\hline
\end{tabular}

\begin{tabular}{|c|c|c|}
\hline \multicolumn{3}{|c|}{61} \\
\hline known & predicted & difference \\
\hline 1 & 1.26 & 0.26 \\
\hline 2 & 2.9 & 0.90 \\
\hline 3 & 3.39 & 0.39 \\
\hline$\overline{4}$ & 3.94 & -0.06 \\
\hline CR & 75 & \\
\hline
\end{tabular}

\begin{tabular}{|c|c|c|}
\hline \multicolumn{3}{|c|}{$7 t$} \\
\hline known & predicted & difference \\
\hline 1 & 0.29 & -0.71 \\
\hline 2 & 2.33 & 0.33 \\
\hline 3 & 3.64 & 0.64 \\
\hline 4 & 3.95 & -0.05 \\
\hline CR & 50 & \\
\hline
\end{tabular}

\begin{tabular}{l}
\hline \multicolumn{2}{|c|}{ Summary } \\
\begin{tabular}{|c|c|}
\hline \hline Quarter & CR \\
\hline \hline 1 & 28.57 \\
\hline 2 & 57.14 \\
\hline 3 & 71.43 \\
\hline 4 & 100 \\
\hline Total & 64.29 \\
\hline
\end{tabular}
\end{tabular}

Table 5.2 Results for NN Load Quarter Predictor 


\begin{tabular}{|c|c|c|}
\hline \multicolumn{1}{|c|}{} & \multicolumn{1}{|c|}{} \\
\hline \hline known & predicted & difference \\
\hline 1 & 1.19 & 0.19 \\
\hline 2 & 1.99 & -0.01 \\
\hline 2 & 1.94 & -0.06 \\
\hline 1 & 0.7 & -0.30 \\
\hline CR & 100 & \multicolumn{1}{|c}{} \\
\cline { 1 - 2 } & &
\end{tabular}

\begin{tabular}{|c|c|c|}
\hline \multicolumn{1}{|c|}{ 4t } \\
\hline \hline known & predicted & difference \\
\hline 1 & 0.7 & -0.30 \\
\hline 1 & 1.22 & 0.22 \\
\hline 1 & 1.96 & 0.96 \\
\hline 1 & 0.18 & -0.82 \\
\hline CR & 50 & \multicolumn{1}{|c}{} \\
\cline { 1 - 2 } & \multicolumn{3}{|c}{} \\
\hline \multicolumn{2}{|c}{}
\end{tabular}

\begin{tabular}{|c|c|c|}
\hline \multicolumn{2}{|c|}{$2 \mathrm{2}$} \\
\hline \hline known & predicted & difference \\
\hline 1 & 0.93 & -0.07 \\
\hline 2 & 0.91 & -1.09 \\
\hline 2 & 1.77 & -0.23 \\
\hline 2 & 2 & 0.00 \\
\hline CR & 75 & \multicolumn{1}{|c}{} \\
\cline { 1 - 2 } & \multicolumn{2}{|c}{}
\end{tabular}

\begin{tabular}{|c|c|c|}
\hline \multicolumn{3}{|c|}{$5 t$} \\
\hline known & predicted & difference \\
\hline 1 & 0.86 & -0.14 \\
\hline 0 & 0.02 & 0.02 \\
\hline 0 & 0.38 & 0.38 \\
\hline 0 & 0.25 & 0.25 \\
\hline CR & 100 & \\
\hline
\end{tabular}

\begin{tabular}{|c|c|c|}
\hline \multicolumn{3}{|c|}{$7 \mathrm{t}$} \\
\hline known & predicted & difference \\
\hline 1 & 1.48 & 0.48 \\
\hline 2 & 1.97 & -0.03 \\
\hline 2 & 1.92 & -0.08 \\
\hline 2 & 0.79 & -1.21 \\
\hline CR & 50 & \\
\hline
\end{tabular}

\begin{tabular}{|c|c|}
\hline \multicolumn{2}{|c|}{ Summary } \\
\hline Quarter & $\overline{C \text { CR }}$ \\
\hline 1 & 85.71 \\
\hline 2 & 85.71 \\
\hline 3 & 85.71 \\
\hline 4 & 57.14 \\
\hline Total & 78.57 \\
\hline
\end{tabular}

\begin{tabular}{|c|c|c|}
\hline \multicolumn{1}{|c|}{ 3t } & \\
\hline \hline known & predicted & difference \\
\hline 1 & 1.19 & 0.19 \\
\hline 0 & 0.19 & 0.19 \\
\hline 0 & 0.13 & 0.13 \\
\hline 0 & 0.04 & 0.04 \\
\hline CR & 100 & \multicolumn{1}{|c}{} \\
\cline { 1 - 2 } & \multicolumn{2}{|c}{}
\end{tabular}

\begin{tabular}{|c|c|c|}
\hline \multicolumn{3}{|c|}{$6 \mathrm{t}$} \\
\hline known & predicted & difference \\
\hline 1 & 0.88 & -0.12 \\
\hline 0 & 0.06 & 0.06 \\
\hline 0 & 0.07 & 0.07 \\
\hline 0 & 0.75 & 0.75 \\
\hline CR & 75 & \\
\hline
\end{tabular}

.



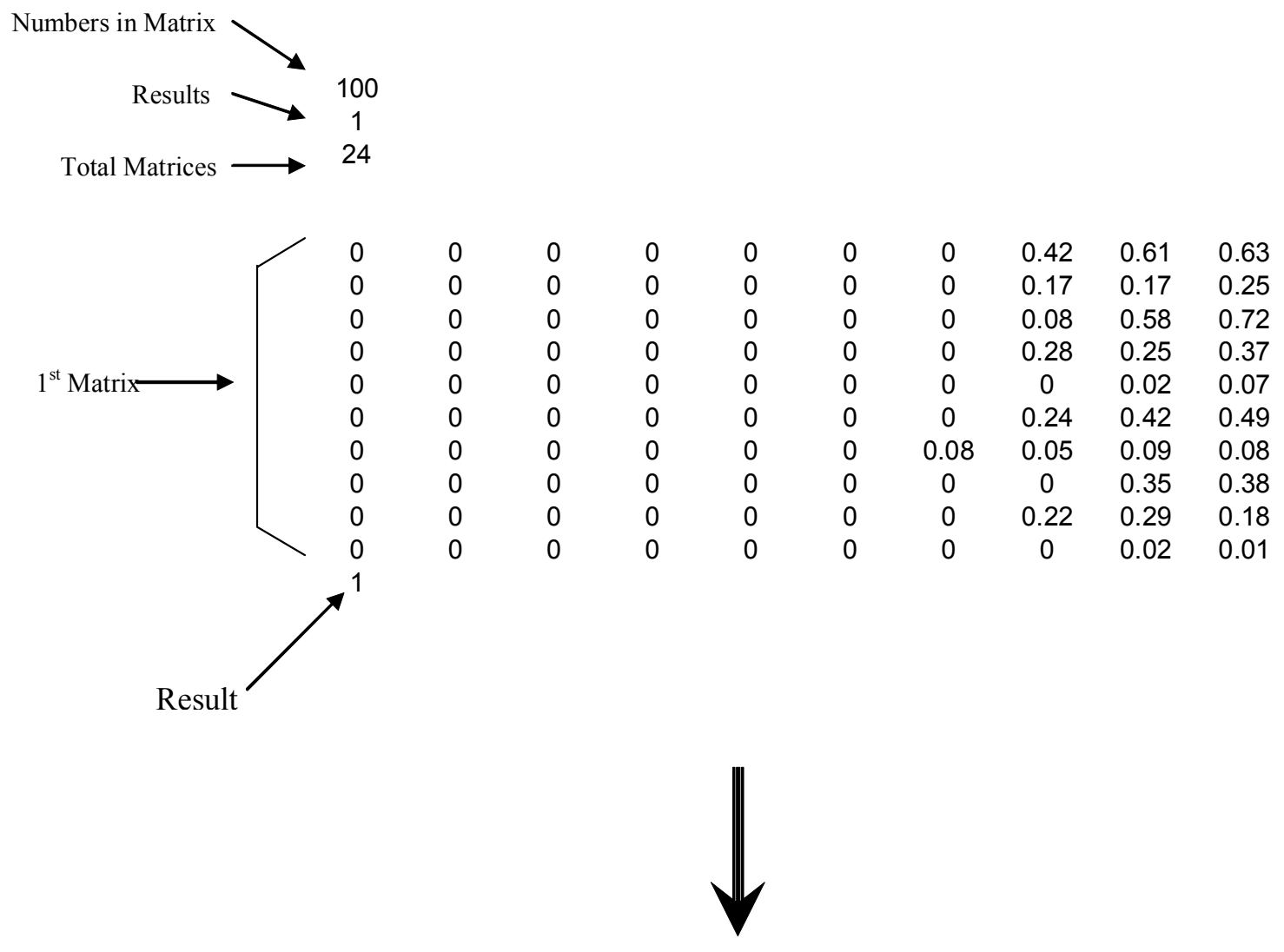

23 Matrix/Result Sets

Figure 5.1 Example from Input File 

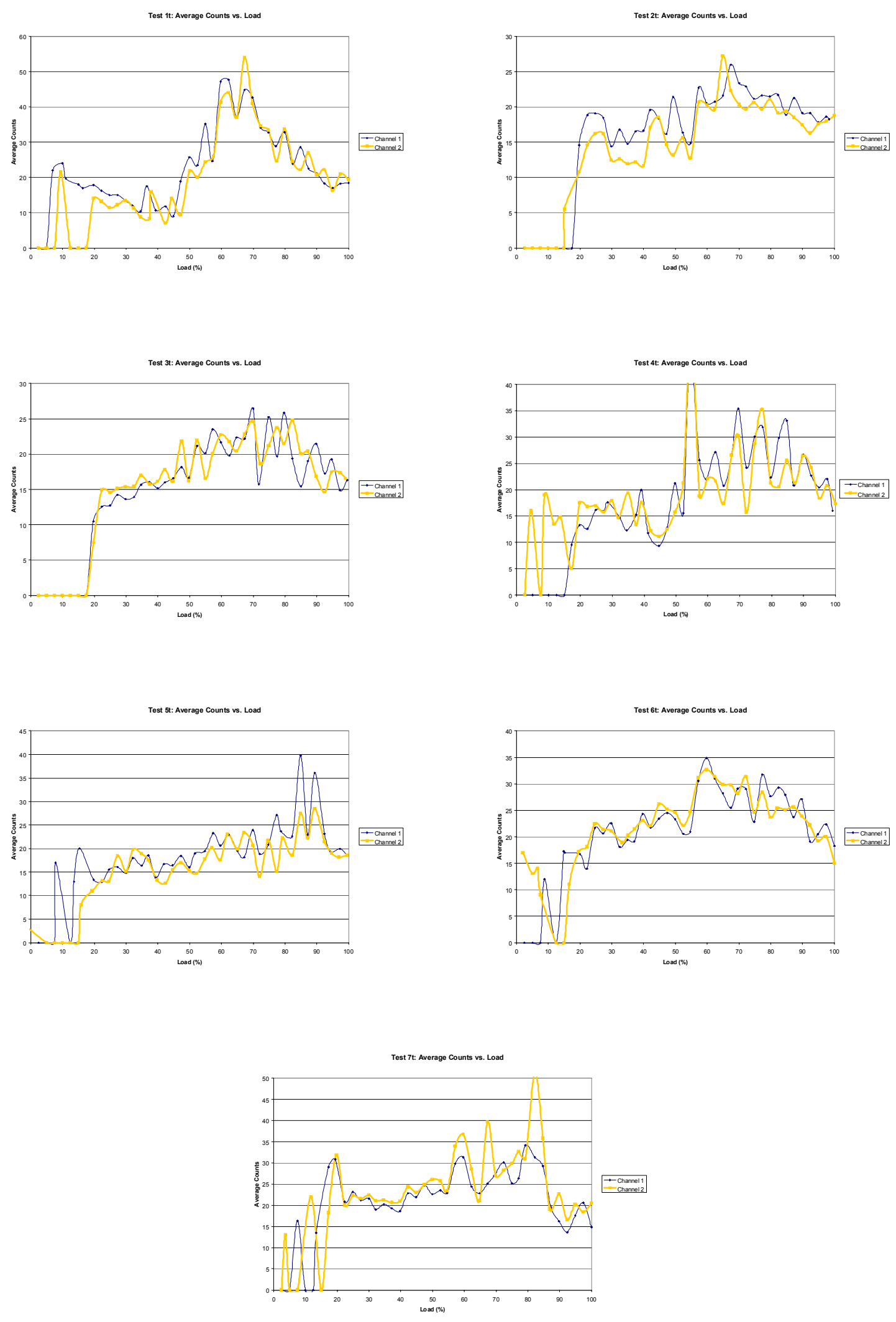

Figure 5.2 Average Counts vs. Load (tests 1t-7t) 

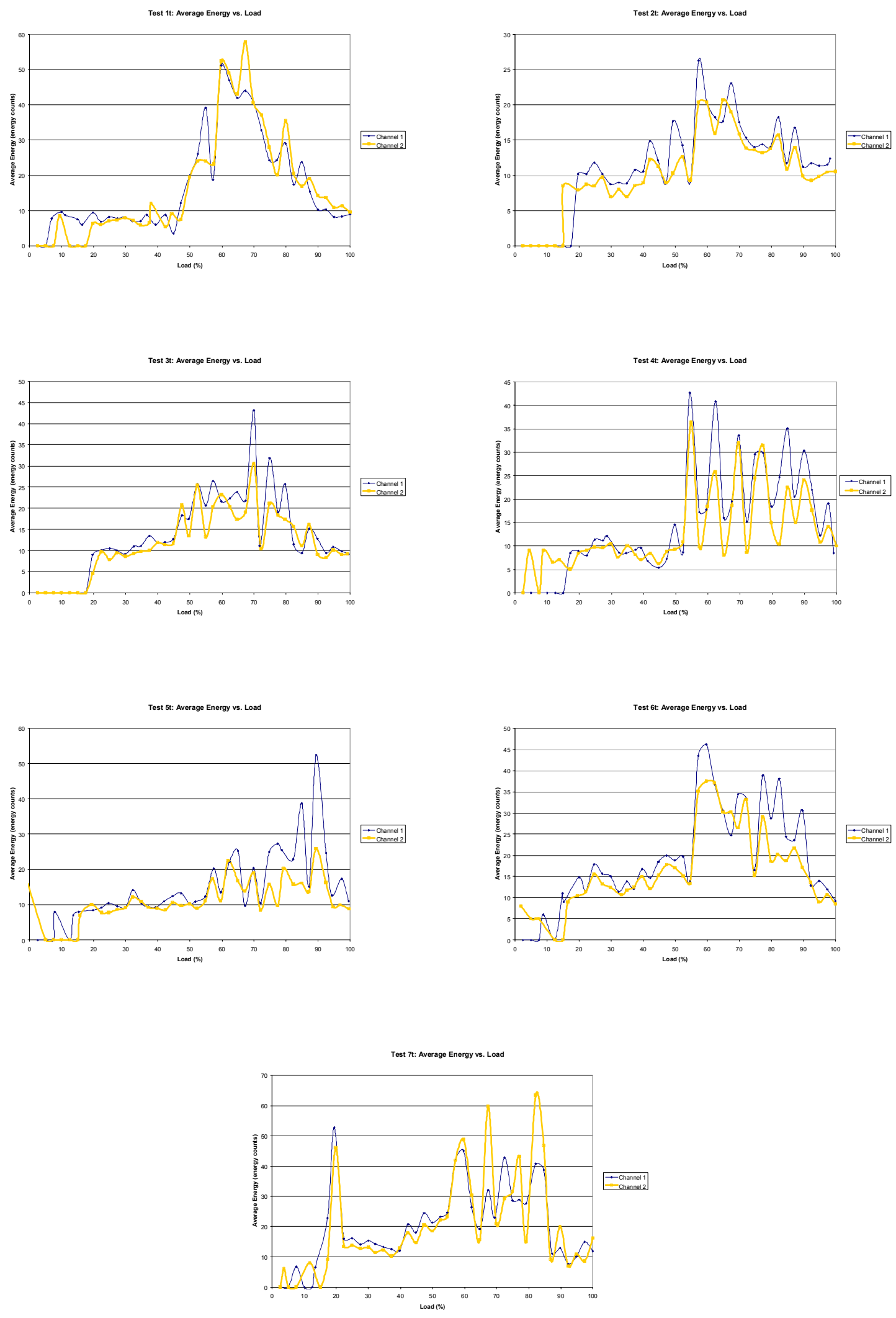

Figure 5.3 Average Energy vs. Load (tests 1t-7t) 
Test 1t: Average Duration vs. Load

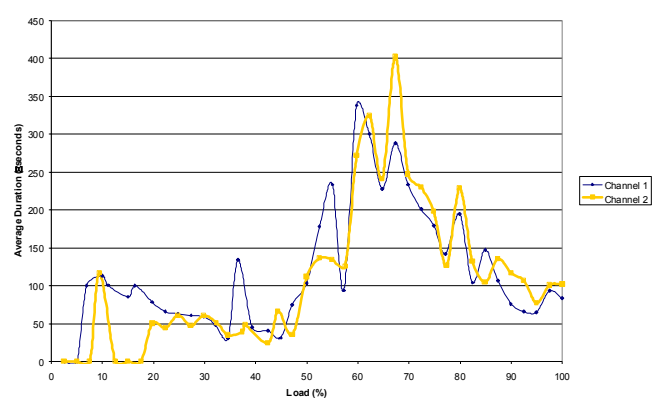

Test 3t: Average Duration vs. Load

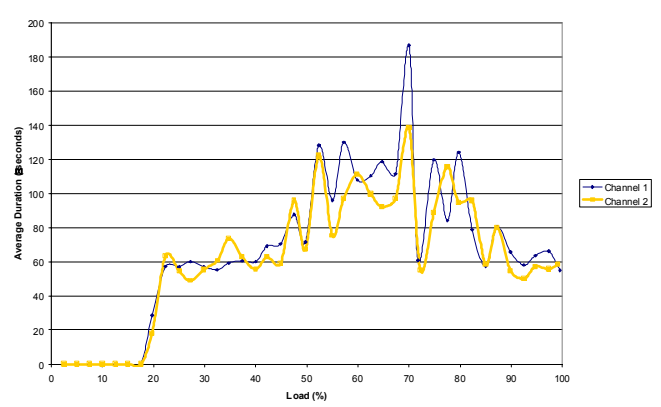

Test 5t: Average Duration vs. Load

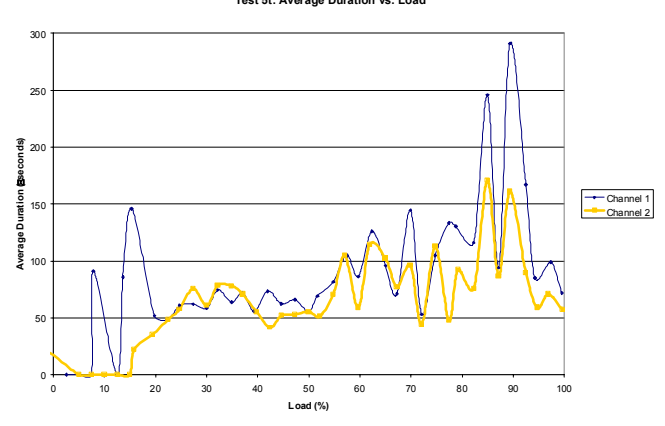

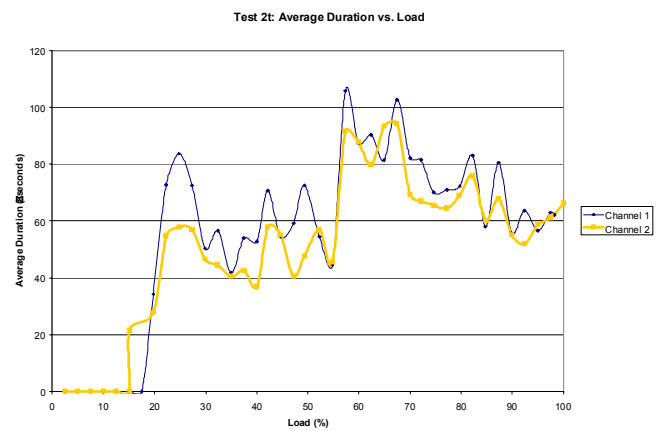
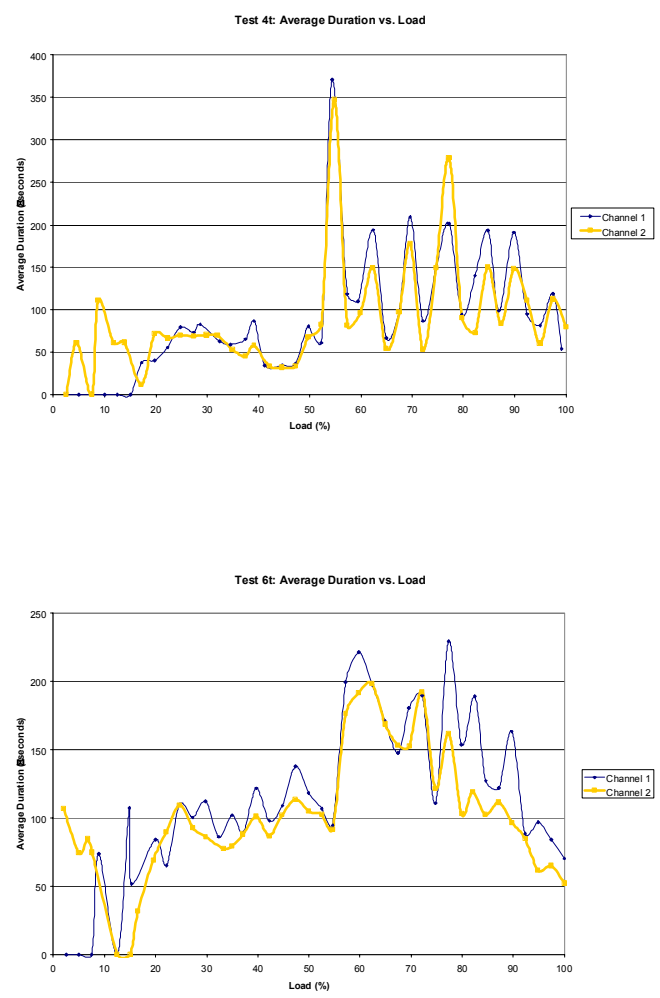

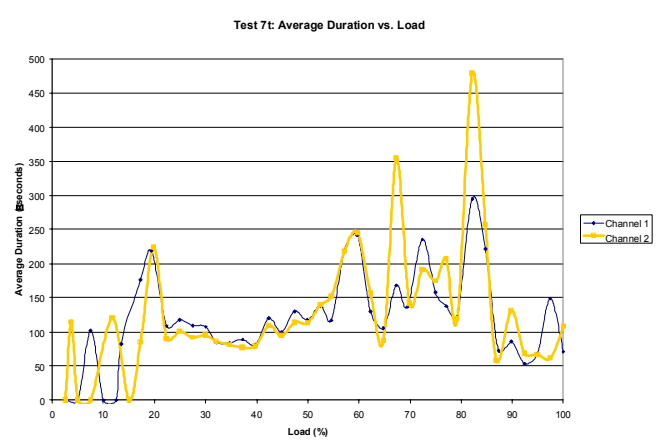

Figure 5.4 Average Duration vs. Load (tests 1t-7t) 

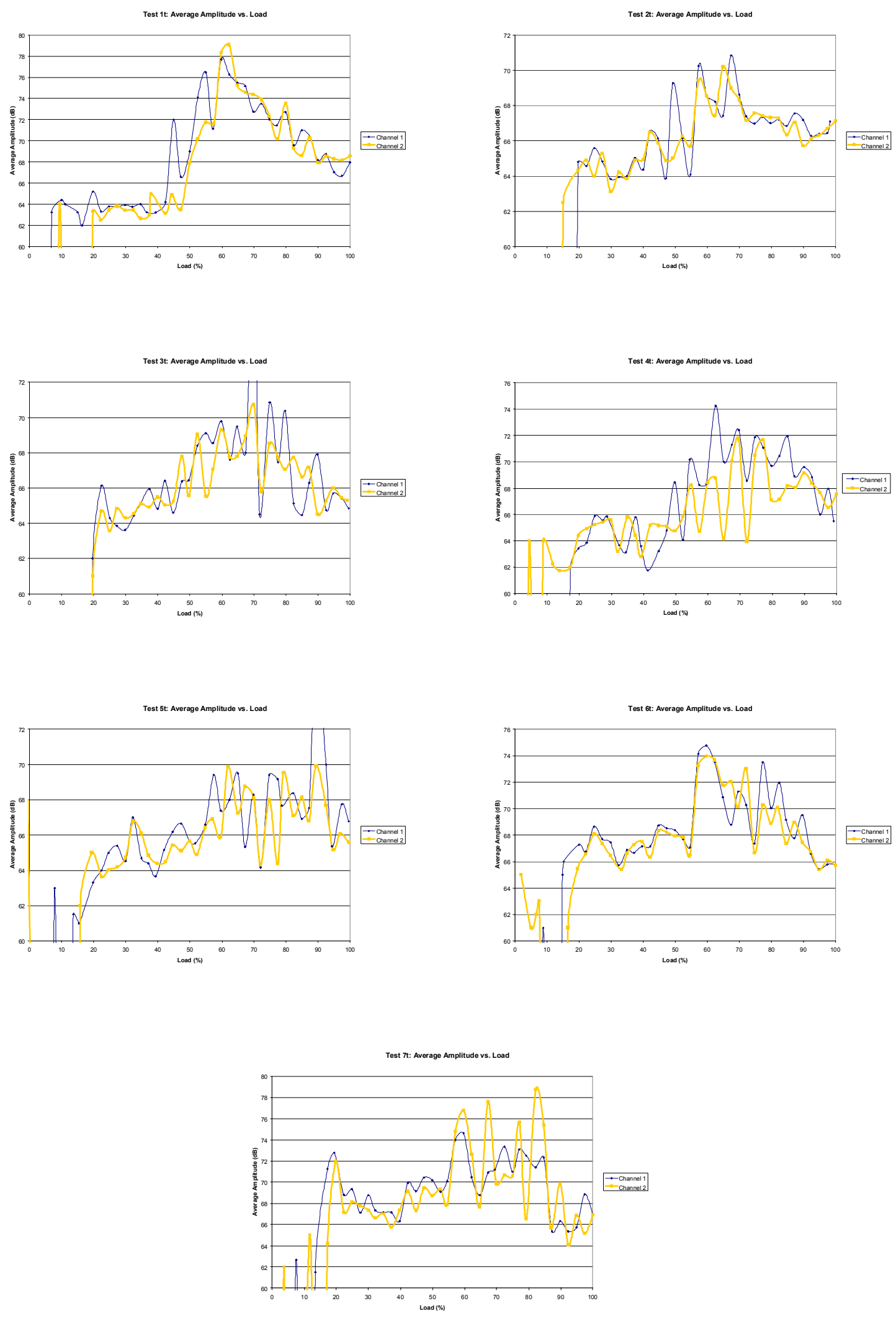

Figure 5.5 Average Amplitude vs. Load (tests 1t-7t) 


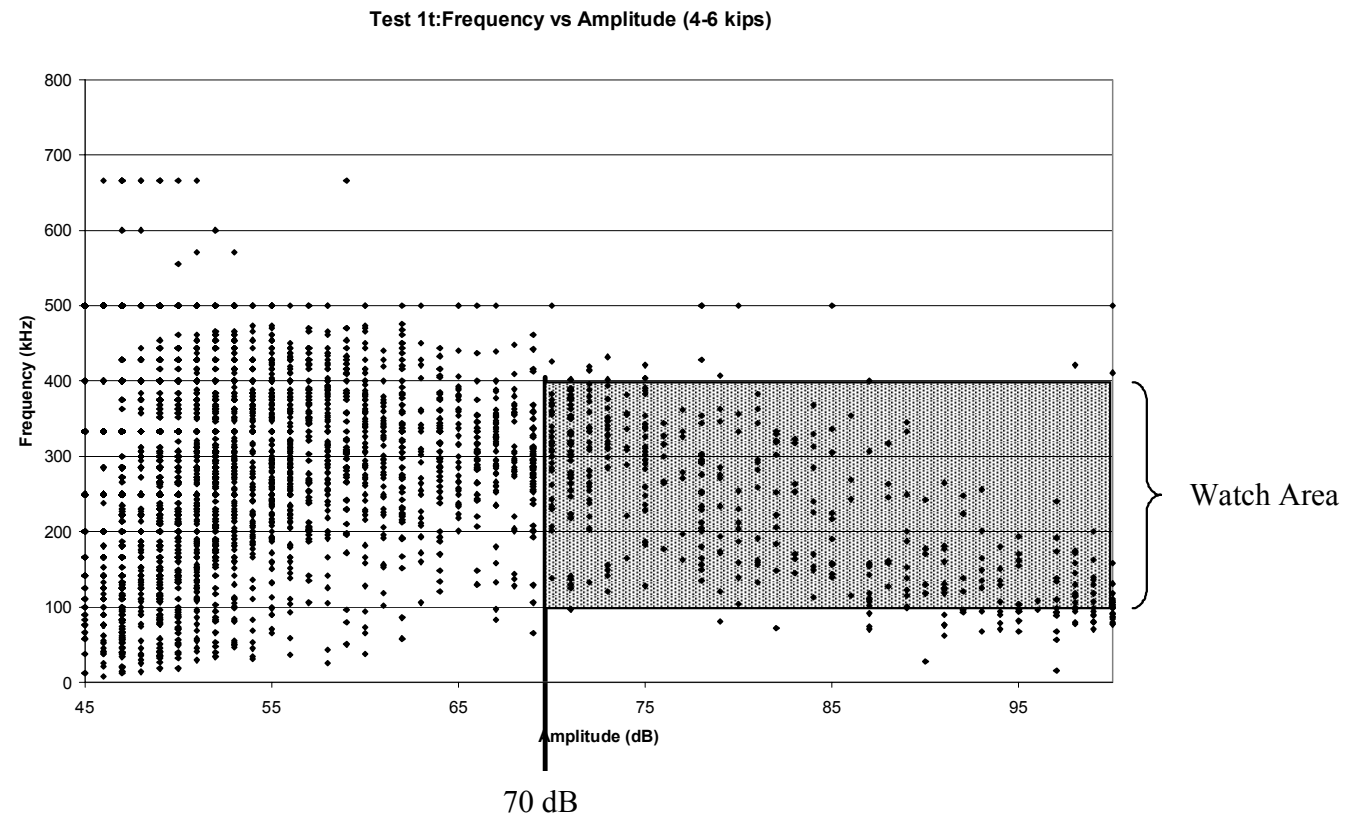

Figure 5.6 Area of Interest on Frequency vs. Amplitude Plots 

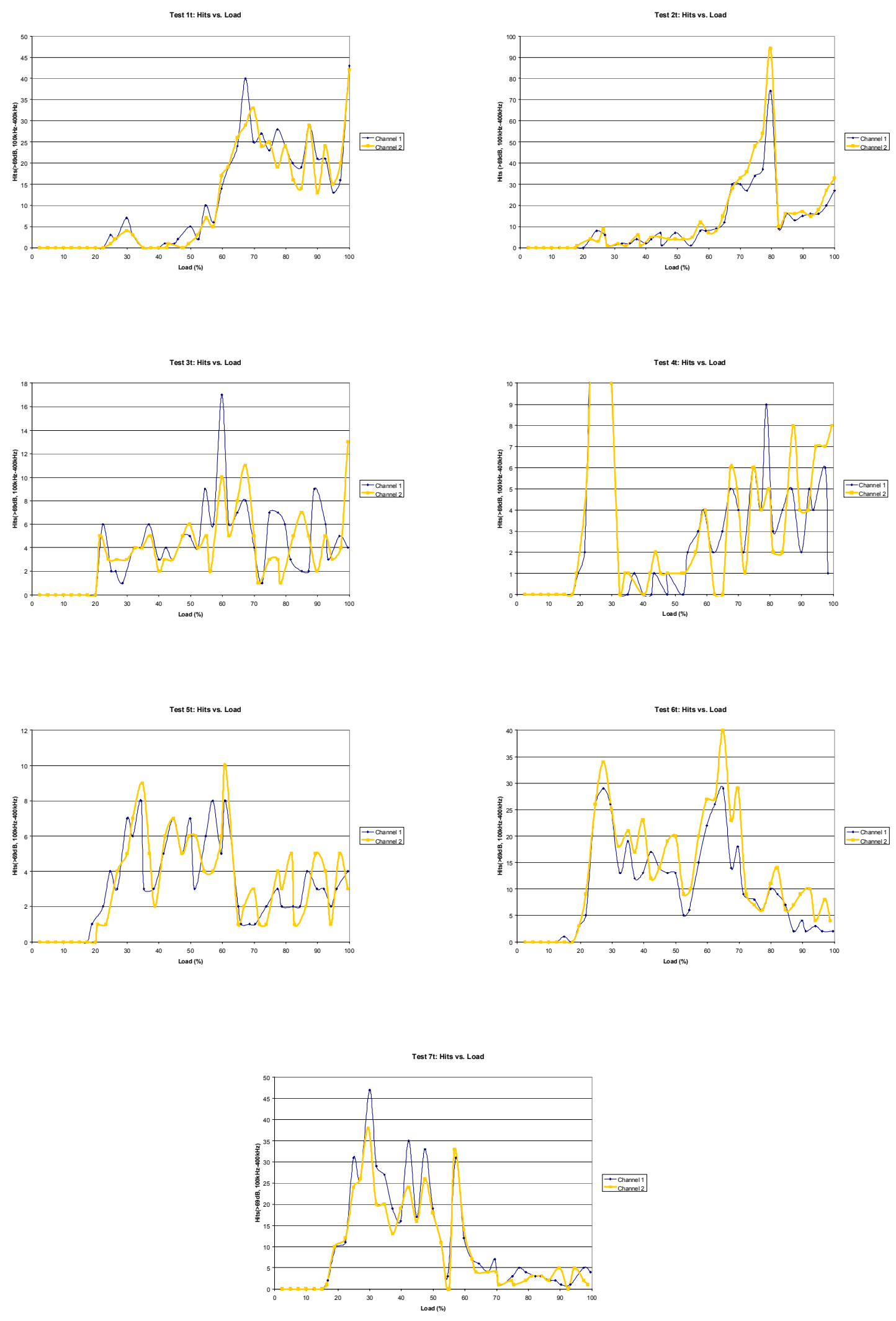

Figure 5.7 Hits ( $>69 \mathrm{~dB}, 100 \mathrm{kHz}-400 \mathrm{kHz})$ vs. Load (tests $1 \mathrm{t}-7 \mathrm{t})$ 


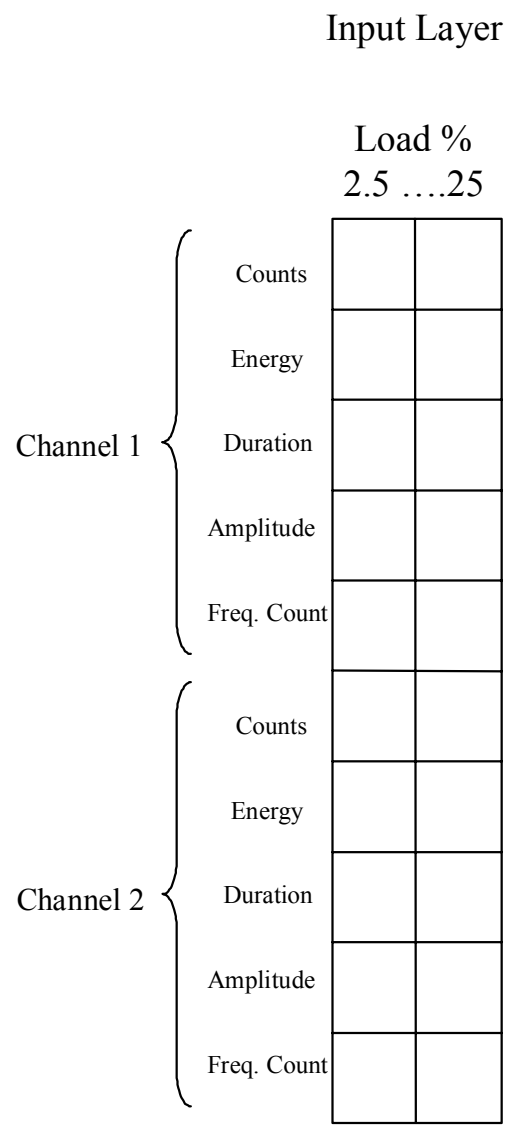

Hidden Layer Output Layer
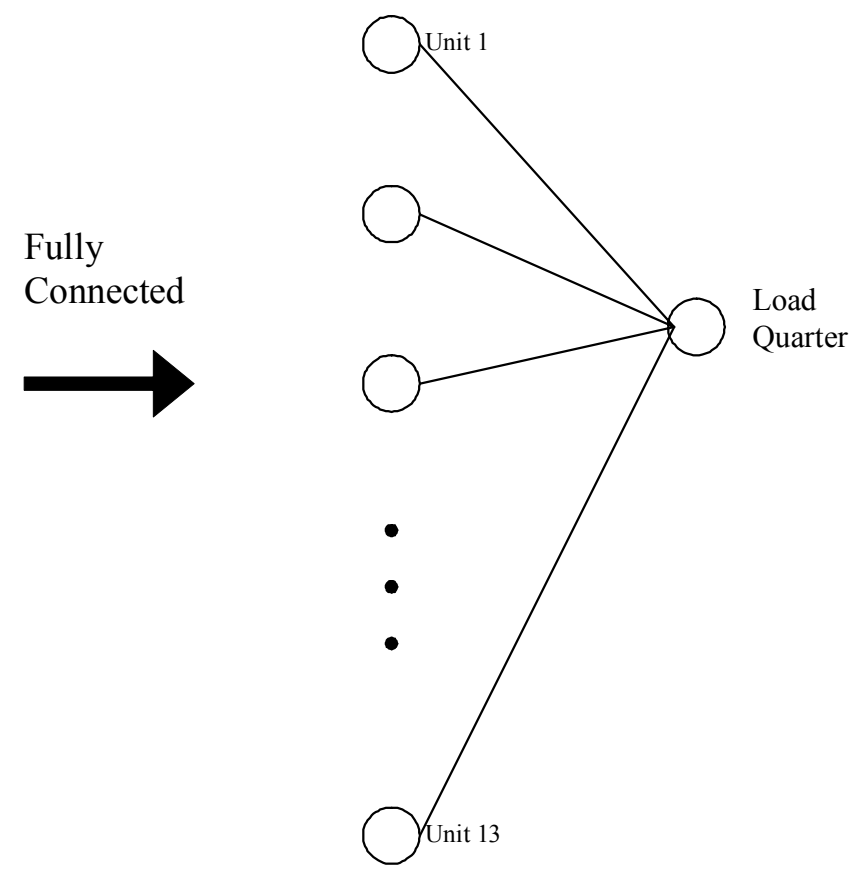

Figure 5.8 NN Architecture for Load Quarter Predictor 
Test 1t: Sum of 1st Sensor Hit

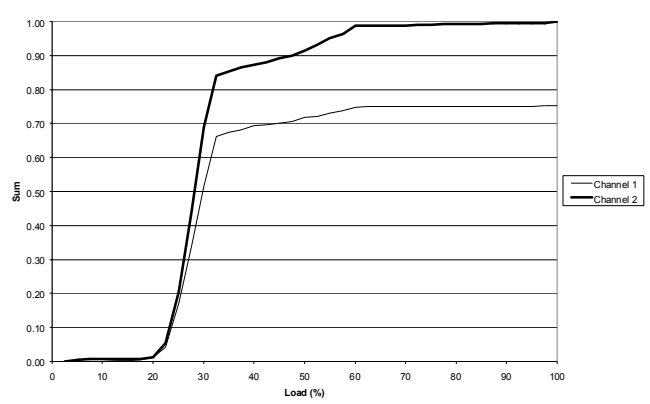

Test 3t: Sum of 1st Sensor Hit

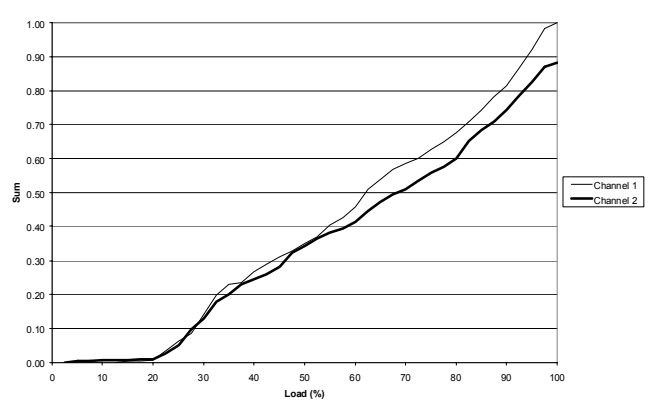

Test 5t: Sum of 1 st Sensor Hit

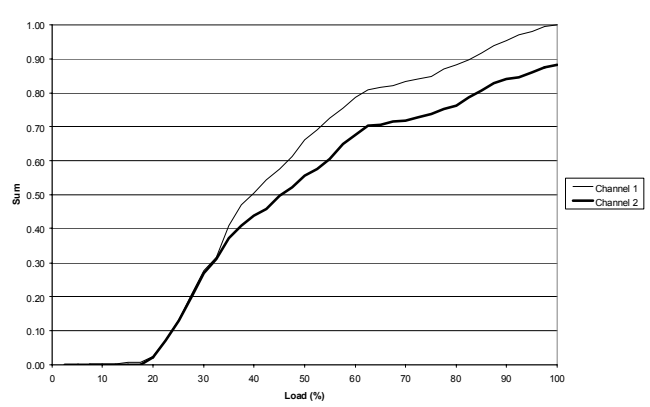

Test 2t: Sum of 1st Sensor Hit

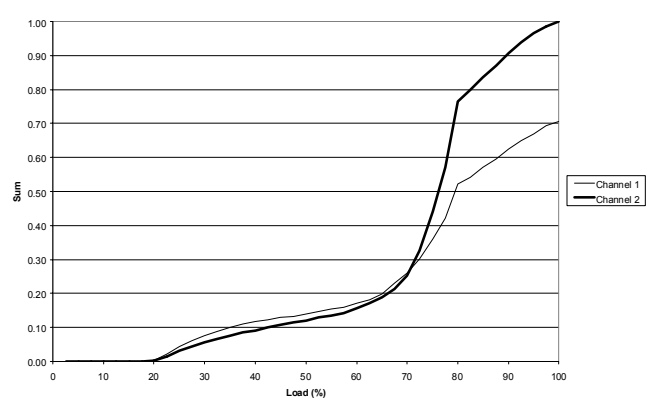

Test 4t: Sum of 1 st Sensor Hit

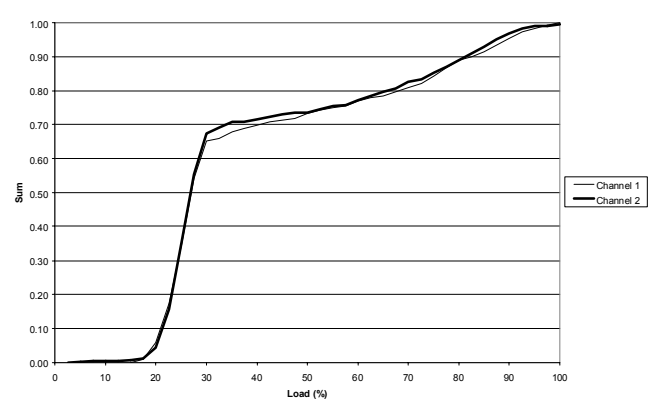

Test 6t: Sum of 1st Sensor Hit

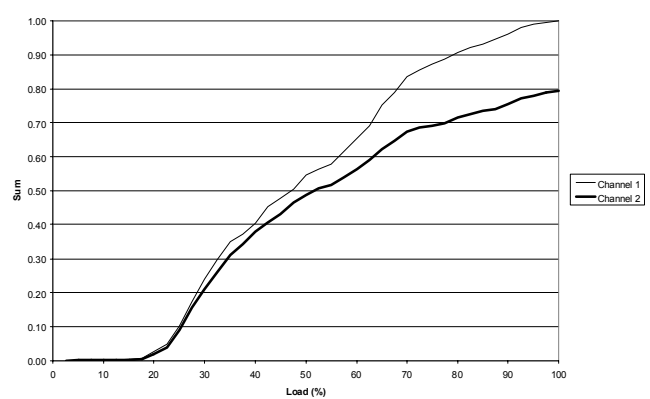

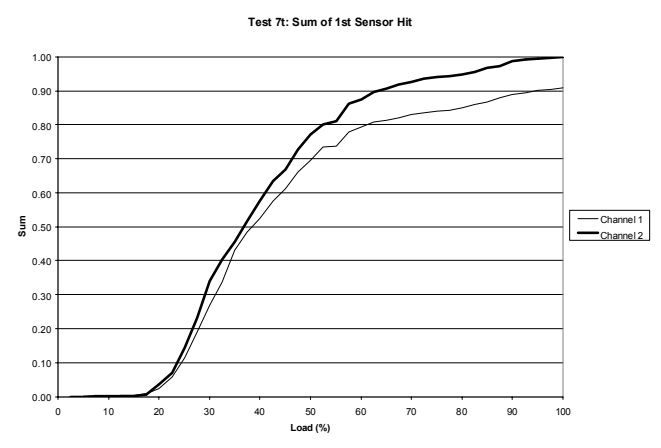

Figure 5.9 Sum of First Sensor Hit vs. Load (tests 1t-7t) 
Input Layer

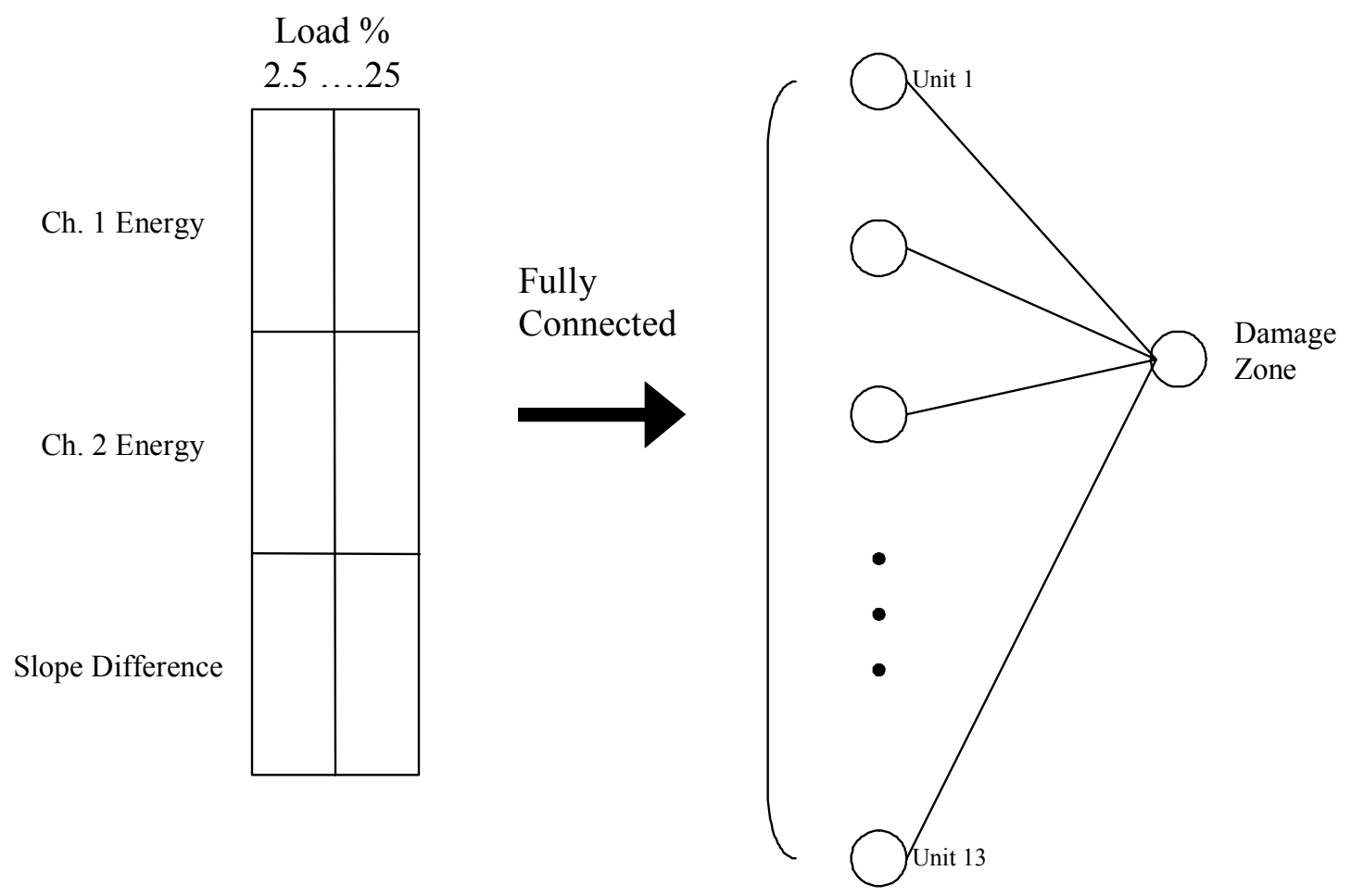

Figure 5.10 NN Architecture for Damage Zone Predictor 


\section{Chapter 6}

\section{Neural Network Analysis of Bending Specimens}

\subsection{Equipment}

For the Neural Network analysis of the bending specimens, the same computer program was used as with the tension specimens. Many of the same methods for data preparation used as with the tension specimens were also used for the evaluation of the bending specimens.

\subsubsection{Input Matrices}

The input files for the loading quarter predictor for the bending specimens were developed in the same way as those for the tension specimens. Figure 5.1 from Chapter 5 shows an example of the input matrix file. The one difference is the total number of matrices in the file. There were two sets of input files, the first with 14 or 16 matrices depending on the test. The second had 54 or 56 depending on the test. The total number of matrices available was 58 . This odd number was caused by the fact that test B1 was only loaded to slightly more than $50 \%$ of the expected failure load. This resulted in only two matrices from test B1 instead of the normal four.

\subsection{Loading Quarter Prediction}

As with the tension specimens, the $\mathrm{NN}$ analysis was split into two parts, load predictor and damage zone predictor. This prevented the interference of the load quarter predictor data in the prediction of the damage zone. Each of the matrices have a dimension of 10 rows by 10 columns ( 5 parameters for each of two sensors). The training sets and test sets were composed for the bending specimens as follows: 


\subsubsection{Training Set Matrices}

The same parameters as those used for the tension specimens were chosen for the bending tests analysis. This parameter information was used from just sensors one and two. Signals of $60 \mathrm{~dB}$ were kept in the bending test trials. The average counts parameter for all fifteen tests can be seen in Figures 6.1-6.3. Figures 6.4-6.6 display the average energy parameter for all fifteen tests. Figures 6.7-6.9 and 6.10-6.12 are the plots for the average duration and average amplitude parameters respectively. All of these graphs were displayed in order to show the strong patterns that each parameter displays. These are the patterns that the NN system uses to establish connections to the results. A particularly strong pattern can be seen in the frequency hits parameter plots. The graphs for the number of hits $>69 \mathrm{~dB}$ that are between $100 \mathrm{kHz}$ and $400 \mathrm{kHz}$ are shown in Figures 6.13-6.15. As with the tension tests, each training set consisted of the information from all but one of the tests. Two sets of fifteen training sets were constructed. The first was a set that consisted of the specimens separated by group. This resulted in each training set having the results from four tests in it. The second set of training sets consisted of all fifteen tests combined. This resulted in training sets with the results from fourteen specimens in them. As with the tension tests, each parameter was normalized for each specimen separately in order to magnify each pattern. Table 6.1 shows the maximum and minimum values of each parameter for each specimen.

\subsubsection{Test Set Matrices}

A total of 30 trials were conducted for the bending tests, the first fifteen for the three groups separated and the second fifteen for all groups together. As with the tension tests, the test 
set was the data from the one specimen that was not used in the training set. An example of this for the first fifteen is as follows. For trial B1, specimens B2-B5 composed the training set and test B1 was used for the test set. For trial B1 in the second set of fifteen trials, test B2-B10 \& C1-C5 made up the training set and test B1 was the test set. These methods were repeated for trials in the first and second set of fifteen. Once again the 40-20-40 rule was used for correct rate.

\subsubsection{Results}

A schematic of the NN architecture can be seen in Figure 5.8 of Chapter 5. The one exception is that the number of hidden units used is 6 instead of 13 . Table 6.2 shows the results of the NN analysis of the specimens when they were separated into three separate sets. This set of trials resulted in a total CR of $60.36 \%$. As can be seen, some quarters had fairly low CRs $\left(2^{\text {nd }}\right.$ $=40 \%$ ). Of particular interest is the CR of $71.43 \%$ in the $4^{\text {th }}$ quarter. This indicates that the predictor gets more accurate as the results become more critical (near to failure). Table 6.3 is a summary of the results for the second set of trials. This is set in which all groups were combined into one set. The total CR for these trials was $53.33 \%$. As with the previous trial, the prediction becomes more accurate in the $4^{\text {th }}$ quarter when it is more crucial. The CR for that quarter was $78.57 \%$.

These results show that data from different types/brands of specimens can be combined and still produce effective $\mathrm{NN}$ results for load prediction. The overall $\mathrm{CR}$ for all specimens combined is lower, but in the case of the $4^{\text {th }}$ quarter, combining the specimens actually resulted in a more accurate result. However, it may be found that as the number of samples of each type 
of specimen (only 5 of each in this case) is increased, the case of each type of specimen being separate may become more accurate.

\subsection{Damage Zone Prediction}

As with the tension specimens, damage zone was found to be more appropriate than location due to the difficulty of locating a definite spot of final breakage in FRP materials. As was done before, a value from 0 to 2 was predicted with 0 corresponding to the area around sensor 1 and 2 the area around sensor 2. It was found that tests B6 - B10 (group 3) displayed abnormal patterns. In some cases, the data and visual results point to two different damage zones. This problem is attributed to delamination failures of these specimens. The delaminations occur throughout the area being monitored by sensors 1 and 2 . This causes the sum of first sensor hit parameter used for prediction of damage zone to often be misleading. Due to these abnormalities, only tests B1-B5 and C1-C5 were used for damage zone prediction of the bending specimens.

\subsubsection{Training Set Matrices}

As with the loading quarter predictor, two sets of training sets were constructed. Once again, the first set was each of the two types of specimens separated with the second set consisting of all ten tests combined (due to elimination of tests B6-B10). Each matrix's dimensions were 3 rows by 10 columns. The parameters used for damage zone prediction were average energy for each of the two sensors, which can be seen in Figures 6.4 - 6.6, along with the slope difference of the sum of first sensor hit curves. The sum of first sensor hit curves were developed from only locatable signals. These curves for tests B1-B5 (group 1) and C1-C5 (group 2) are seen in Figures 6.16 and 6.17 respectively. Care was taken to use the correct 
wavespeed when finding the maximum time difference between locatable signals due to the fact that all three types of specimens had unique wavespeeds. The wavespeed for specimens B1-B5 (Bedford Composites deck pieces, group 1) was found to be 147,000 in/s. A wavespeed of 125,000 in/s was found for tests B6-B10 (Bedford Composites plate pieces, group 3) and 136,000 in/s for tests C1-C5 (Creative Pultrusions deck pieces, group 2). Due to material inhomogeneity and inconsistencies with the pencil break strike, an average of several pencil breaks was used in order to get a more accurate wavespeed value. These wavespeeds are summarized in Table 6.4 .

\subsubsection{Test Set Matrices}

Once again, each specimen's data was used as a test set while the data for the other sets was used for the training set. Correct rate was determined by the 40-20-40 rule.

\subsubsection{Results}

Figure 5.10 from Chapter 5 shows a schematic of the damage zone predictor architecture. Instead of 13 hidden units, (as with the tension specimens) 6 hidden units were used for the analysis of the bending specimens. Table 6.5 is a summary of the results of the damage zone predictor for the set of trials when the data was separated by group. These trials resulted in a CR of $67.78 \%$ with the $1^{\text {st }}$ quarter (essentially no damage) having the best CR at $100 \%$. The second set of damage zone prediction trials (all specimens together) is shown in Table 6.6. It can be seen that the total resulting CR is 61.67 with the first quarter once again having a CR of $100 \%$. Near the end of the loading, the accuracy of the predictor is decreased greatly. This is due to the fact that the specimen is seeing damage throughout, instead of one concentrated location. This is 
more than likely negligible due to the fact that by the time the structure reaches this point, it is too far gone to be saved anyway.

As with the loading predictor, the damage zone predictor's results for the specimens separated by group show higher CRs. The results for the groups combined do in fact show prediction rates of over $60 \%$. The CRs for both of these cases may also be increased by the addition of larger amounts of tested specimens. As with the damage zone predictor for the tension tests, the predictor for the bending tests is much less accurate in the last quarter. This is due to the fact that at that point, the breakage begins to spread throughout the specimen. Another factor in this is the fact that more cracks appear near the end. This changes the material attenuation and decreases the wavespeed through the specimen, which sometimes results in actual signals being eliminated as noise. 


\begin{tabular}{|c|c|c|c|c|c|c|c|c|c|c|}
\hline \multirow[t]{3}{*}{ Parameter } & \multicolumn{10}{|c|}{ Training Sets } \\
\hline & \multicolumn{2}{|c|}{$\mathrm{B} 1$} & \multicolumn{2}{|c|}{$\mathrm{B} 2$} & \multicolumn{2}{|c|}{ B3 } & \multicolumn{2}{|c|}{$\mathrm{B} 4$} & \multicolumn{2}{|c|}{$\mathrm{B} 5$} \\
\hline & $\min$ & $\max$ & $\min$ & $\max$ & $\min$ & $\max$ & $\min$ & $\max$ & $\min$ & $\max$ \\
\hline Counts & 7.94 & 30.25 & 11.00 & 28.40 & 6.75 & 30.00 & 7.00 & 31.50 & 7.00 & 31.11 \\
\hline Energy (energy counts) & 5.44 & 19.35 & 5.67 & 17.60 & 5.58 & 20.00 & 5.00 & 19.80 & 5.50 & 20.00 \\
\hline Duration ( $\mu$ seconds) & 19.94 & 114.33 & 29.00 & 117.33 & 17.75 & 120.00 & 19.00 & 100.00 & 14.00 & 120.00 \\
\hline Amplitude (dB) & 61.00 & 72.00 & 61.00 & 70.00 & 61.00 & 72.00 & 61.00 & 72.00 & 61.00 & 74.00 \\
\hline \multirow[t]{3}{*}{ Frequency (hits) } & 1.00 & 58.00 & 1.00 & 21.00 & 1.00 & 101.00 & 1.00 & 61.00 & 1.00 & 40.00 \\
\hline & \multicolumn{2}{|c|}{$\mathrm{B} 6$} & \multicolumn{2}{|c|}{ B7 } & \multicolumn{2}{|c|}{$\mathrm{B} 8$} & \multicolumn{2}{|c|}{$\mathrm{B} 9$} & \multicolumn{2}{|c|}{$\mathrm{B} 10$} \\
\hline & $\min$ & $\max$ & $\min$ & $\max$ & $\min$ & $\max$ & $\min$ & $\max$ & $\min$ & $\max$ \\
\hline Counts & 10.00 & 28.57 & 8.00 & 29.97 & 8.00 & 27.61 & 5.67 & 36.36 & 9.00 & 24.43 \\
\hline Energy (energy counts) & 7.00 & 17.43 & 5.00 & 24.10 & 5.29 & 21.53 & 5.00 & 35.96 & 5.00 & 18.45 \\
\hline Duration ( $\mu$ seconds) & 31.00 & 94.50 & 28.00 & 133.97 & 24.33 & 137.00 & 20.33 & 192.80 & 25.50 & 118.75 \\
\hline Amplitude (dB) & 61.33 & 70.71 & 62.33 & 74.07 & 61.43 & 72.00 & 61.43 & 77.68 & 61.00 & 72.55 \\
\hline \multirow[t]{3}{*}{ Frequency (hits) } & 1.00 & 183.00 & 1.00 & 144.00 & 1.00 & 179.00 & 1.00 & 223.00 & 1.00 & 235.00 \\
\hline & \multicolumn{2}{|c|}{$\mathrm{C} 1$} & \multicolumn{2}{|c|}{$\mathrm{C} 2$} & \multicolumn{2}{|c|}{$\mathrm{C} 3$} & \multicolumn{2}{|c|}{$\mathrm{C4}$} & \multicolumn{2}{|c|}{$\mathrm{C5}$} \\
\hline & $\min$ & $\max$ & $\min$ & $\max$ & $\min$ & $\max$ & $\min$ & $\max$ & $\min$ & $\max$ \\
\hline Counts & 10.00 & 37.67 & 10.00 & 34.67 & 13.50 & 42.33 & 17.00 & 54.00 & 10.60 & 34.00 \\
\hline Energy (energy counts) & 6.00 & 22.50 & 6.00 & 19.50 & 4.75 & 30.00 & 5.50 & 50.78 & 5.75 & 21.28 \\
\hline Duration ( $\mu$ seconds) & 31.00 & 135.71 & 33.00 & 156.67 & 39.25 & 141.37 & 44.00 & 200.00 & 38.20 & 141.96 \\
\hline Amplitude $(\mathrm{dB})$ & 61.00 & 72.33 & 61.33 & 72.00 & 61.00 & 74.00 & 61.50 & 80.25 & 60.50 & 70.09 \\
\hline Frequency (hits) & 1.00 & 189.00 & 1.00 & 155.00 & 1.00 & 262.00 & 1.00 & 150.00 & 1.00 & 264.00 \\
\hline
\end{tabular}

Table 6.1 Minimum/Maximum Values for Normalization 


\section{Groups Separate, 6 Hidden Units}

\begin{tabular}{|c|c|c|}
\hline \multicolumn{1}{|c|}{} & \multicolumn{1}{c}{ B1 } & \\
\hline known & predicted & difference \\
\hline 1 & 1.13 & 0.13 \\
\hline 2 & 1.81 & -0.19 \\
\hline 3 & - & - \\
\hline 4 & - & - \\
\hline CR & 100 & \multicolumn{1}{|c}{} \\
\cline { 1 - 2 } & \multicolumn{2}{|l}{}
\end{tabular}

\begin{tabular}{|c|c|c|}
\hline \multicolumn{1}{|c|}{} & B6 & \\
\hline known & predicted & difference \\
\hline 1 & 1.93 & 0.93 \\
\hline 2 & 2.36 & 0.36 \\
\hline 3 & 2.16 & -0.84 \\
\hline 4 & 3.82 & -0.18 \\
\hline CR & 50 & \multicolumn{1}{|c}{} \\
& &
\end{tabular}

\begin{tabular}{|c|c|c|}
\hline \multicolumn{3}{|c|}{01} \\
\hline known & predicted & difference \\
\hline 1 & 1.07 & 0.07 \\
\hline 2 & 2.28 & 0.28 \\
\hline 3 & 3.55 & 0.55 \\
\hline 4 & 3.51 & -0.49 \\
\hline$\overline{C R}$ & 50 & \\
\hline
\end{tabular}

\begin{tabular}{|c|c|c|}
\hline \multicolumn{3}{|c|}{ B2 } \\
\hline known & predicted & difference \\
\hline 1 & 1.11 & 0.11 \\
\hline 2 & 2.21 & 0.21 \\
\hline 3 & 3.59 & 0.59 \\
\hline 4 & 3.74 & -0.26 \\
\hline CR & 75 & \\
\hline
\end{tabular}

\begin{tabular}{|c|c|c|}
\hline \multicolumn{3}{|c|}{$\mathrm{B7}$} \\
\hline known & predicted & difference \\
\hline 1 & 1.3 & 0.30 \\
\hline 2 & 2.86 & 0.86 \\
\hline 3 & 2.6 & -0.40 \\
\hline 4 & 3.69 & -0.31 \\
\hline CR & 50 & \\
\hline
\end{tabular}

\begin{tabular}{|c|c|c|}
\hline \multicolumn{3}{|c|}{$\mathbb{C 2}$} \\
\hline known & predicted & difference \\
\hline 1 & 0.94 & -0.06 \\
\hline 2 & 2.4 & 0.40 \\
\hline 3 & 2.97 & -0.03 \\
\hline 4 & 3.83 & -0.17 \\
\hline CR & 75 & \\
\hline
\end{tabular}

\begin{tabular}{|c|c|c|}
\hline \multicolumn{3}{|c|}{$B 3$} \\
\hline known & predicted & difference \\
\hline 1 & 0.96 & -0.04 \\
\hline 2 & 2.51 & 0.51 \\
\hline 3 & 3.52 & 0.52 \\
\hline 4 & 3.63 & -0.37 \\
\hline CR & 50 & \\
\hline
\end{tabular}

\begin{tabular}{|c|c|c|}
\hline \multicolumn{3}{|c|}{ B8 } \\
\hline known & predicted & difference \\
\hline 1 & 3.09 & 2.09 \\
\hline 2 & 2.93 & 0.93 \\
\hline 3 & 3.21 & 0.21 \\
\hline 4 & 3.76 & -0.24 \\
\hline CR & 50 & \\
\hline
\end{tabular}

\begin{tabular}{|c|c|c|}
\hline known & predicted & difference \\
\hline 1 & 1.01 & 0.01 \\
\hline 2 & 1.98 & -0.02 \\
\hline 3 & 2.96 & -0.04 \\
\hline 4 & 2.88 & -1.12 \\
\hline CR & 75 & \multicolumn{1}{|c}{} \\
\cline { 1 - 2 } & \multicolumn{2}{|c}{}
\end{tabular}

\begin{tabular}{|c|c|c|}
\hline \multirow{2}{*}{} & \multicolumn{1}{c}{ B4 } & \\
\hline known & predicted & difference \\
\hline 1 & 1.05 & 0.05 \\
\hline 2 & 2.06 & 0.06 \\
\hline 3 & 3.13 & 0.13 \\
\hline 4 & 2.73 & -1.27 \\
\hline CR & 75 & \multicolumn{1}{|c}{} \\
\cline { 1 - 1 } & \multicolumn{2}{|c}{}
\end{tabular}

\begin{tabular}{|c|c|c|}
\hline \multicolumn{3}{|c|}{$\mathrm{B} 9$} \\
\hline known & predicted & difference \\
\hline 1 & 0.75 & -0.25 \\
\hline 2 & 1.33 & -0.67 \\
\hline 3 & 2.73 & -0.27 \\
\hline 4 & 3.73 & -0.27 \\
\hline CR & 75 & \\
\hline
\end{tabular}

\begin{tabular}{|c|c|c|}
\hline \multirow{2}{*}{} & \multicolumn{1}{c}{$\mathrm{C} 4$} & \\
\hline known & predicted & difference \\
\hline 1 & 1.3 & 0.30 \\
\hline 2 & 2.47 & 0.47 \\
\hline 3 & 2.41 & -0.59 \\
\hline 4 & 3.66 & -0.34 \\
\hline CR & 50 & \multicolumn{1}{|c}{} \\
\cline { 1 - 2 } & &
\end{tabular}

\begin{tabular}{|c|c|c|}
\hline \multicolumn{3}{|c|}{ B5 } \\
\hline known & predicted & difference \\
\hline 1 & 0.81 & -0.19 \\
\hline 2 & 2.45 & 0.45 \\
\hline 3 & 2.74 & -0.26 \\
\hline 4 & 3.14 & -0.86 \\
\hline CR & 50 & \\
\hline
\end{tabular}

\begin{tabular}{|c|c|c|}
\hline \multicolumn{2}{|c|}{ B 10 } & predicted \\
\hline known & difference \\
\hline 1 & 2.12 & 1.12 \\
\hline 2 & 2.68 & 0.68 \\
\hline 3 & 3.11 & 0.11 \\
\hline 4 & 3.83 & -0.17 \\
\hline CR & 50 & \multicolumn{1}{|c}{} \\
\cline { 1 - 2 } & \multicolumn{2}{|c}{}
\end{tabular}

\begin{tabular}{|c|c|c|}
\hline \multicolumn{3}{|c|}{$c 5$} \\
\hline known & predicted & difference \\
\hline 1 & 1.18 & 0.18 \\
\hline 2 & 1.58 & -0.42 \\
\hline 3 & 3.44 & 0.44 \\
\hline 4 & 3.77 & -0.23 \\
\hline CR & 50 & \\
\hline
\end{tabular}

\section{Summary}

\begin{tabular}{|c|c|}
\hline \hline Quarter & CR \\
\hline \hline 1.00 & 80.00 \\
\hline 2.00 & 40.00 \\
\hline 3.00 & 50.00 \\
\hline 4.00 & 71.43 \\
\hline Total & 60.36 \\
\hline
\end{tabular}

Table 6.2 Load Quarter Predictor Results, Groups Separated 


\section{All Groups Combined, 6 Hidden Units}

\begin{tabular}{|c|c|c|}
\hline \multirow{2}{*}{ B1 } \\
\hline known & predicted & difference \\
\hline 1 & 1.52 & 0.52 \\
\hline 2 & 1.87 & -0.13 \\
\hline 3 & - & - \\
\hline 4 & - & - \\
\hline CR & 50 & \multicolumn{1}{|c}{} \\
\cline { 1 - 2 } & \multicolumn{2}{|c}{} \\
\cline { 1 - 2 } & &
\end{tabular}

\begin{tabular}{|c|c|c|}
\hline \multicolumn{3}{|c|}{$\overline{B 6}$} \\
\hline known & predicted & difference \\
\hline 1 & 0.82 & -0.18 \\
\hline 2 & 2.69 & 0.69 \\
\hline 3 & 1.79 & -1.21 \\
\hline 4 & 3.37 & -0.63 \\
\hline CR & 25 & \\
\hline
\end{tabular}

\begin{tabular}{|c|c|c|}
\hline \multicolumn{3}{|c|}{$\mathrm{Cl}$} \\
\hline known & predicted & difference \\
\hline 1 & 0.75 & -0.25 \\
\hline 2 & 2.09 & 0.09 \\
\hline 3 & 3.82 & 0.82 \\
\hline 4 & 3.69 & -0.31 \\
\hline CR & 75 & \\
\hline
\end{tabular}

\begin{tabular}{|c|c|c|}
\hline \multirow{2}{*}{} & B2 2 & \\
\hline known & predicted & difference \\
\hline 1 & 1.25 & 0.25 \\
\hline 2 & 2.06 & 0.06 \\
\hline 3 & 3.83 & 0.83 \\
\hline 4 & 3.8 & -0.20 \\
\hline CR & 75 & \multicolumn{1}{|c}{} \\
\cline { 1 - 2 } & \multicolumn{2}{|c}{} \\
\cline { 2 - 3 } & &
\end{tabular}

\begin{tabular}{|c|c|c|}
\hline \multicolumn{3}{|c|}{ B7\% } \\
\hline known & predicted & difference \\
\hline 1 & 1.46 & 0.46 \\
\hline 2 & 2.37 & 0.37 \\
\hline 3 & 2.28 & -0.72 \\
\hline 4 & 3.86 & -0.14 \\
\hline CR & 50 & \\
\hline
\end{tabular}

\begin{tabular}{|c|c|c|}
\hline \multicolumn{1}{|c|}{} & $\mathbf{C 2}$ & \\
\hline known & predicted & difference \\
\hline 1 & 0.9 & -0.10 \\
\hline 2 & 3.15 & 1.15 \\
\hline 3 & 3.71 & 0.71 \\
\hline 4 & 3.95 & -0.05 \\
\hline CR & 50 & \multicolumn{1}{|c}{} \\
\cline { 1 - 2 } & &
\end{tabular}

\begin{tabular}{|c|c|c|}
\hline & \multicolumn{1}{|c|}{ B 3 } & \\
\hline known & predicted & difference \\
\hline 1 & 1.27 & 0.27 \\
\hline 2 & 2.61 & 0.61 \\
\hline 3 & 3.73 & 0.73 \\
\hline 4 & 3.41 & -0.59 \\
\hline CR & 25 & \multicolumn{1}{|c}{} \\
\cline { 1 - 2 } & \multicolumn{2}{|c}{}
\end{tabular}

\begin{tabular}{|c|c|c|}
\hline & \multicolumn{1}{|c|}{ B 8} & \\
\hline known & predicted & difference \\
\hline 1 & 3.36 & 2.36 \\
\hline 2 & 2.48 & 0.48 \\
\hline 3 & 2.98 & -0.02 \\
\hline 4 & 3.83 & -0.17 \\
\hline CR & 50 & \multicolumn{1}{|c}{} \\
\cline { 1 - 2 } & \multicolumn{2}{|c}{}
\end{tabular}

Q3

\begin{tabular}{|c|c|c|}
\hline \multicolumn{3}{|c|}{ C3: } \\
\hline known & predicted & difference \\
\hline 1 & 0.58 & -0.42 \\
\hline 2 & 2.46 & 0.46 \\
\hline 3 & 3.16 & 0.16 \\
\hline 4 & 3.77 & -0.23 \\
\hline CR & 50 & \\
\hline
\end{tabular}

\begin{tabular}{|c|c|c|}
\hline \multicolumn{3}{|c|}{ B4 } \\
\hline known & predicted & difference \\
\hline 1 & 1.09 & 0.09 \\
\hline 2 & 2.36 & 0.36 \\
\hline 3 & 3.59 & 0.59 \\
\hline 4 & 3.73 & -0.27 \\
\hline $\mathrm{CR}$ & 75 & \\
\hline
\end{tabular}

\begin{tabular}{|c|c|c|}
\hline \multicolumn{3}{|c|}{$\mathrm{B9}$} \\
\hline known & predicted & difference \\
\hline 1 & 0.88 & -0.12 \\
\hline 2 & 1.23 & -0.77 \\
\hline 3 & 2.05 & -0.95 \\
\hline 4 & 3.81 & -0.19 \\
\hline CR & 50 & \\
\hline
\end{tabular}

\begin{tabular}{|c|c|c|}
\hline \multicolumn{3}{|c|}{ C4: } \\
\hline known & predicted & difference \\
\hline 1 & 0.61 & -0.39 \\
\hline 2 & 1.9 & -0.10 \\
\hline 3 & 3.42 & 0.42 \\
\hline 4 & 3.91 & -0.09 \\
\hline $\mathrm{CR}$ & 75 & \\
\hline
\end{tabular}

\begin{tabular}{|c|c|c|}
\hline \multicolumn{3}{|c|}{ B5 } \\
\hline known & predicted & difference \\
\hline 1 & 0.87 & -0.13 \\
\hline 2 & 2.58 & 0.58 \\
\hline 3 & 3.05 & 0.05 \\
\hline 4 & 2.51 & -1.49 \\
\hline CR & 50 & \\
\hline
\end{tabular}

\begin{tabular}{|c|c|c|}
\hline \multicolumn{3}{|c|}{$B 10$} \\
\hline known & predicted & difference \\
\hline 1 & 1.43 & 0.43 \\
\hline 2 & 2.02 & 0.02 \\
\hline 3 & 2.32 & -0.68 \\
\hline 4 & 3.64 & -0.36 \\
\hline CR & 50 & \\
\hline
\end{tabular}

\begin{tabular}{|c|c|c|}
\hline \multirow{4}{*}{ C5 } & \\
\hline known & predicted & difference \\
\hline 1 & 0.78 & -0.22 \\
\hline 2 & 2.55 & 0.55 \\
\hline 3 & 3.69 & 0.69 \\
\hline 4 & 3.77 & -0.23 \\
\hline CR & 50 & \multicolumn{1}{|c}{} \\
\cline { 2 - 2 } & \multicolumn{2}{|c}{}
\end{tabular}

\begin{tabular}{|c|c|}
\hline \multicolumn{2}{|c|}{ Summary } \\
\hline$\overline{\text { Quarter }}$ & $\overline{C R}$ \\
\hline 1.00 & 66.67 \\
\hline 2.00 & 46.67 \\
\hline 3.00 & 21.43 \\
\hline 4.00 & 78.57 \\
\hline Total & 53.33 \\
\hline
\end{tabular}

Table 6.3 Load Quarter Predictor Results, All Groups Combined 


\begin{tabular}{|c|c|c|c|c|c|c|c|c|}
\hline \multicolumn{9}{|c|}{ Wavespeed Calculation } \\
\hline \multicolumn{3}{|c|}{ Creative Pultrisions Deck Section } & \multicolumn{3}{|c|}{ Bedford Deck Section } & \multicolumn{3}{|c|}{ Bedford Plate Section } \\
\hline$\Delta t$ & $\Delta d$ & $\mathrm{v}$ & $\Delta t$ & $\Delta d$ & V & $\Delta \mathrm{t}$ & $\Delta d$ & V \\
\hline (useconds) & (inches) & (in/s) & ( $\mu$ seconds) & (inches) & (in/s) & (useconds) & (inches) & (in/s) \\
\hline 19 & 2.67 & 142781 & 19 & 2.67 & 144324 & 21 & 2.67 & 127143 \\
\hline 20 & 2.67 & 136224 & 17 & 2.67 & 154335 & 21 & 2.67 & 124766 \\
\hline 21 & 2.67 & 128365 & 18 & 2.67 & 152571 & 21 & 2.67 & 128365 \\
\hline 21 & 2.67 & 128986 & 19 & 2.67 & 140526 & 22 & 2.67 & 120270 \\
\hline 19 & 2.67 & 138342 & 19 & 2.67 & 144324 & 21 & 2.67 & 128986 \\
\hline 19 & 2.67 & 142021 & 18 & 2.67 & 151705 & 21 & 2.67 & 127143 \\
\hline 19 & 2.67 & 140526 & 19 & 2.67 & 142021 & 22 & 2.67 & 123041 \\
\hline 21 & 2.67 & 127143 & 18 & 2.67 & 146703 & 21 & 2.67 & 127143 \\
\hline 20 & 2.67 & 136224 & 19 & 2.67 & 140526 & 22 & 2.67 & 121364 \\
\hline 19 & 2.67 & 140526 & 18 & 2.67 & 146703 & 22 & 2.67 & 121364 \\
\hline 19 & 2.67 & 138342 & 18 & 2.67 & 145109 & 21 & 2.67 & 127143 \\
\hline \multirow[t]{2}{*}{20} & 2.67 & 136923 & 17 & 2.67 & 155233 & 22 & 2.67 & 123611 \\
\hline & AVERAGE & 136400 & & AVERAGE & 147000 & & AVERAGE & 125000 \\
\hline
\end{tabular}

Table 6.4 Wave Speed Calculation Summary 


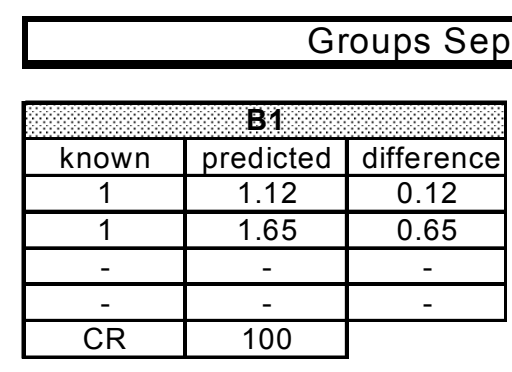

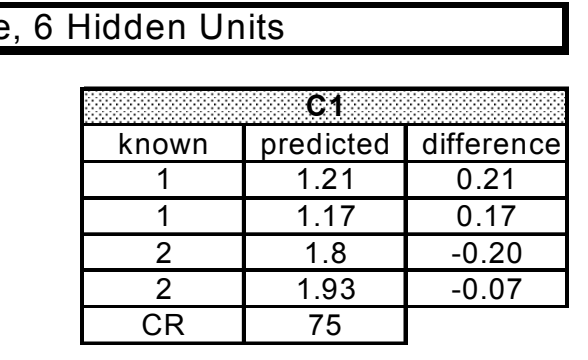

\begin{tabular}{|c|c|c|}
\hline \multicolumn{3}{|c|}{82} \\
\hline known & predicted & difference \\
\hline 1 & 1.05 & 0.05 \\
\hline 1 & 0.88 & -0.12 \\
\hline 0 & 1.01 & 1.01 \\
\hline 0 & 0.83 & 0.83 \\
\hline $\mathrm{CR}$ & 100 & \\
\hline
\end{tabular}

\begin{tabular}{|c|c|c|}
\hline known & predicted & difference \\
\hline 1 & 1.07 & 0.07 \\
\hline 1 & 1.66 & 0.66 \\
\hline 1 & 1.19 & 0.19 \\
\hline 1 & 0.4 & -0.60 \\
\hline CR & 50 & \multicolumn{1}{|c}{} \\
\cline { 1 - 2 } & \multicolumn{2}{|c}{} \\
\cline { 1 - 2 } & &
\end{tabular}

\begin{tabular}{|c|c|c|}
\hline \multicolumn{3}{|c|}{$\mathrm{B3}$} \\
\hline known & predicted & difference \\
\hline 1 & 1.16 & 0.16 \\
\hline 1 & 0.62 & -0.38 \\
\hline 1 & 1.34 & 0.34 \\
\hline 1 & 0.88 & -0.12 \\
\hline $\mathrm{CR}$ & 50 & \\
\hline
\end{tabular}

\begin{tabular}{|c|c|c|}
\hline \multicolumn{3}{|c|}{ - $\mathrm{C} 3 \mathrm{~B}$} \\
\hline known & predicted & difference \\
\hline 1 & 0.97 & -0.03 \\
\hline 1 & 0.45 & -0.55 \\
\hline 2 & 0.72 & -1.28 \\
\hline 2 & 1.81 & -0.19 \\
\hline $\mathrm{CR}$ & 25 & \\
\hline
\end{tabular}

\begin{tabular}{|c|c|c|}
\hline \multicolumn{3}{|c|}{$\mathrm{B} 4$} \\
\hline known & predicted & difference \\
\hline 1 & 0.97 & -0.03 \\
\hline 1 & 1.28 & 0.28 \\
\hline 1 & 1.81 & 0.81 \\
\hline 1 & 0.26 & -0.74 \\
\hline CR & 75 & \\
\hline
\end{tabular}

\begin{tabular}{|c|c|c|}
\hline \multicolumn{3}{|c|}{ Q4 } \\
\hline known & predicted & difference \\
\hline 1 & 1.05 & 0.05 \\
\hline 1 & 1.23 & 0.23 \\
\hline 2 & 0.96 & -1.04 \\
\hline 2 & 1.96 & -0.04 \\
\hline CR & 75 & \\
\hline
\end{tabular}

\begin{tabular}{|c|c|c|}
\hline \multicolumn{3}{|c|}{ B5 } \\
\hline known & predicted & difference \\
\hline 1 & 1.08 & 0.08 \\
\hline 1 & 0.6 & -0.40 \\
\hline 2 & 0.78 & -1.22 \\
\hline 2 & 0.51 & -1.49 \\
\hline CR & 50 & \\
\hline
\end{tabular}

\begin{tabular}{|c|c|c|}
\hline \multicolumn{3}{|c|}{65} \\
\hline known & predicted & difference \\
\hline 1 & 1.1 & 0.10 \\
\hline 1 & 1.1 & 0.10 \\
\hline 0 & 1.11 & 1.11 \\
\hline 0 & 1.14 & 1.14 \\
\hline $\mathrm{CR}$ & 50 & \\
\hline
\end{tabular}

\section{Summary}

\begin{tabular}{|c|c|}
\hline Quarter & CR \\
\hline \hline 1.00 & 100.00 \\
\hline 2.00 & 60.00 \\
\hline 3.00 & 44.44 \\
\hline 4.00 & 66.67 \\
\hline Total & 67.78 \\
\hline
\end{tabular}

Table 6.5 Damage Zone Predictor Results, Groups Separated 


\begin{tabular}{|c|c|c|}
\hline \multicolumn{3}{|c|}{ All Groups Com } \\
\begin{tabular}{|c|c|c|}
\hline \multicolumn{3}{|c|}{ B1 } \\
\hline known & predicted & difference \\
\hline 1 & 1.24 & 0.24 \\
\hline 1 & 1.35 & 0.35 \\
\hline- & - & - \\
\hline- & - & \\
\hline CR & 100 & \multicolumn{1}{|c}{} \\
\hline
\end{tabular}
\end{tabular}

\begin{tabular}{|c|c|c|}
\hline \multicolumn{3}{|c|}{$\mathrm{Cl}$} \\
\hline known & predicted & difference \\
\hline 1 & 1.17 & 0.17 \\
\hline 1 & 1.02 & 0.02 \\
\hline 2 & 1.83 & -0.17 \\
\hline 2 & 1.28 & -0.72 \\
\hline CR & 75 & \\
\hline
\end{tabular}

\begin{tabular}{|c|c|c|}
\hline \multirow{1}{*}{} & B2 & \\
\hline known & predicted & difference \\
\hline 1 & 1.02 & 0.02 \\
\hline 1 & 1.08 & 0.08 \\
\hline 0 & 0.32 & 0.32 \\
\hline 0 & 0.28 & 0.28 \\
\hline CR & 100 & \multicolumn{1}{|c}{} \\
\cline { 1 - 2 } & \multicolumn{2}{|c}{}
\end{tabular}

\begin{tabular}{|c|c|c|}
\hline \multicolumn{3}{|c|}{02} \\
\hline known & predicted & difference \\
\hline 1 & 1.11 & 0.11 \\
\hline 1 & 1.82 & 0.82 \\
\hline 1 & 0.67 & -0.33 \\
\hline 1 & 0.25 & -0.75 \\
\hline$\overline{C R}$ & 50 & \\
\hline
\end{tabular}

\begin{tabular}{|c|c|c|}
\hline \multirow{2}{*}{} & B $\mathbf{3}$ & \\
\hline known & predicted & difference \\
\hline 1 & 1.23 & 0.23 \\
\hline 1 & 1.33 & 0.33 \\
\hline 1 & 1.48 & 0.48 \\
\hline 1 & 1.7 & 0.70 \\
\hline CR & 50 & \multicolumn{1}{|c}{} \\
\cline { 1 - 2 } & \multicolumn{2}{|c}{}
\end{tabular}

\begin{tabular}{|c|c|c|}
\hline \multicolumn{3}{|c|}{$\mathbf{C 3}$} \\
\hline known & predicted & difference \\
\hline 1 & 1 & 0.00 \\
\hline 1 & 0.43 & -0.57 \\
\hline 2 & 1.15 & -0.85 \\
\hline 2 & 0.76 & -1.24 \\
\hline $\mathrm{CR}$ & 25 & \\
\hline
\end{tabular}

\begin{tabular}{|c|c|c|}
\hline \multirow{2}{*}{} & B 4 & \\
\hline known & predicted & difference \\
\hline 1 & 0.84 & -0.16 \\
\hline 1 & 1.34 & 0.34 \\
\hline 1 & 0.53 & -0.47 \\
\hline 1 & 0.26 & -0.74 \\
\hline CR & 75 & \multicolumn{1}{|c}{} \\
\cline { 1 - 2 } & \multicolumn{2}{|c}{}
\end{tabular}

\begin{tabular}{|c|c|c|}
\hline \multicolumn{3}{|c|}{ C4 } \\
\hline known & predicted & difference \\
\hline 1 & 0.99 & -0.01 \\
\hline 1 & 1.08 & 0.08 \\
\hline 2 & 0.65 & -1.35 \\
\hline 2 & 1.98 & -0.02 \\
\hline CR & 75 & \\
\hline
\end{tabular}

\begin{tabular}{|c|c|c|}
\hline \multicolumn{3}{|c|}{ B5 } \\
\hline known & predicted & difference \\
\hline 1 & 1 & 0.00 \\
\hline 1 & 0.8 & -0.20 \\
\hline 2 & 0.63 & -1.37 \\
\hline 2 & 0.8 & -1.20 \\
\hline CR & 50 & \\
\hline
\end{tabular}

\begin{tabular}{|c|c|c|}
\hline \multirow{2}{*}{} & C5 5 & \\
\hline known & predicted & difference \\
\hline 1 & 0.98 & -0.02 \\
\hline 1 & 0.77 & -0.23 \\
\hline 0 & 0.82 & 0.82 \\
\hline 0 & 0.79 & 0.79 \\
\hline CR & 50 & \multicolumn{1}{|c}{} \\
\cline { 1 - 2 } & \multicolumn{2}{|c}{} \\
\hline \multicolumn{2}{|c|}{}
\end{tabular}

\begin{tabular}{|c|c|}
\hline \multicolumn{2}{|c|}{ Summary } \\
\hline$\overline{\text { Quarter }}$ & $\overline{\mathrm{CR}}$ \\
\hline 1.00 & 100.00 \\
\hline 2.00 & 80.00 \\
\hline 3.00 & 33.33 \\
\hline 4.00 & 33.33 \\
\hline Total & 61.67 \\
\hline
\end{tabular}

Table 6.6 Damage Zone Predictor Results, All Groups Combined 

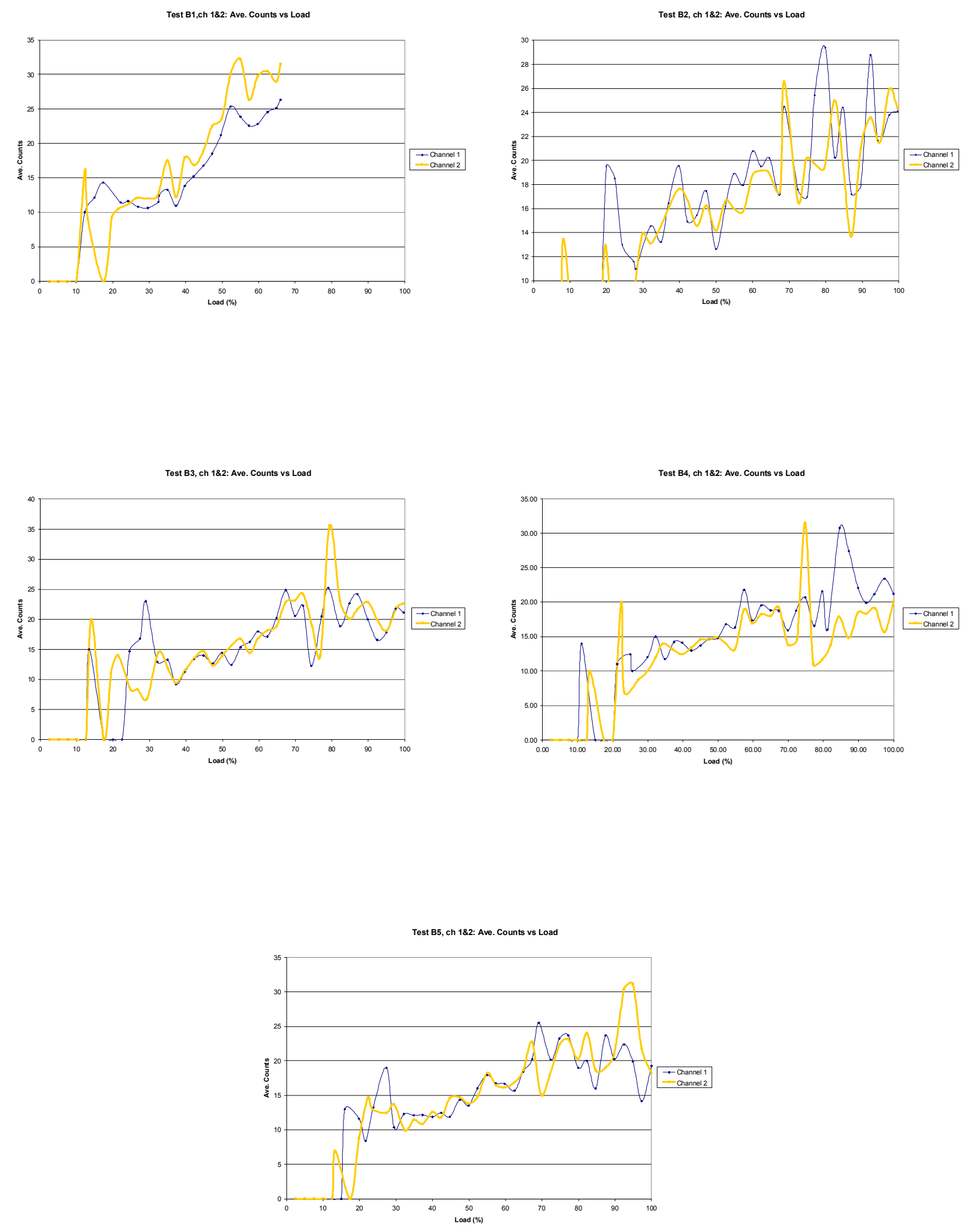

Figure 6.1 Average Counts vs. Load Parameter, Tests B1-B5 

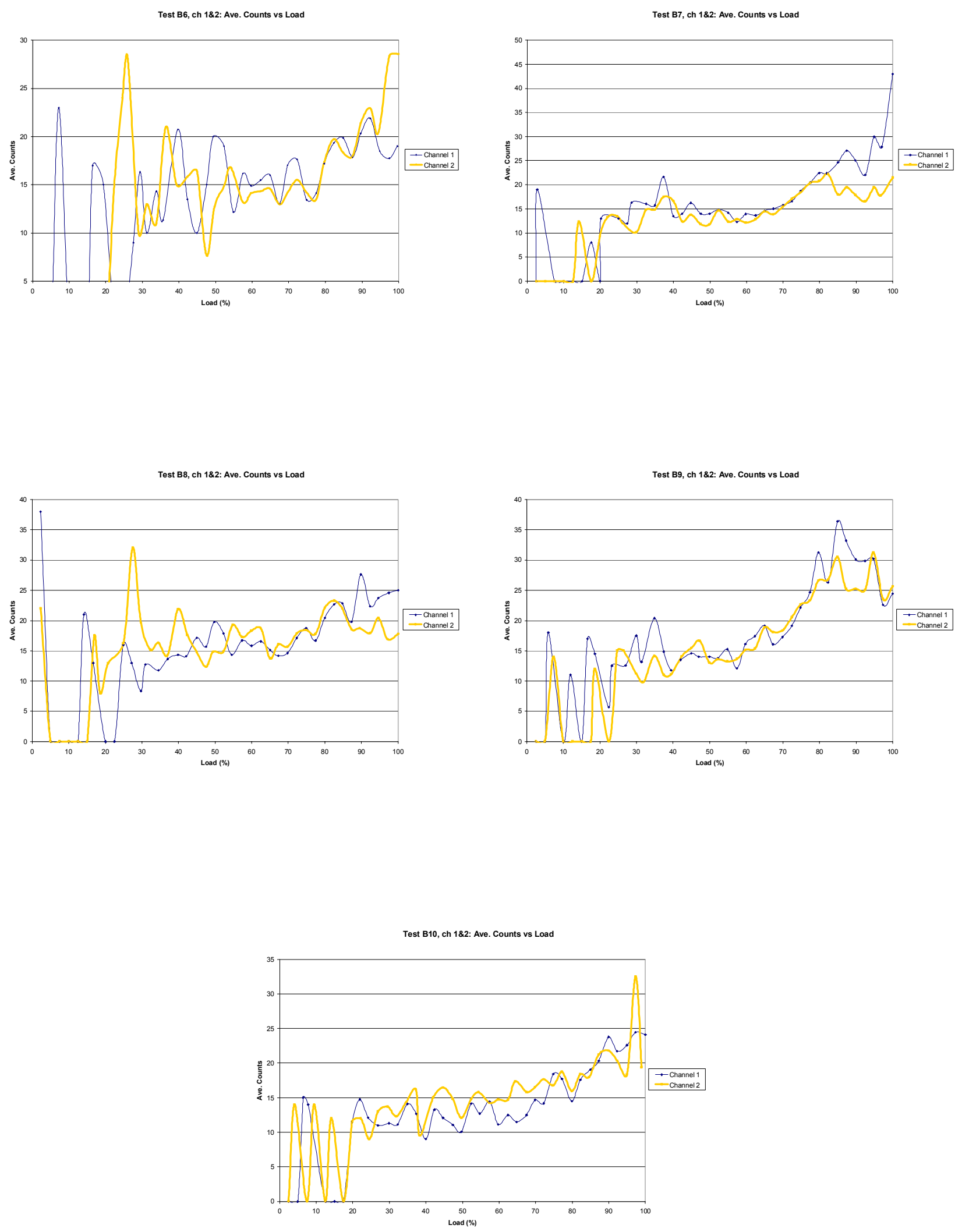

Figure 6.2 Average Counts vs. Load Parameter, Tests B6-B10 

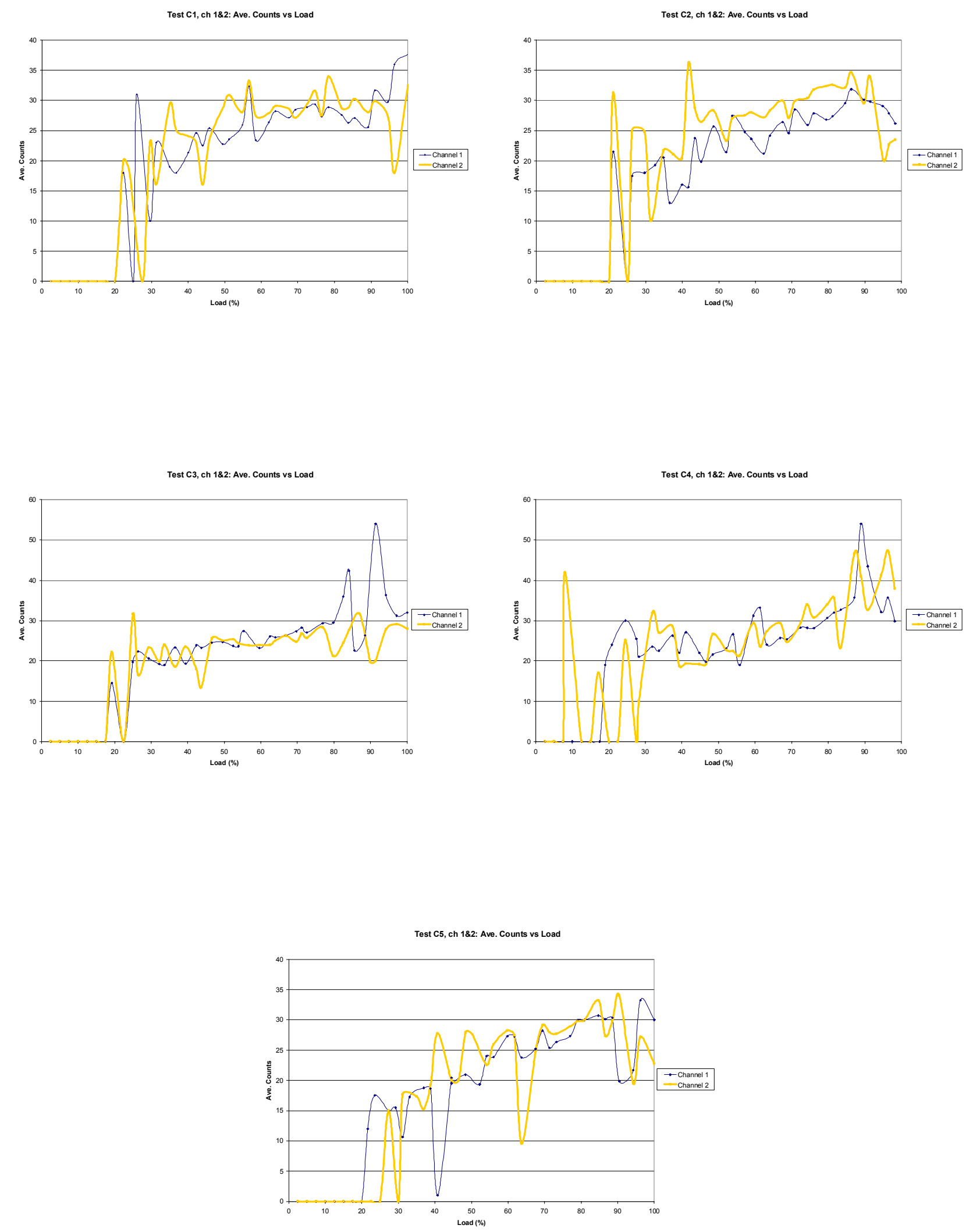

Figure 6.3 Average Counts vs. Load Parameter, Tests C1-C5 
Test B1, ch 182: Ave. Energy vs Load

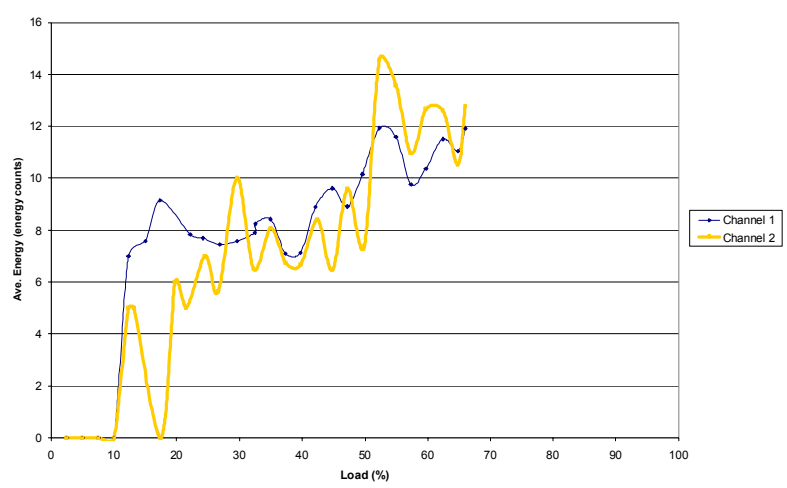

Test B3, ch 1\&2: Ave. Energy vs Load

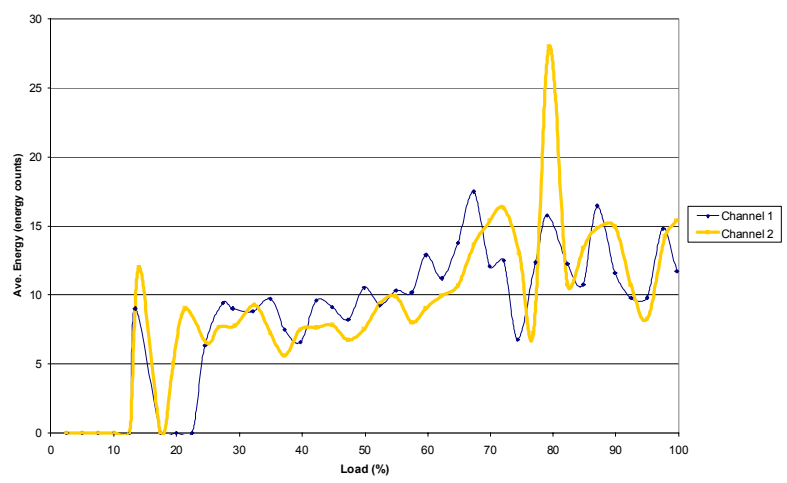

Test B2, ch 182: Ave, Energy vs Load

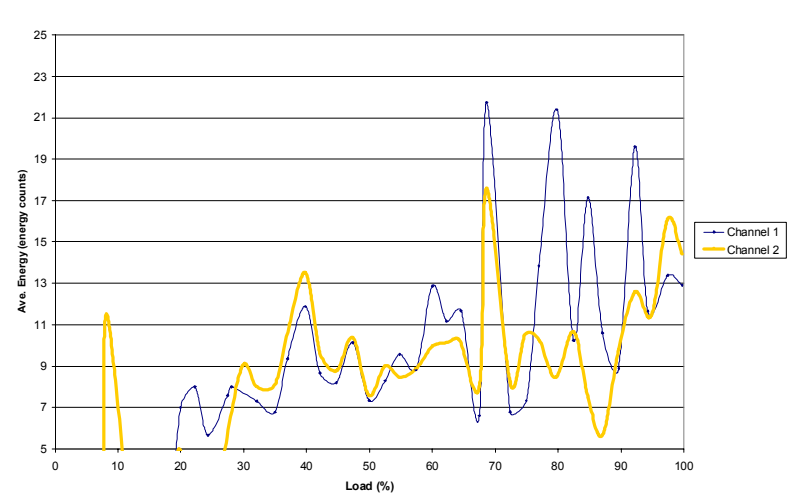

Test B4, ch 182: Ave. Energy vs Load

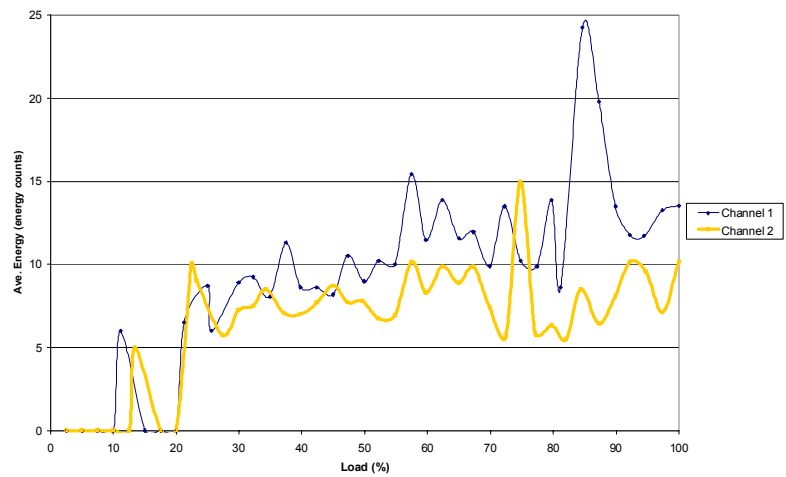

Test B5, ch 1\&2: Ave. Energy vs Load

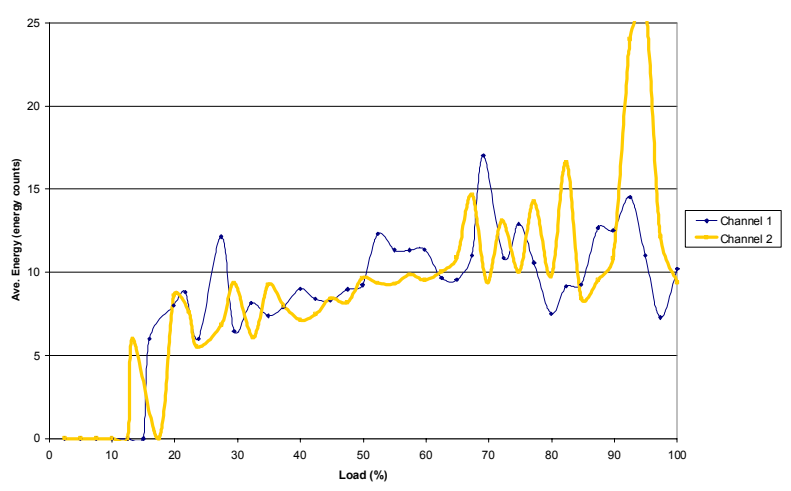

Figure 6.4 Average Energy vs. Load Parameter, Tests B1-B5 

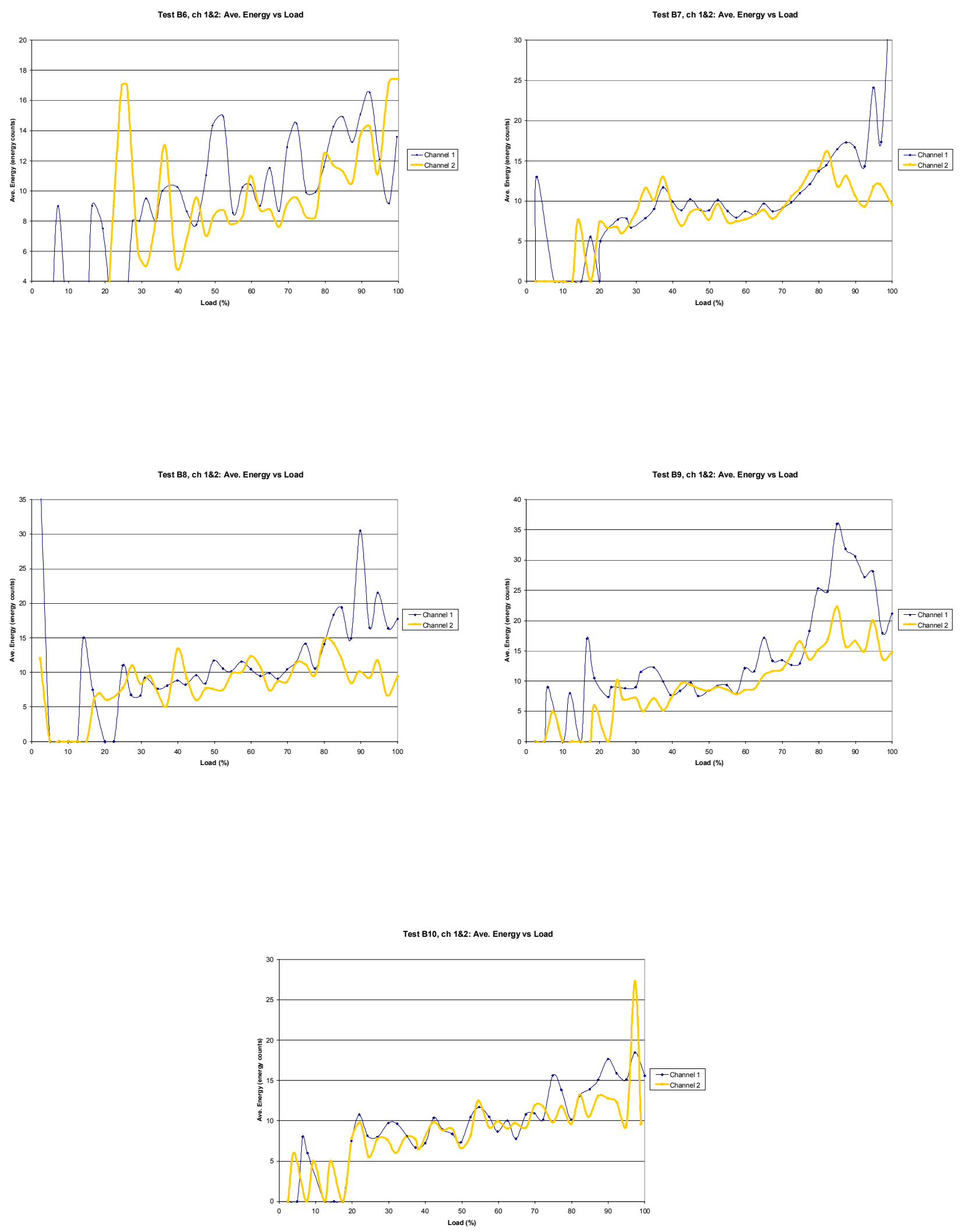

Figure 6.5 Average Energy vs. Load Parameter, Tests B6-B10 
Test C1, ch 182: Ave. Energy vs Load

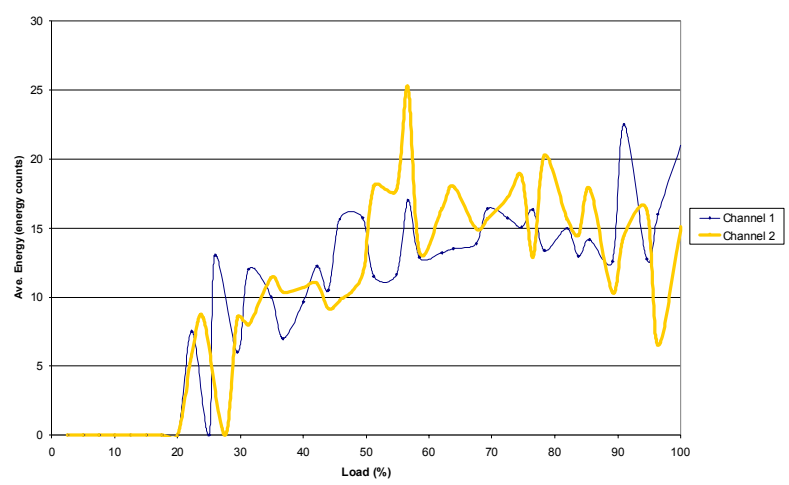

Test C3, ch 1\&2: Ave. Energy vs Load

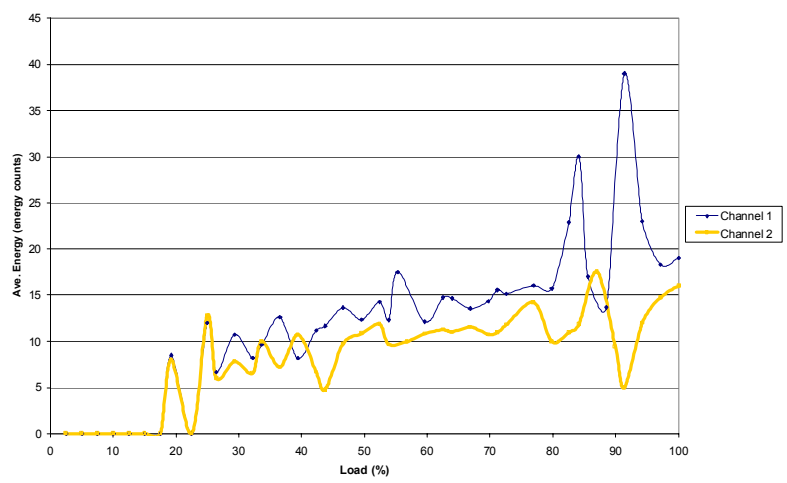

Test C2, ch 182: Ave, Energy vs Load

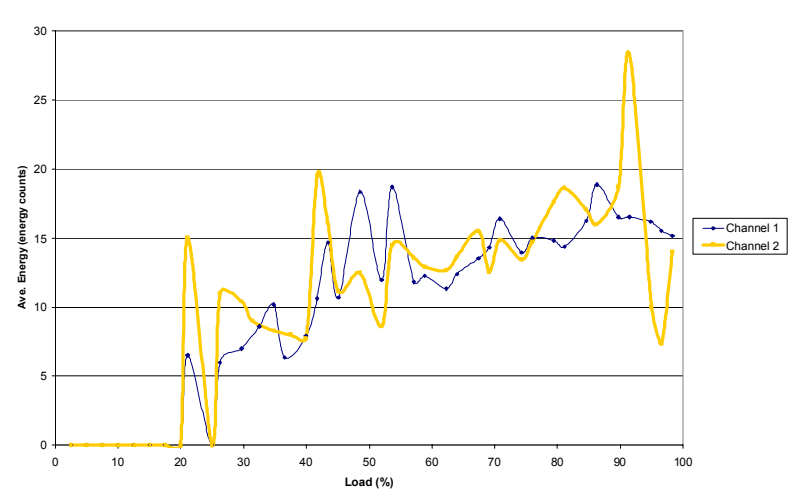

Test C4, ch 182: Ave. Energy vs Load

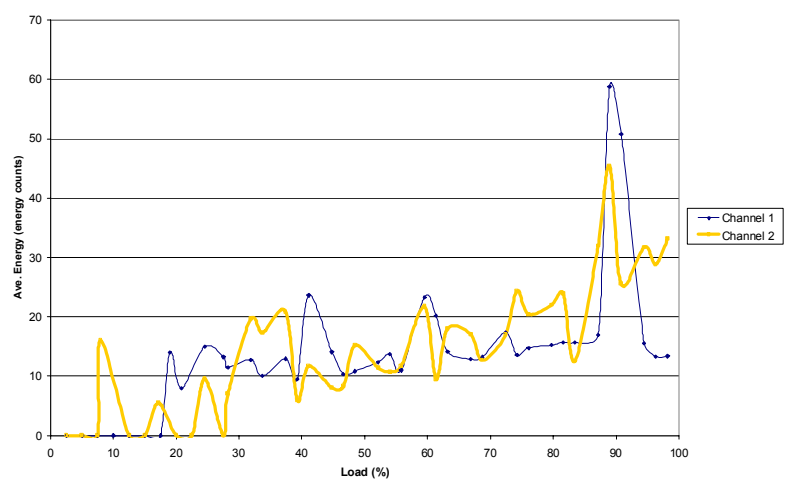

Test C5, ch 1\&2: Ave. Energy vs Load

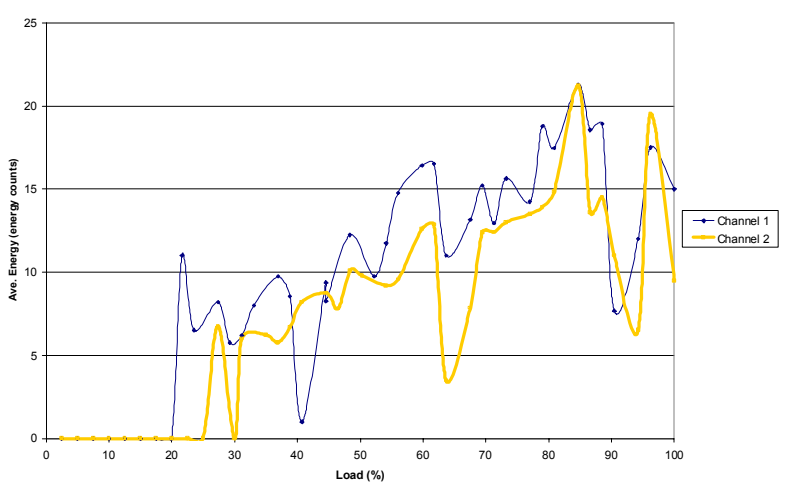

Figure 6.6 Average Energy vs. Load Parameter, Tests C1-C5 
Test B1, ch 1\&2: Ave. Duration vs Load

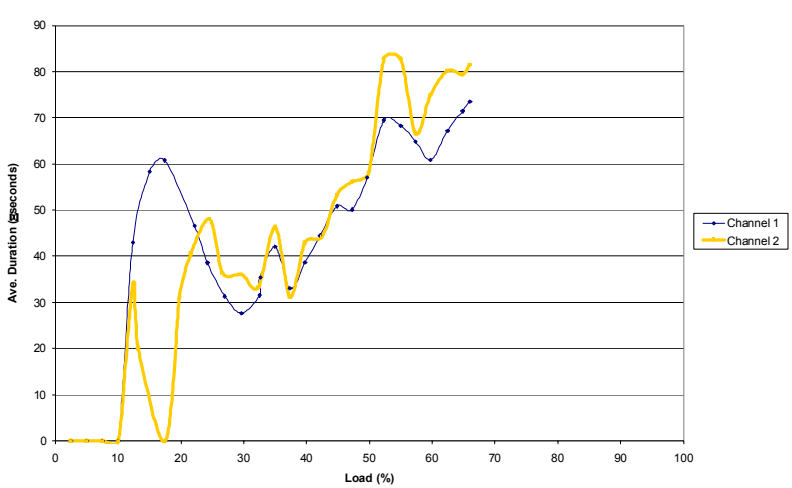

Test B3, ch 1\&2: Ave. Duration vs Load

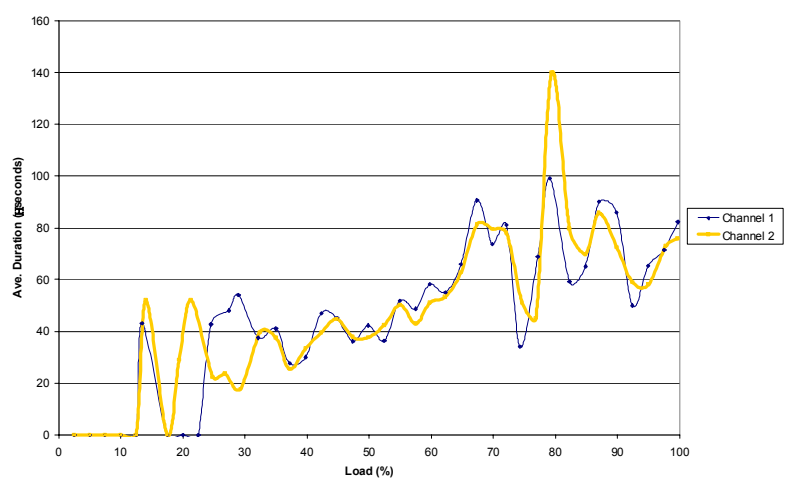

Test B2, ch 182: Ave. Duration vs Load

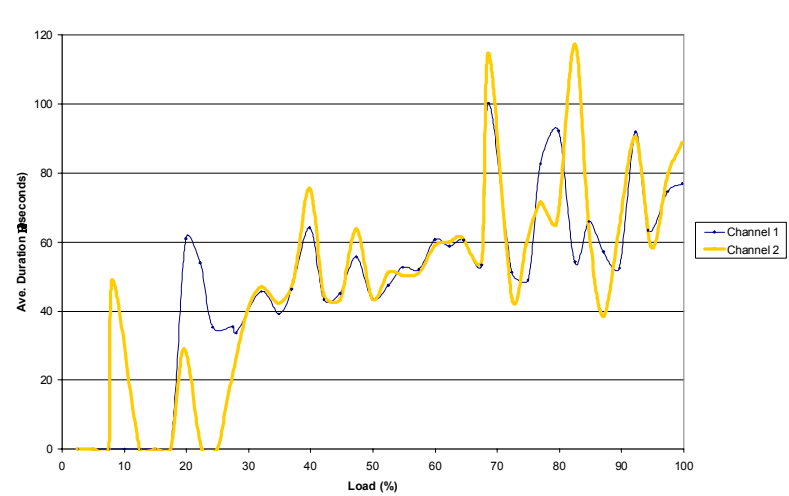

Test B4, ch 182: Ave. Duration vs Load

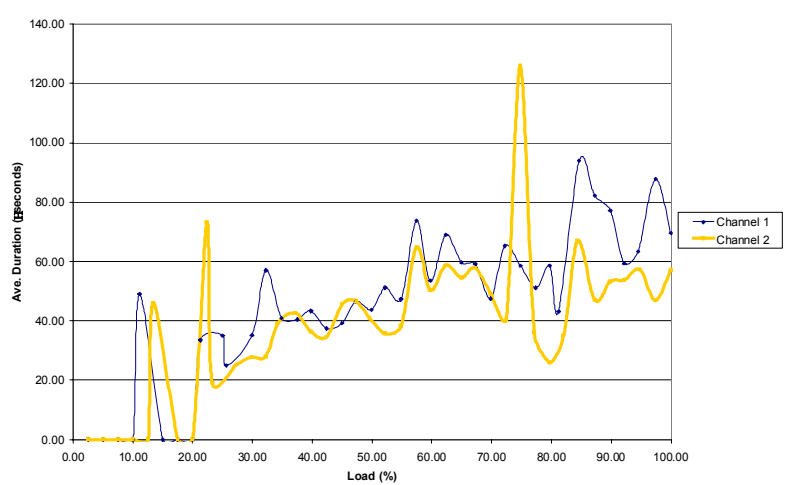

Test B5, ch 1\&2: Ave. Duration vs Load

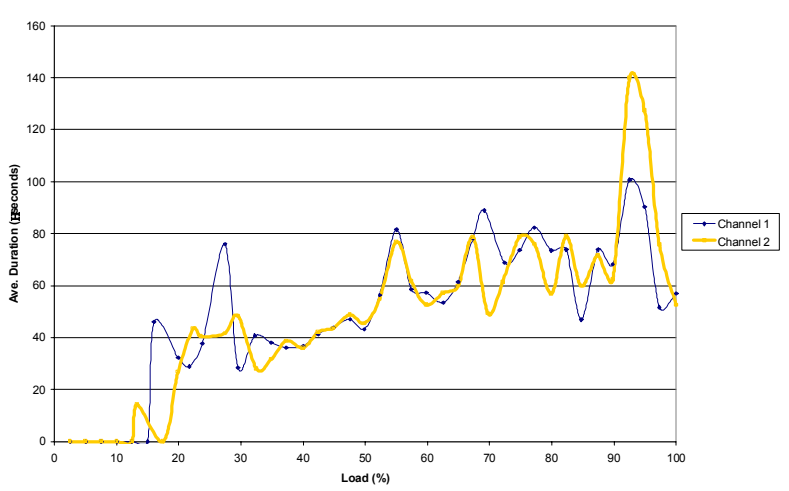

Figure 6.7 Average Duration vs. Load Parameter, Tests B1-B5 
Test B6, ch 1\&2: Ave. Duration vs Load

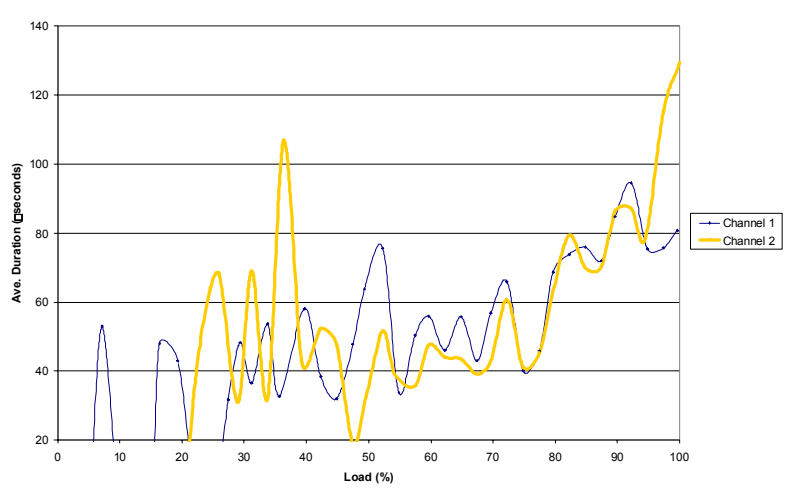

Test B8, ch 182: Ave. Duration vs Load

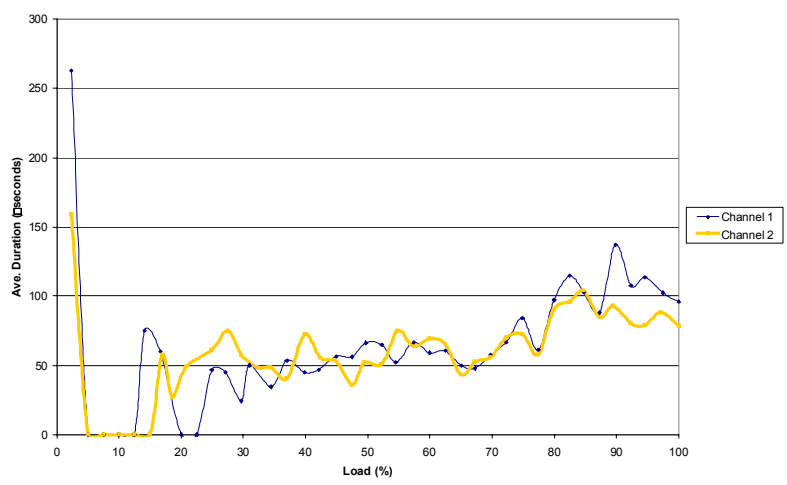

Test B7, ch 182: Ave. Duration vs Load

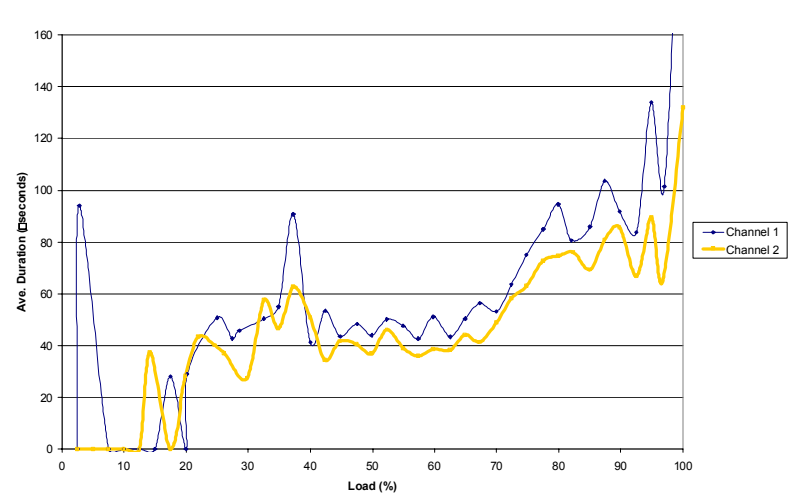

Test B9, ch 182: Ave. Duration vs Load

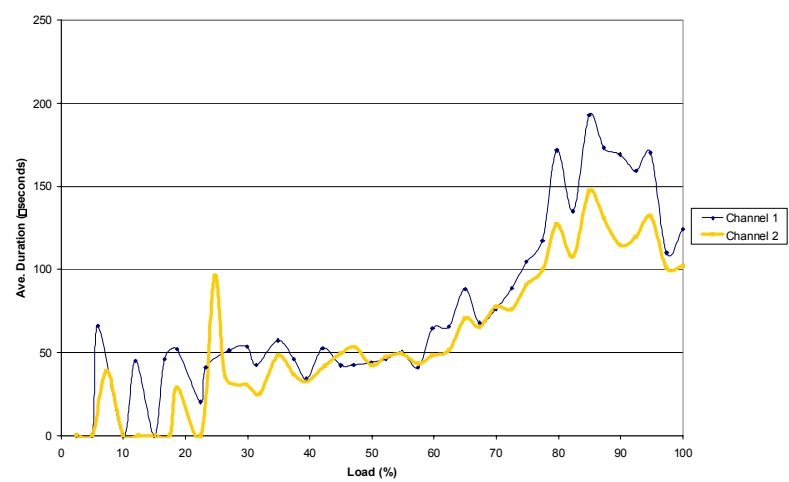

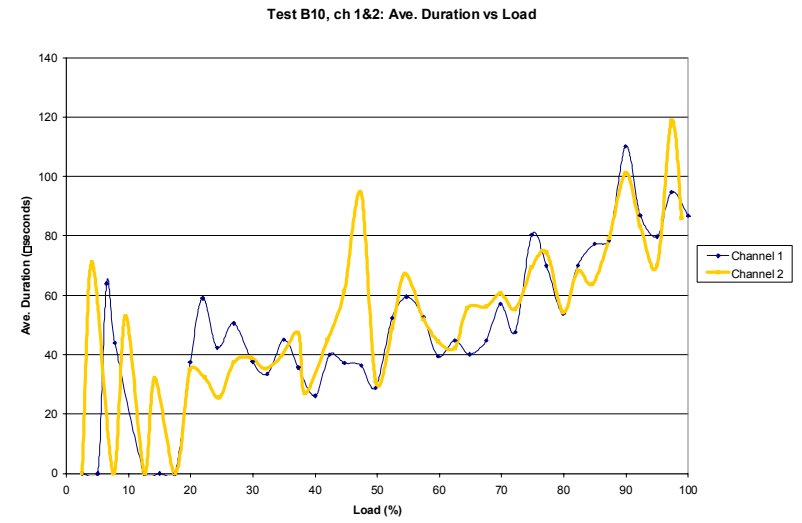

Figure 6.8 Average Duration vs. Load Parameter, Tests B6-B10 
Test C1, ch 1\&2: Ave. Duration vs Load

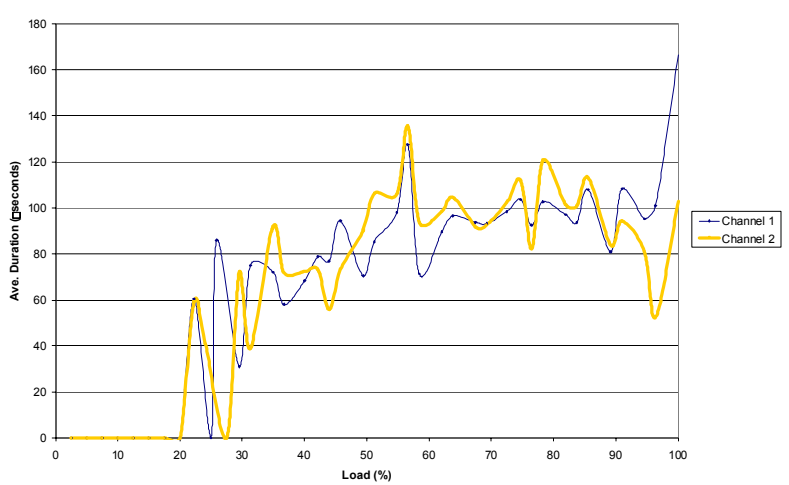

Test C3, ch 182: Ave. Duration vs Load

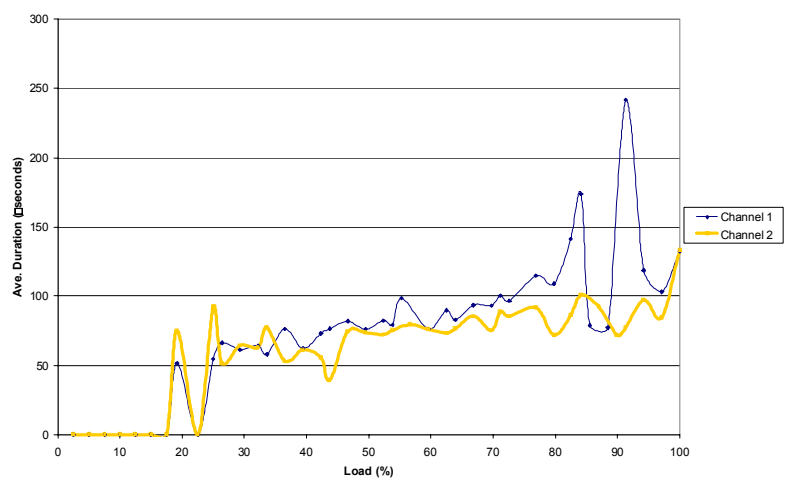

Test C2, ch 182: Ave. Duration vs Load

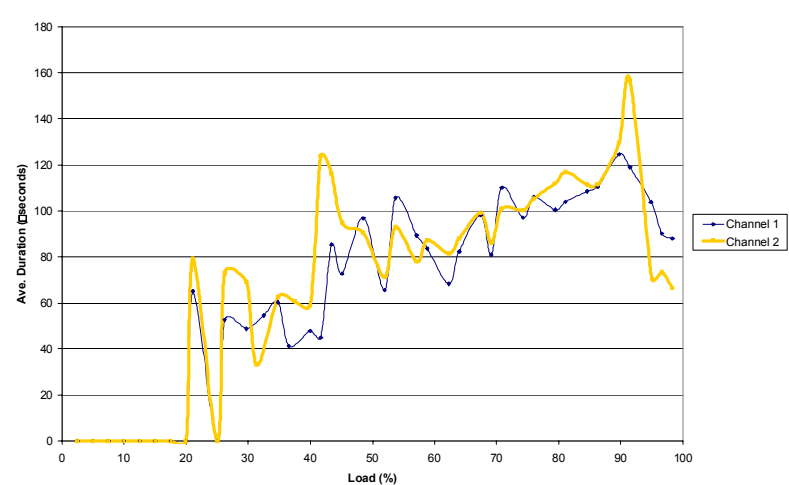

Test C4, ch 182: Ave. Duration vs Load

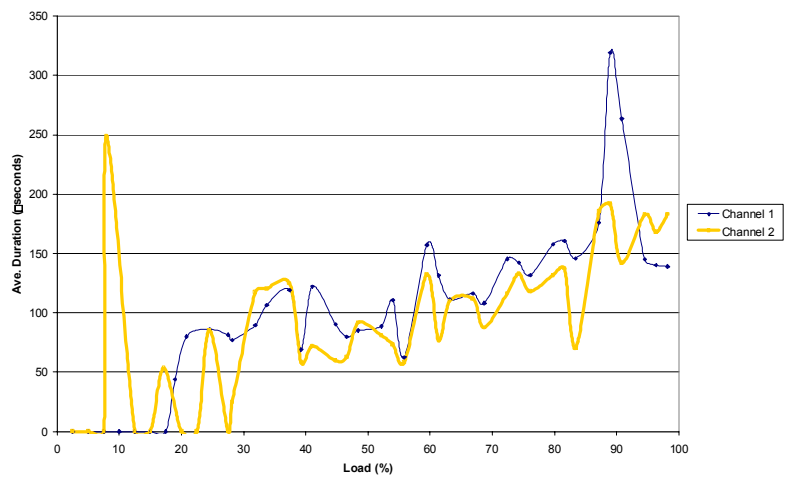

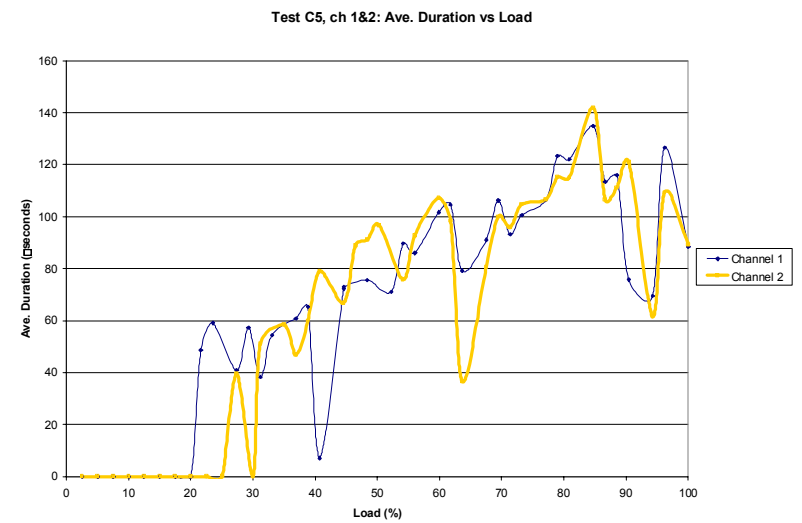

Figure 6.9 Average Duration vs. Load Parameter, Tests C1-C5 
Test B1, ch 182: Ave. Amplitude vs Load

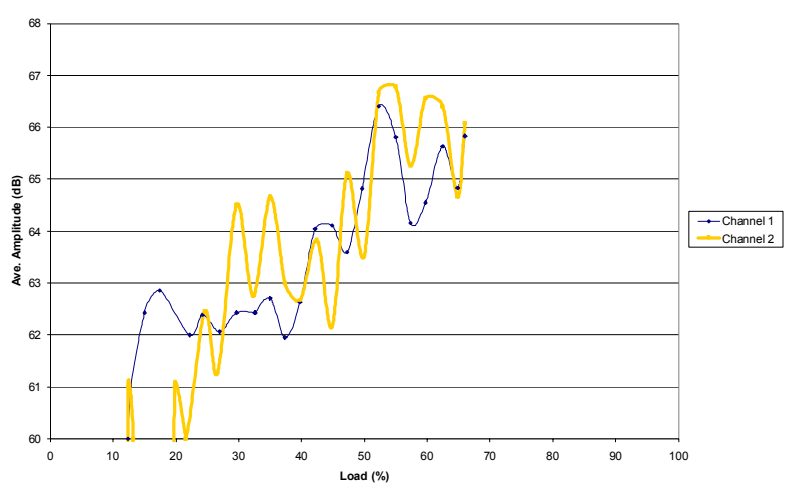

Test B3, ch 182: Ave. Amplitude vs Load

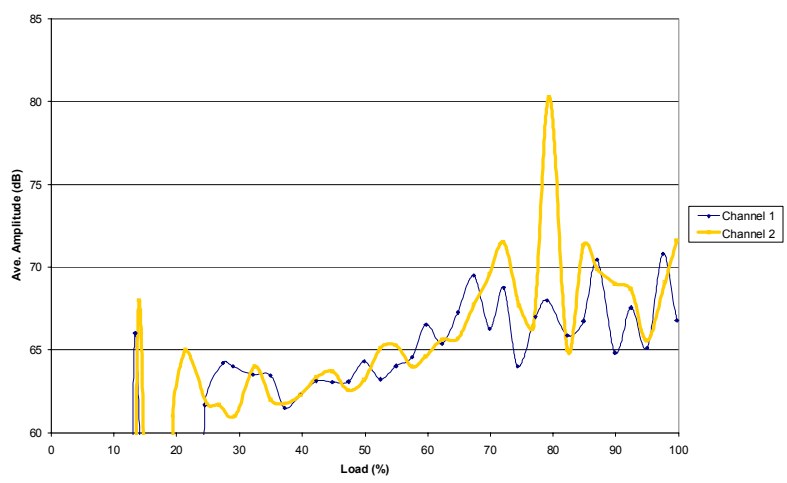

Test B2, ch 1\&2: Ave. Amplitude vs Load

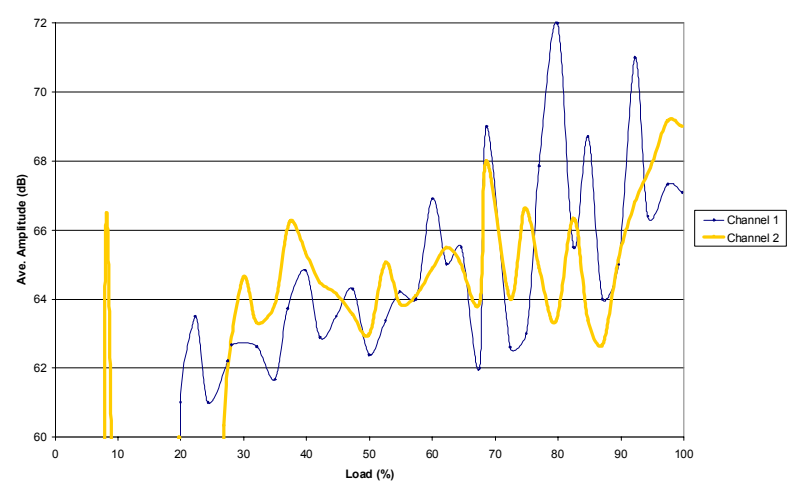

Test b4, ch 1\&2: Ave. Amplitude vs Load

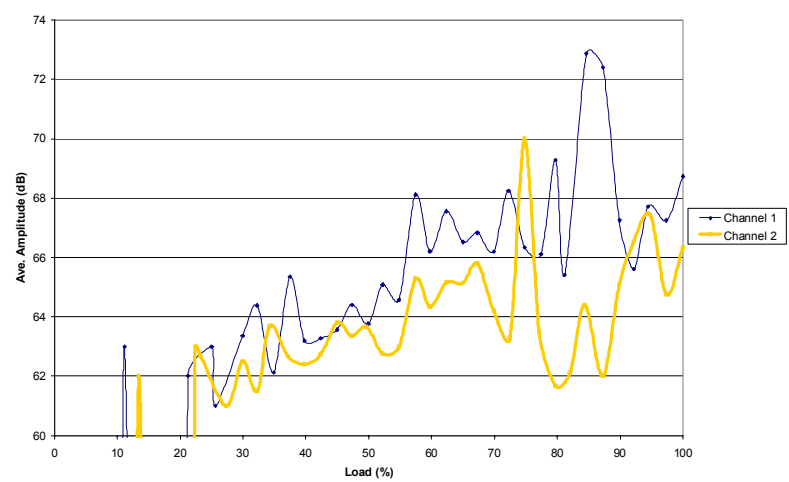

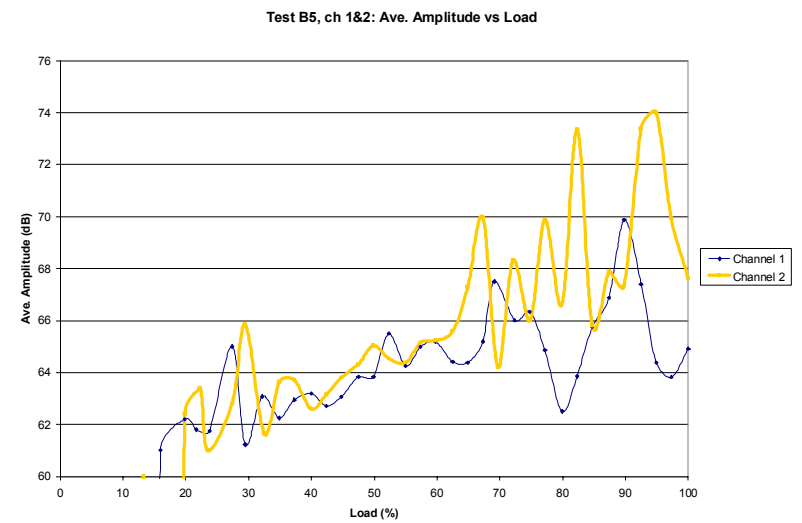

Figure 6.10 Average Amplitude vs. Load Parameter, Tests B1-B5 

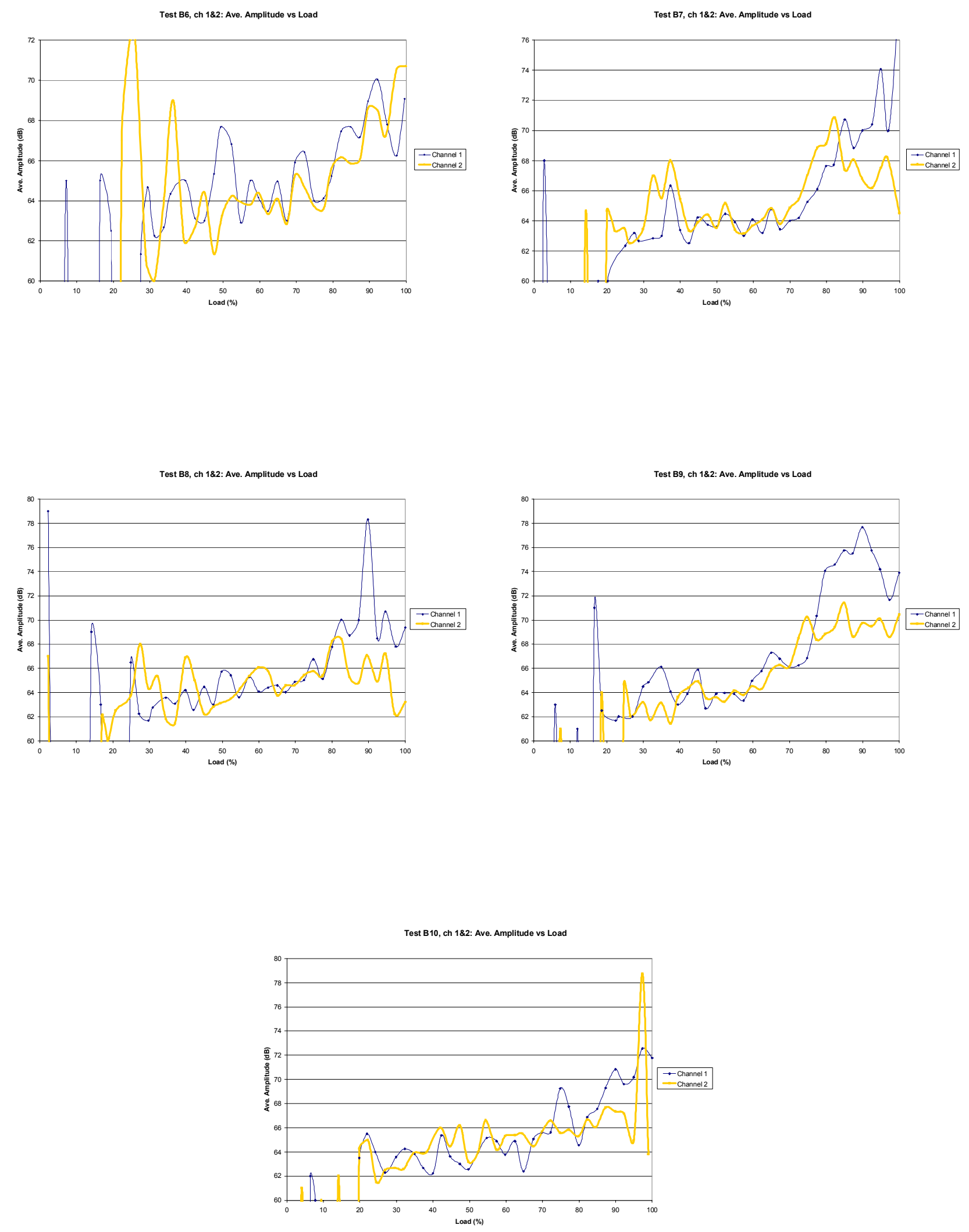

Figure 6.11 Average Amplitude vs. Load Parameter, Tests B6-B10 
Test C1, ch 1\&2: Ave. Amplitude vs Load

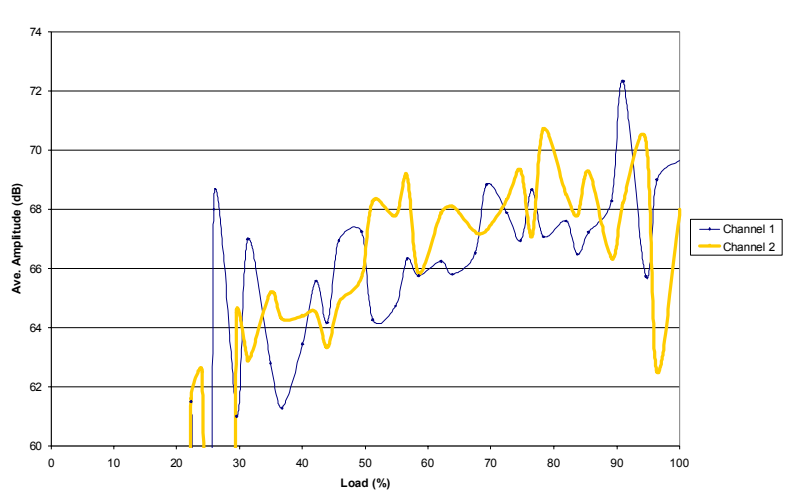

Test C3, ch 182: Ave. Amplitude vs Load

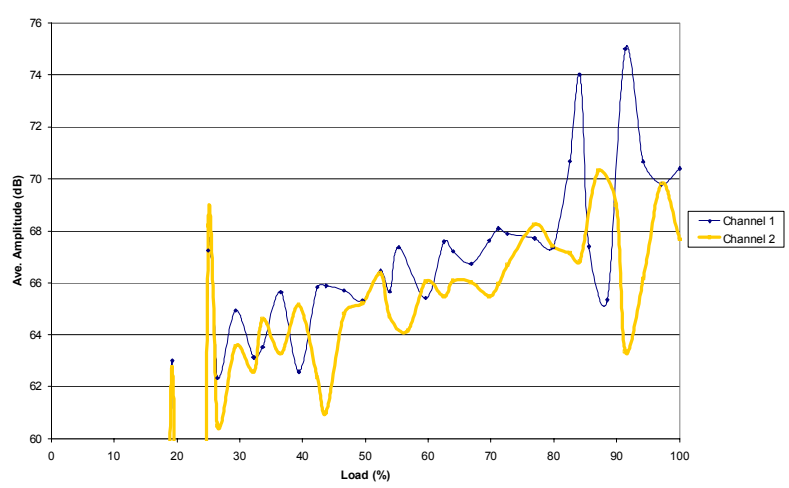

Test C2, ch 1\&2: Ave. Amplitude vs Load

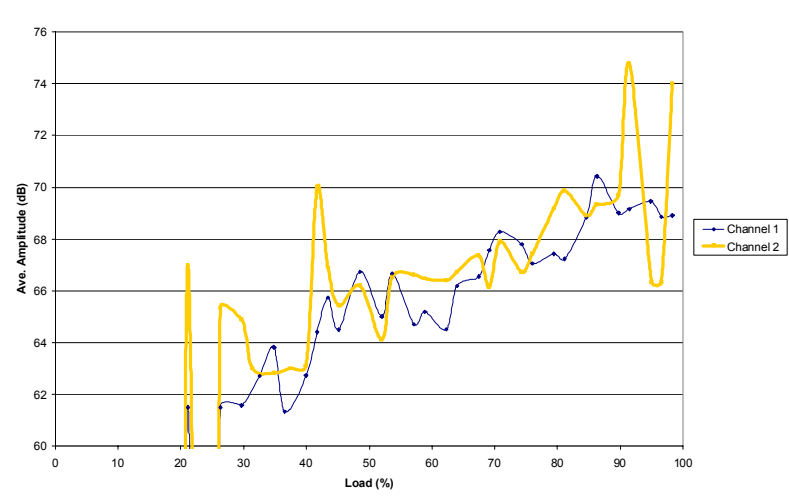

Test C4, ch 1\&2: Ave. Amplitude vs Load

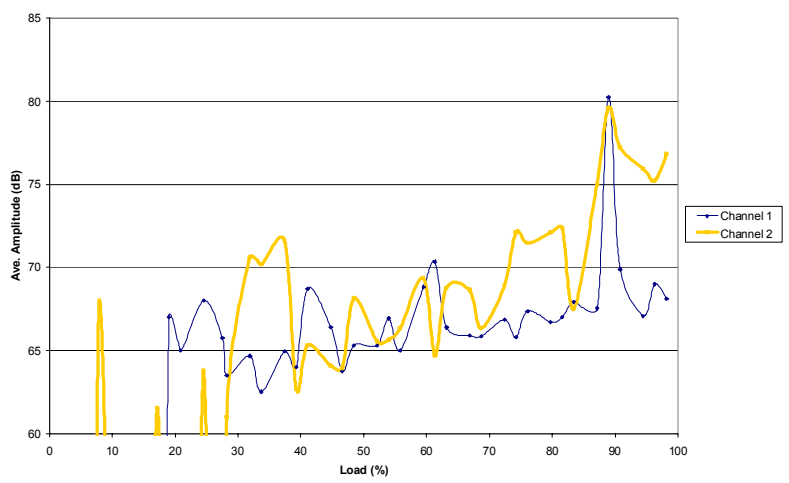

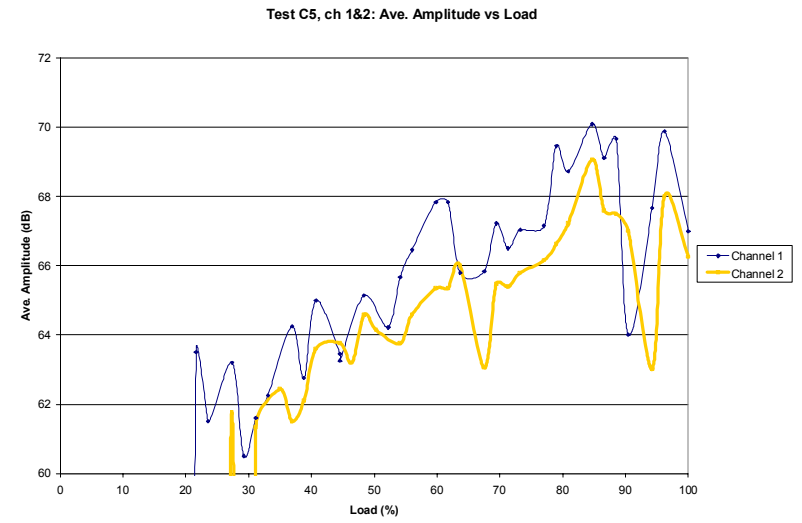

Figure 6.12 Average Amplitude vs. Load Parameter, Tests C1-C5 
Test B1, ch 182: Hits vs Load

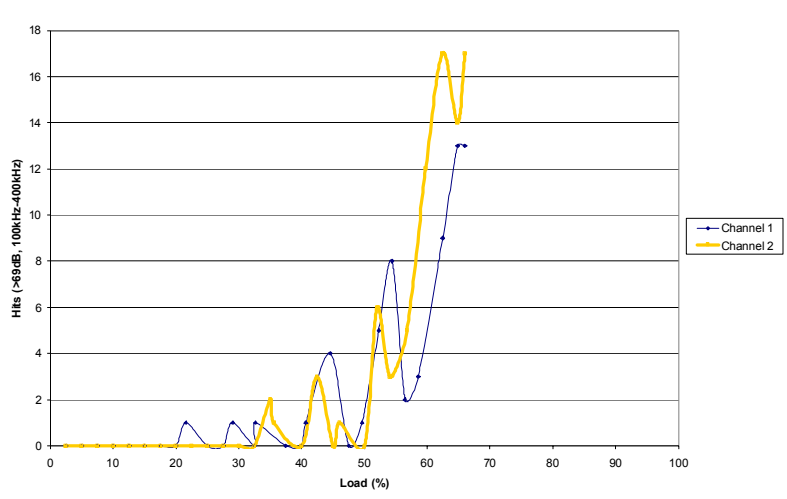

Test B3, ch 182: Hits vs Load

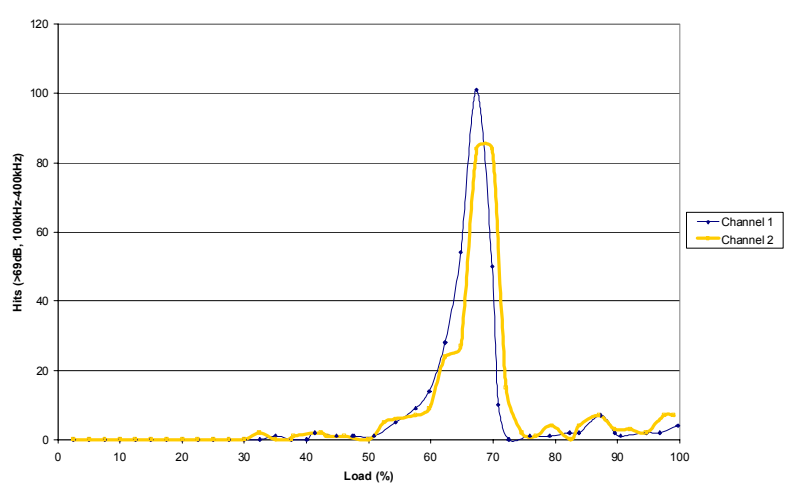

Test B2, ch 182: Hits vs Load

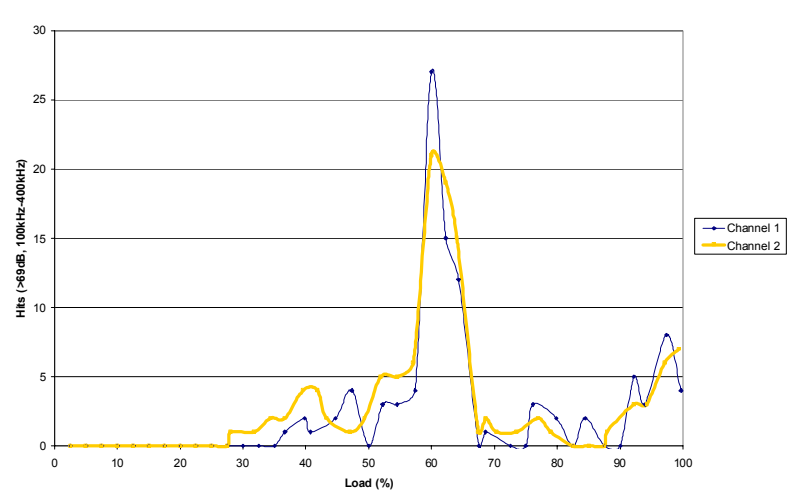

Test B4, ch 182: Hits vs Load

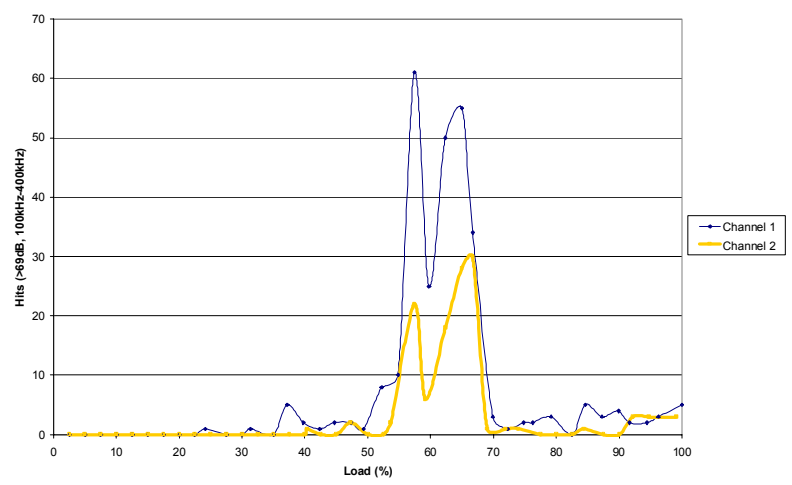

Test B5, ch 182: Hits vs Load

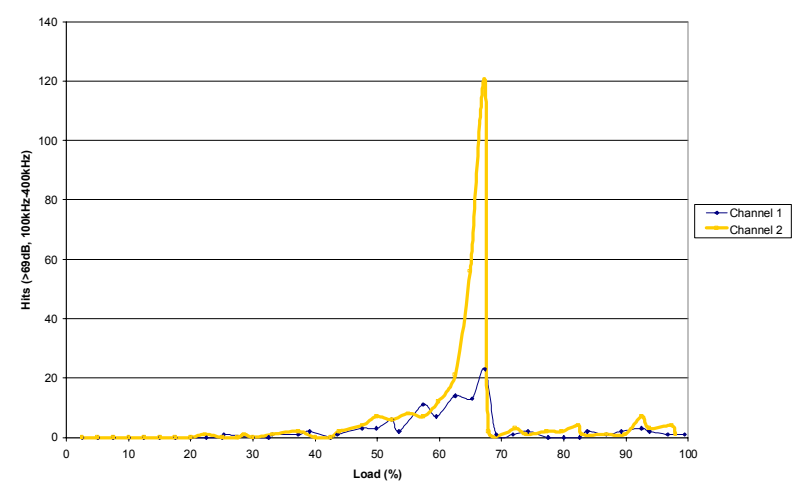

Figure 6.13 Frequency Hits vs. Load Parameter, Tests B1-B5 
Test B6, ch 1\&2: Hits vs Load

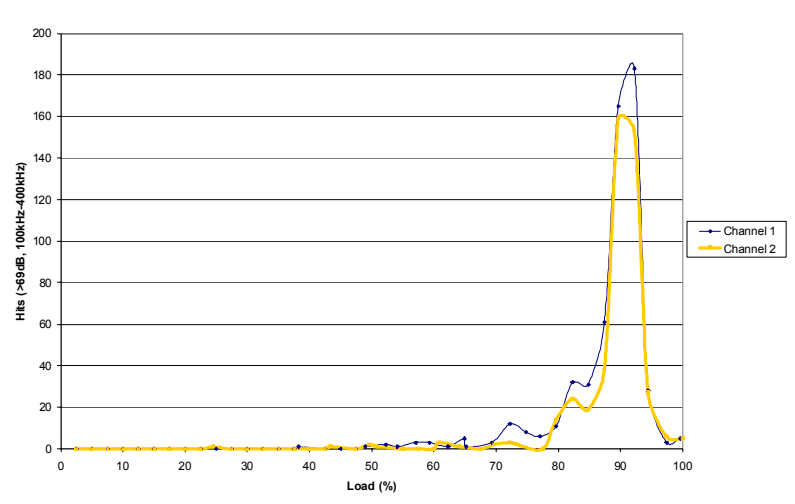

Test B8, ch 182: Hits vs Load

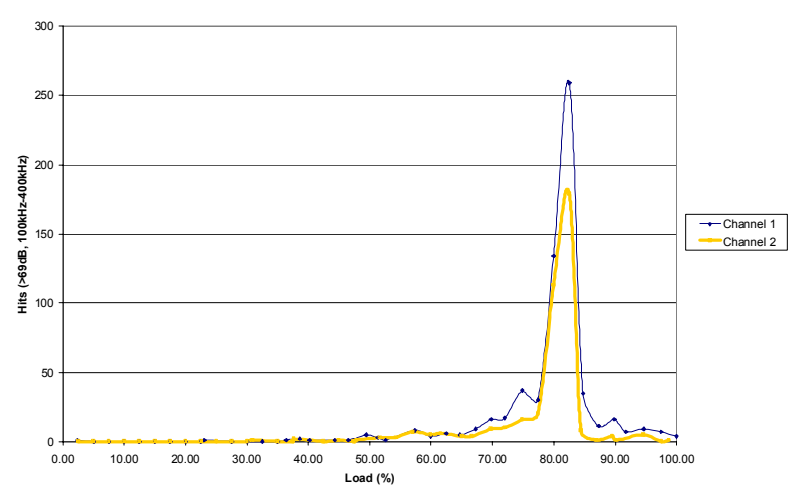

Test B7, ch 182: Hits vs Load

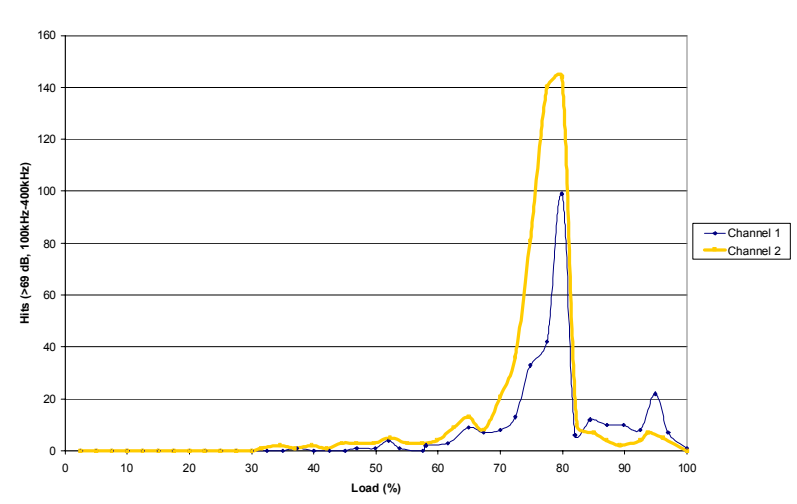

Test B9, ch 182: Hits vs Load

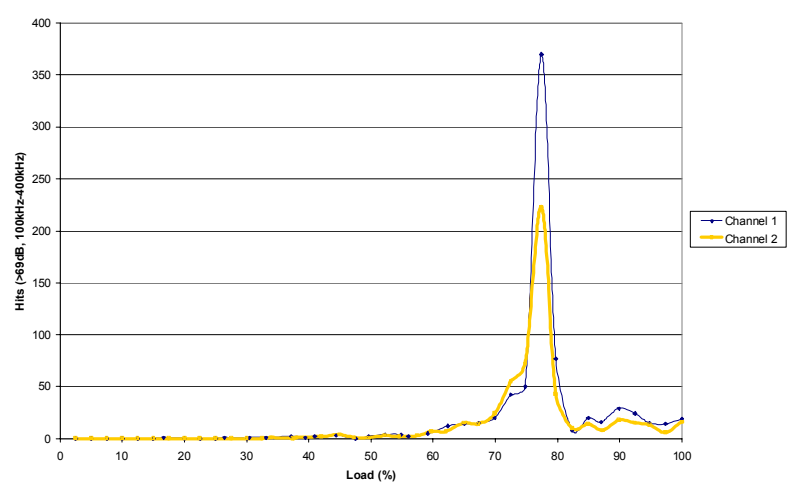

Test B10, ch 182: Hits vs Load

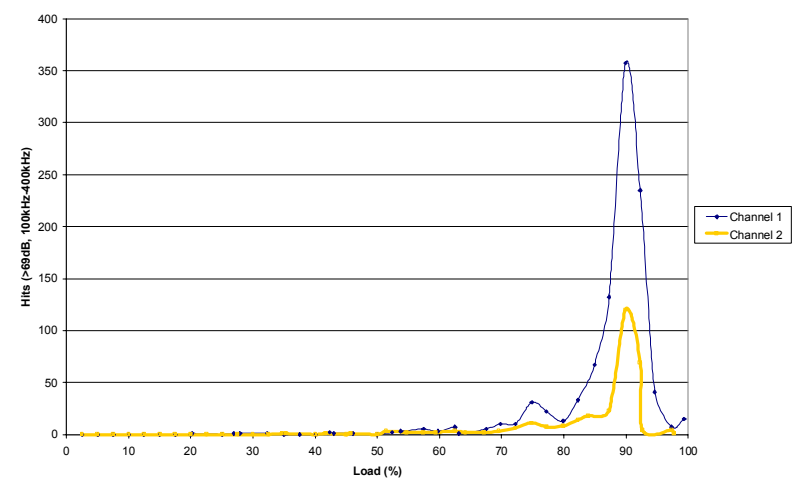

Figure 6.14 Frequency Hits vs. Load Parameter, Tests B6-B10 

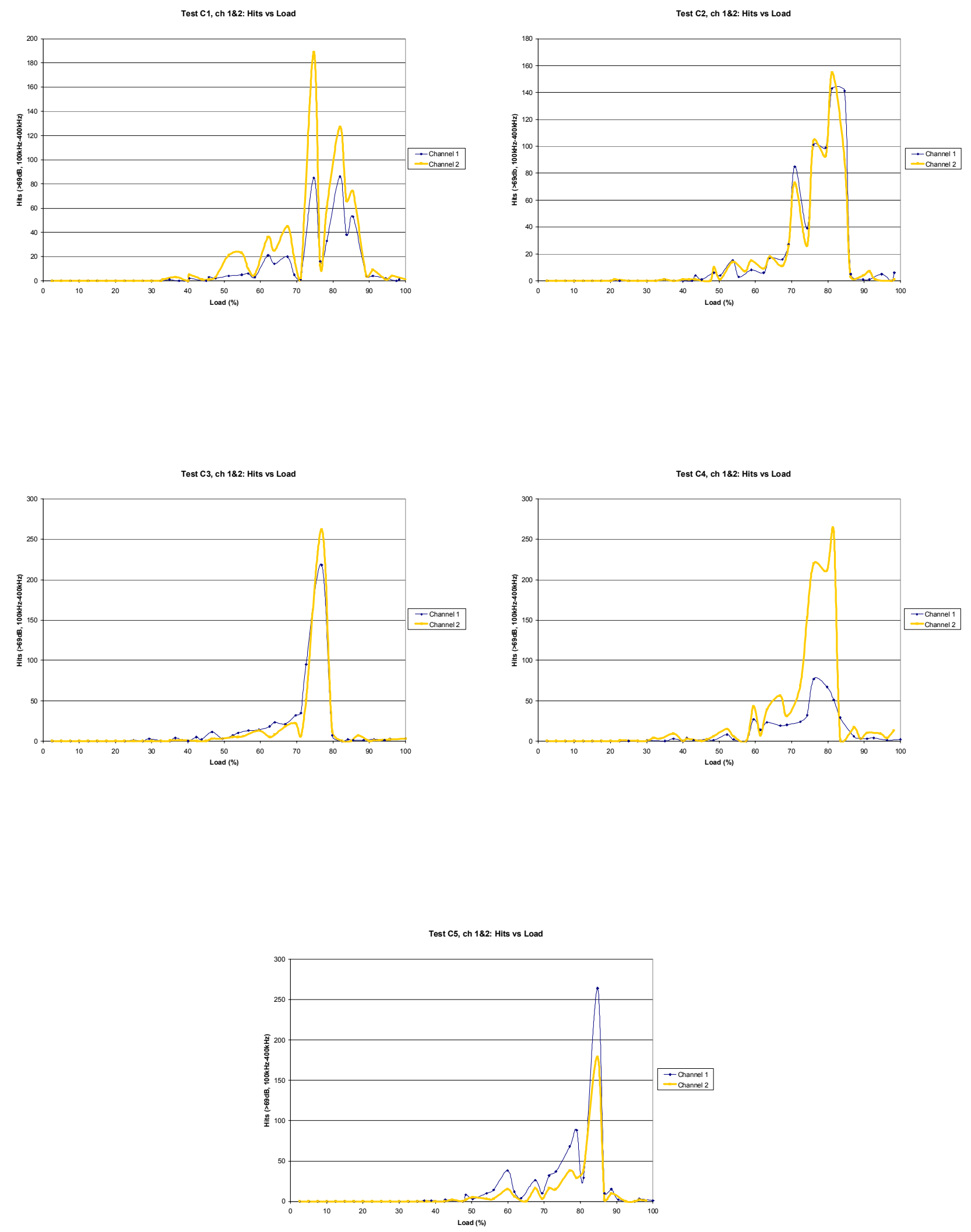

Figure 6.15 Frequency Hits vs. Load Parameter, Tests C1-C5 
Test B1, ch 1\&2: Sum of First Sensor Hit

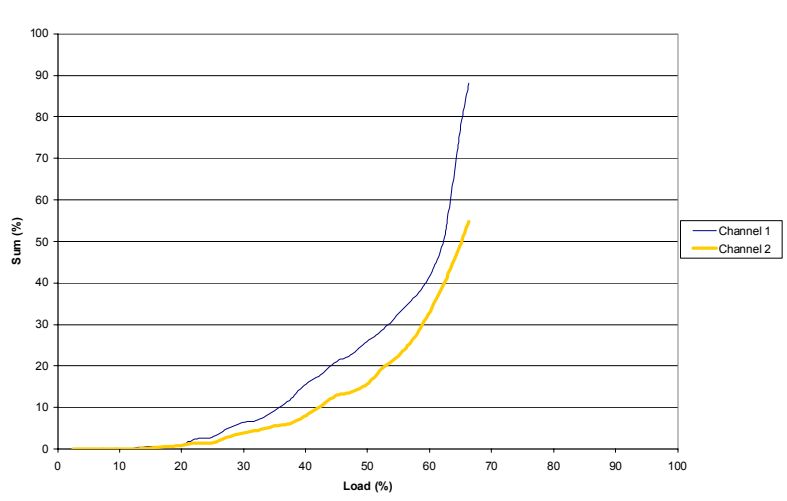

Test B3, ch 182: Sum of First Sensor Hit

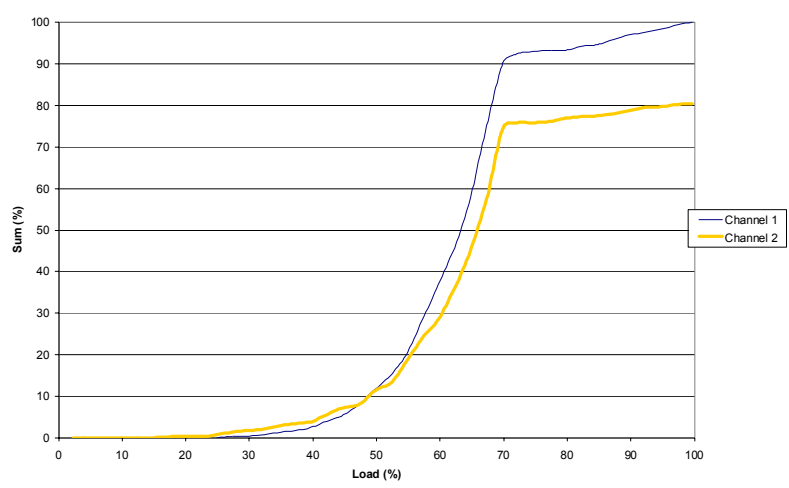

Test B2, ch 1\&2: Sum of First Sensor Hit

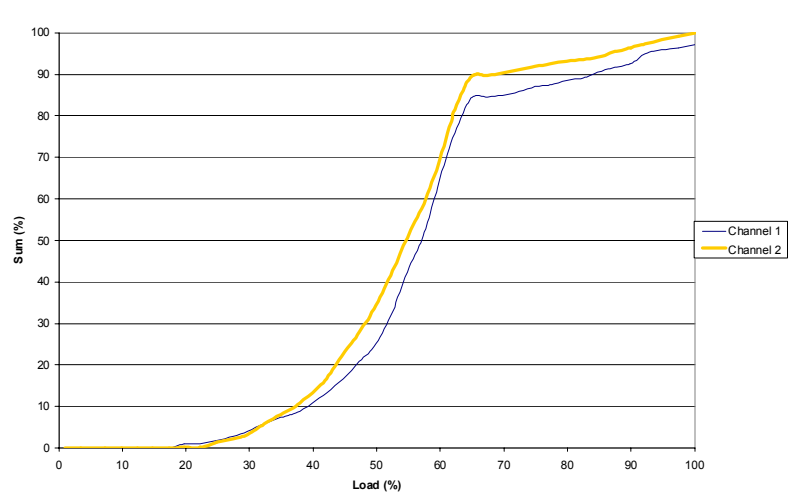

Test B4, ch 1\&2: Sum of First Sensor Hit

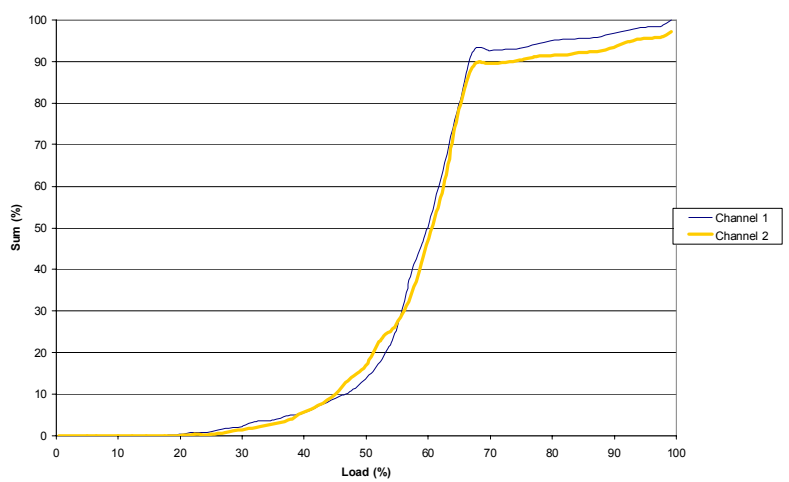

Test B5, ch 182: Sum of First Sensor Hit

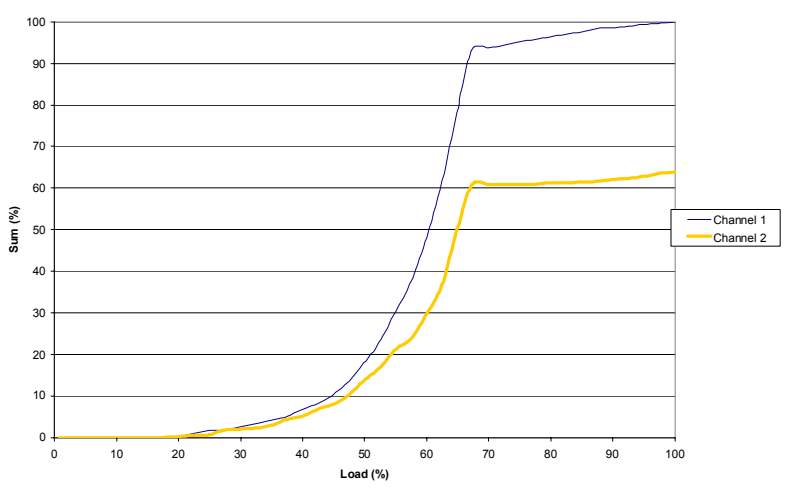

Figure 6.16 Sum of First Sensor Hit vs. Load, Tests B1-B5 

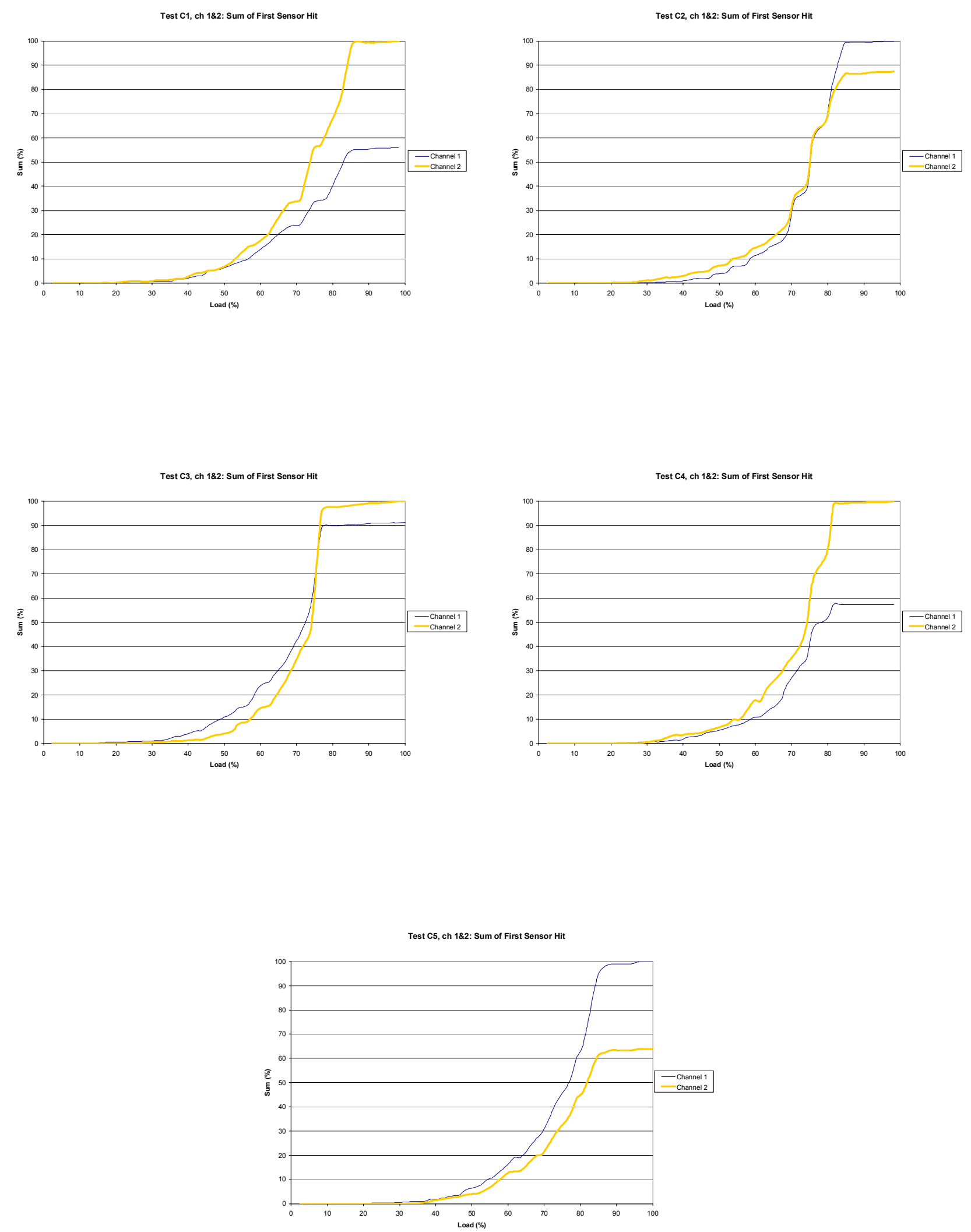

Figure 6.17 Sum of First Sensor Hit vs. Load, Tests C1-C5 


\section{Chapter 7}

\section{Conclusions, Applications, \& Recommendations}

\subsection{Conclusions}

In order to encourage the use of FRP composite members in the civil construction industry, an effective method of nondestructively monitoring the structure's integrity is needed. This study presents Acoustic Emission evaluation as a possible solution to this problem. The AE data processing methods along with the $\mathrm{NN}$ analysis procedures presented have resulted in an effective method for monitoring the integrity of FRP composite structures. This could be used in the future on actual in-situ structures. This could lead to a real-time health monitoring system for FRP structures that eliminates human error.

The following are among the specific conclusions attained from this research. Acoustic Emissions Study

1. Signals of high amplitude $(>69 \mathrm{~dB})$ falling in a certain frequency range $(100 \mathrm{kHz}-400$ $\mathrm{kHz}$ ) give warning that the FRP structure is nearing its failure load. The signals of lower amplitude are associated with any noise present.

2. Signals with higher amplitudes and higher durations also indicate that the structure is nearing its failure load. As the loading increases, the amplitude vs. duration graphs tend to display an explosion outward from the origin of the graph.

3. The summation energy vs. load graphs display a distinctive turning point followed by decreased slope. This decreased slope also warns of approaching failure loads.

4. Exactly locating the failure of FRP structures is very complicated due to their complex architecture. Absolute location is also hindered by the fact that many cracks occur near the end of the loading increasing material attenuation and decreasing the 
wavespeed through the specimen. However, the sensor that is hit the most often first by locatable signals gives a good indication of the area in which the structure will fail. Because of this, the damage zone method is a more effective method than absolute location.

5. Tension tests and bending tests with sensors mounted near the tension area result in AE parameter values that show similar trends. This implies a consistent link between loading quarter and $\mathrm{AE}$ parameters.

6. Similarly, specimens from the hand lay-up method and from commercial manufactures show the same trends. Specimens from different manufacturers and of different architecture also show these same AE trends. This points to the conclusion that the $\mathrm{AE}$ parameters are related to the loading quarter. This means that these relations are characteristics of FRP materials in general and are independent of the manufacturer, type of manufacturing process, or specimen architecture used in this study.

7. The AE data processing methods found have proven to be an effective method for analyzing FRP structures. This includes the extraction of useful data from immense sets of data, the filtration of true signal data, and the condensing of this data for use with NN analysis. These methods are described in detail in Chapter 5.

Neural Network Analysis

1. Neural network (NN) analysis is an effective method for predicting structural status using AE parameters.

2. An effective $\mathrm{NN}$ analysis method was found using the average $\mathrm{AE}$ parameter over a certain loading period. In this case, averages for AE parameters of counts, energy, 
duration, amplitude, and frequency hits using normalized $\mathrm{AE}$ data to provide equal weighting for periods of $2.5 \%$ of the failure loading were found useful.

3. Prediction of loading becomes more accurate as the loading reaches more critical stages (failure loading). Damage zone prediction shows great promise in being able to locate areas of increased damage allowing for repairs to be made before the specimen completely fails.

4. Specimens from different manufacturers and of different internal architecture can be combined and while not as entirely effective as separated groups, still render effective prediction capabilities.

\subsection{Applications}

The experimental results and conclusions made from them can be applied in the following future applications.

1. The connections made between AE parameters and a structure's status can be applied to future structures on larger scales. Eventually this can lead to evaluation of the health of actual bridge structures.

2. The method of AE data processing presented in this research can be used to extract useful data from data sets of immense size. This can be done in real-time with limited computer programming.

3. Application of the NN methods found to other FRP structures (laboratory and field), could provide effective prediction results for the health of that structure. 


\subsection{Recommendations}

Based on the conclusions and applications stated above, the following recommendations are made.

1. Laboratory tests on larger scale specimens are needed in order to verify that the findings from the tension and bending specimens translate to larger scale specimens.

2. Additional tests on specimens from the same manufacturer or on specimens of the same architecture will most likely result in more accurate NN predictions. Additional data will generally make $\mathrm{NN}$ predictions more accurate.

3. The method of slope difference may be effectively used for square layouts as well as linear layouts. Figure 7.1 shows a schematic of a possible configuration for a four sensor square layout. On each of the four corners, is a sensor. In this case, the $\mathrm{x}$ coordinate is found from the average of $2-1$ and $4-3$. For the y coordinate, the average is taken of 1-3 and 2-4. If the $\mathrm{x}, \mathrm{y}$ coordinate for an event is found to approximately 0, 0 it implies the signal is near the middle. Likewise an event that has coordinates that are both positive indicates an event near to sensor 2. The higher the magnitude, the closer to sensor 2 it is located. This is repeated for the other sensors and is represented in the figure. This method is just a theoretical possibility and has not been tested.

4. When monitoring a structure in a real-time situation, normalization could present quite a problem. The maximum value for any parameter is not known until after it is achieved, at which point it may be too late to save that structure. In order to use the same method of normalization, the only feasible solution is to set a range for each parameter. This range would be established by averaging the minimum and 
maximum values for many tests. The range would then be used to normalize realtime data. Another possible solution is a running maximum value. In this case, the maximum value would continue to increase until the point it reaches its maximum. At that time, the maximum would stay set. In either case, the minimum value is not a problem due to the fact that it is established in the beginning.

5. The method of AE parameters connected to structural status along with NN predictions could be a great aid in the evaluation of FRP bridge deck structures. It is recommended that the same method be tested on larger scale objects with an eye on the development of a real-time health monitoring system for these structures. Such a system could be developed as a remote system. A system using NN or a like method to make predictions could help eliminate the possibility of user error. 


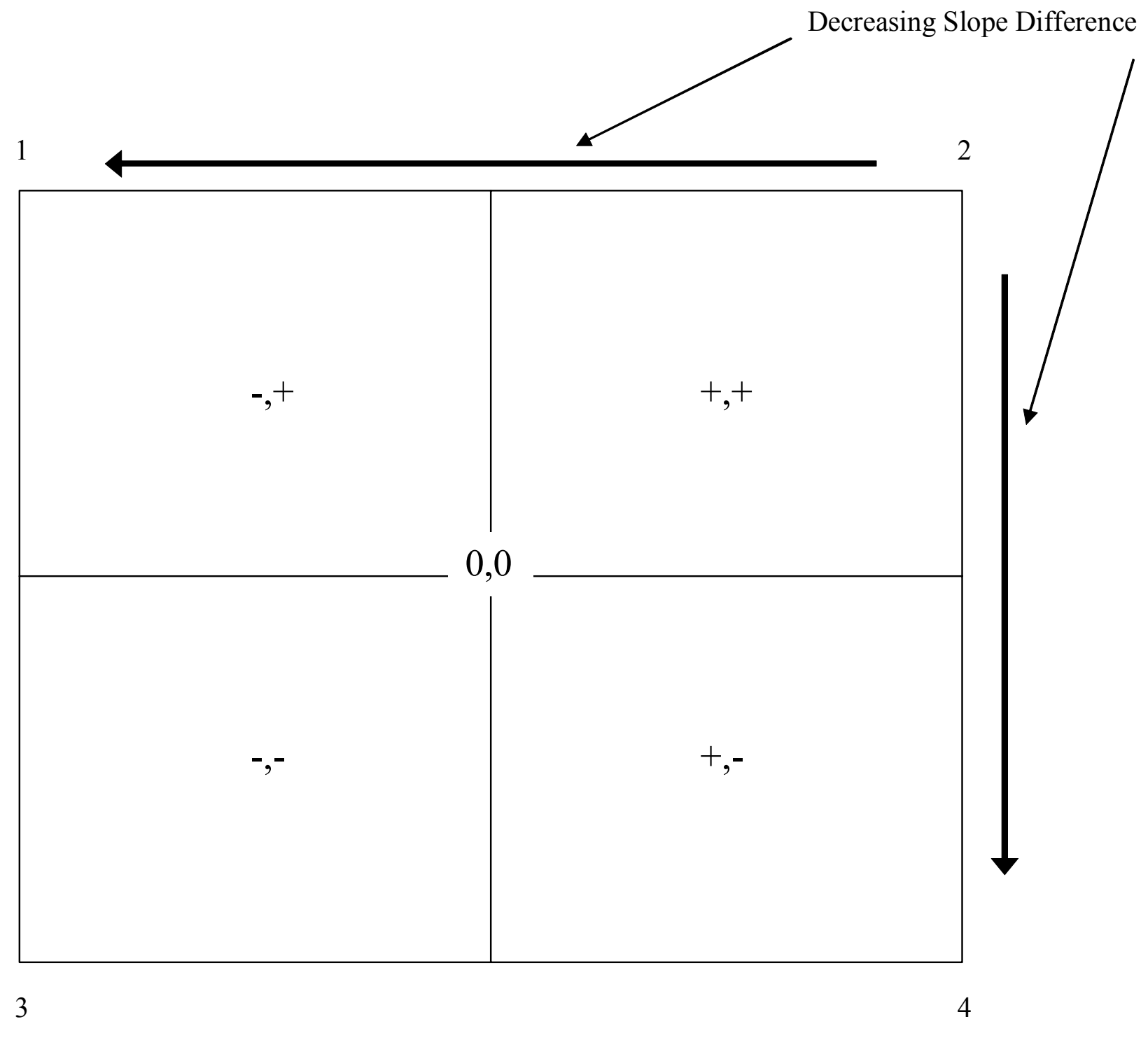

Figure 7.1 Possible Technique for Damage Zone Predictor Using Sensors in a Rectangular/Square Layout 


\section{References}

1. ASTM Standards.

2. Chen, C. L., "Training the Multi-layer Neural Nets by Setting the Initial Weights," Ph.D. Dissertation, West Virginia University, Morgantown, 1992.

3. Chen, H. L., and Chen, C. L., " Applying Neural Network to Acoustic Emission Signal Processing", Fourth International Symposium on Acoustic Emission From Composite Materials, The American Society for Nondestructive Testing, Seattle, Washington, pp. 273-281, July, 1992.

4. Chen, H. L., Cheng, C. T., Chen, S. E., "Determination of Fracture Parameters of Mortar and Concrete Beams by Using Acoustic Emission", Materials Evaluation, pp. 888-894, July, 1992.

5. Chen, H. L., He, Yidong, and Superfesky, Michael, "Acoustic Surface Waveguides for Acoustic Emission Monitoring of Fiber-Reinforced Plastic Structures', Materials Evaluation, Vol. 52, No. 9, pp. 1112-1116, September, 1994.

6. Chen, H. L., Sami, Z., and GangaRao, H. V., " Identifying Damages in Stressed Aramid FRP Bars Using Acoustic Emission", Dynamic Characterization of Advanced Materials, ASME, NCA-Vol. 16/ AMD-Vol. 172, pp. 171-178, 1993.

7. Chen, H. L., and Wissawapaisal, Komwut, "Study of Acoustic Surface Waveguides on Reinforced Concrete Slabs" Journal of Nondestructive Evaluation, Vol. 19, No. 4, 2000.

8. Chen, H. L., Zhou, H. W., and GangaRao, H. V., "Characterization of West Virginia Hardwood Using Acoustic Emission and Vibration Method", Review of Progress in Quantitative Nondestructive Evaluation, Vol. 11, Thompson, D. O., and Chimenti, D. E. ed., Plenum Press, New York, 1992.

9. Choi, J. H., and Chen, H. L., "Design Considerations of GFRP-Reinforced CRCP," accepted for ACI Special Publication for Sessions on "Field Applications of FRP Reinforcement: Case Studies" for the Boston Convention, Sept. 27-Oct. 1, 2003.

10. Choi, Jeong-Hoon, "The Fracture Analysis and Remaining Life Estimation of the AVLB Sub-Components", Master's Thesis, West Virginia University, Morgantown, November 2000 .

11. Eberhart, Russ, Simpson, Pat, and Dobbins, Roy, Computational Intelligence PC Tools, An Indispensible Resource for the Latest in: Fuzzy Logic, Neural Networks, Evolutionary Computing, AP Professional, Orlando, Florida, 1996. 
12. Fiber Reinforced Polymer Composite Bridges of West Virginia, FHWA Pub. No. FHWAERC-2-002, Compiled by Constructed Facilities Center, West Virginia University, Morgantown, West Virginia, 2001.

13. "FRP Materials, Manufacturing Methods and Markets", Composites Technology YellowPages, pg. 6, 1999

14. Fultineer, R. D., "Study of Fatigue Cracks in Steel Bridge Components Using Acoustic Emission”, Master's Thesis, West Virginia University, Morgantown, 1997.

15. Grabec, Igor, Sachse, Wolfgang, and Govekar, Edvard. "Solving AE Problems by a Neural Network", Acoustic Emission: Current Practice and Future Directions, ASTM STP 1077, Sachse/Roget/Yamaguchi ed., pp. 165-182, 1991.

16. Graff, Karl F., Wave Motion in Elastic Solids, Dover Publications, 1991.

17. Harrold, R. T., and Sanjana, Z. N., "Acoustic Waveguide Monitoring of the Cure and Structural Integrity of Composite Materials", Polymer Engineering and Science, Vol. 26, No. 5, mid-March, 1986.

18. Hawkins, Neil M., McCabe, W. Martin, and Nobuta, Yoshinobu, "Use of Acoustic Emission to Detect Debonding of Reinforcing Bars in Concrete", Progress in Acoustic Emission IV, The Japanese Society for NDI, 1988.

19. Higo, Yakichi, and Hidehiro, Inaba, "The General Problems of AE Sensors", Acoustic Emission: Current Practice and Future Directions, ASTM STP 1077, Sachse/Roget/Yamaguchi ed., pp. 7-24, 1991.

20. McIntire, Paul, Nondestructive Testing Handbook, $2^{\text {nd }}$ ed., Vol. 5 Acoustic Emission Testing, ASNT, 1987.

21. Mitchell, Tom M., Machine Learning, WCB/McGraw-Hill, Portland, Oregon, 1997.

22. O’Connor, Jerome S., "New York's Experience with FRP Bridge Decks”, Polymer Composites II: Applications of Composites in Infrastructure Renewal and Economic Development, Creese, Robert C., and GangaRao, Hota ed., Organized by Constructed Facilities Center, West Virginia University, Morgantown, West Virginia, 2001.

23. PCI, PCI Design Handbook: Precast and Prestressed Concrete, Fourth Edition, Chicago Illinois, 1992.

24. Pollock, A. A., "Practical Guide to Acoustic Emission Testing", LOCAN AT User's Manual, REV. 1.0, 1988.

25. Reeve, Scott R., "FRP Composite Bridge Decks: Barriers to Market Development",Polymer Composites II: Applications of Composites in Infrastructure 
Renewal and Economic Development, Creese, Robert C., and GangaRao, Hota ed., Organized by Constructed Facilities Center, West Virginia University, Morgantown, West Virginia, 2001.

26. Robert, Jeffrey L., "Replacement of the Bridge on MD 24 over Deer Creek in Harford County, Maryland, Utilizing a Fiber-Reinforced Polymer (FRP) Deck", Polymer Composites II: Applications of Composites in Infrastructure Renewal and Economic Development, Creese, Robert C., and GangaRao, Hota ed., Organized by Constructed Facilities Center, West Virginia University, Morgantown, West Virginia, 2001.

27. Soneji, J., Hu, C., Chaudhri, M., Faqiri, A. Gillespie, J. W., Jr., Eckel, D. A., II, Mertz, D. R., and Chajes, M.J., "Use of Glass-Fiber-Reinforced Composite Panels to Replace the Superstructure for Bridge 351 on N387A over Muddy Run", Polymer Composites II: Applications of Composites in Infrastructure Renewal and Economic Development, Creese, Robert C., and GangaRao, Hota ed., Organized by Constructed Facilities Center, West Virginia University, Morgantown, West Virginia, 2001.

28. Tong, L., Bannister, M., Mouritz, A., and Oakeley, A., 3D Fibre Reinforced Polymer Composites, $1^{\text {st }}$ ed., Elsevier Health Sciences, 2002.

29. Werts, William M., 'PennDOT's First FRP Deck, Polymer Composites II: Applications of Composites in Infrastructure Renewal and Economic Development, Creese, Robert C., and GangaRao, Hota ed., Organized by Constructed Facilities Center, West Virginia University, Morgantown, West Virginia, 2001.

30. Wevers, M., Verpoest, I., De Meester, P., and Aernoudt, E., "Identification of Fatigue Failure Modes in Carbon Fibre Reinforced Composites with the Energy Discriminating Acoustic Emission Method", Acoustic Emission: Current Practice and Future Directions, ASTM STP 1077, Sachse/Roget/Yamaguchi ed., pp. 416-423, 1991.

31. Yeh, Shu-Kai, and Cho, Eung Ha, personal communication, 2003. 


\section{Appendix-A}

\section{Frequency vs. Amplitude Plots}

Tension Specimens

Tests $2 \mathrm{t}-7 \mathrm{t}$

Appendix-A: Frequency vs. Amplitude Plots for Tension Specimens 

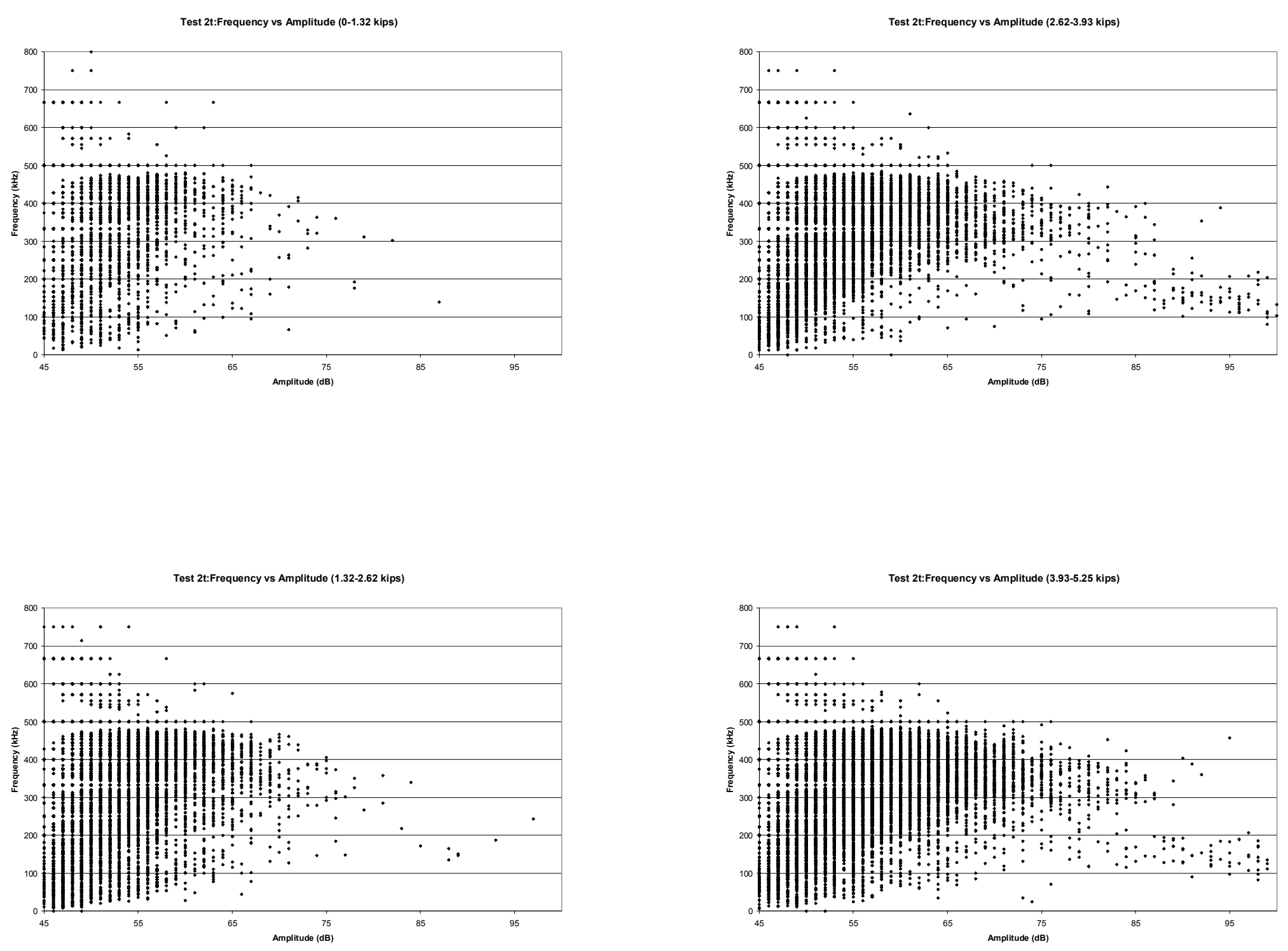

Appendix-A: Frequency vs. Amplitude Plots for Tension Specimens 

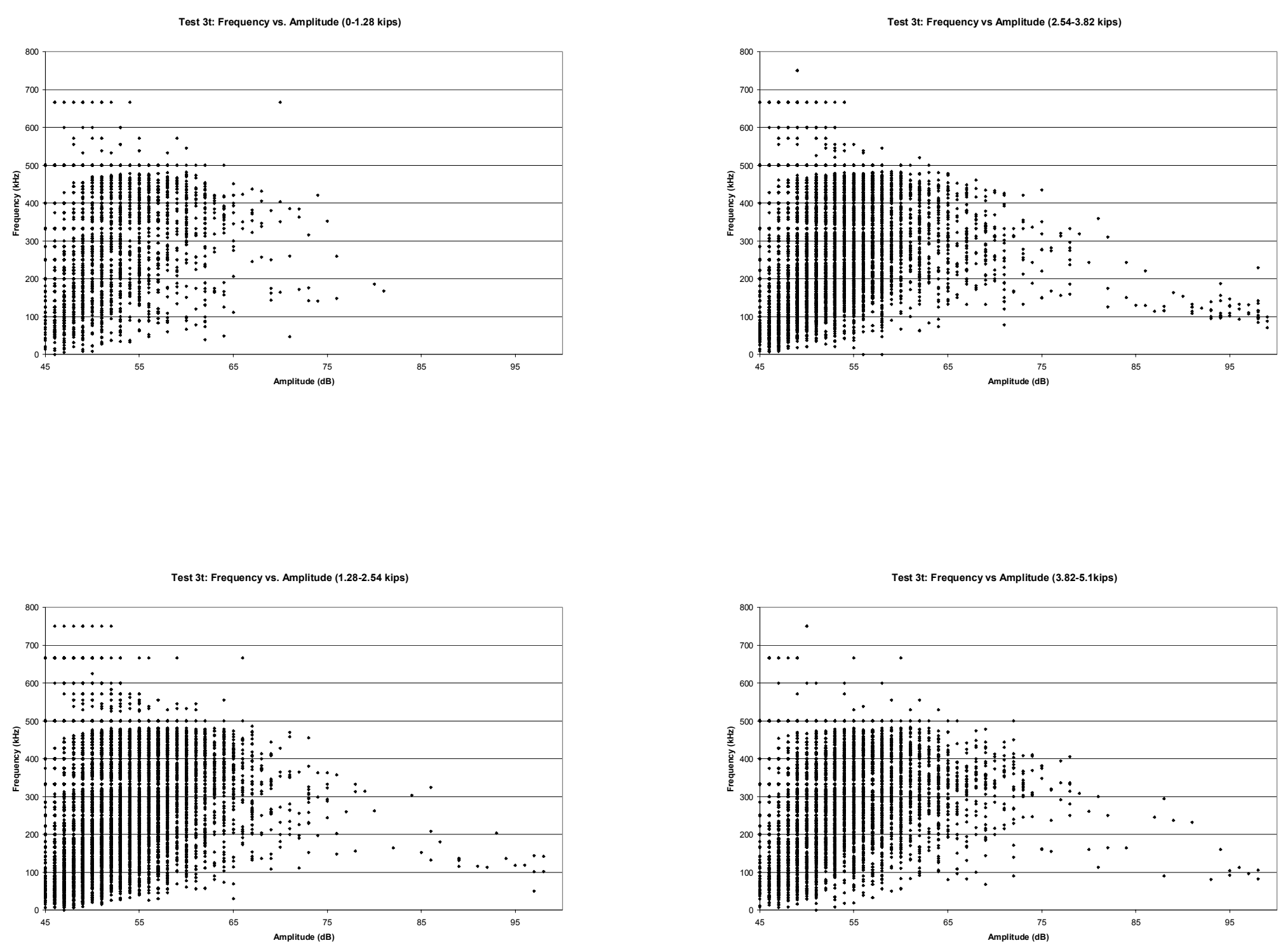

Appendix-A: Frequency vs. Amplitude Plots for Tension Specimens 

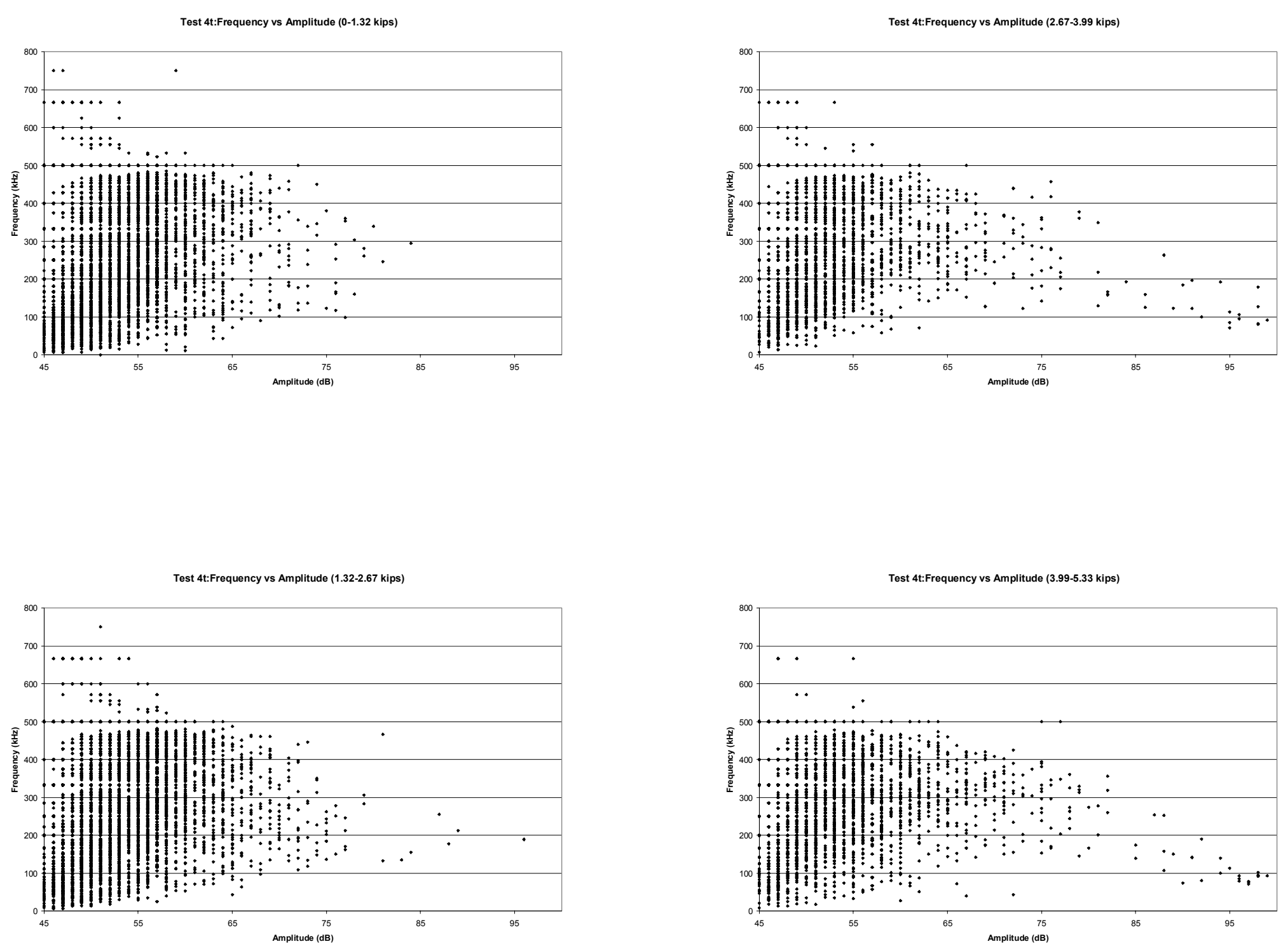

Appendix-A: Frequency vs. Amplitude Plots for Tension Specimens 

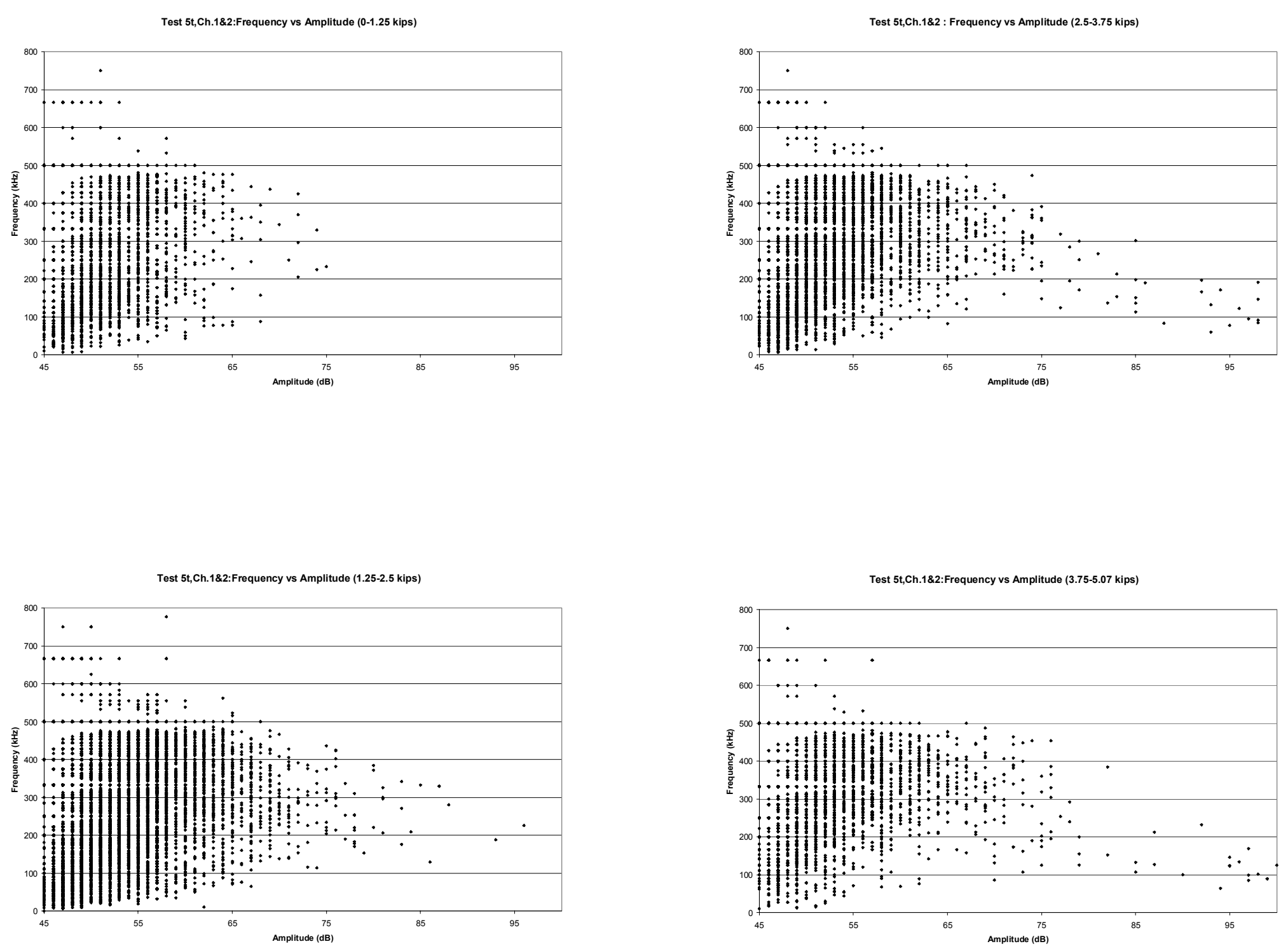

Appendix-A: Frequency vs. Amplitude Plots for Tension Specimens 
Test 6t, Ch. 182: Frequency vs Amplitude (0-1.32 kips)

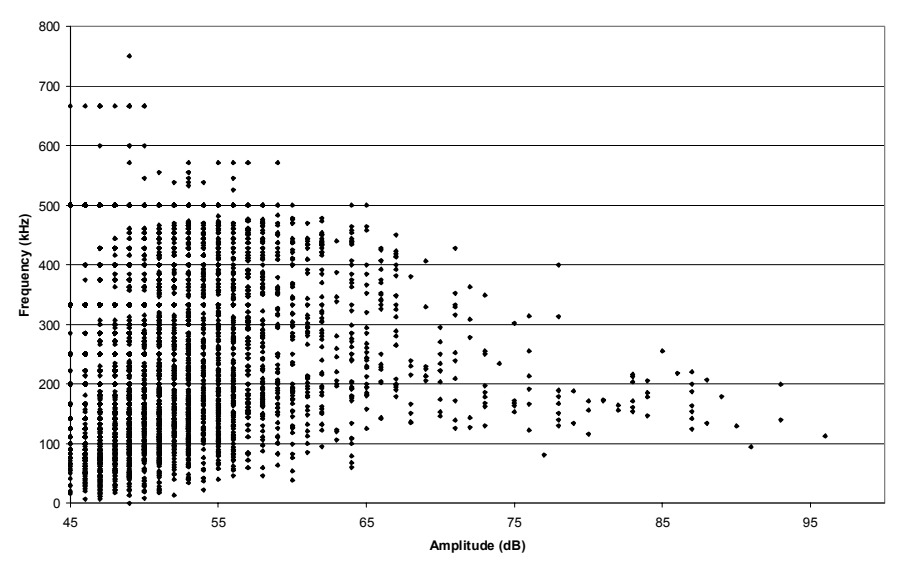

Test 6t, Ch.182:Frequency vs Amplitude (1.32-2.65 kips)

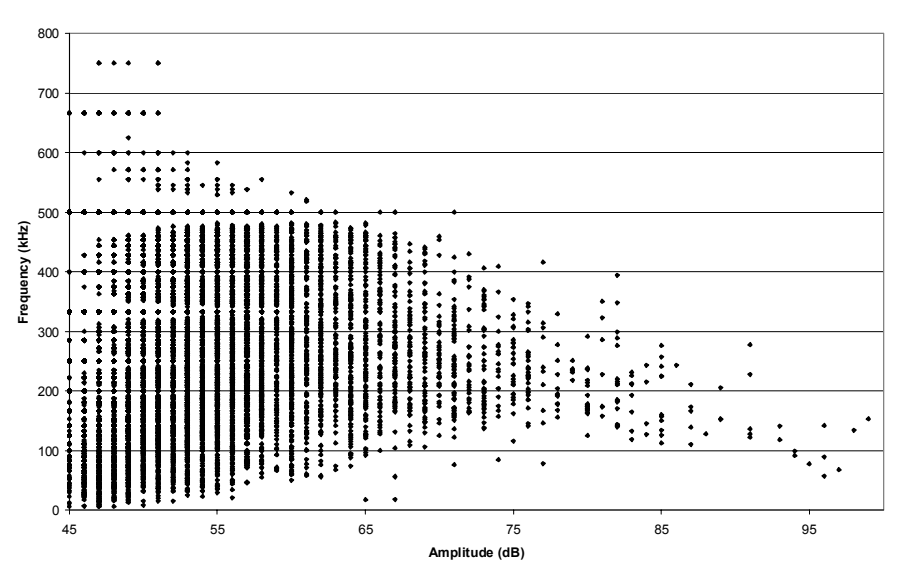

Test 6t, Ch.182:Frequency vs Amplitude (2.65-3.97 kips)

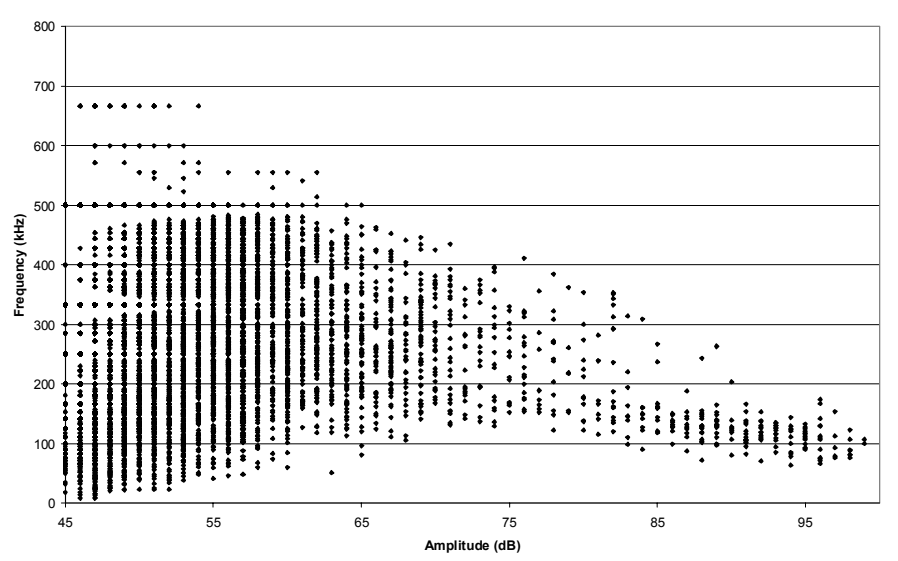

Test 6t, Ch.182:Frequency vs Amplitude (3.97-5.3 kips)

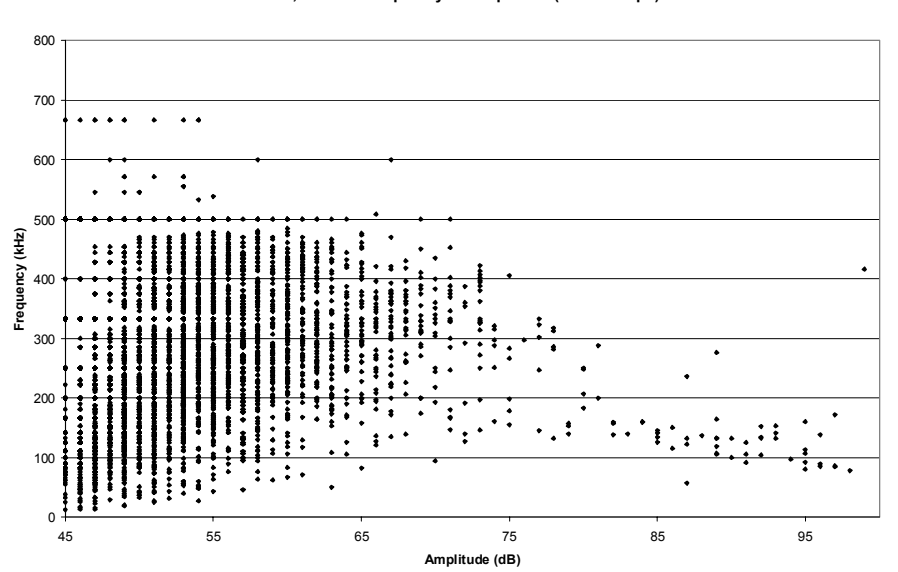

Appendix-A: Frequency vs. Amplitude Plots for Tension Specimens 
Test 7t, Ch.182: Frequency vs Amplitude (0-1.325 kips)

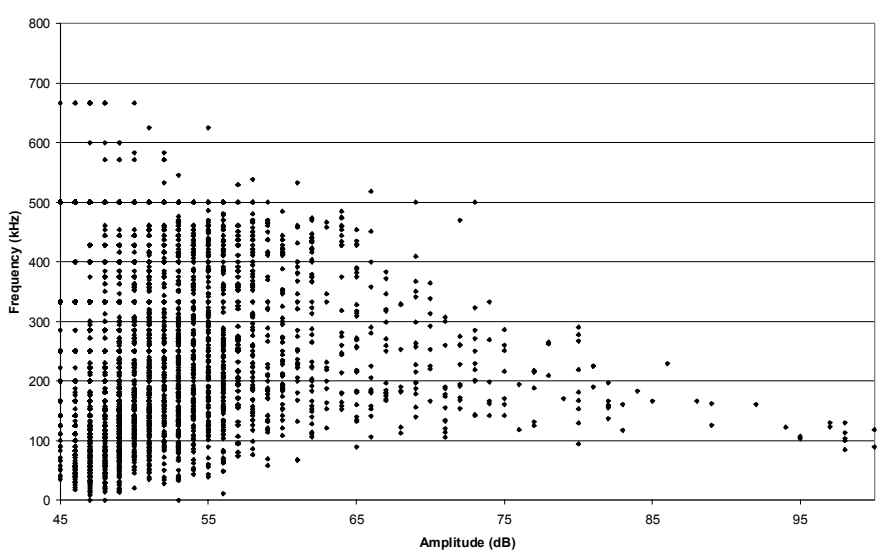

Test 7t, Ch. 182: Frequency vs Amplitude (1.325-2.65 kips)

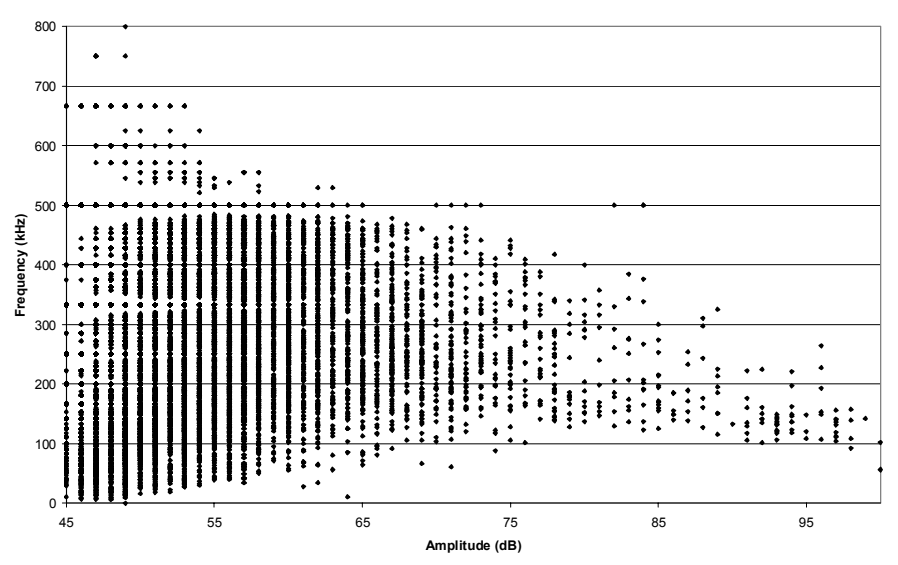

Test 7t, Ch. 182: Frequency vs Amplitude (2.65-3.98 kips)

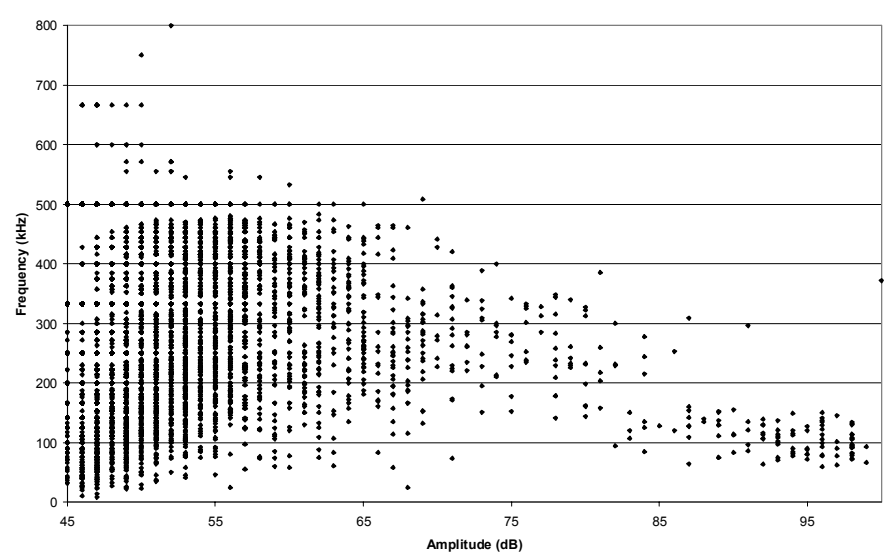

Test 7t, Ch. 182: Frequency vs Amplitude (3.98-5.3 kips)

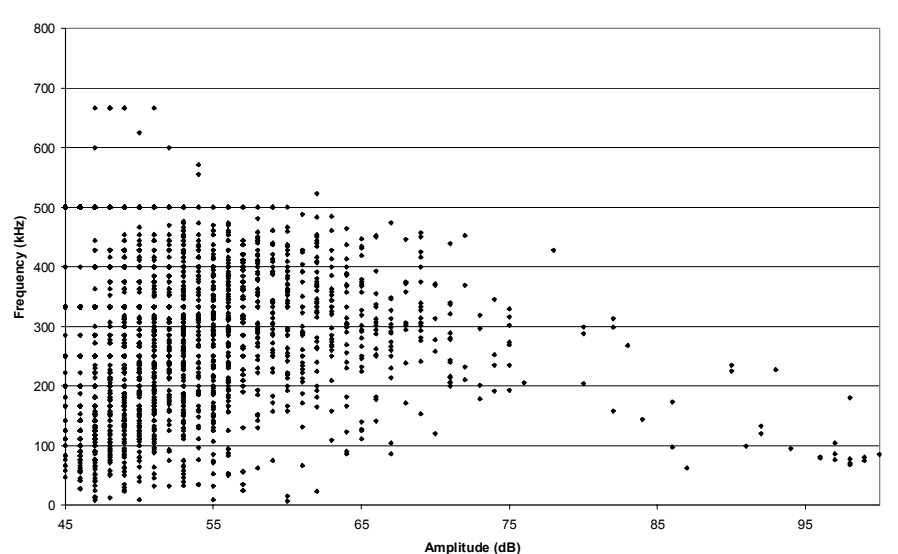

Appendix-A: Frequency vs. Amplitude Plots for Tension Specimens 


\section{Appendix-B}

\section{Amplitude vs. Duration Plots}

Tension Specimens

Tests $2 \mathrm{t}-7 \mathrm{t}$

Appendix-B: Amplitude vs. Duration Plots for Tension Specimens 
Test 2t:Amplitude vs Duration (0-1.32 kips)

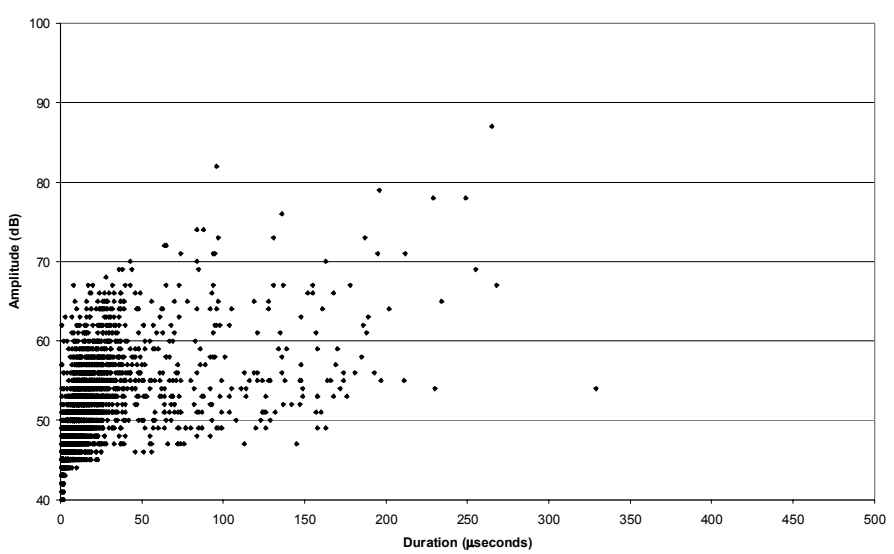

Test 2t:Amplitude vs Duration (1.32-2.62 kips)

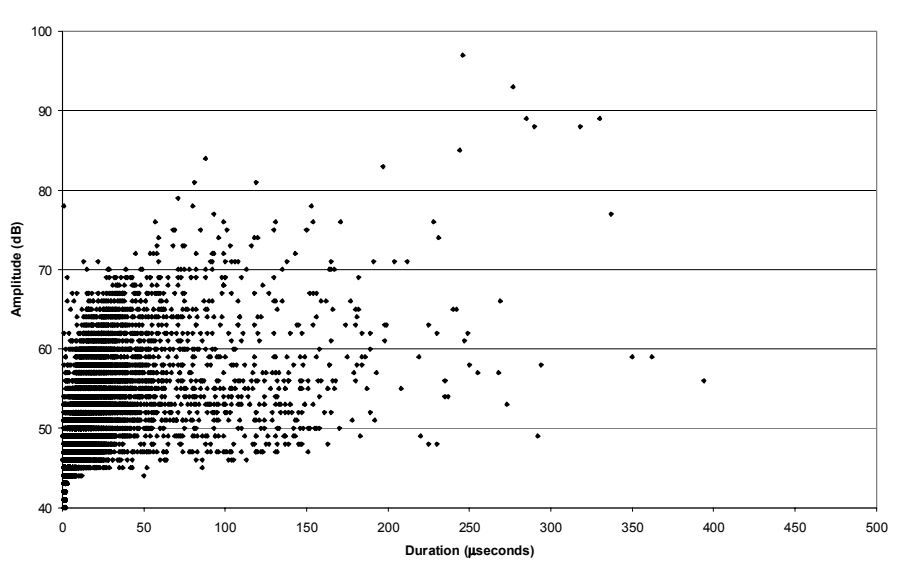

Test 2 Anpmingevs Duration (2.62-3.93 kips)

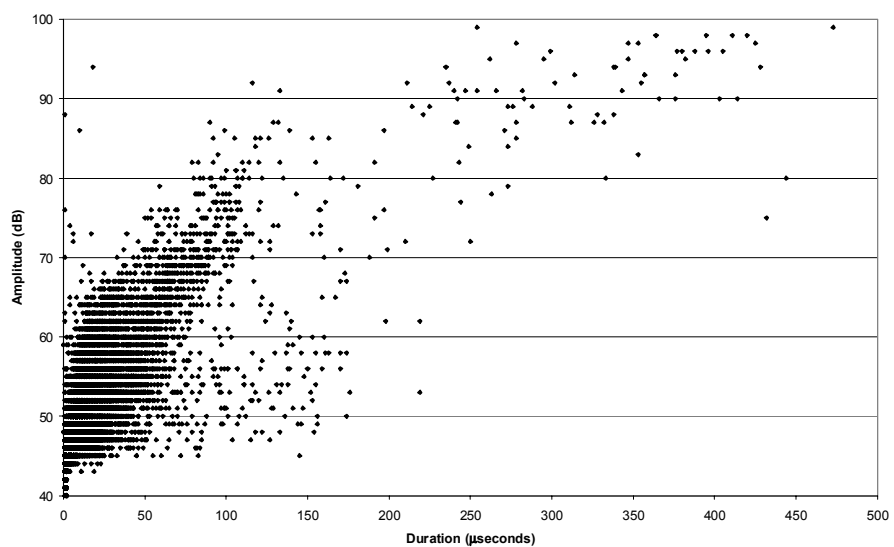

Test 2t:Amplitude vs Duration (3.93-5.25 kips)

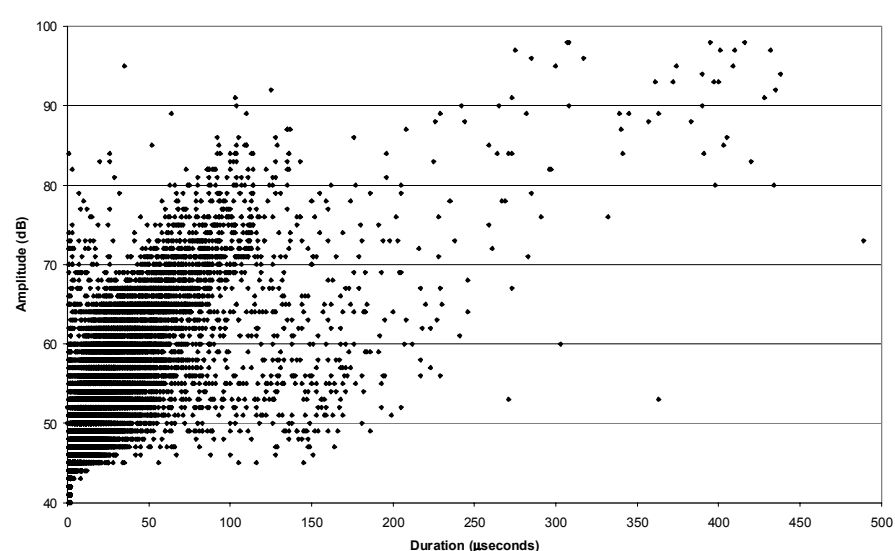

Appendix-B: Amplitude vs. Duration Plots for Tension Specimens 
Test 3t: Amplitude vs. Duration (0-1.28 kips)

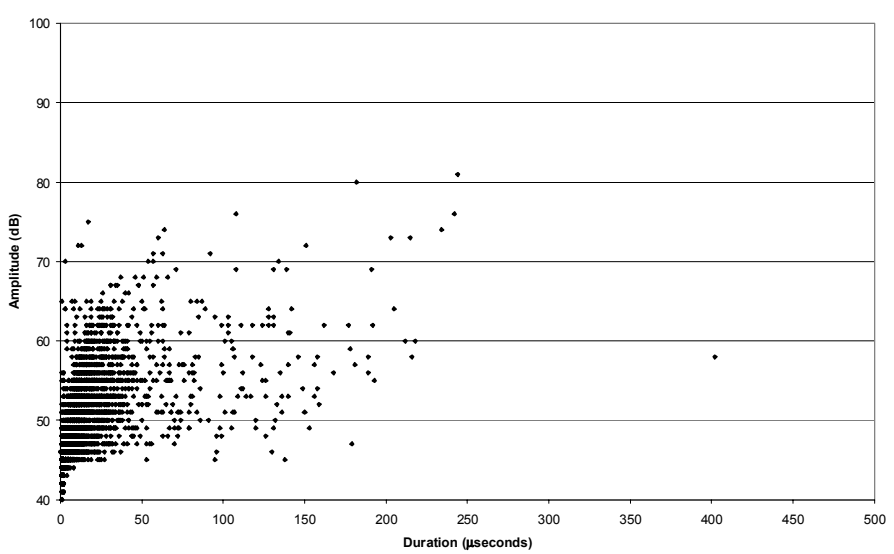

Test 3t: Amplitude vs Duration (1.28-2.54 kips)

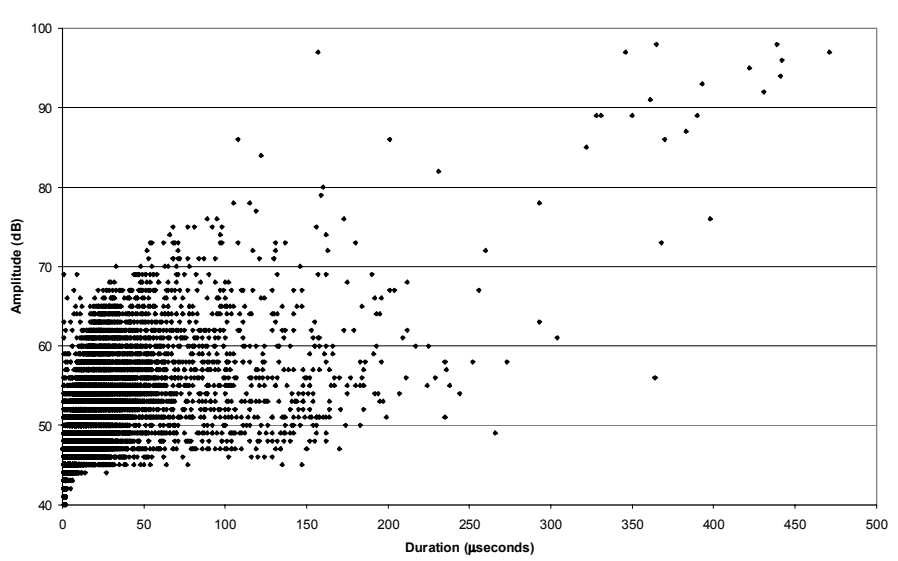

Test 3t: Amplitude vs Duration (2.54-3.82 kips)

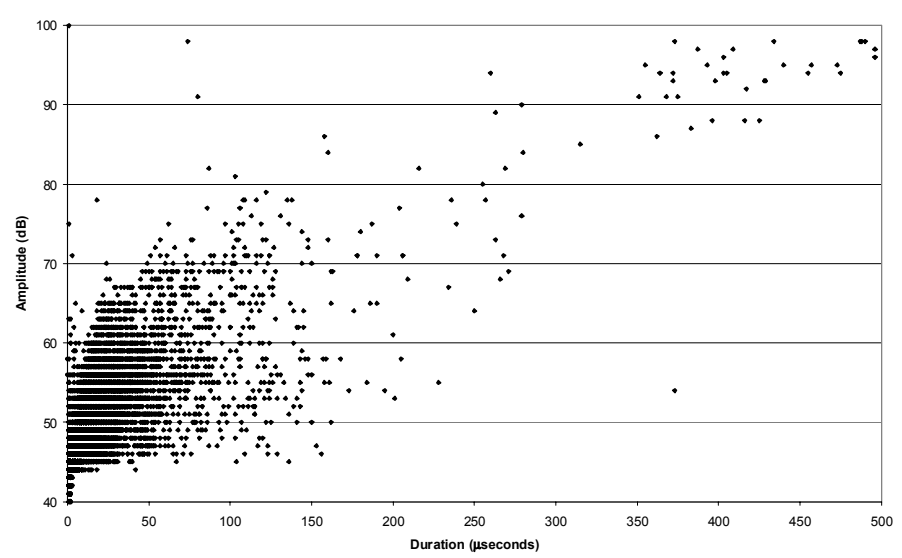

Test 3t: Amplitude vs Duration (3.82-5.1 kips)

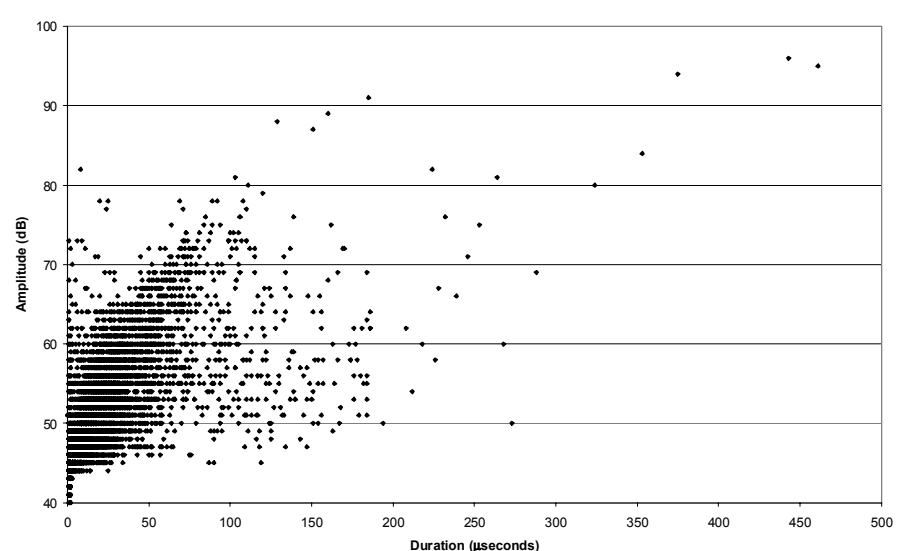

Appendix-B: Amplitude vs. Duration Plots for Tension Specimens 
Test 4t:Amplitude vs Duration (0-1.32 kips)

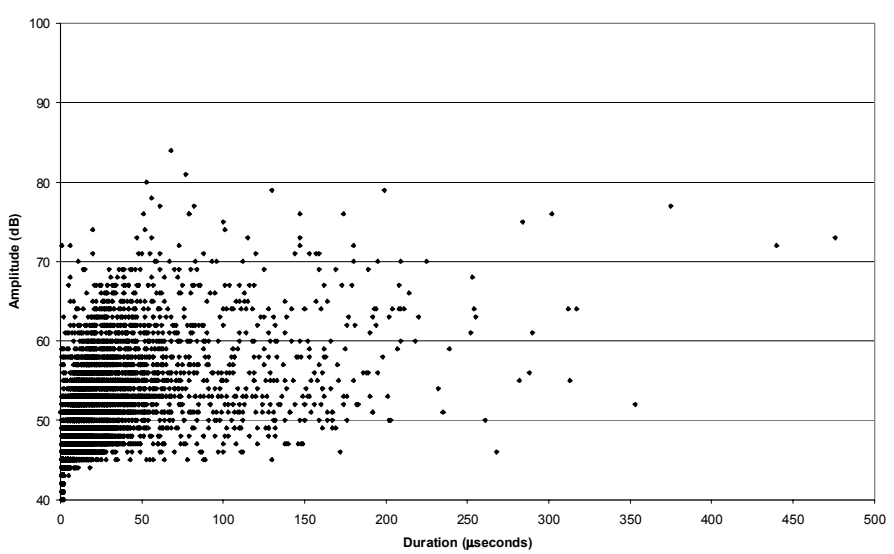

Test 4t: Amplitude vs Duration (1.32-2.67 kips)

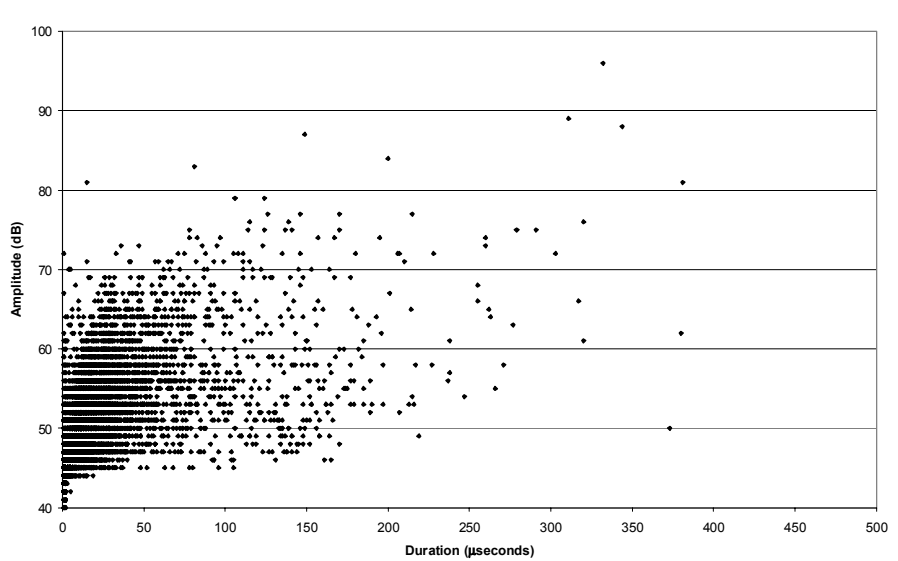

Test 4t:Amplitude vs Duration (2.67-3.99 kips)

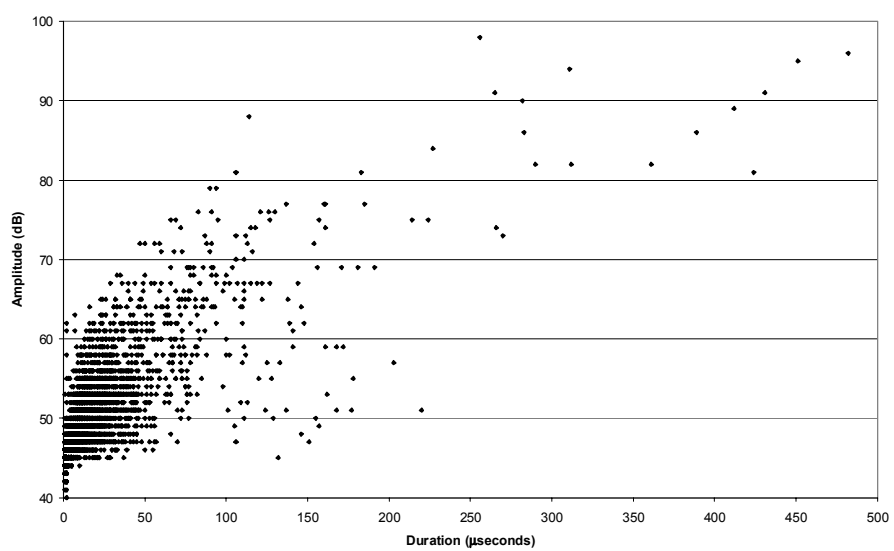

Test 4t:Amplitude vs Duration (3.99-5.33 kips)

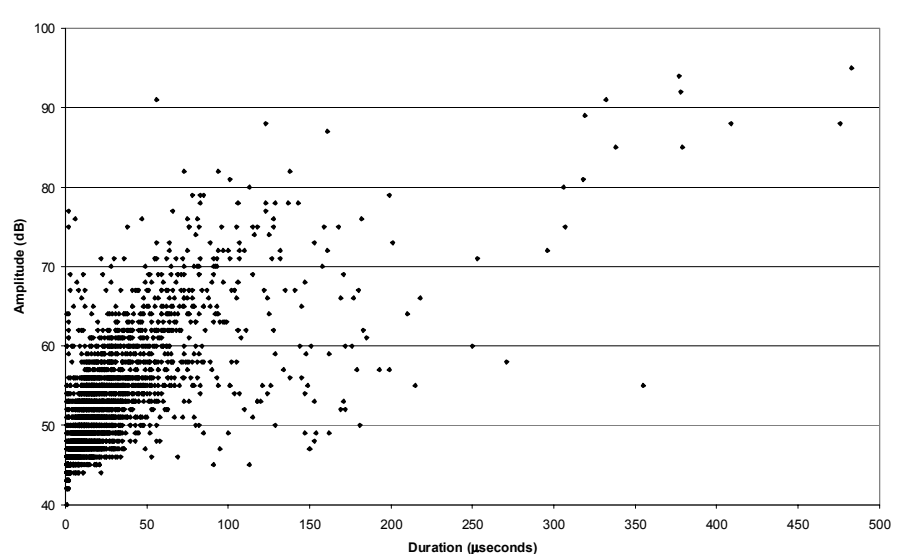

Appendix-B: Amplitude vs. Duration Plots for Tension Specimens 
Test 5t,Ch.182:Amplitude vs Duration (0-1.25 kips)

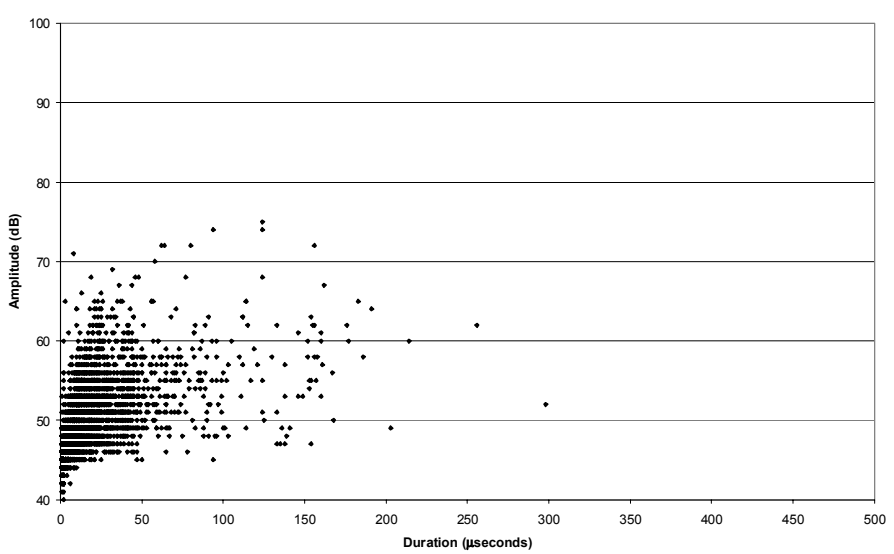

Test 5t,Ch.182:Amplitude vs Duration (1.25-2.5 kips)

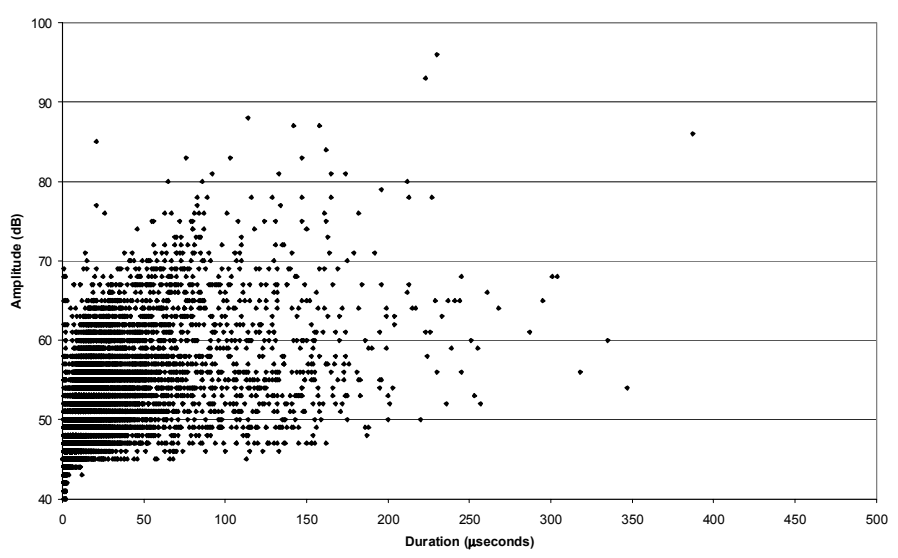

Test 5t,Ch.182:Amplitude vs Duration (2.5-3.75 kips)

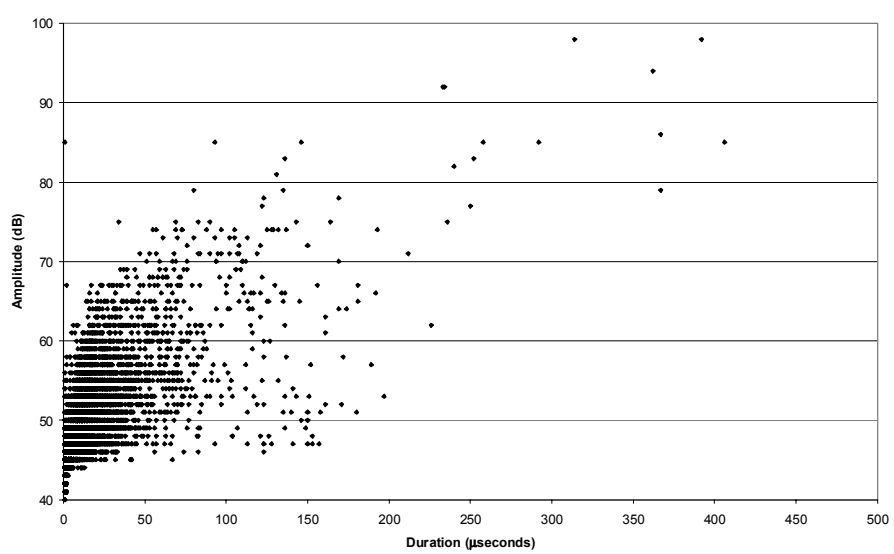

Test 5t,Ch.122:Amplitude vs Duration (3.75-5.1 kips)

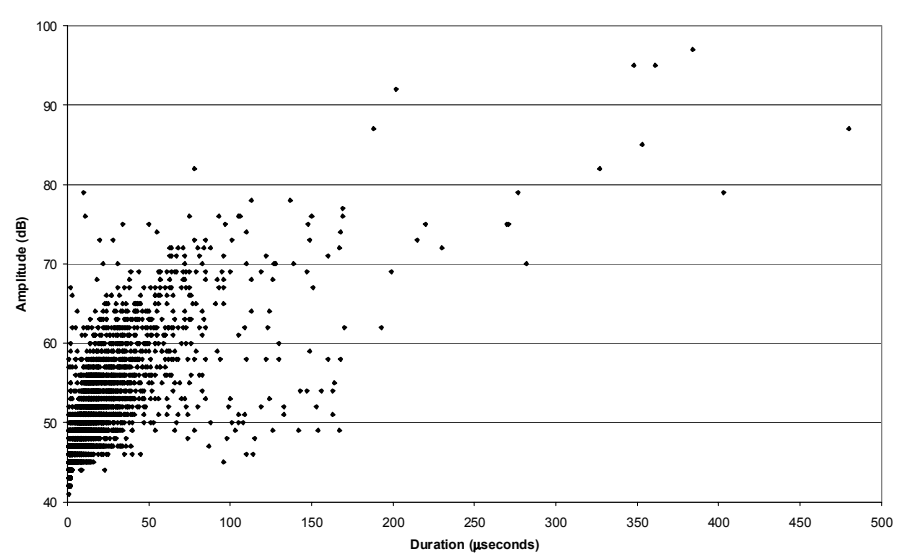

Appendix-B: Amplitude vs. Duration Plots for Tension Specimens 
Test 6t,Ch.122:Amplitude vs Duration (0-1.32 kips)

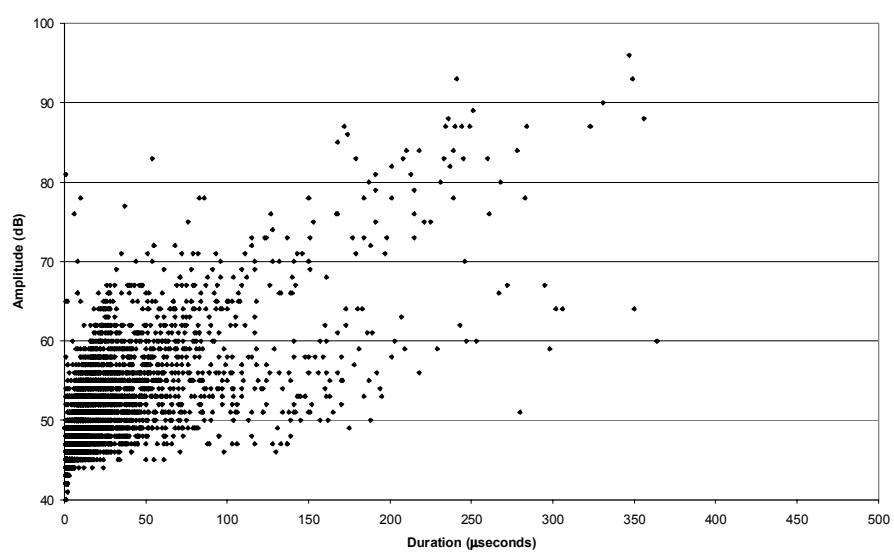

Test 6t,Ch.182:Amplitude vs Duration (1.32-2.65 kips)

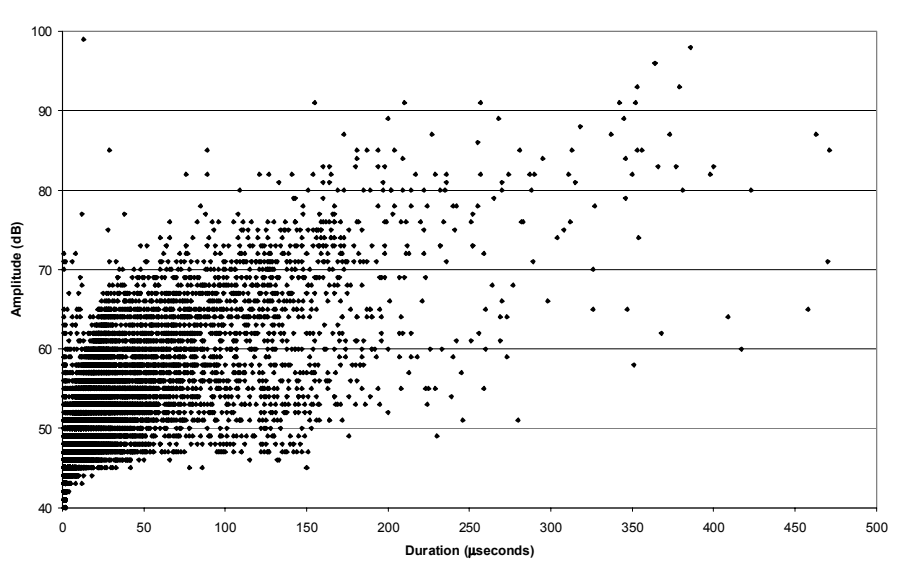

Test 6t,Ch.182:Amplitude vs Duration (2.65-3.97 kips)

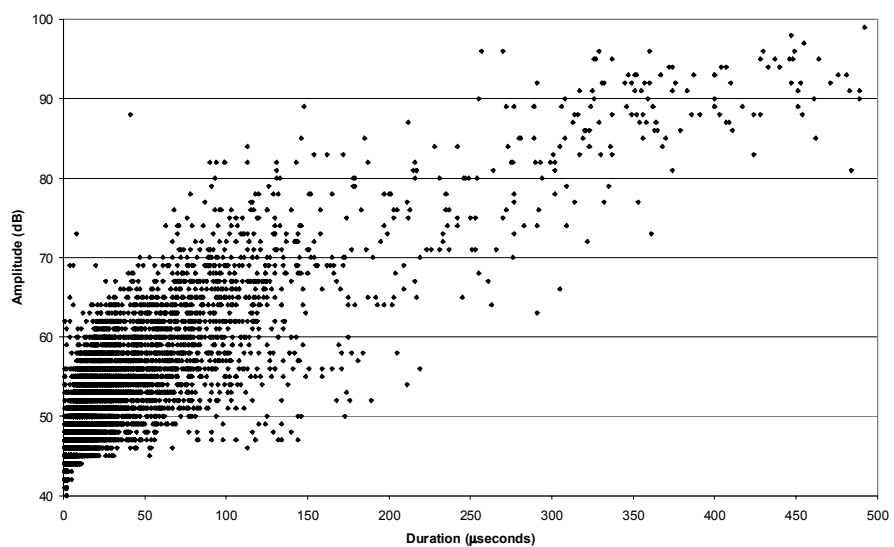

Test 6t,Ch.182:Amplitude vs Duration (3.97-5.3 kips)

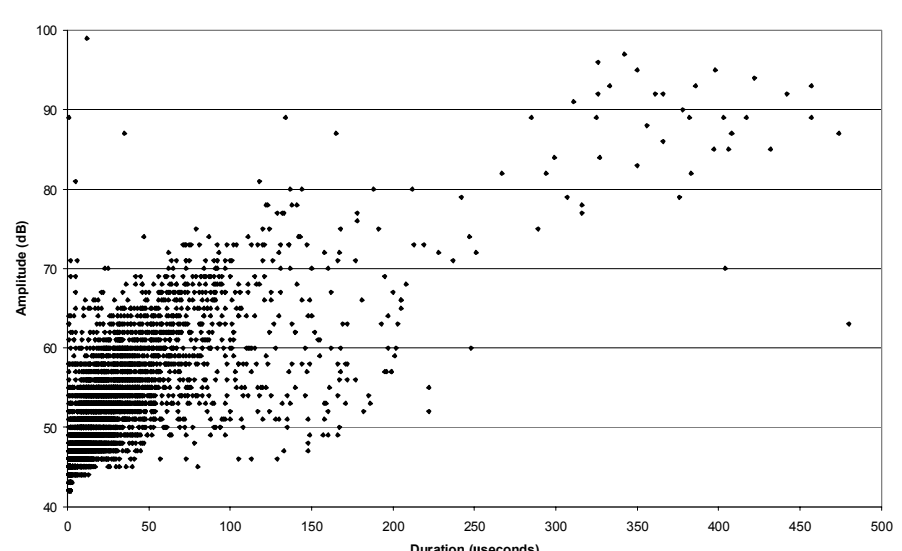

Appendix-B: Amplitude vs. Duration Plots for Tension Specimens 
Test 7t, Ch. 182: Amplitude vs Duration (0-1.325 kips)

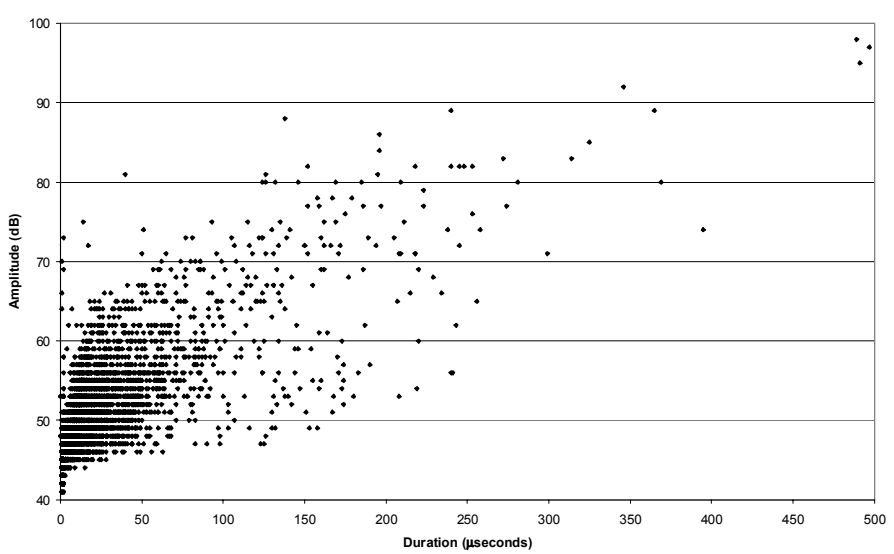

Test 7t, Ch. 182: Amplitude vs Duration (1.325-2.65 kips)

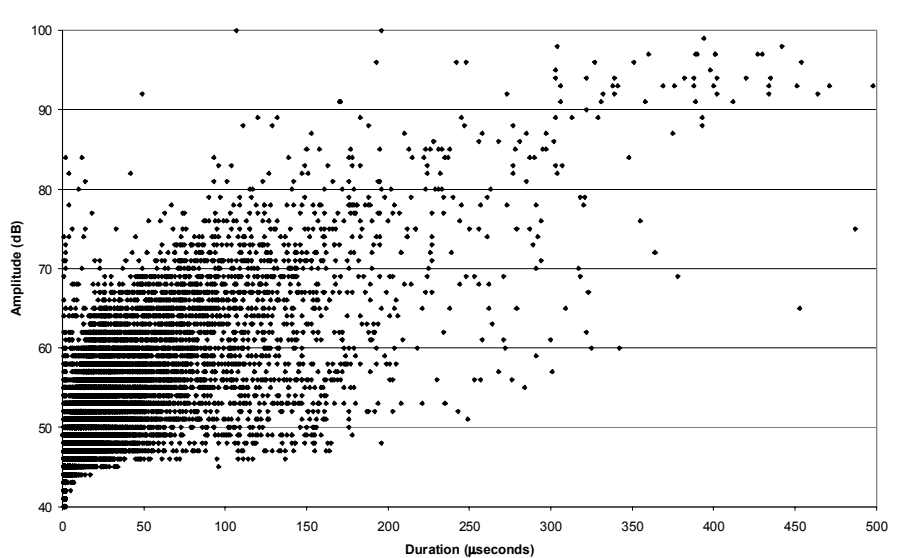

Test 7t, Ch. 182: Amplitude vs Duration (2.65-3.98 kips)

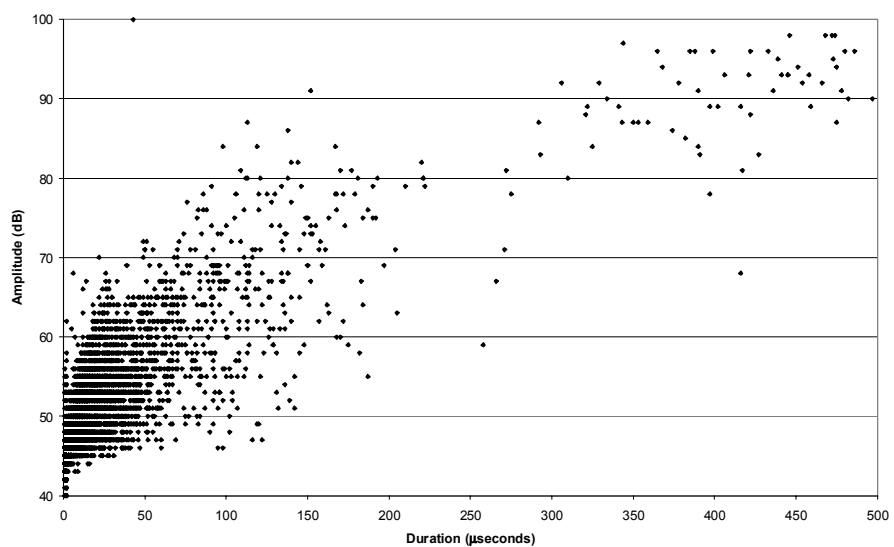

Test 7t, Ch. 182: Amplitude vs Duration (3.98-5.3 kips)

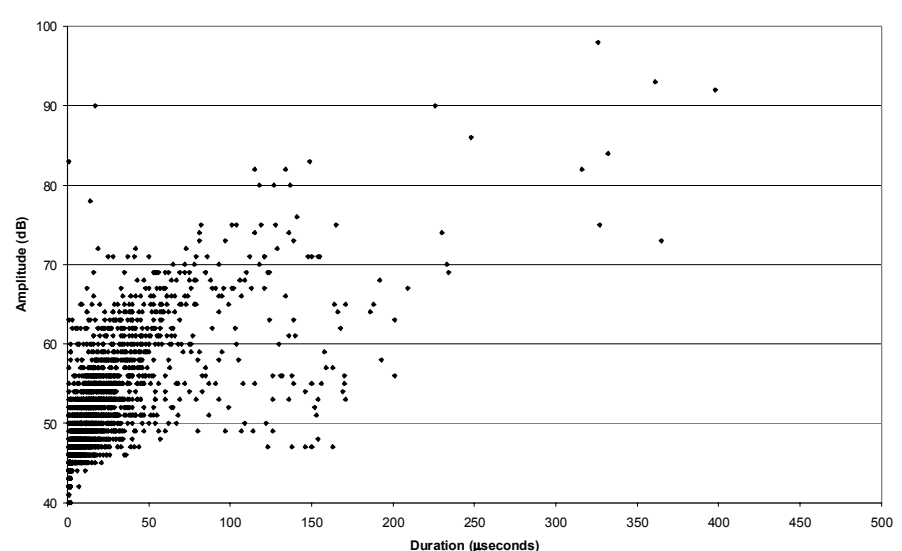

Appendix-B: Amplitude vs. Duration Plots for Tension Specimens 


\section{Appendix-C}

\section{¿Energy vs. Load Plots}

Tension Specimens

Tests $2 \mathrm{t}-4 \mathrm{t}, 6 \mathrm{t}-7 \mathrm{t}$

Appendix-C: $\Sigma$ Energy vs. Load Plots for Tension Specimens 
Test 2t: EEnergy vs Load

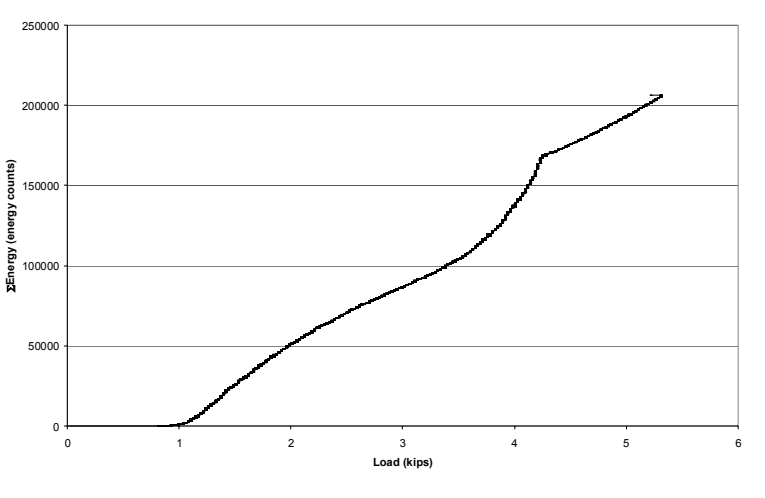

Test 3t: EEnergy vs Load

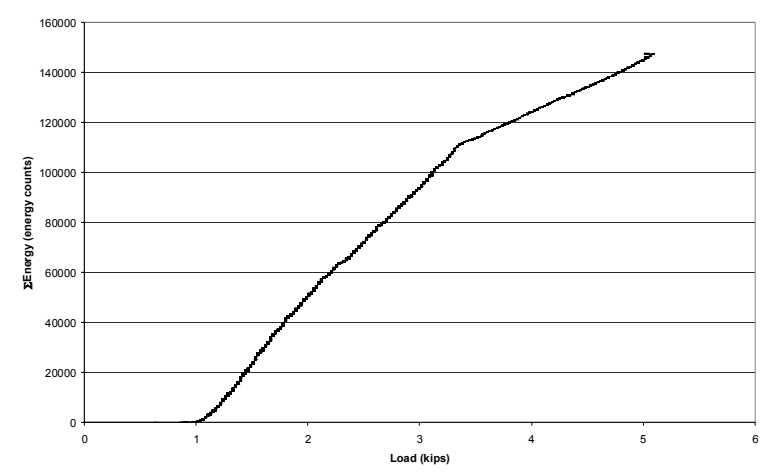

Test 7t, Ch.182: EEnergy vs Load

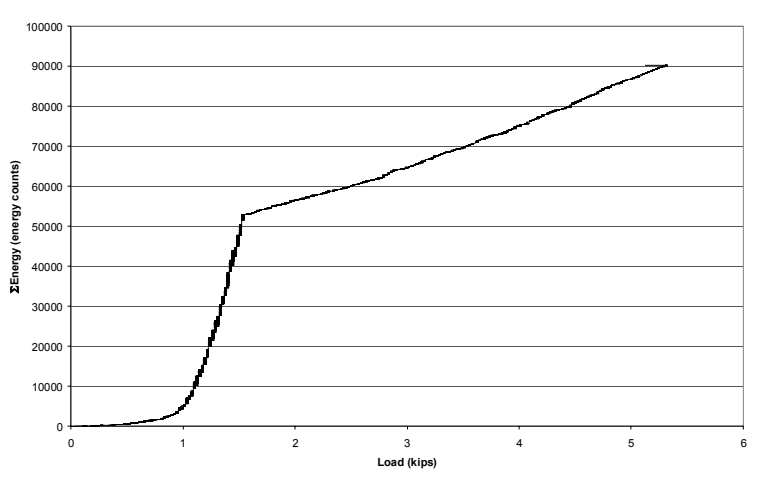

Test 6t, ch. 182: EEnergy vs. Load

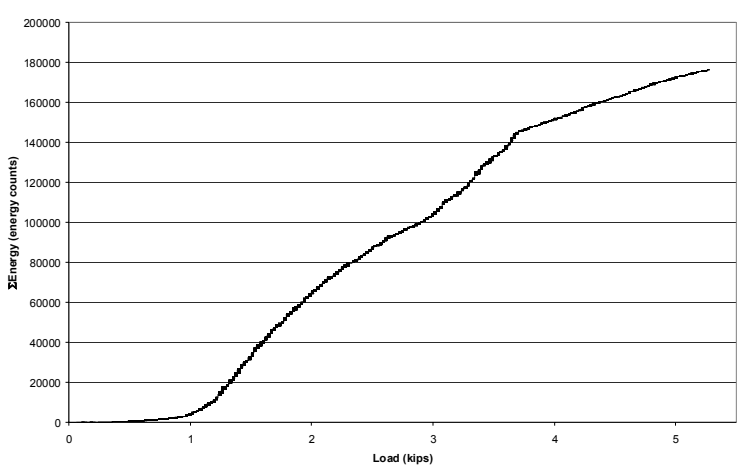

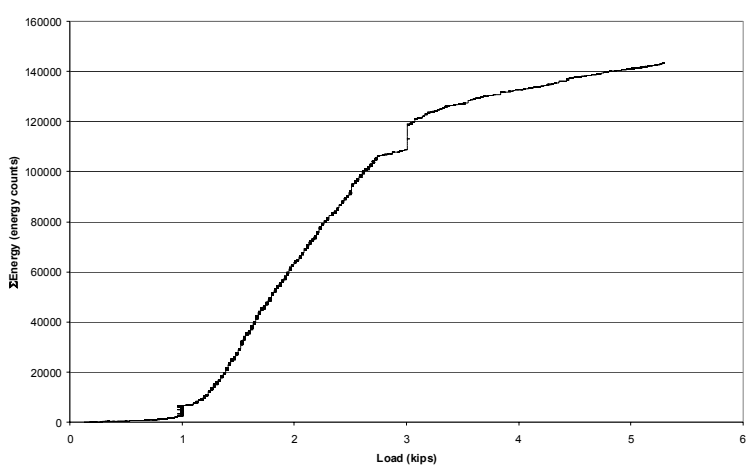

Appendix-C: $\Sigma$ Energy vs. Load Plots for Tension Specimens 


\section{Appendix-D}

Frequency vs. Amplitude Plots

Bending Specimens

Tests B1, B3-B5, B6, B8-B10, C1, C3-C5

Appendix-D: Frequency vs. Amplitude Plots for Bending Specimens 
Test B1, ch 182: Frequency vs Amplitude (1st quarter)

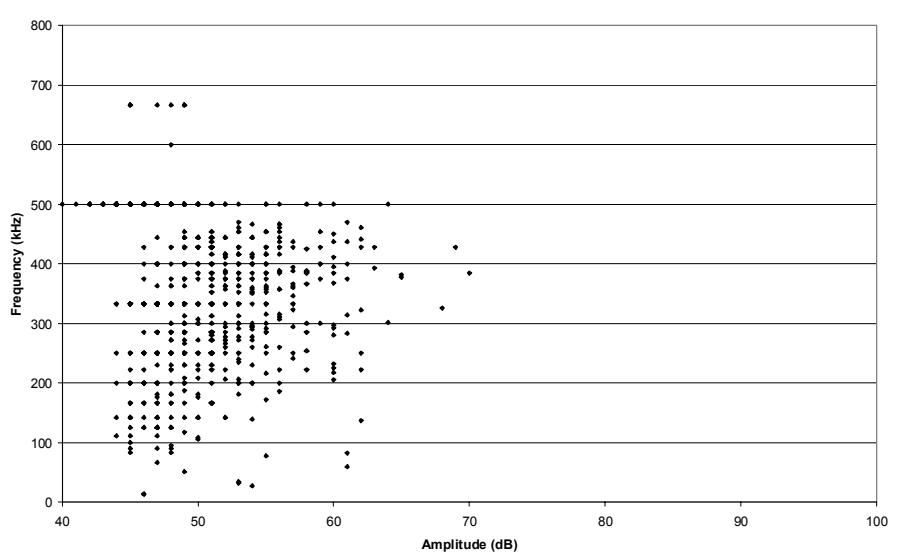

Test B1, ch 182: Frequency vs Amplitude (2nd quarter)

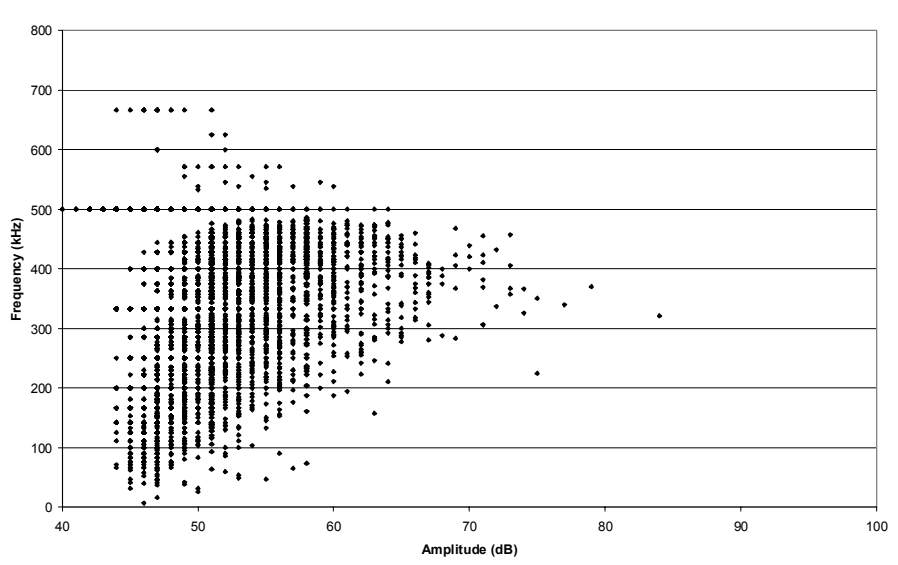

Test B1, ch 182: Frequency vs Amplitude (3rd quarter truncated)

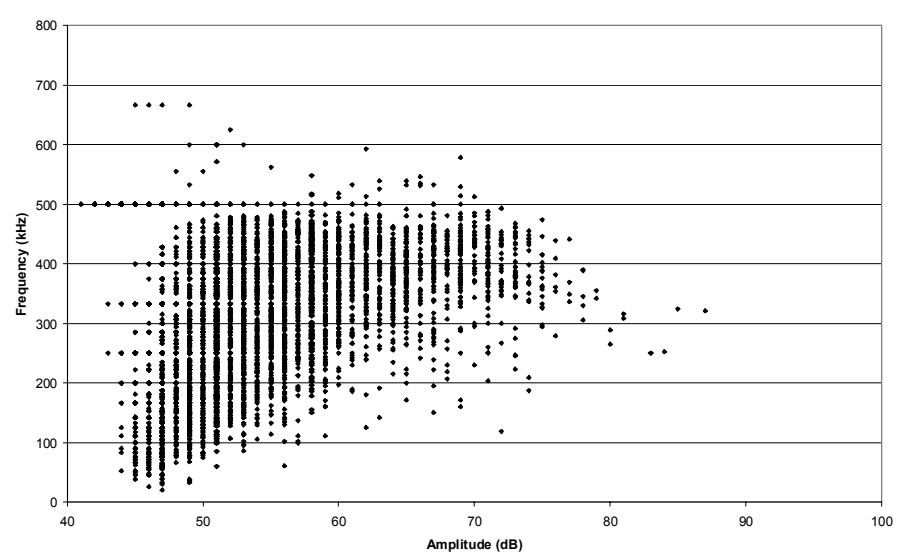

Appendix-D: Frequency vs. Amplitude Plots for Bending Specimens 
Test B3, ch 182: Frequency vs Amplitude (1st quarter)

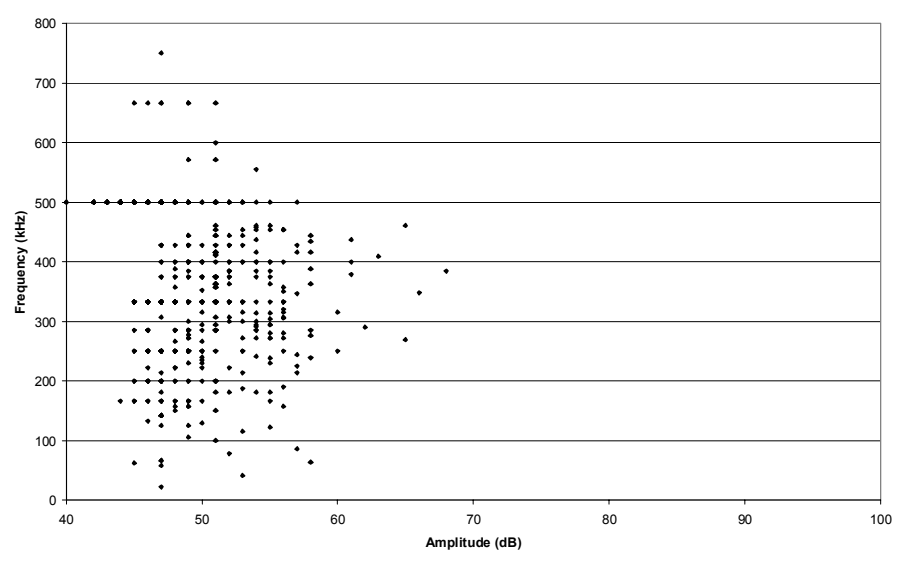

Test B3, ch 182: Frequency vs Amplitude (2nd quarter)

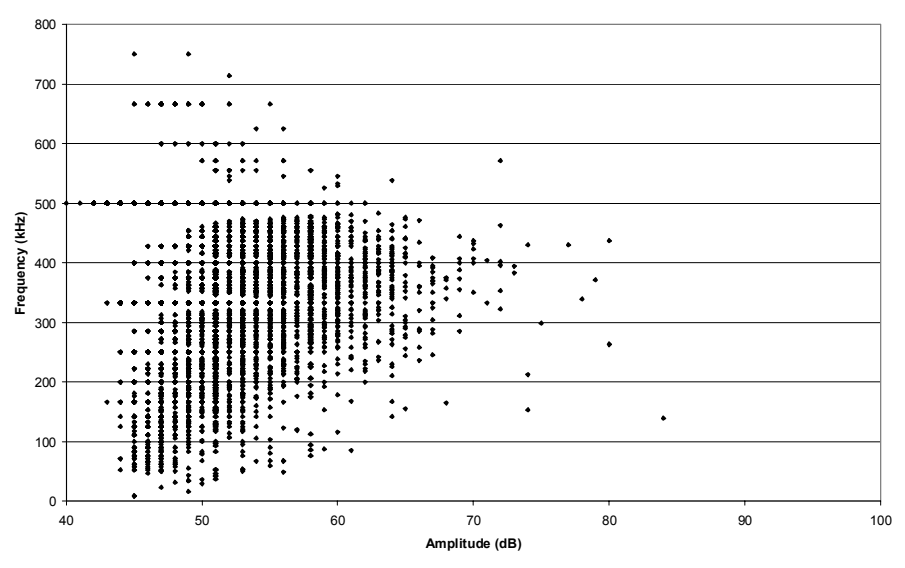

Test B3, ch 182: Frequency vs Amplitude (3rd quarter)

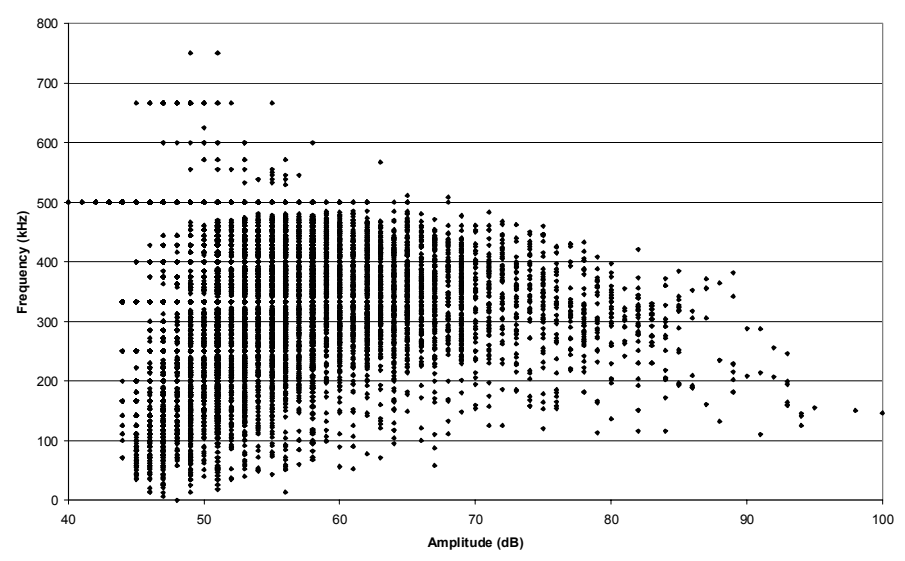

Test B3, ch 182: Frequency vs Amplitude (4th quarter)

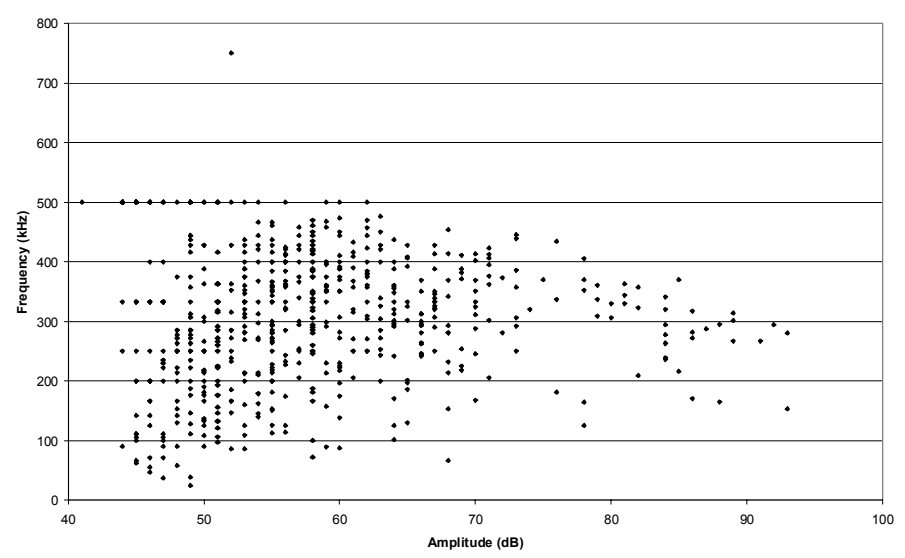

Appendix-D: Frequency vs. Amplitude Plots for Bending Specimens 
Test B4, ch 182: Frequency vs Amplitude (1st quarter)

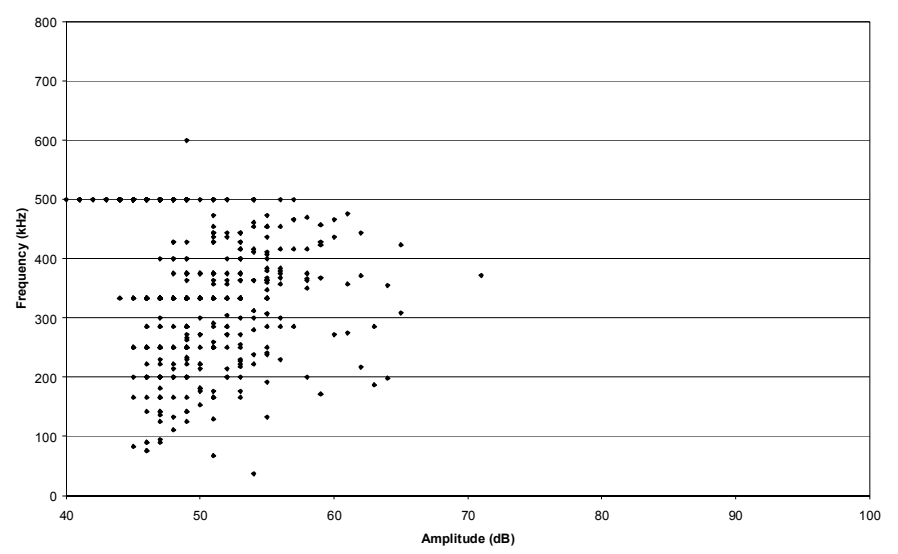

Test B4, ch 182: Frequency vs Amplitude (2nd quarter)

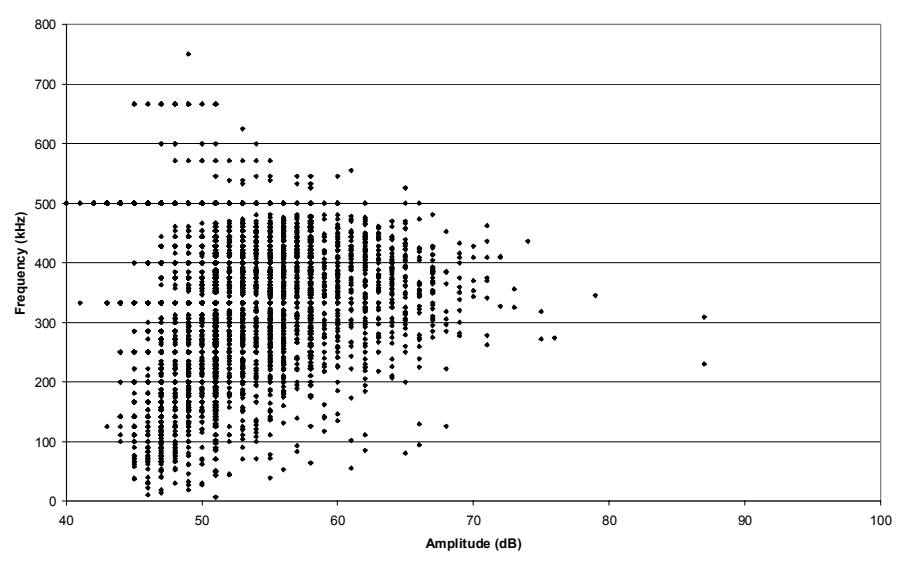

Test B4, ch 182: Frequency vs Amplitude (3rd quarter)

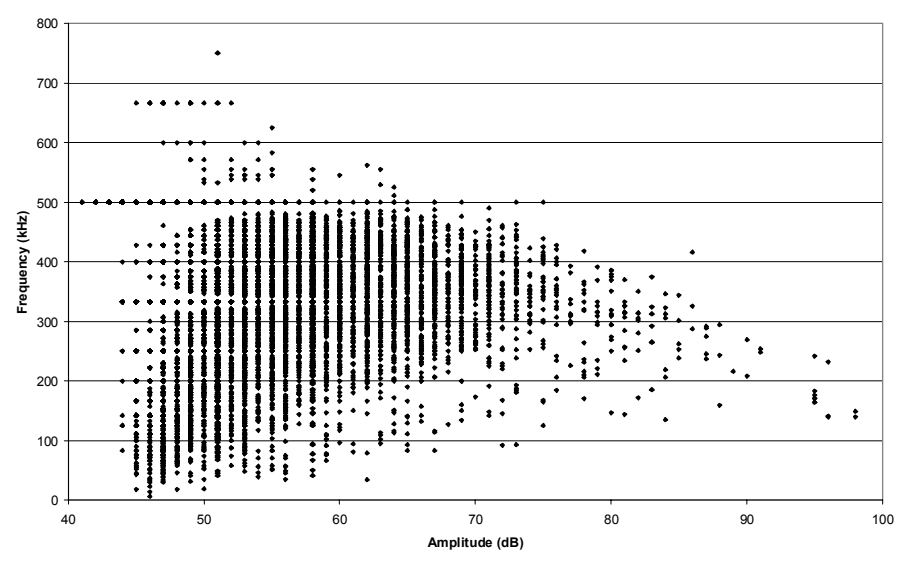

Test B4, ch 182: Frequency vs Amplitude (4th quarter)

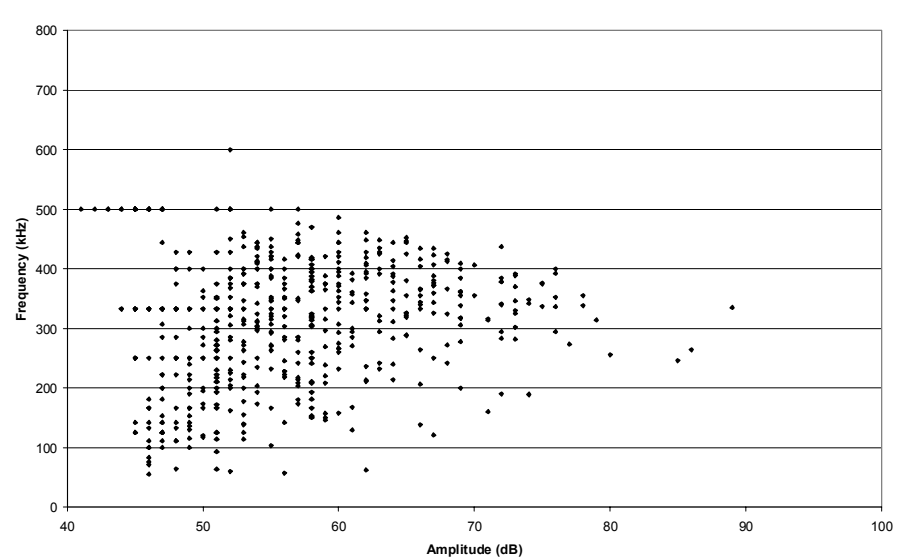

Appendix-D: Frequency vs. Amplitude Plots for Bending Specimens 
Test B5,Ch 182,: Frequency vs. Amplitude (1st quarter)

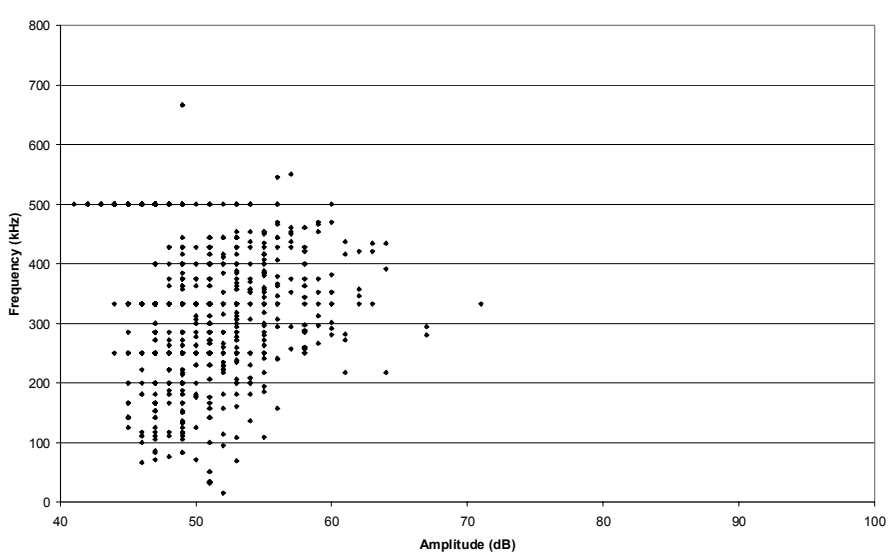

Test B5, ch 182: Frequency vs. Amplitude (2nd quarter)

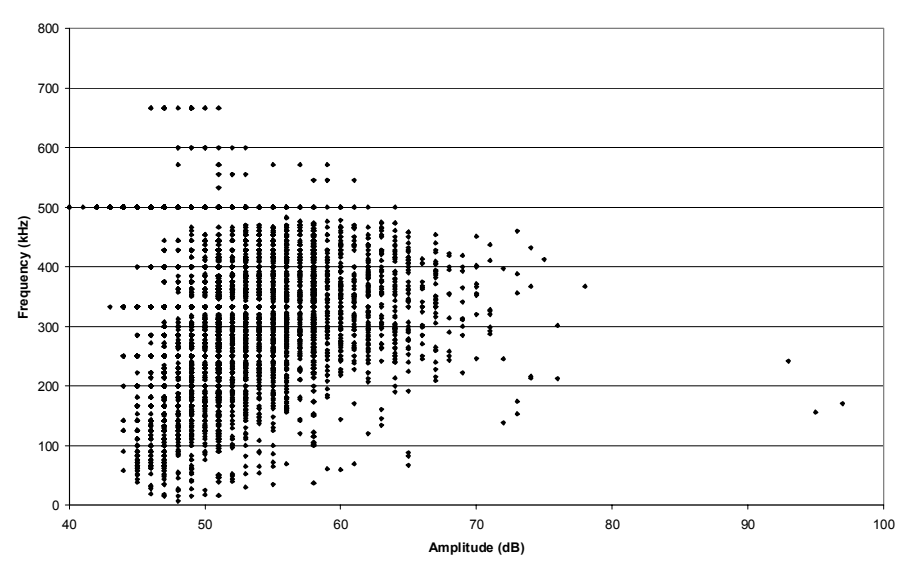

Test B5, ch 182: Frequency vs Amplitude (3rd quarter)

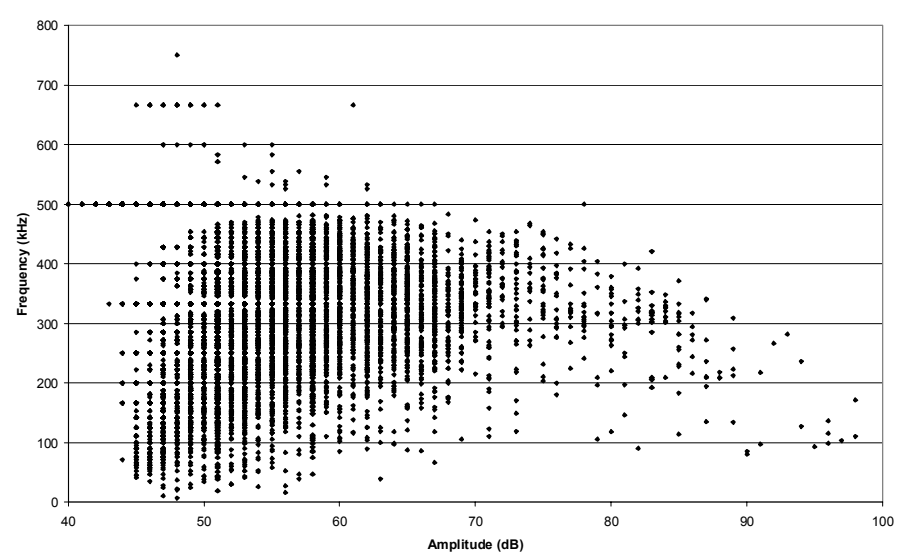

Test B5, ch 182: Frequency vs Amplitude (4th quarter)

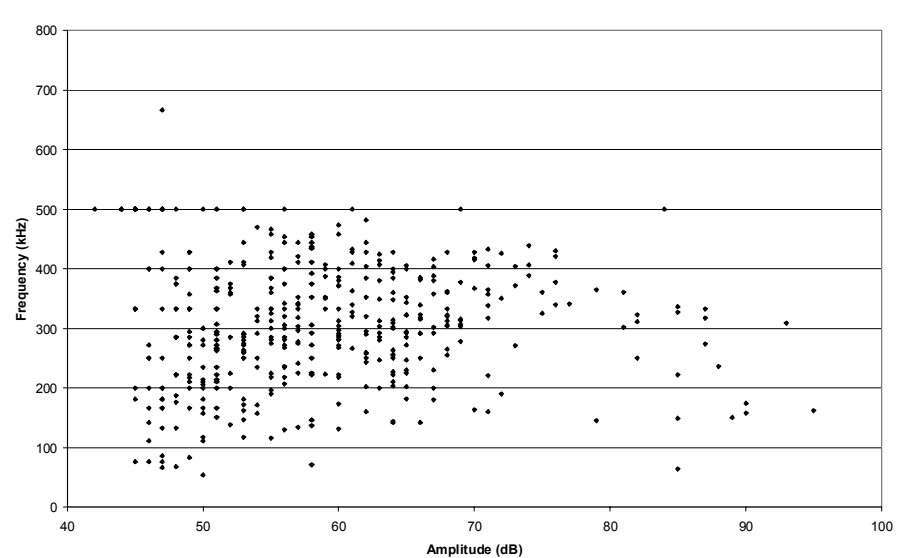

Appendix-D: Frequency vs. Amplitude Plots for Bending Specimens 

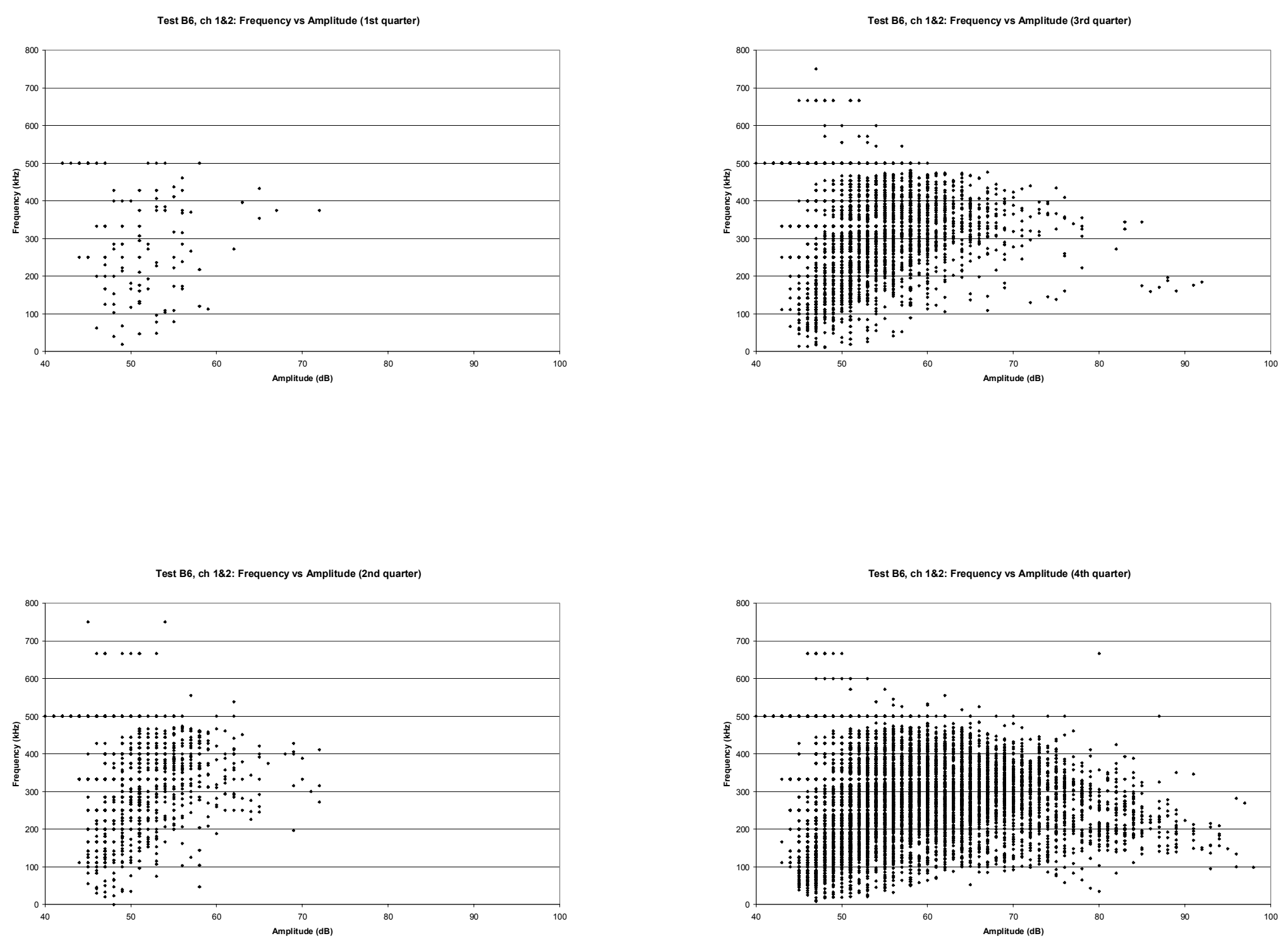

Appendix-D: Frequency vs. Amplitude Plots for Bending Specimens 

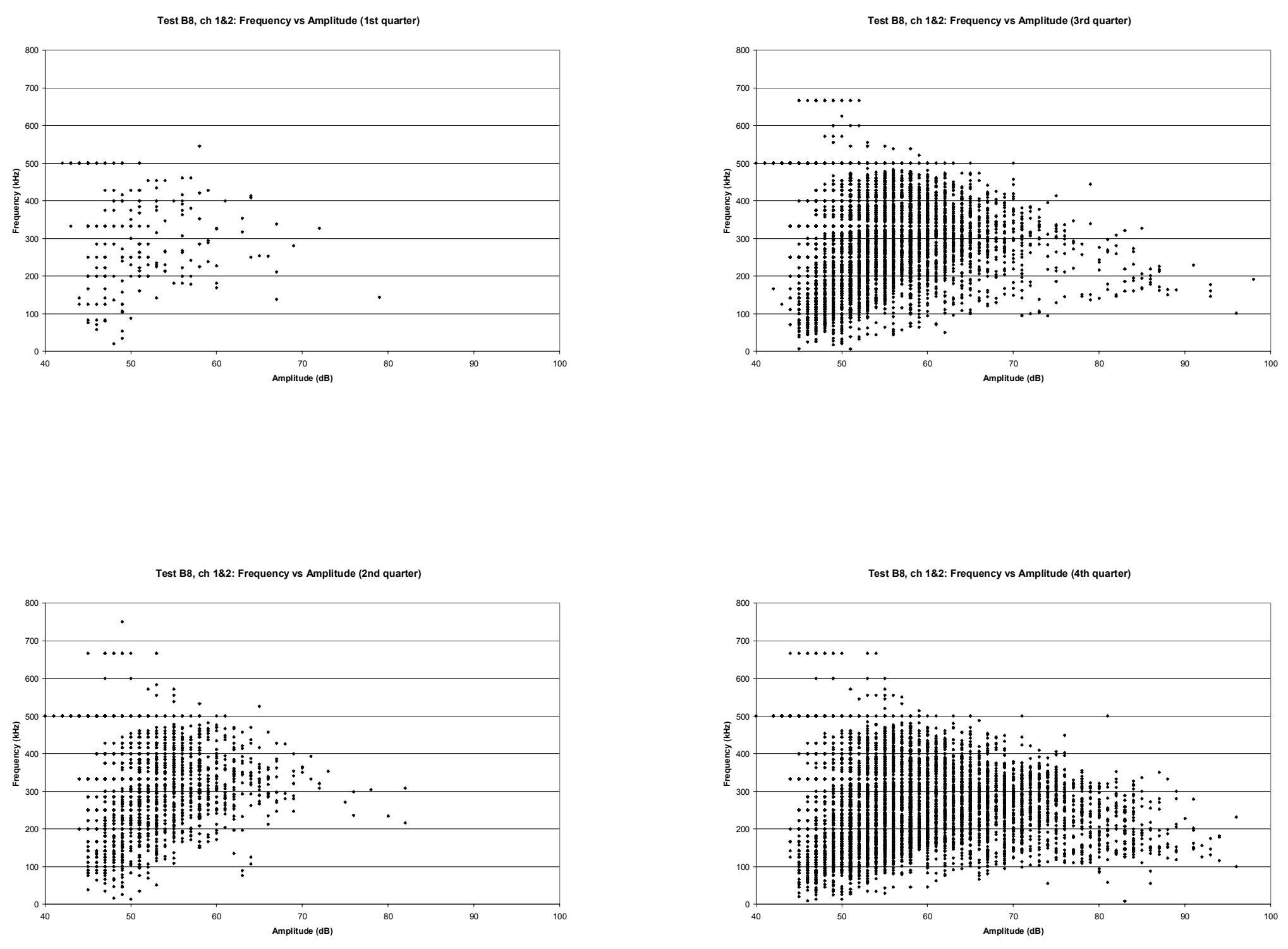

Appendix-D: Frequency vs. Amplitude Plots for Bending Specimens 
Test B9, ch 182: Frequency vs Amplitude (1st quarter)

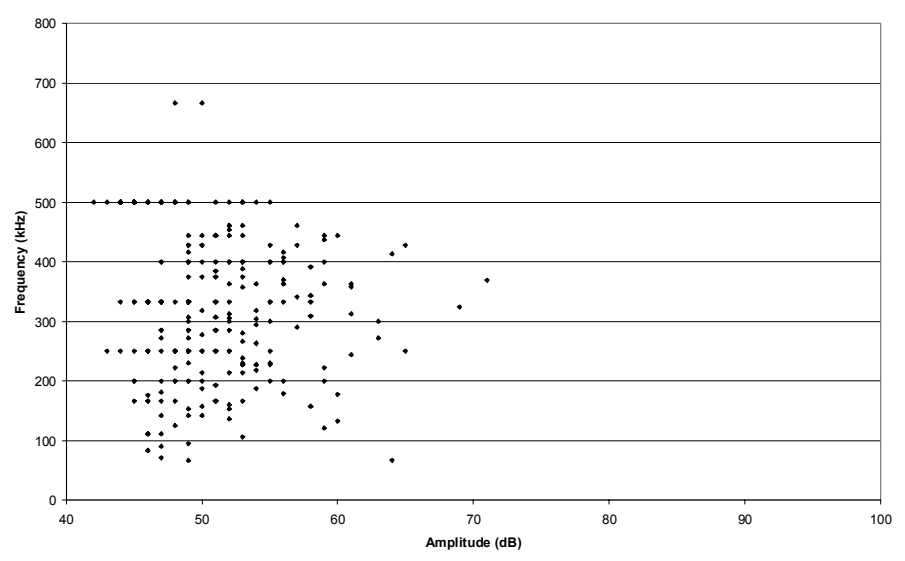

Test B9, ch 182: Frequency vs Amplitude (2nd quarter)

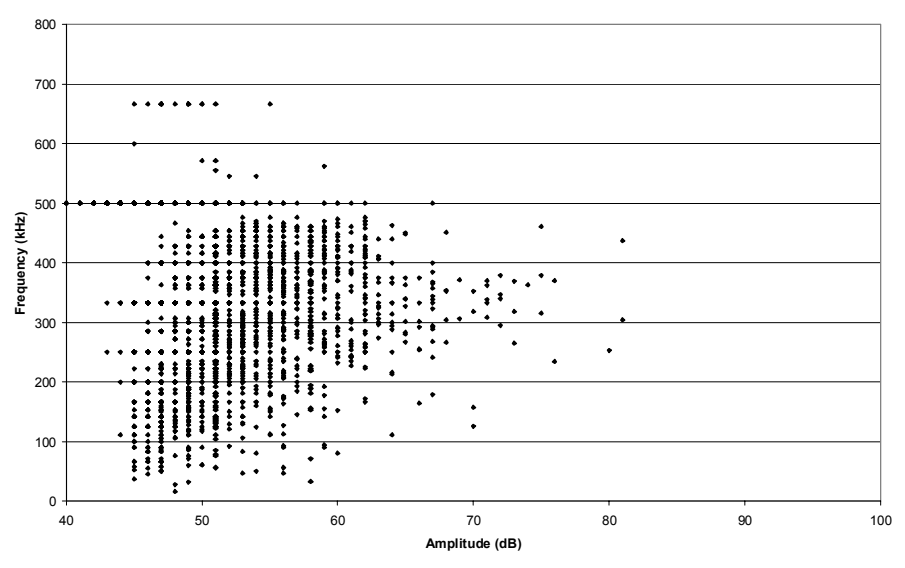

Test B9, ch 182: Frequency vs Amplitude (3rd quarter)

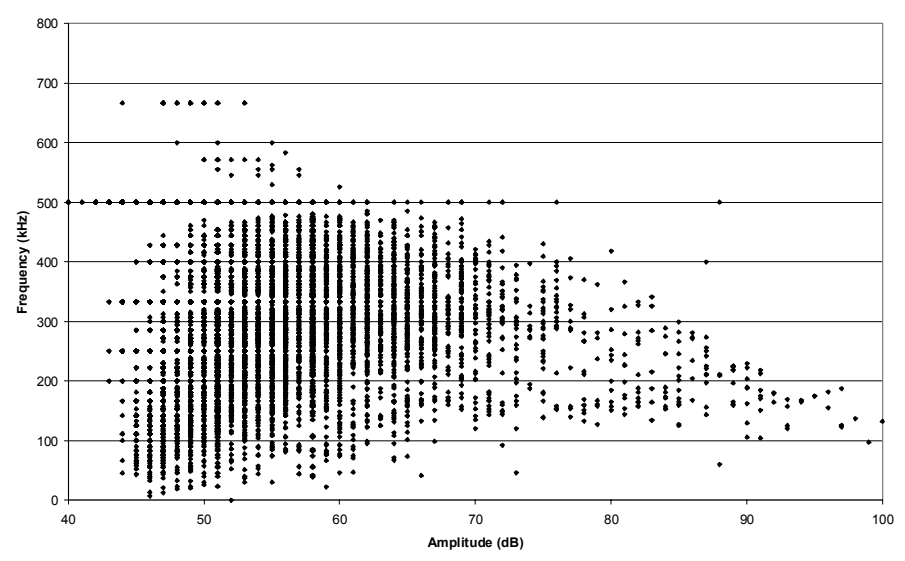

Test B9, ch 182: Frequency vs Amplitude (4th quarter)

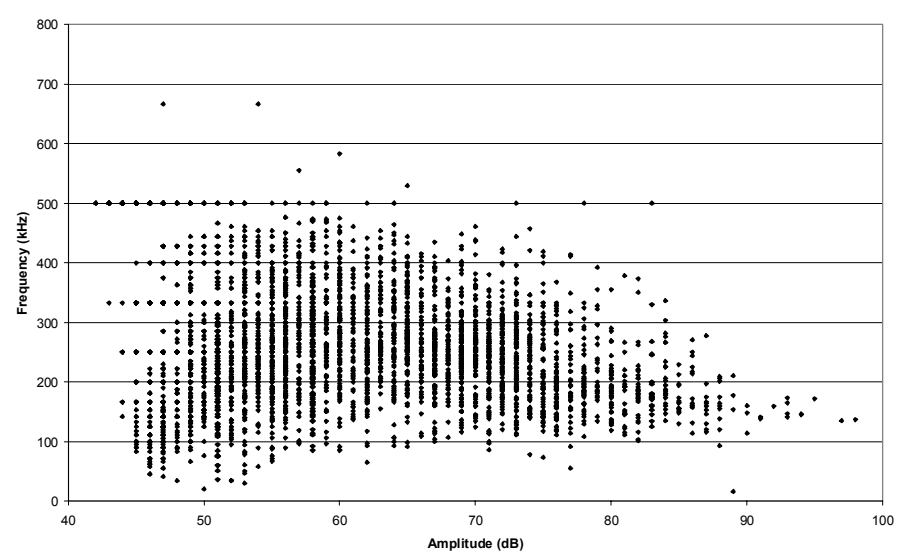

Appendix-D: Frequency vs. Amplitude Plots for Bending Specimens 

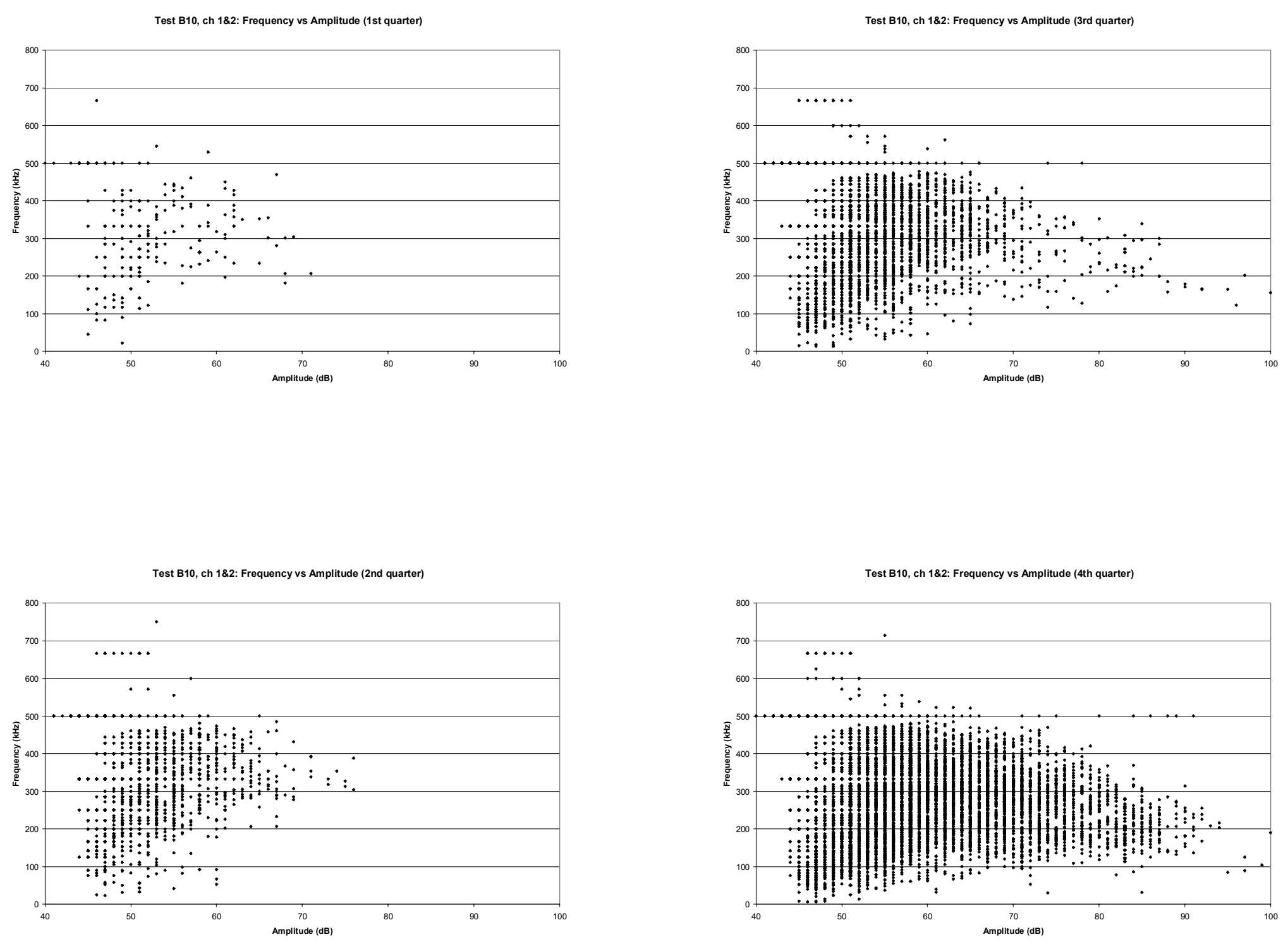

Appendix-D: Frequency vs. Amplitude Plots for Bending Specimens 
Test C1, ch 182: Frequency vs Amplitude (1st quarter)

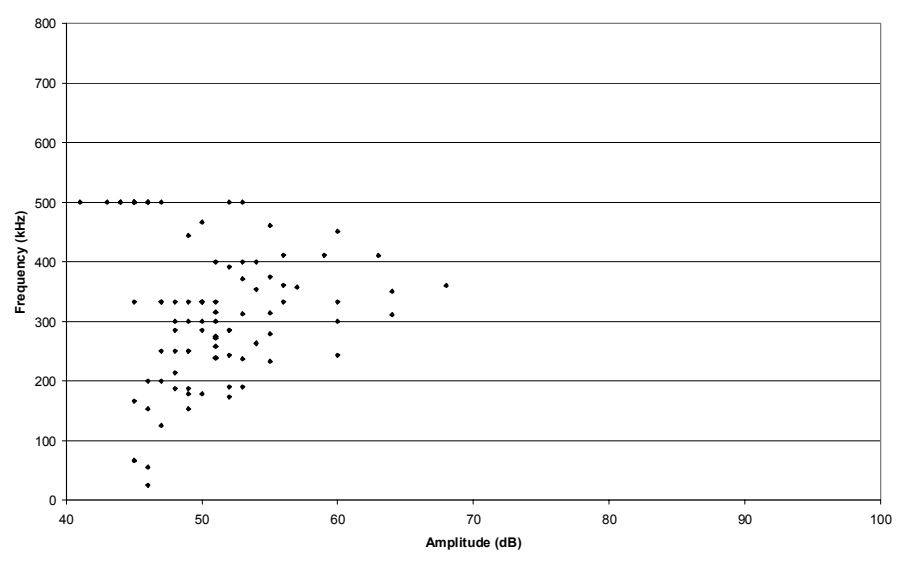

Test C1, ch 182: Frequency vs Amplitude (2nd quarter)

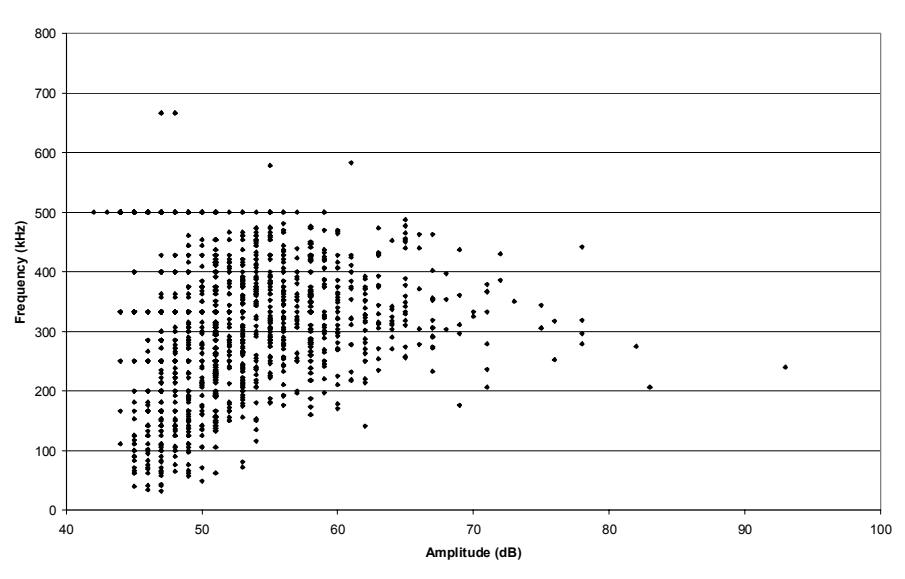

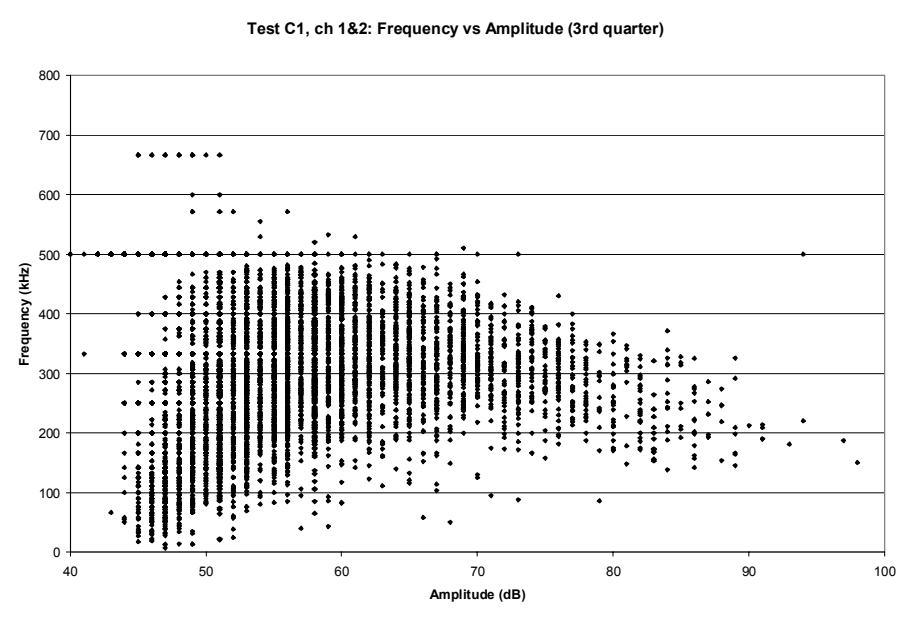

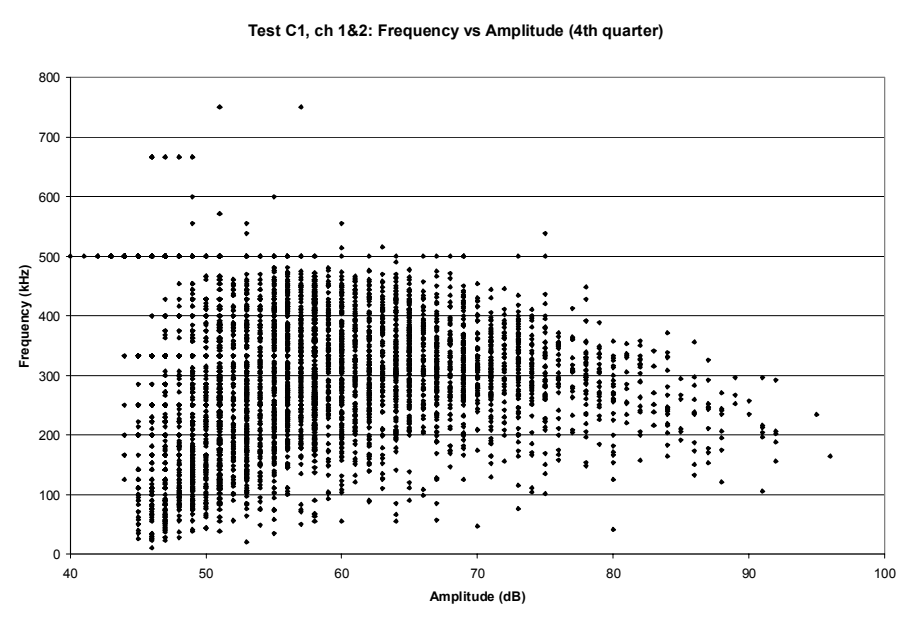

Appendix-D: Frequency vs. Amplitude Plots for Bending Specimens 

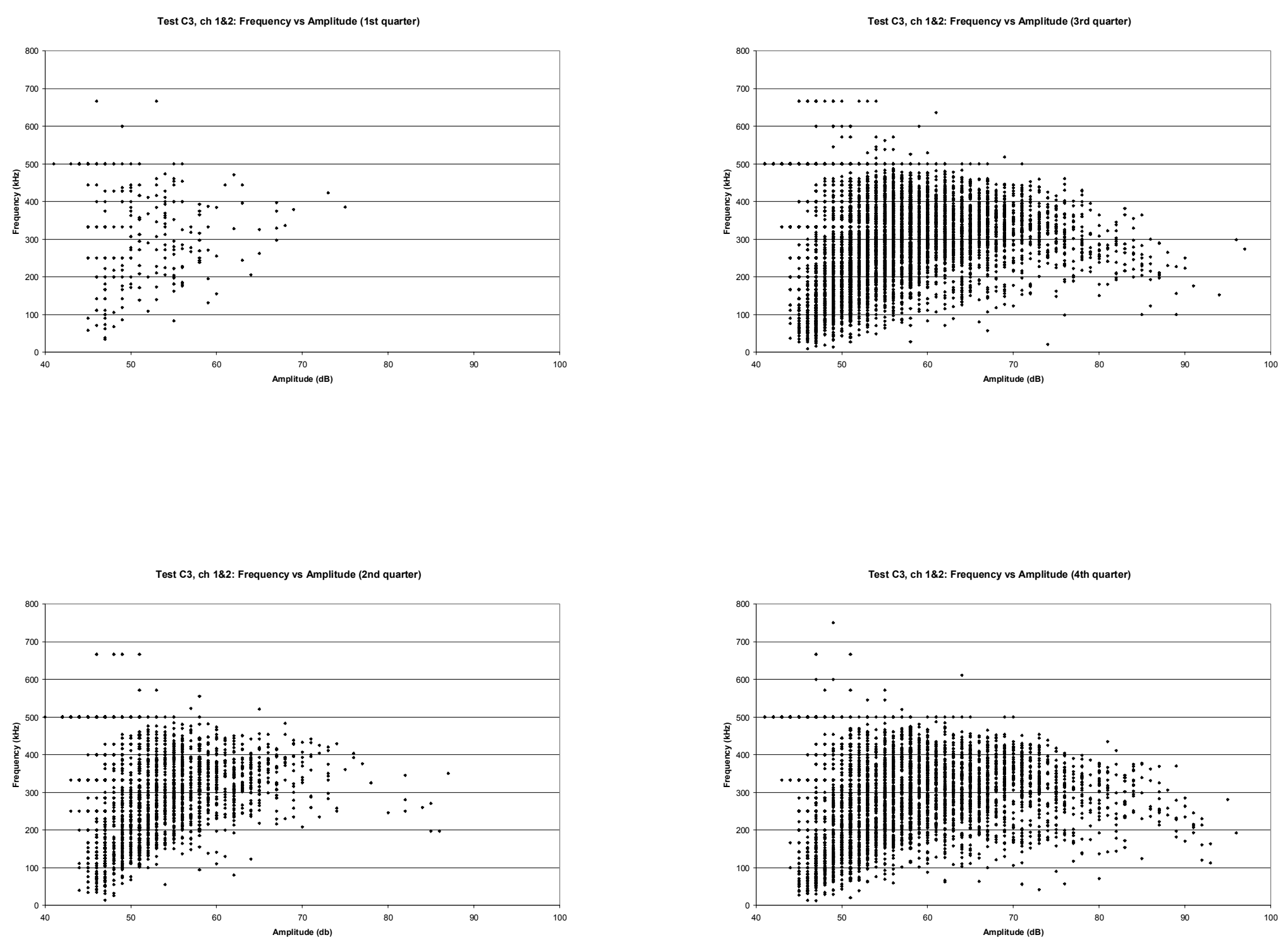

Appendix-D: Frequency vs. Amplitude Plots for Bending Specimens 

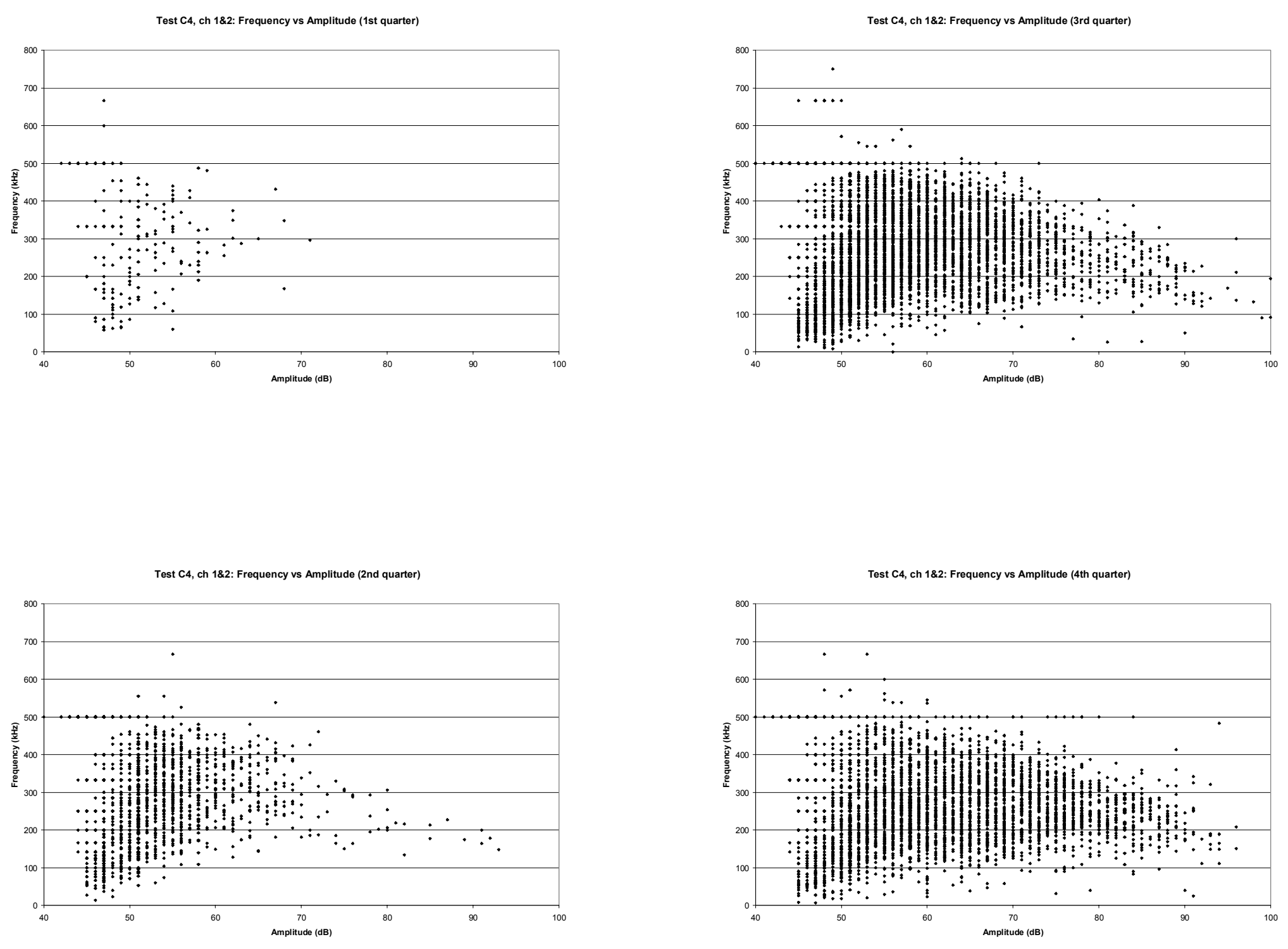

Appendix-D: Frequency vs. Amplitude Plots for Bending Specimens 

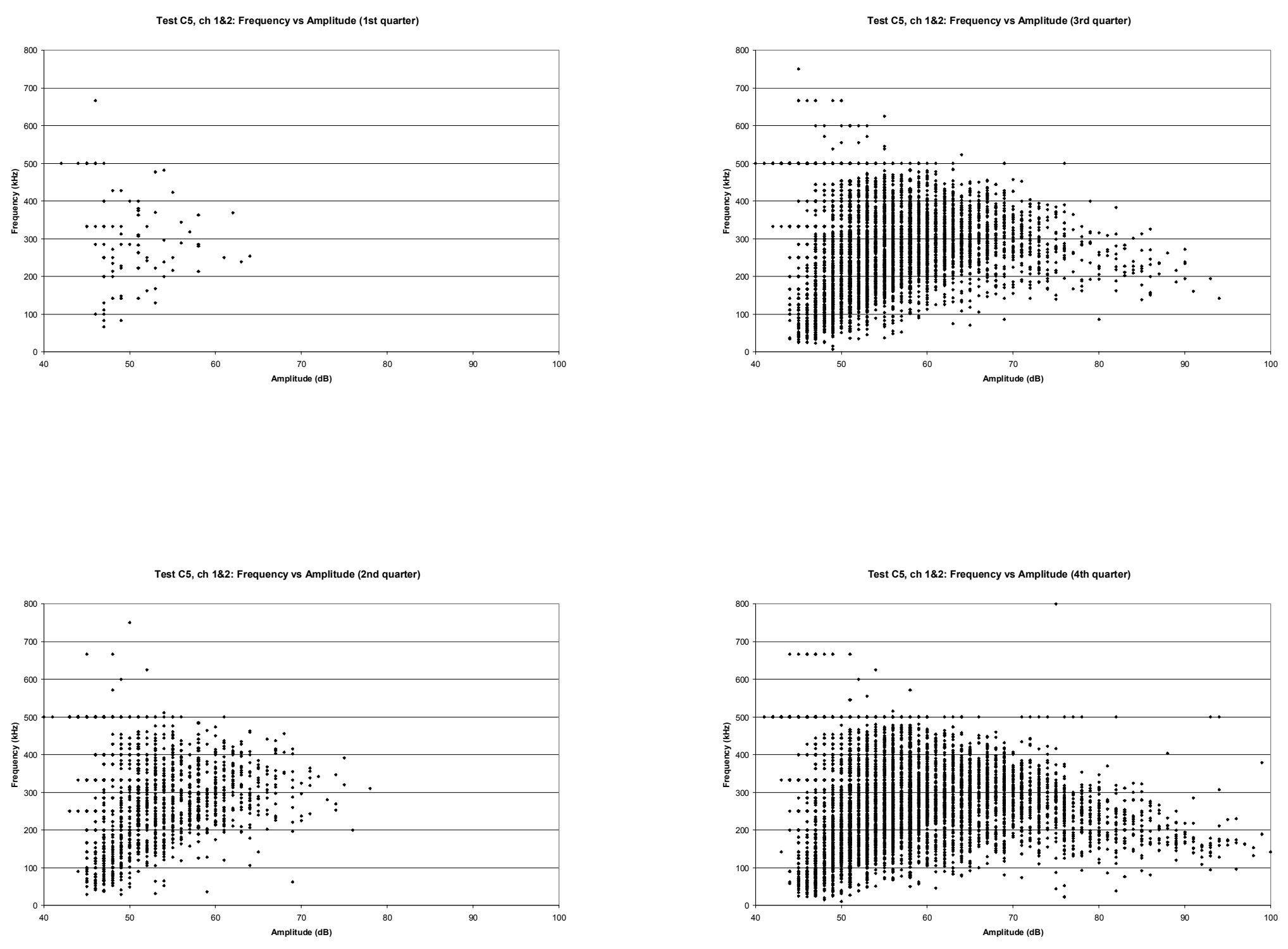

Appendix-D: Frequency vs. Amplitude Plots for Bending Specimens 


\section{Appendix-E}

Amplitude vs. Duration Plots

Bending Specimens

Tests B1, B3-B5, B6, B8-B10, C1, C3-C5

Appendix-E: Amplitude vs. Duration Plots for Bending Specimens 
Test B1, ch 182: Amplitude vs Duration (1st quarter)

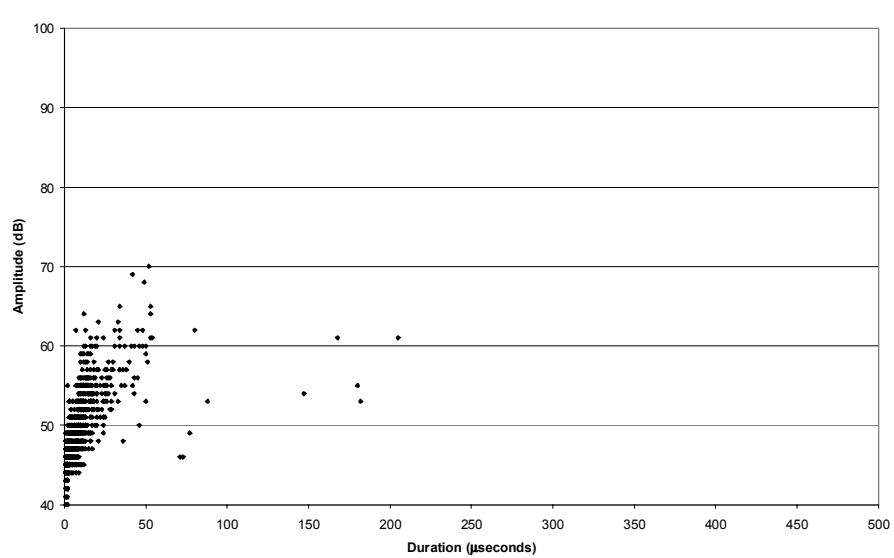

Test B1, ch 182: Amplitude vs Duration (2nd quarter)

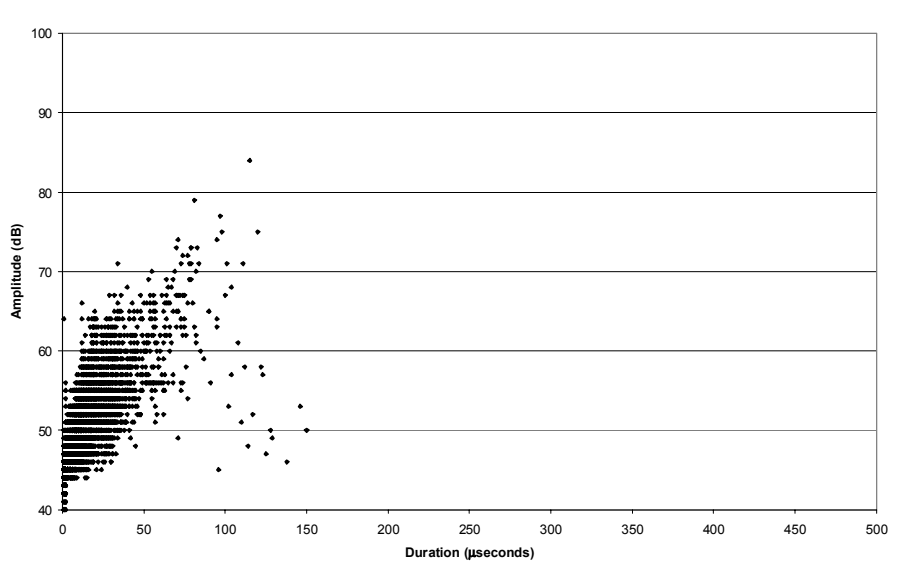

Test B1, ch 182: Amplitude vs Duration (3rd quarter truncated)

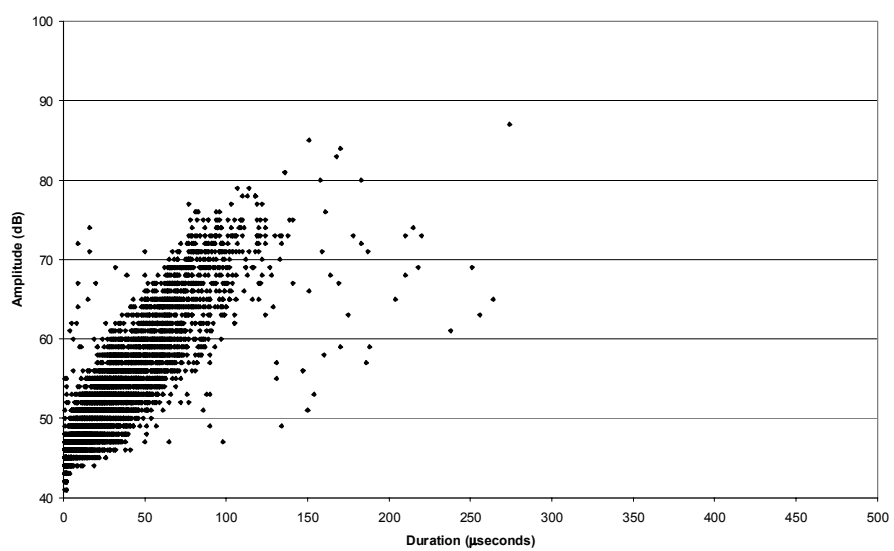

Appendix-E: Amplitude vs. Duration Plots for Bending Specimens 165 
Test B3, ch 182: Amplitude vs Duration (1st quarter)

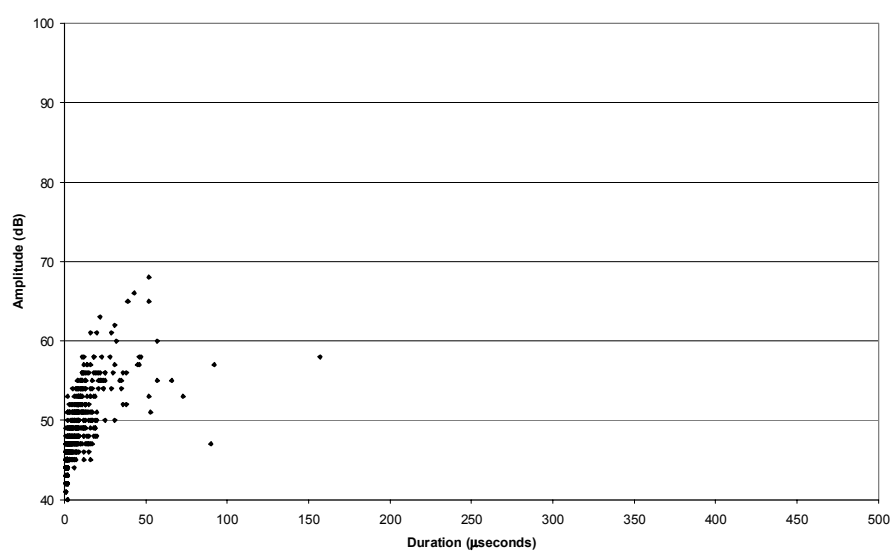

Test B3, ch 182: Amplitude vs Duration (2nd quarter)

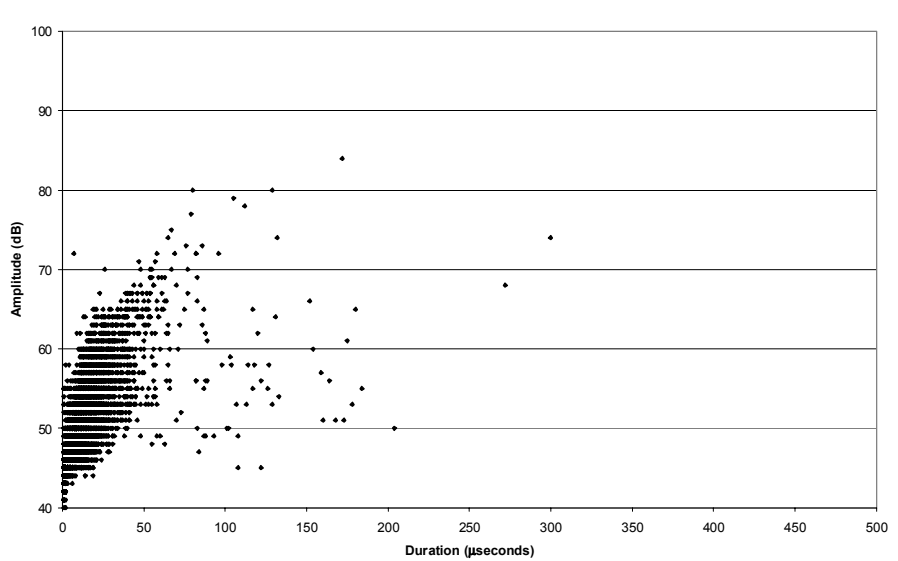

Test B3, ch 182: Amplitude vs Duration (3rd quarter)

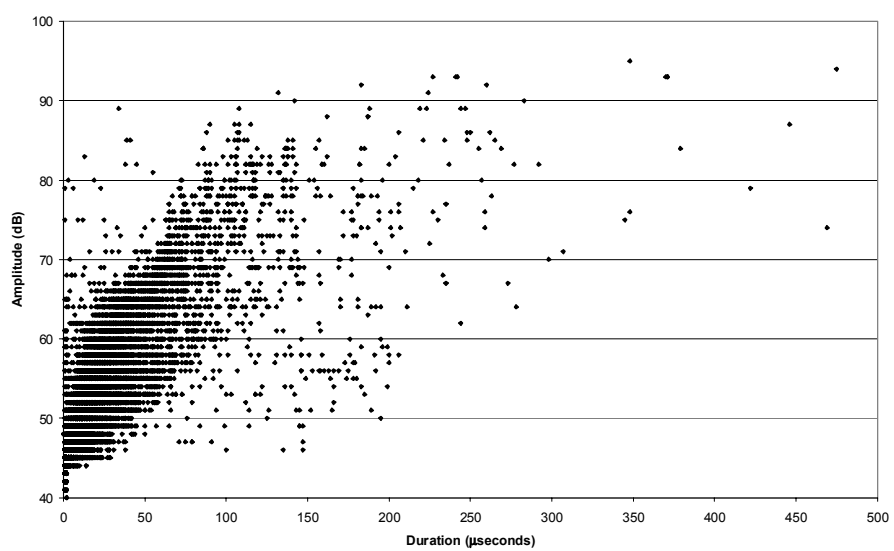

Test B3, ch 182: Amplitude vs Duration (4th quarter)

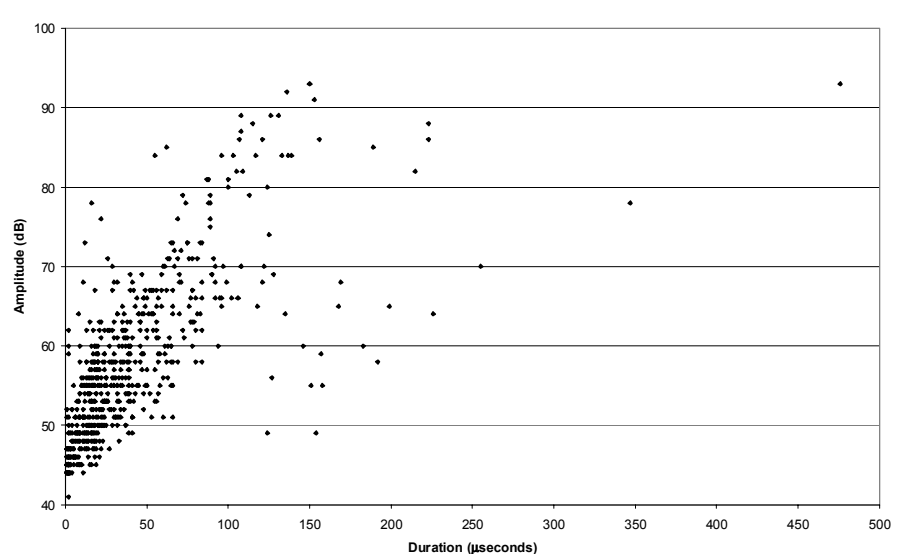

Appendix-E: Amplitude vs. Duration Plots for Bending Specimens 
Test B4, ch 182: Amplitude vs Duration (1st quarter)

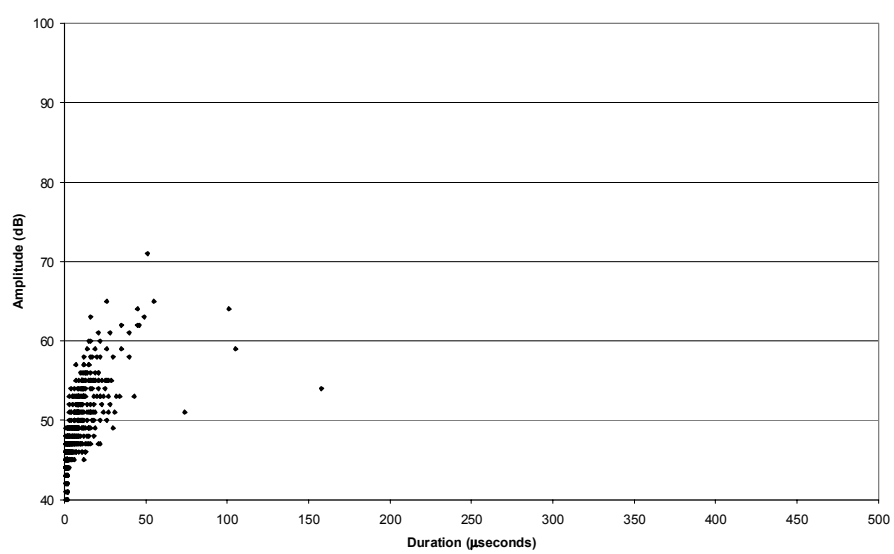

Test B4, ch 182: Amplitude vs Duration (2nd quarter)

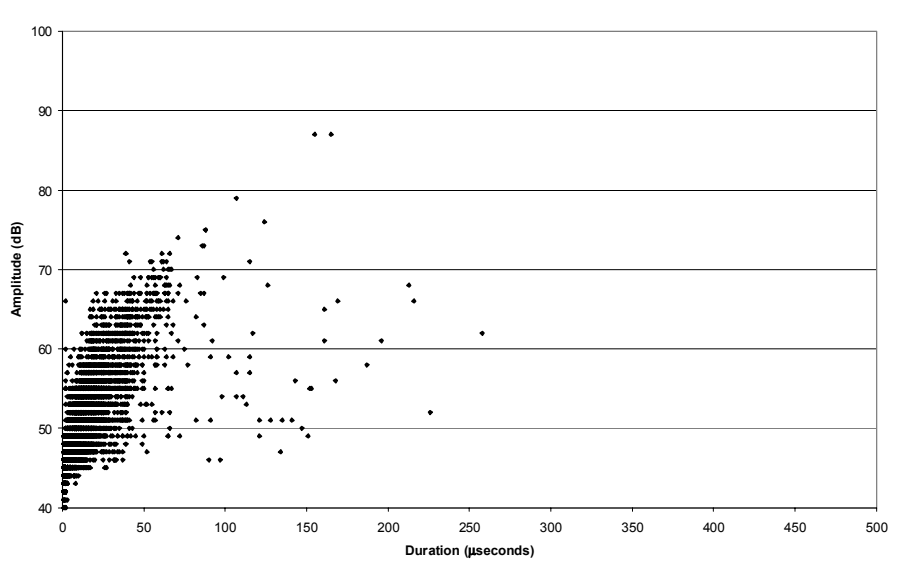

Test B4, ch 182: Amplitude vs Duration (3rd quarter)

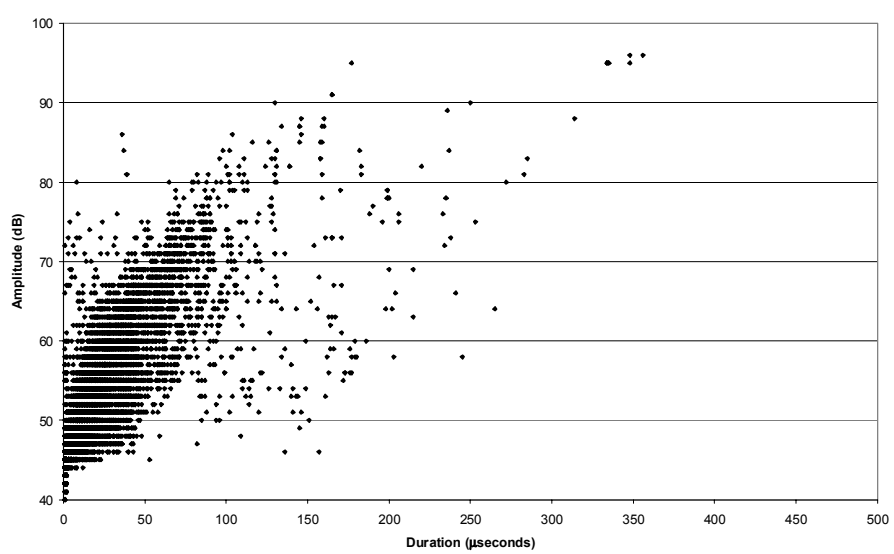

Test B4, ch 182: Amplitude vs Duration (4th quarter)

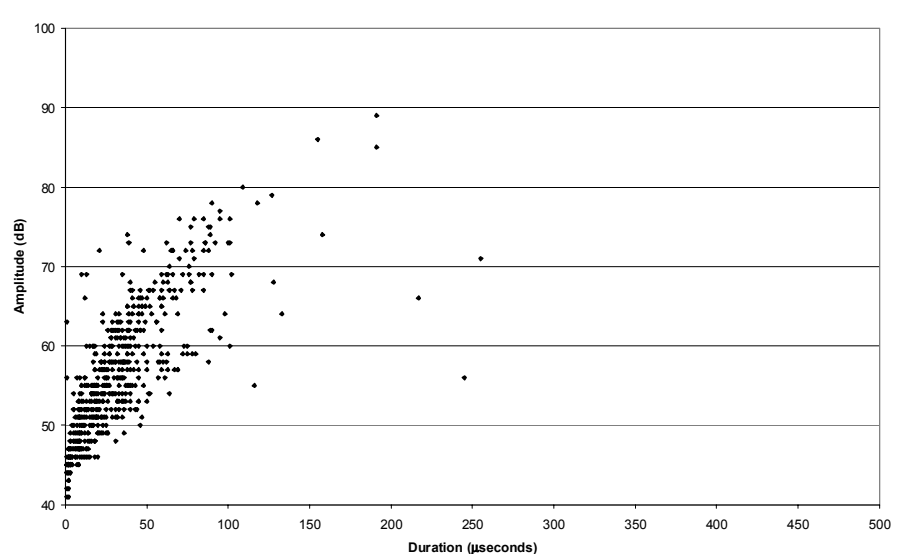

Appendix-E: Amplitude vs. Duration Plots for Bending Specimens 
Test B5, ch 182: Amplitude vs Duration (1st quarter)

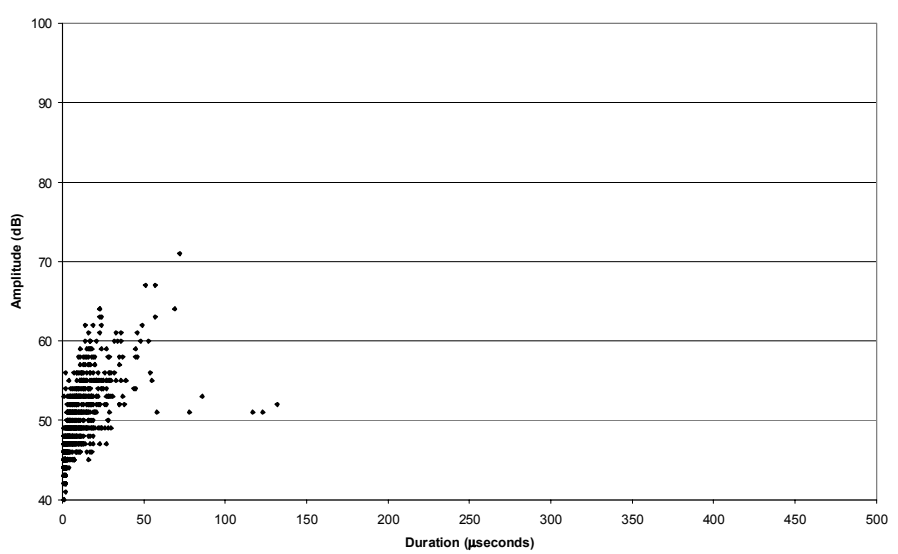

Test B5, ch 182: Amplitude vs Duration (2nd quarter)

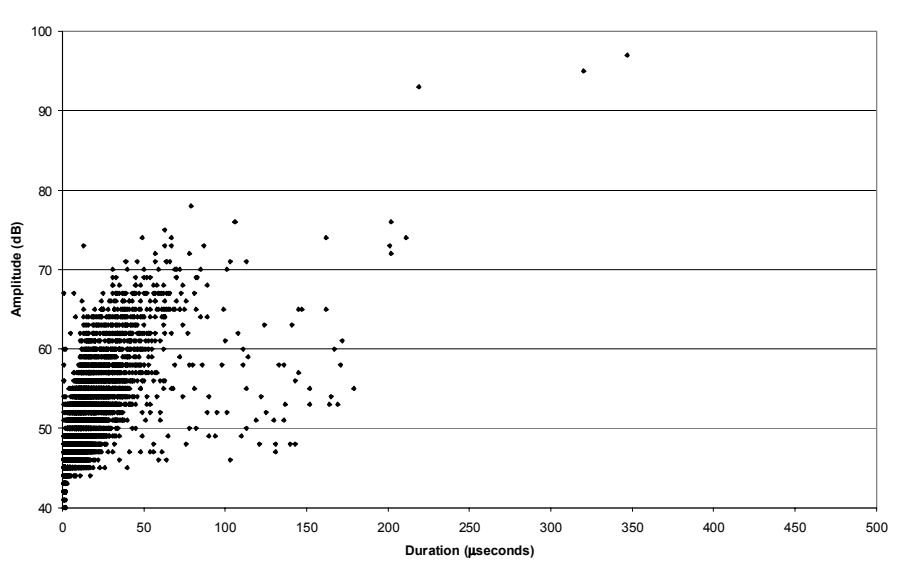

Test B5, ch 182: Amplitude vs Duration (3rd quarter)

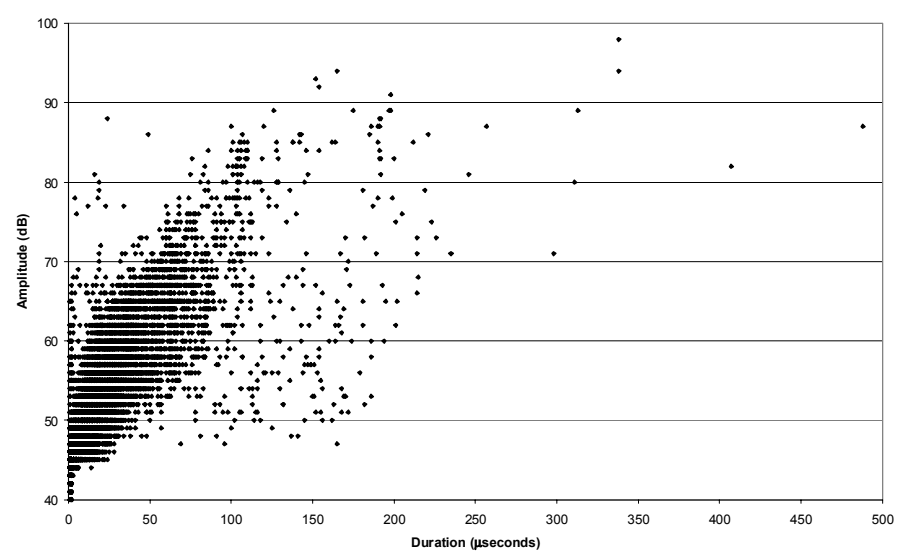

Test B5, ch 182: Amplitude vs Duration (4th quarter)

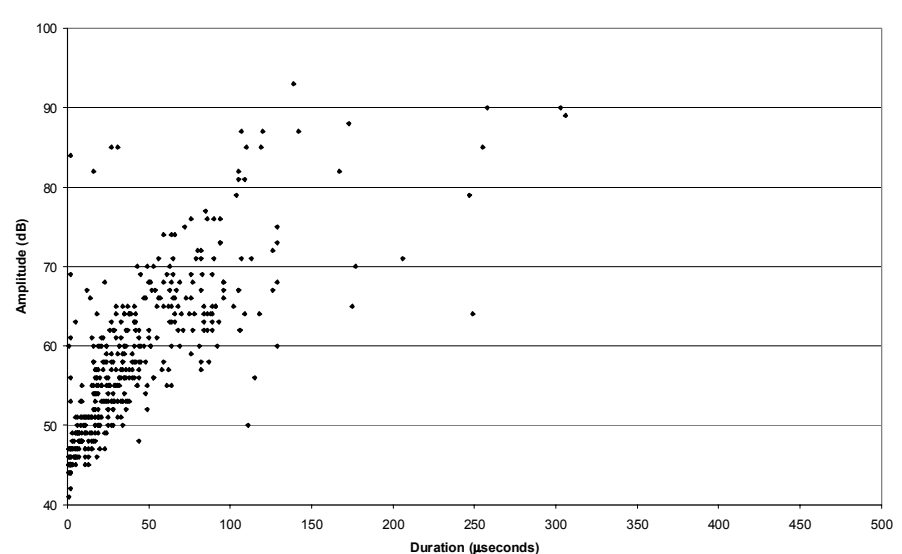

Appendix-E: Amplitude vs. Duration Plots for Bending Specimens 
Test B6, ch 182: Amplitude vs Duration (1st quarter)

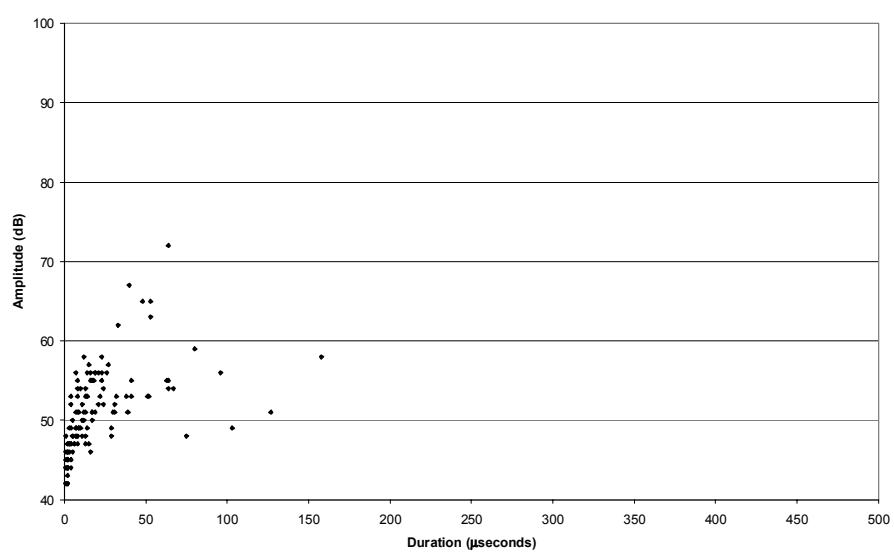

Test B6, ch 182: Amplitude vs Duration (2nd quarter)

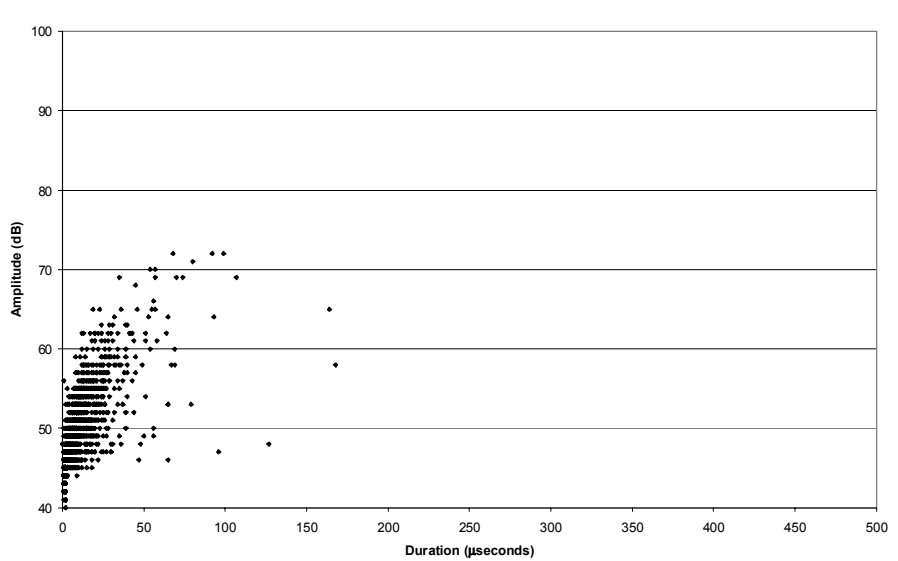

Test B6, ch 182: Amplitude vs Duration (3rd quarter)

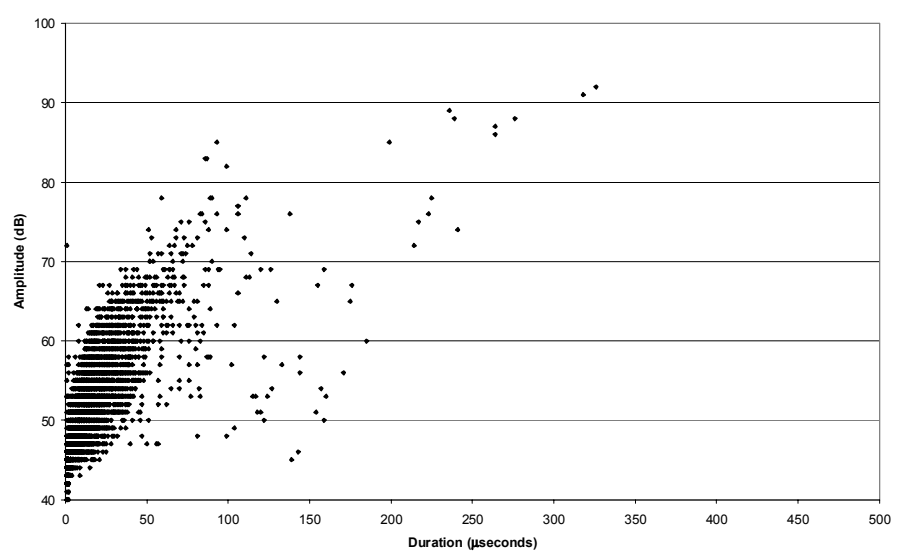

Test B6, ch 182: Amplitude vs Duration (4th quarter)

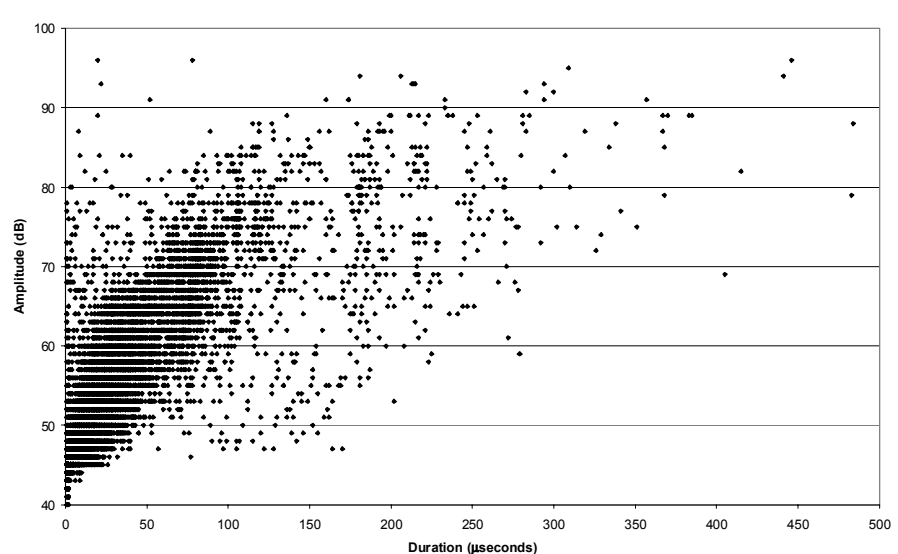

Appendix-E: Amplitude vs. Duration Plots for Bending Specimens 
Test B8, ch 182: Amplitude vs Duration (1st quarter)

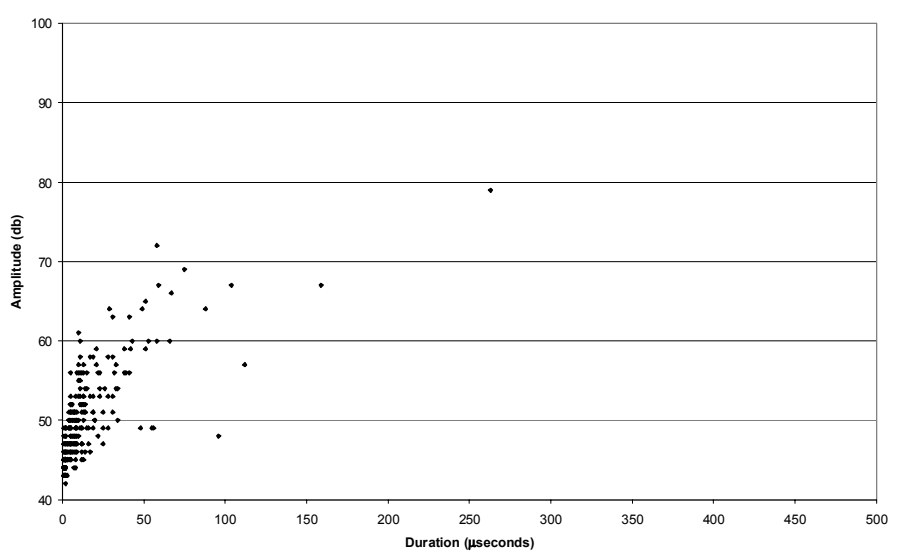

Test B8, ch 182: Amplitude vs Duration (2nd quarter)

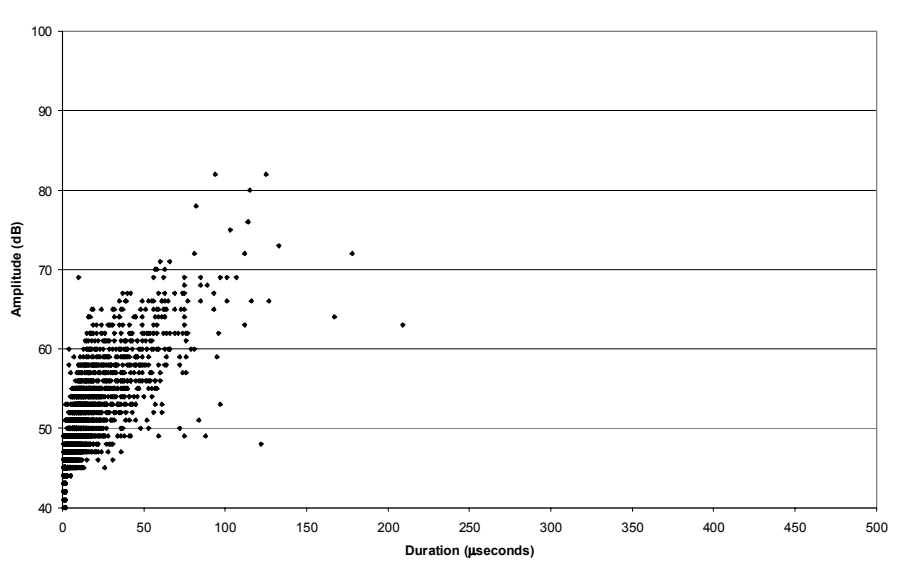

Test B8, ch 182: Amplitude vs Duration (3rd quarter)

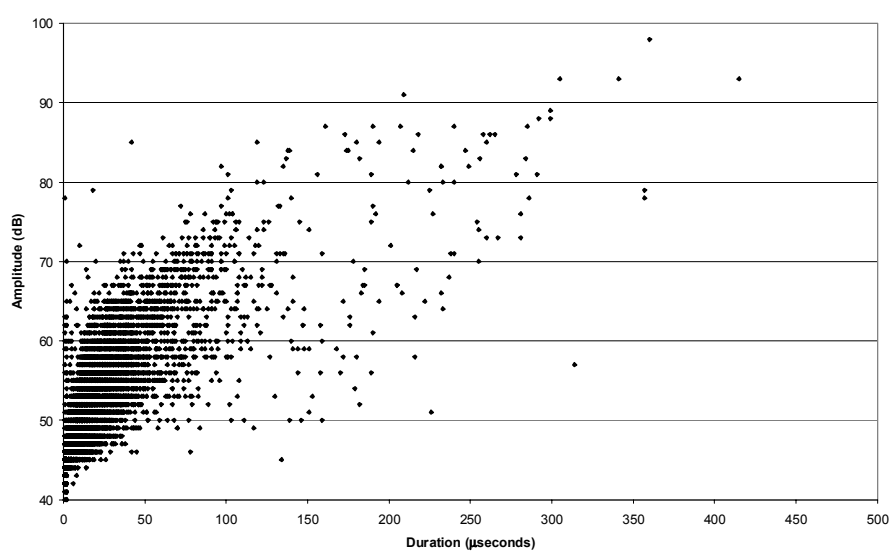

Test B8, ch 182: Amplitude vs Duration (4th quarter)

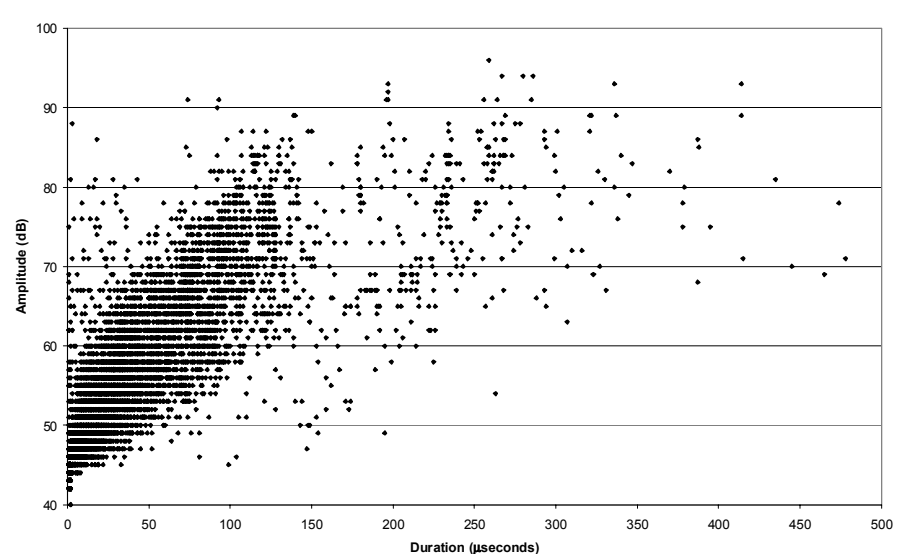

Appendix-E: Amplitude vs. Duration Plots for Bending Specimens 
Test B9, ch 122: Amplitude vs Duration (1st quarter)

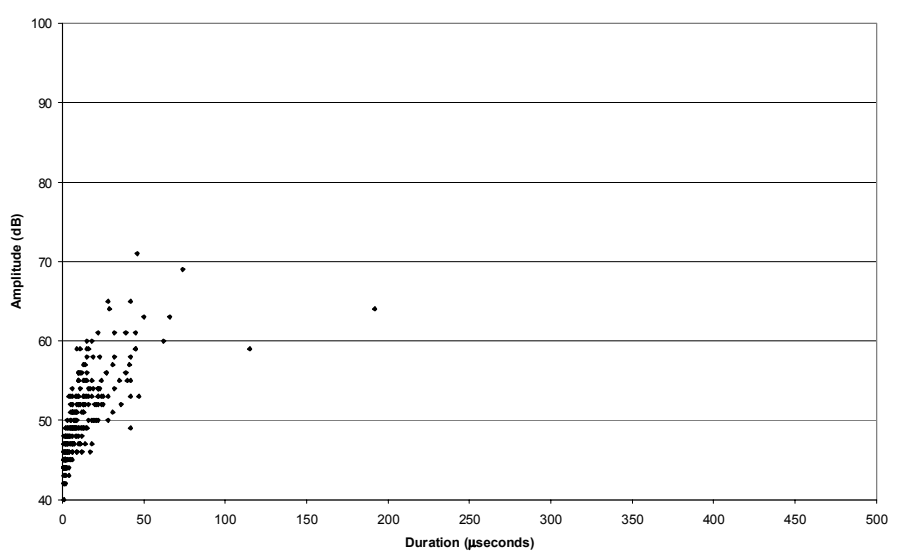

Test B9, ch 182: Amplitude vs Duration (2nd quarter)

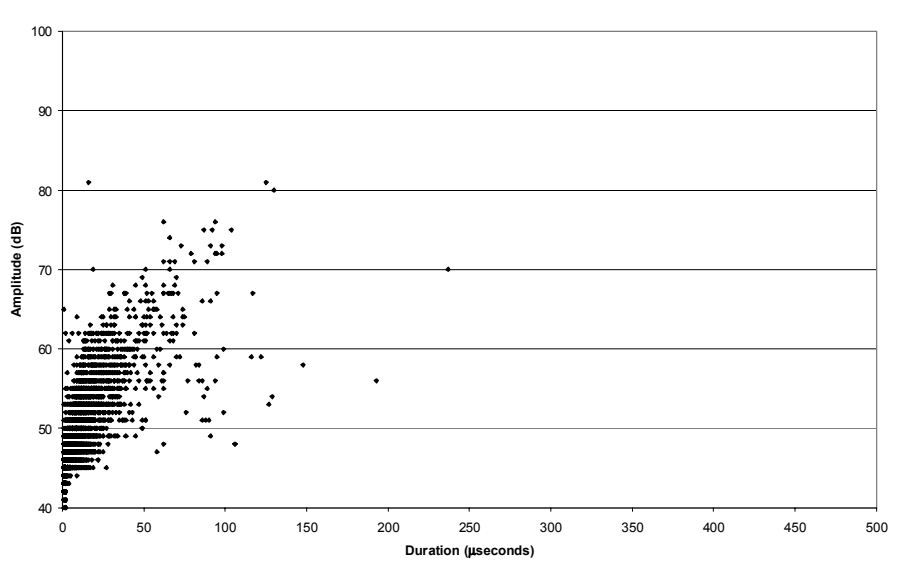

Test B9, ch 182: Amplitude vs Duration (3rd quarter)

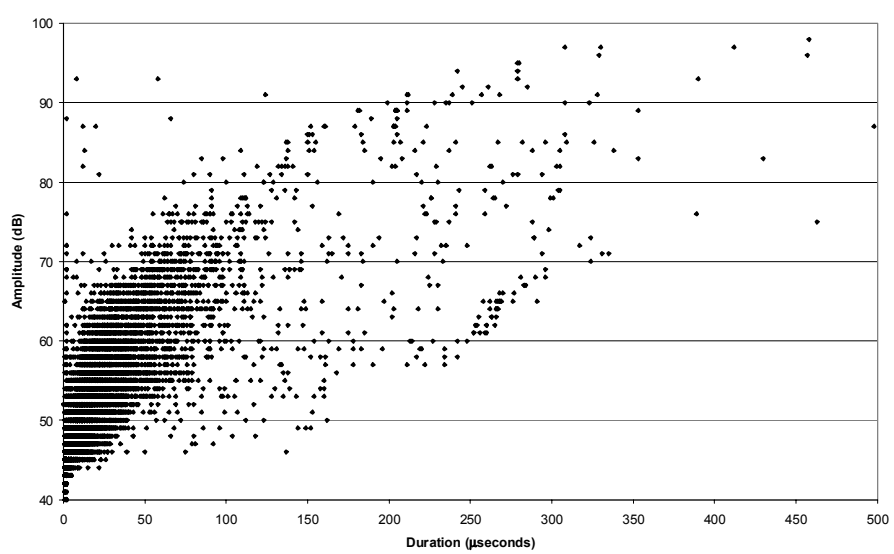

Test B9, ch 182: Amplitude vs Duration (4th quarter)

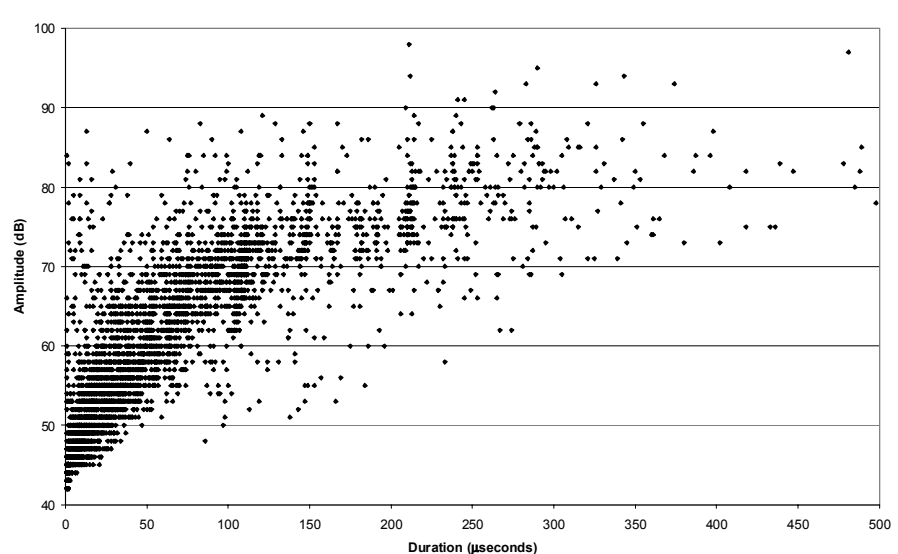

Appendix-E: Amplitude vs. Duration Plots for Bending Specimens 
Test B10, ch 182: Amplitude vs Duration (1st quarter)

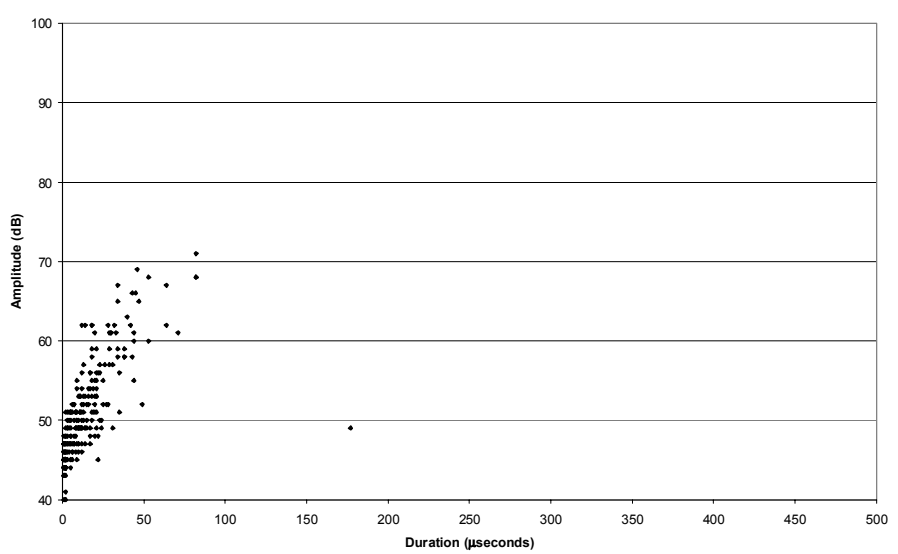

Test B10, ch 1\&2: Amplitude vs Duration (2nd quarter)

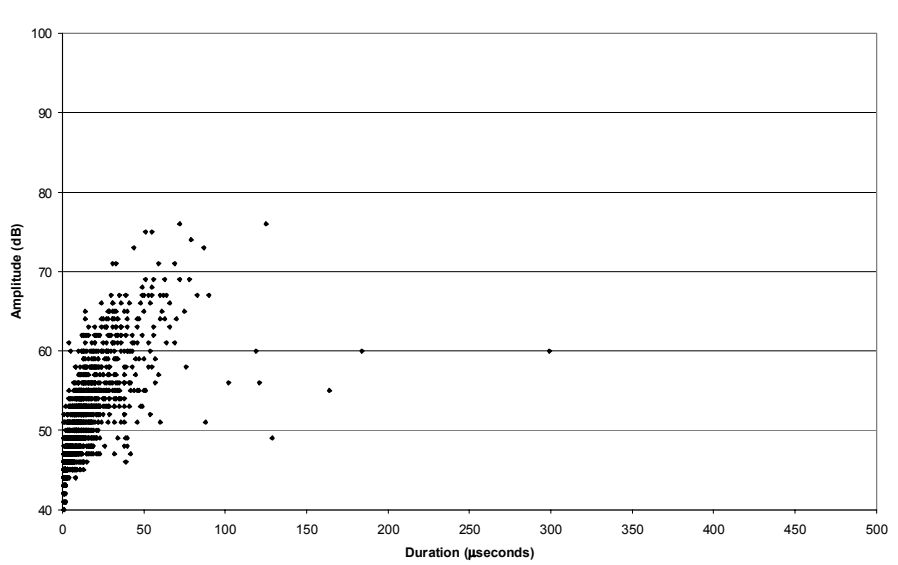

Test B10, ch 182: Amplitude vs Duration (3rd quarter)

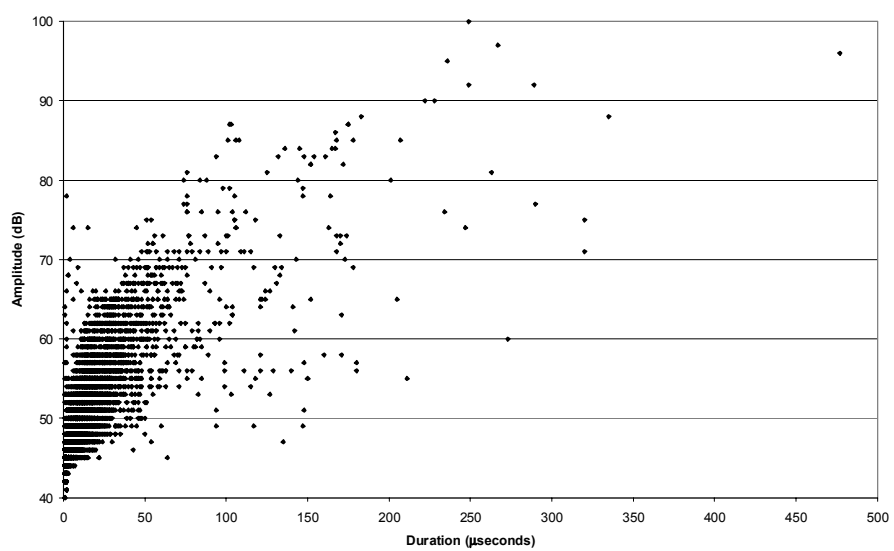

Test B10, ch 182: Amplitude vs Duration (4th quarter)

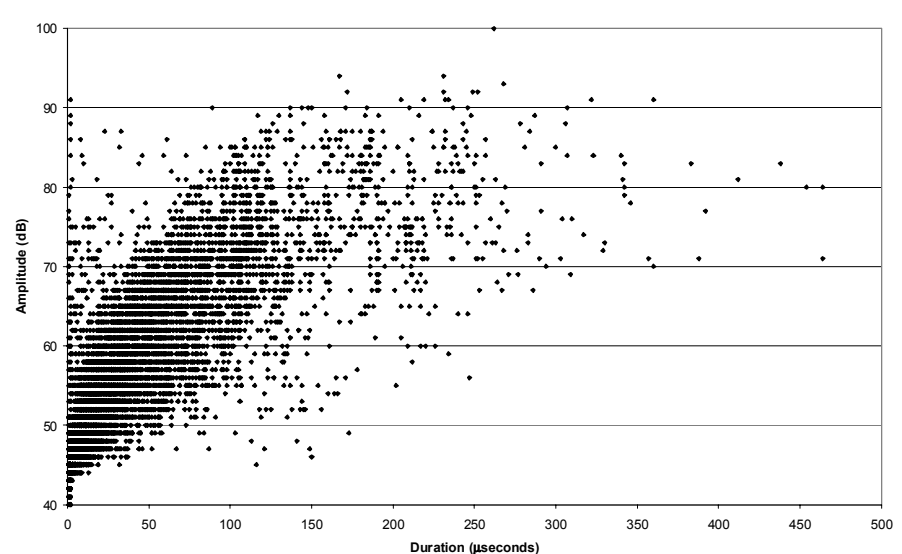

Appendix-E: Amplitude vs. Duration Plots for Bending Specimens 
Test C1, ch 182: Amplitude vs Duration (1st quarter)

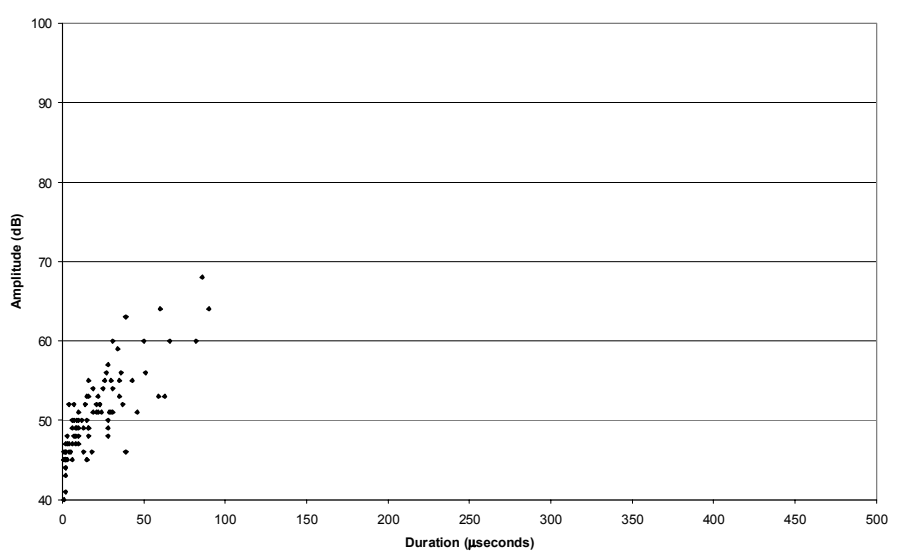

Test C1, ch 182: Amplitude vs Duration (2nd quarter)

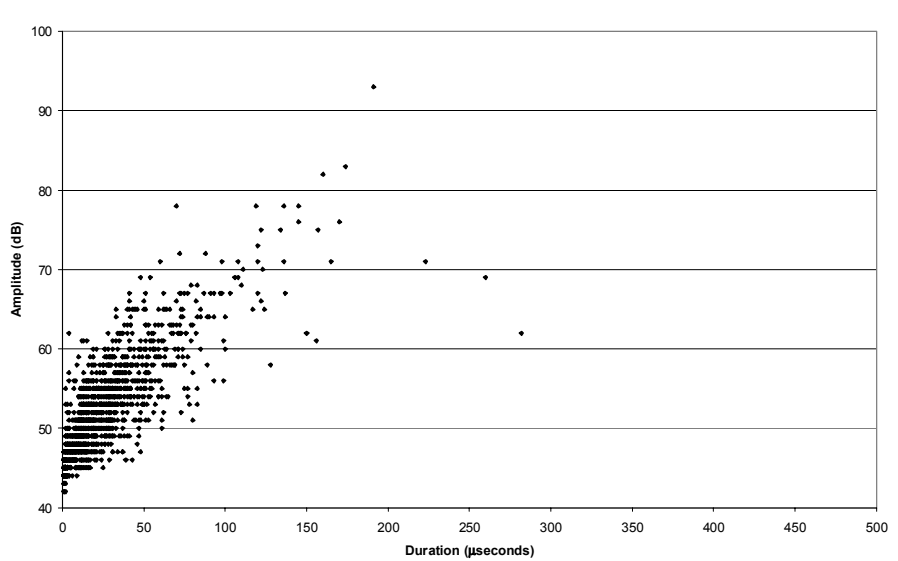

Test C1, ch 182: Amplitude vs Duration (3rd quarter)

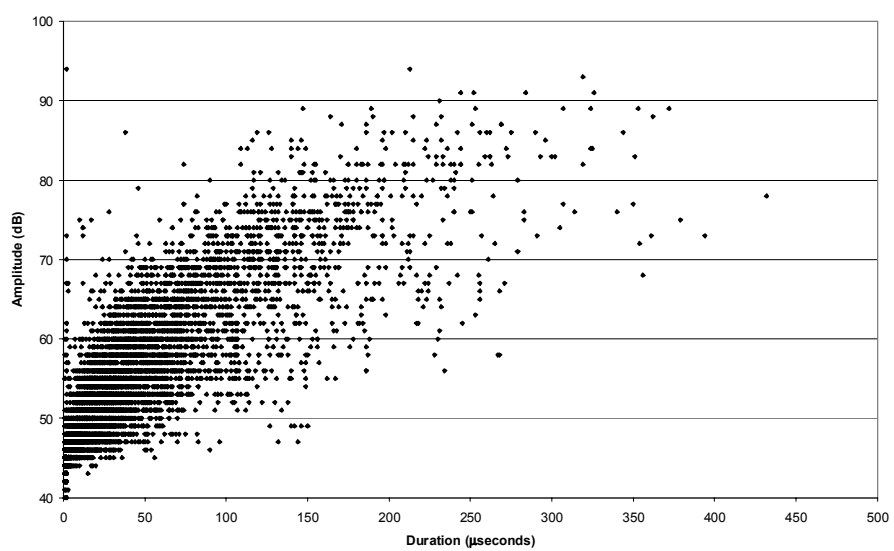

Test C1, ch 182: Amplitude vs Duration (4th quarter)

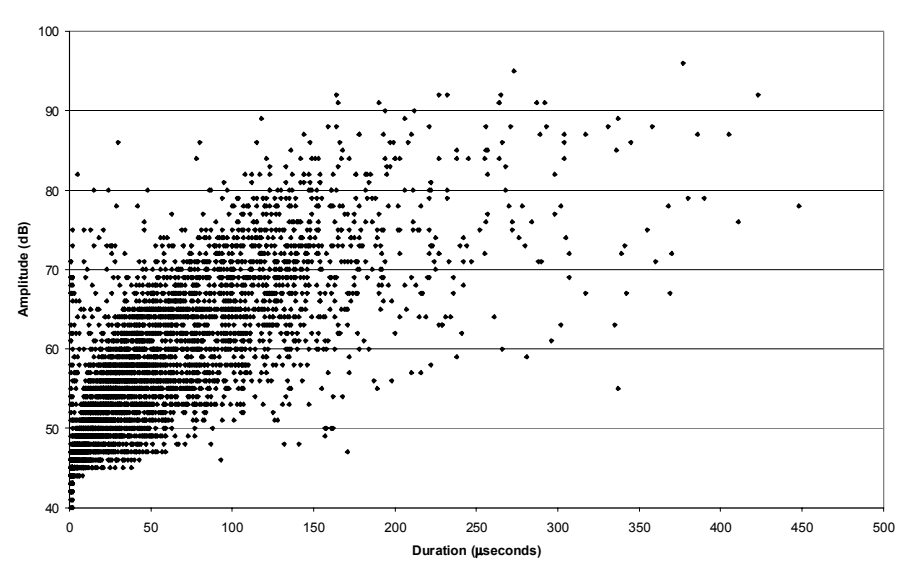

Appendix-E: Amplitude vs. Duration Plots for Bending Specimens 
Test C3, ch 182: Amplitude vs Duration (1st quarter)

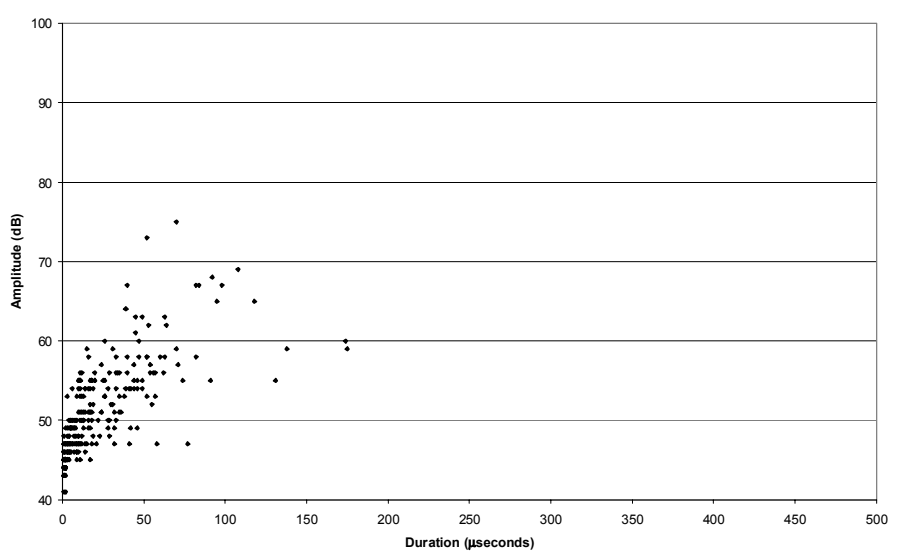

Test C3, ch 182: Amplitude vs Duration (2nd quarter)

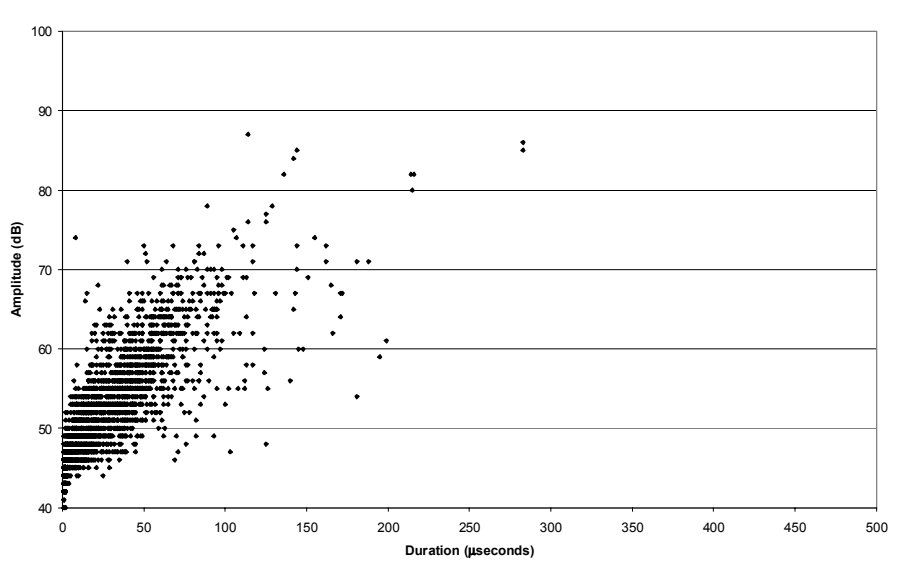

Test C3, ch 182: Amplitude vs Duration (3rd quarter)

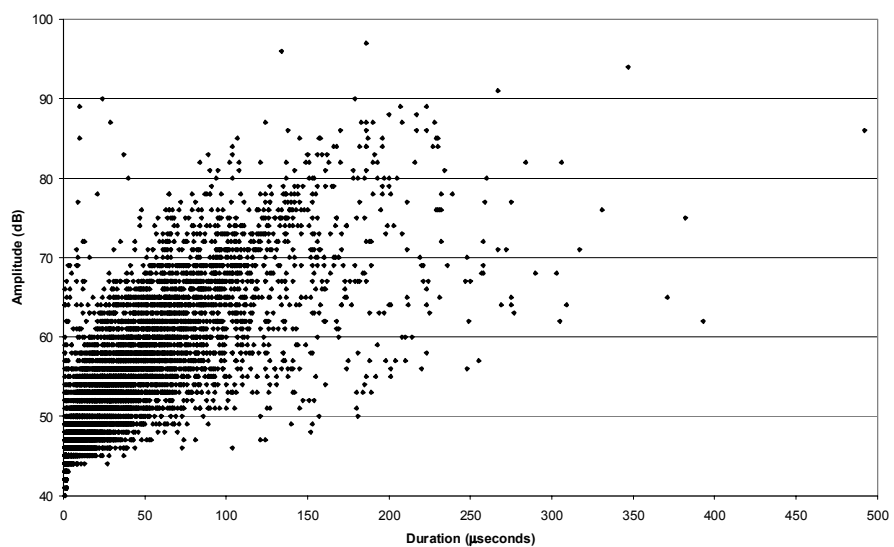

Test C3, ch 182: Amplitude vs Duration (4th quarter)

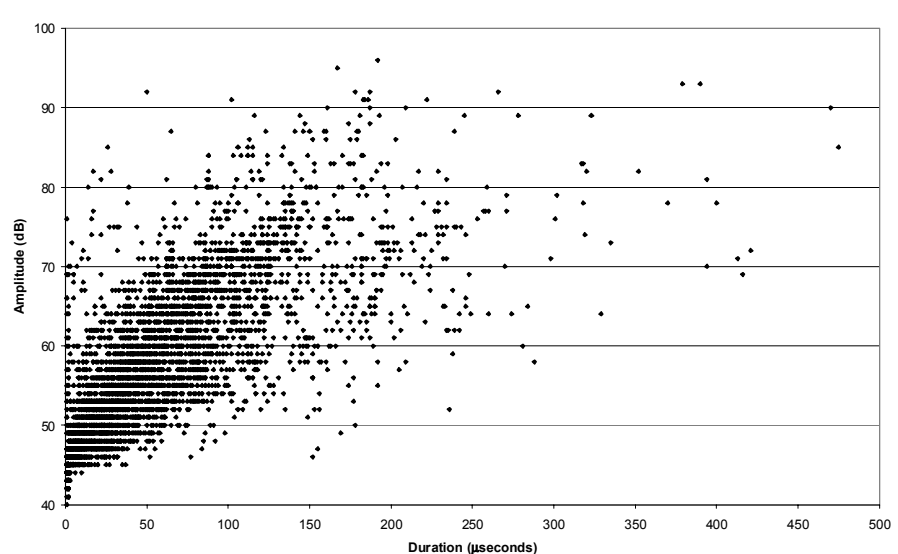

Appendix-E: Amplitude vs. Duration Plots for Bending Specimens 
Test C4, ch 182: Amplitude vs Duration (1st quarter)

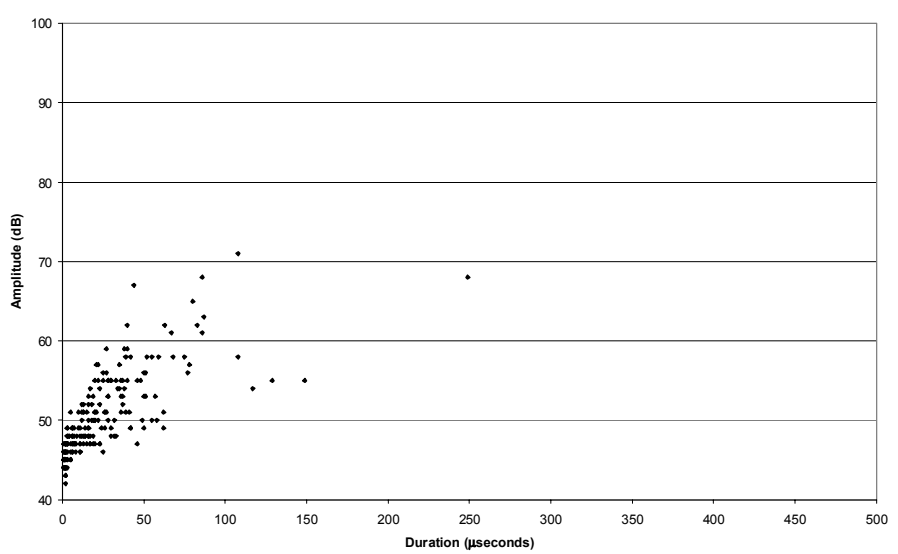

Test C4, ch 182: Amplitude vs Duration (2nd quarter)

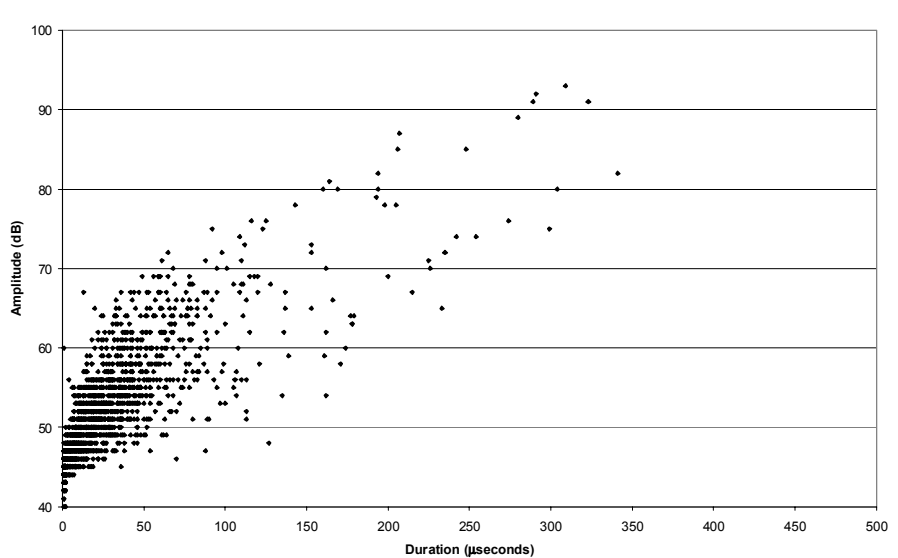

Test C4, ch 182: Amplitude ws Duration (3rd quarte)

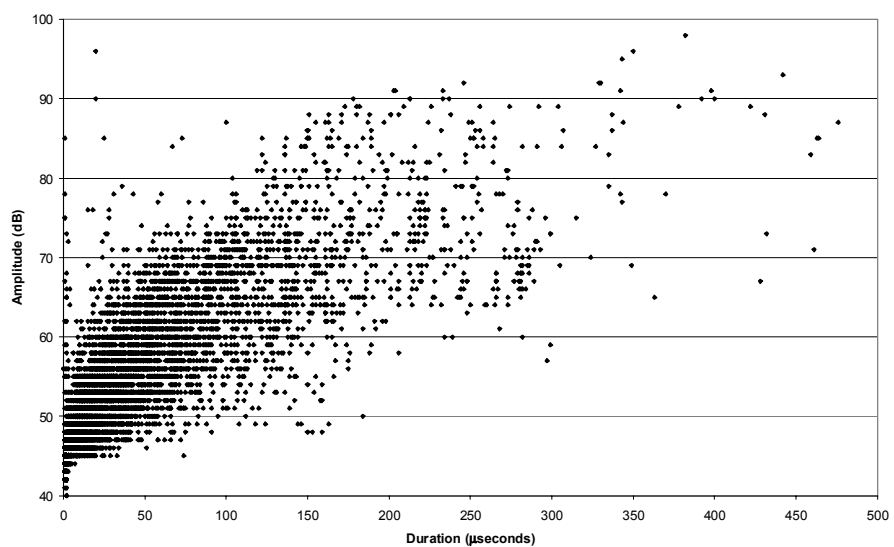

Test C4, ch 182: Amplitude vs Duration (4th quarter)

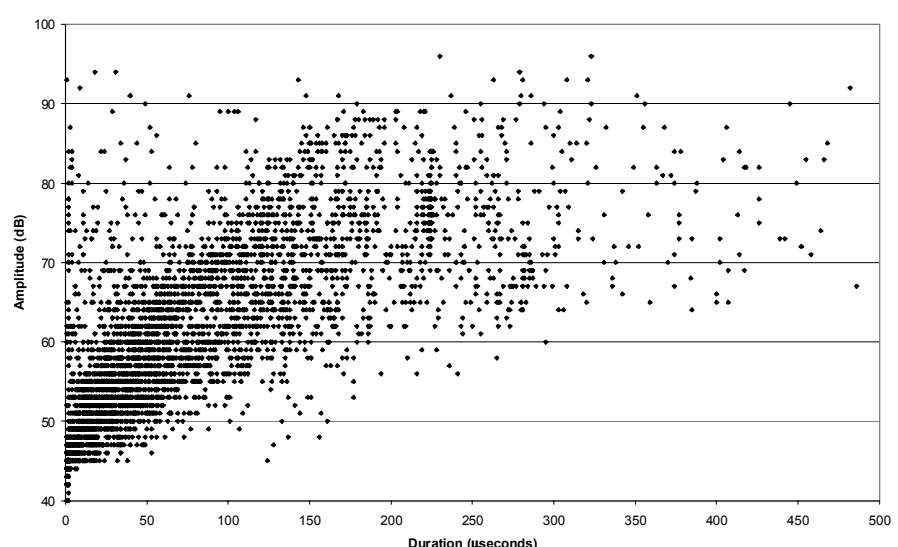

Appendix-E: Amplitude vs. Duration Plots for Bending Specimens 
Test C5, ch 182: Amplitude vs Duration (1st quarter)

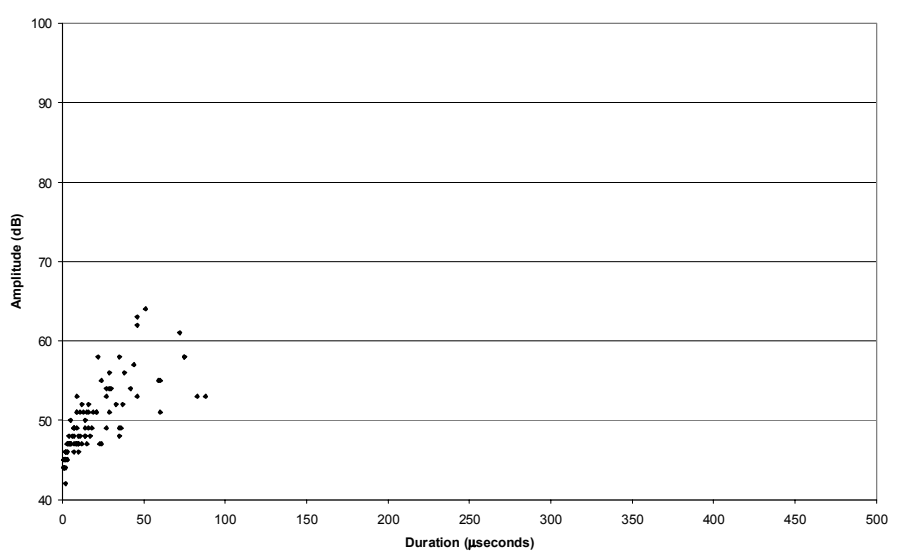

Test C5, ch 182: Amplitude vs Duration (2nd quarter)

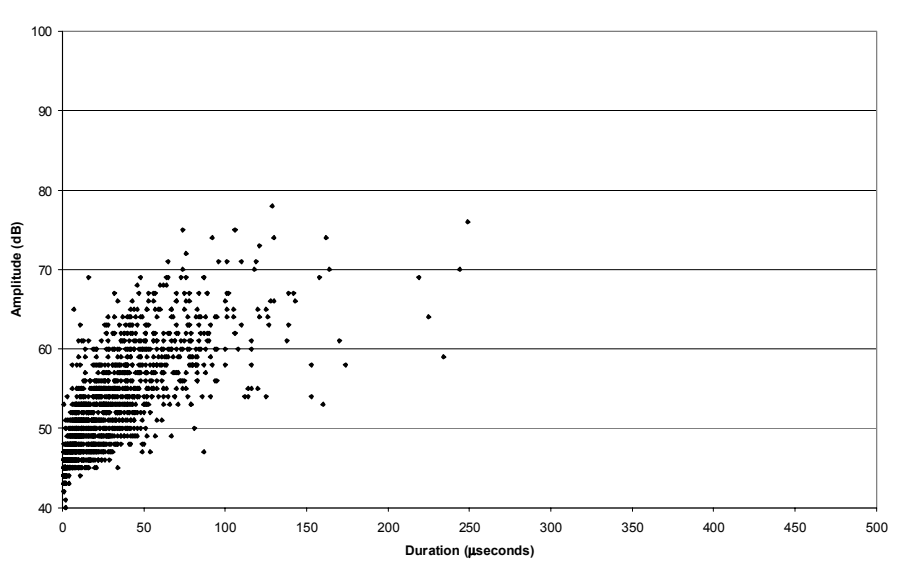

Test C5, ch 182: Amplitude vs Duration (3rd quarter)

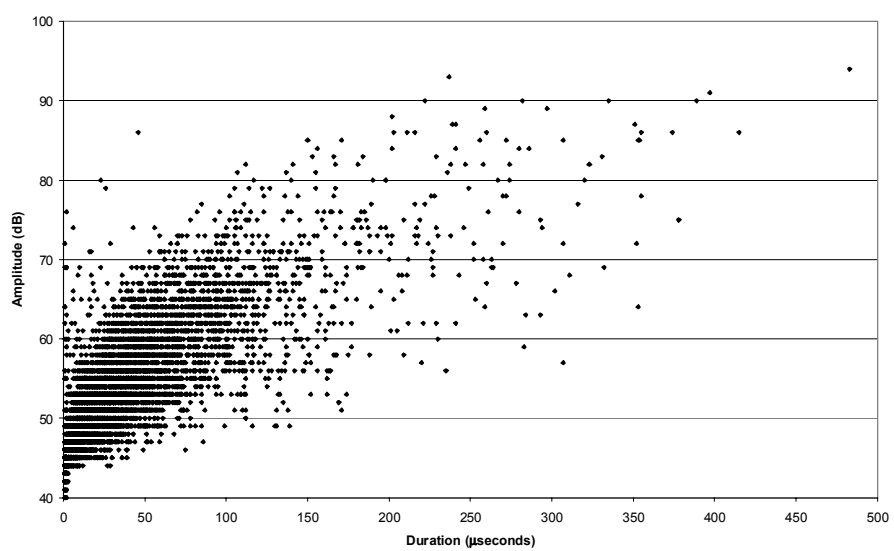

Test C5, ch 182: Amplitude vs Duration (4th quarter)

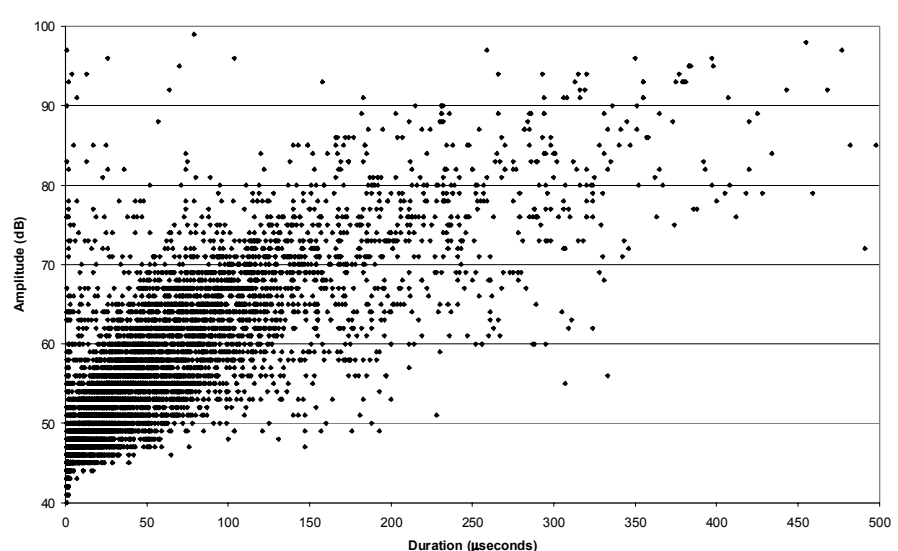

Appendix-E: Amplitude vs. Duration Plots for Bending Specimens 


\section{Appendix-F}

\section{¿Energy vs. Load Plots}

Bending Specimens

Tests B1, B3-B5, B6, B8-B10, C1, C3-C5

Appendix-F: $\Sigma$ Energy vs. Load Plots for Bending Specimens 
Test B1, ch 182: Eenergy vs Load (truncated)

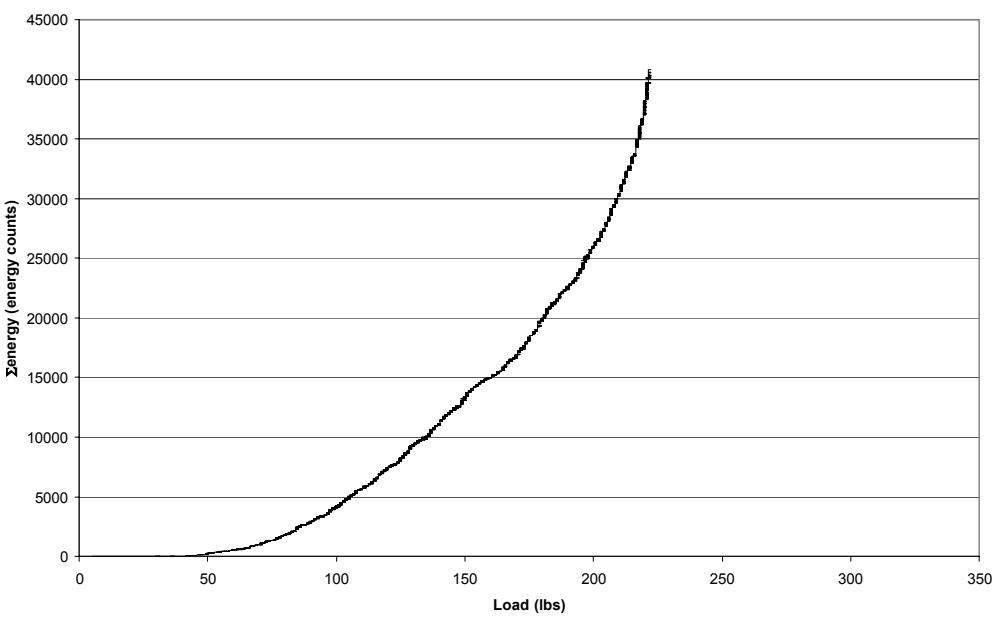

Test B3, ch 1\&2: Lenergy vs Load

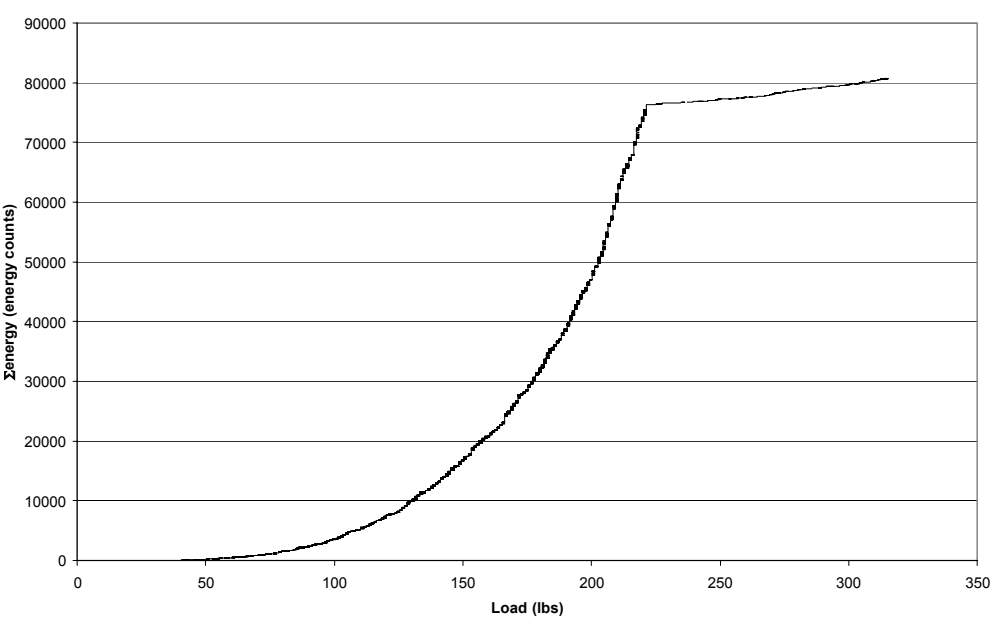

Test B4, ch 1\&2: Eenergy vs Load

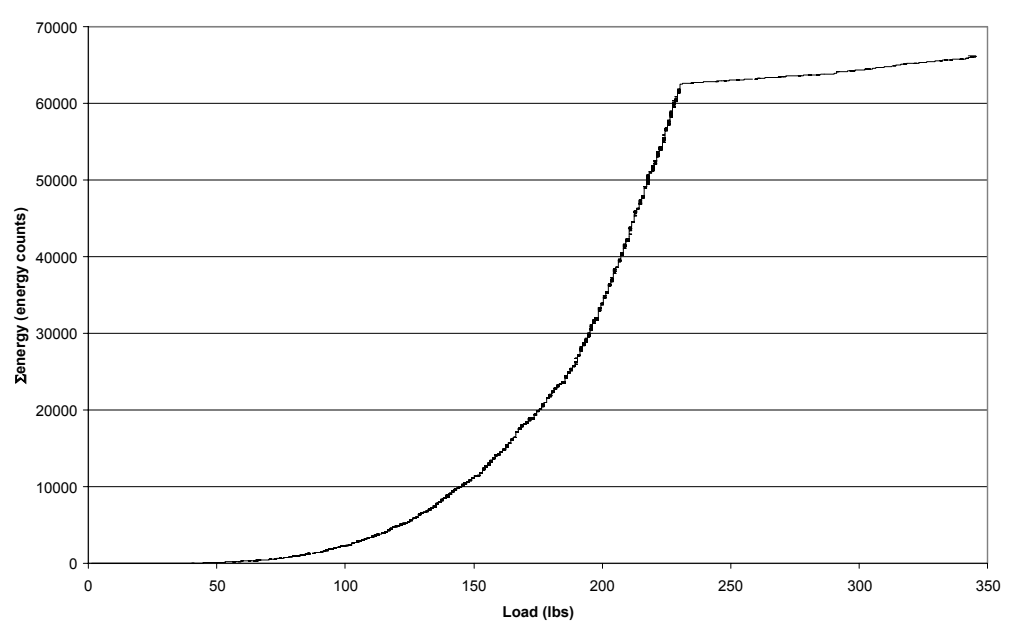

Test B5: Lenergy vs. Load

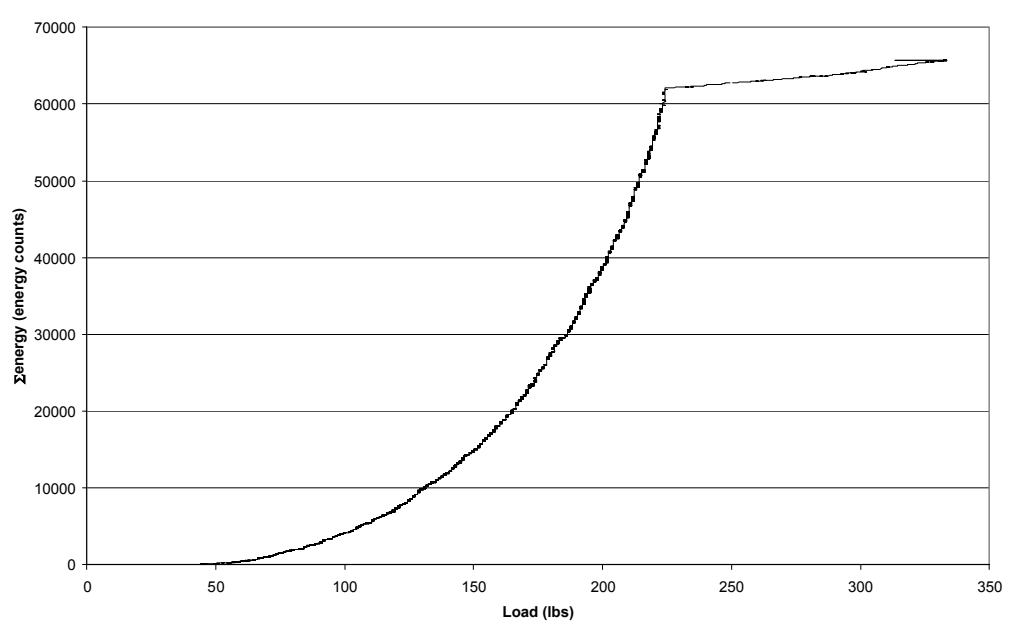

Appendix-F: $\Sigma$ Energy vs. Load Plots for Bending Specimens 
Test B6, ch 1\&2: Eenergy vs Load

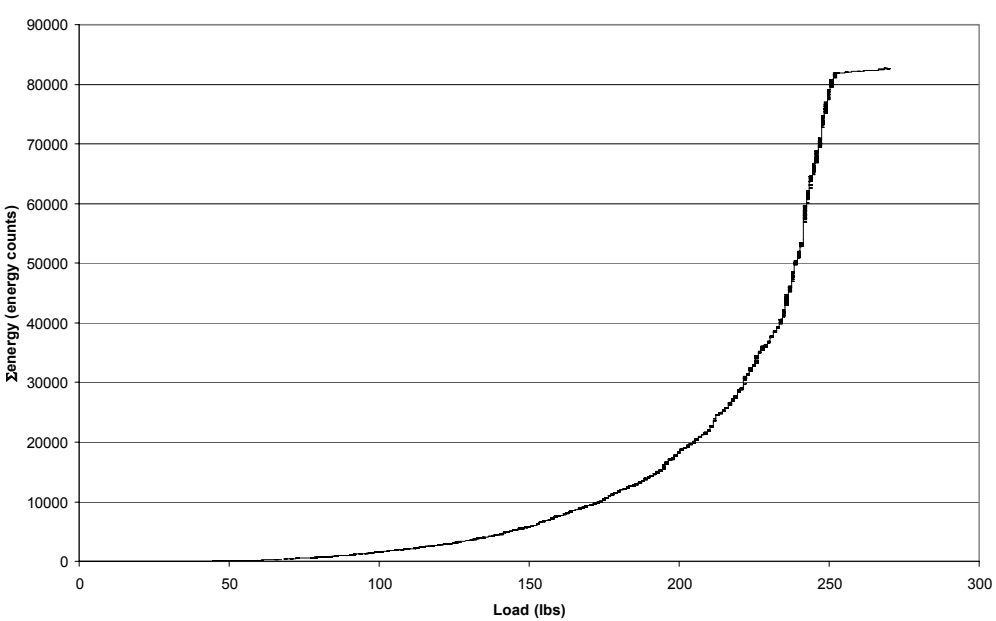

Test B8, ch 1\&2: Lenergy vs Load

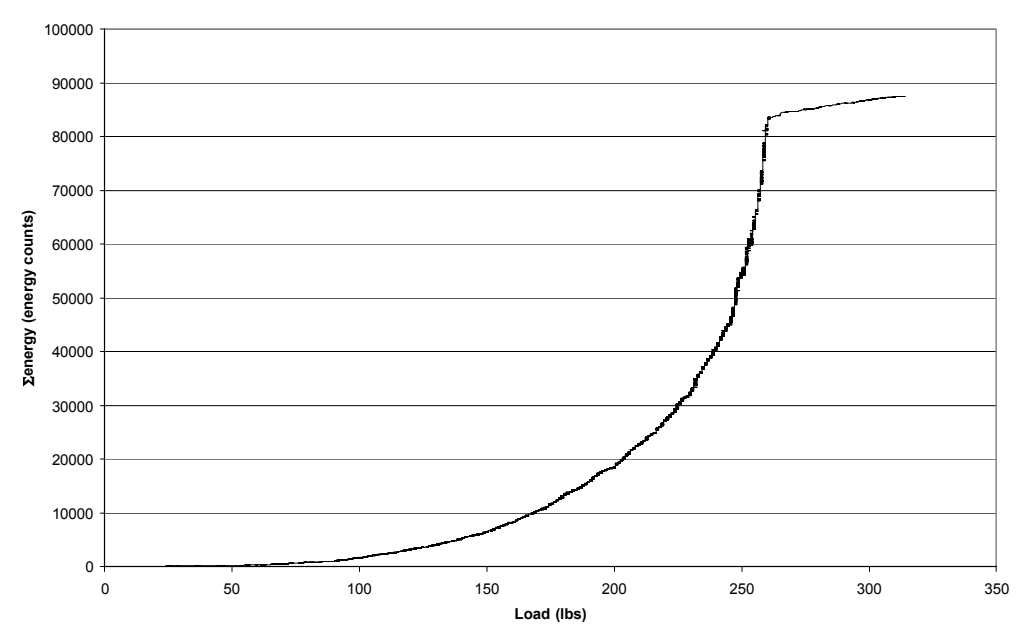

Test B9, ch 182: Eenergy vs Load

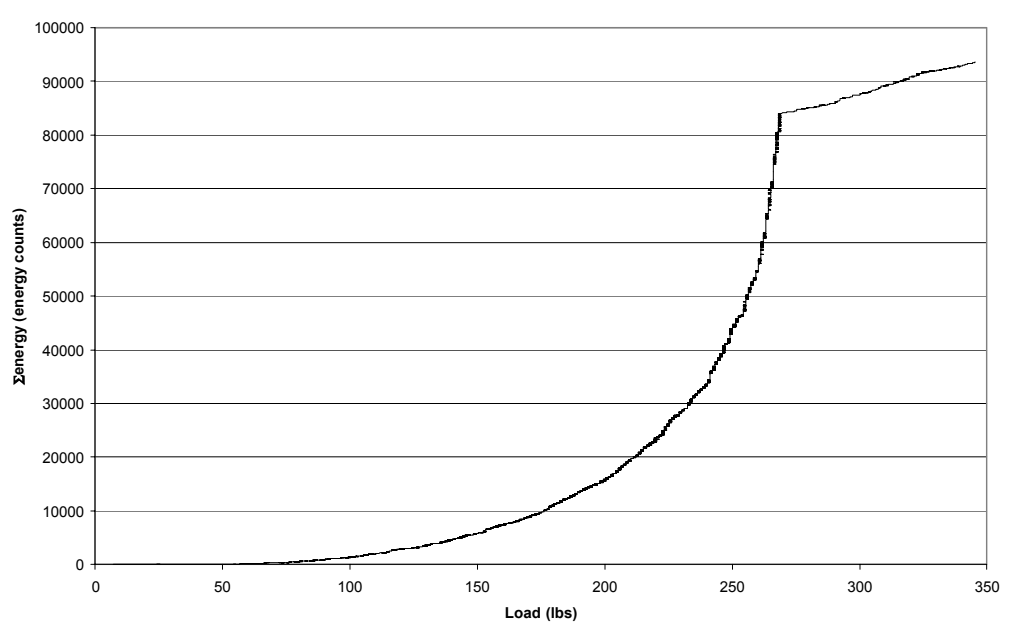

Test B10, ch 1\&2: Eenergy vs Load

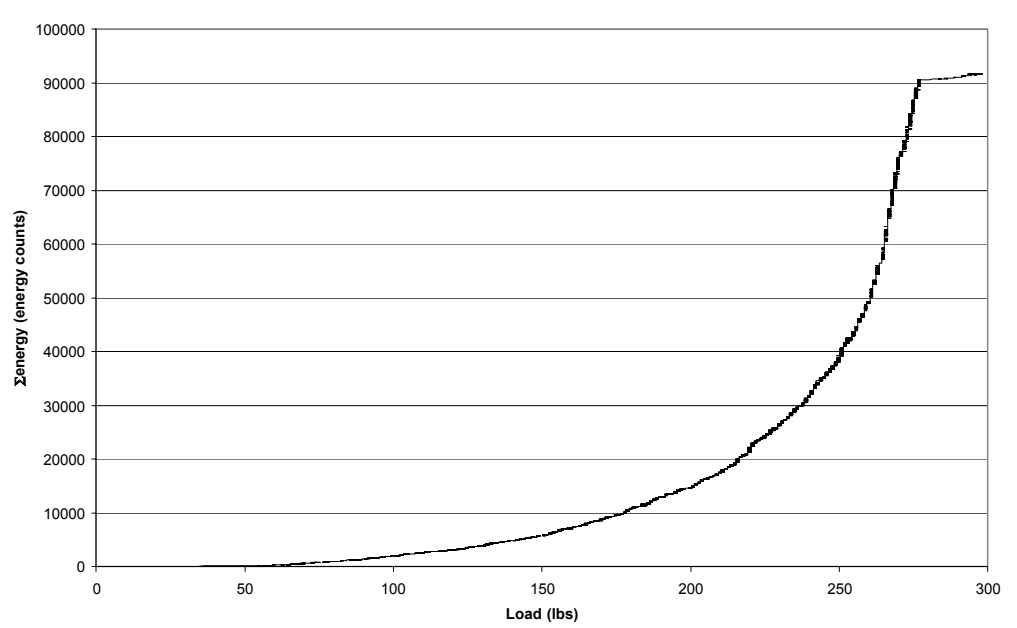

Appendix-F: $\Sigma$ Energy vs. Load Plots for Bending Specimens 
Test C1, ch 1\&2: Eenergy vs Load

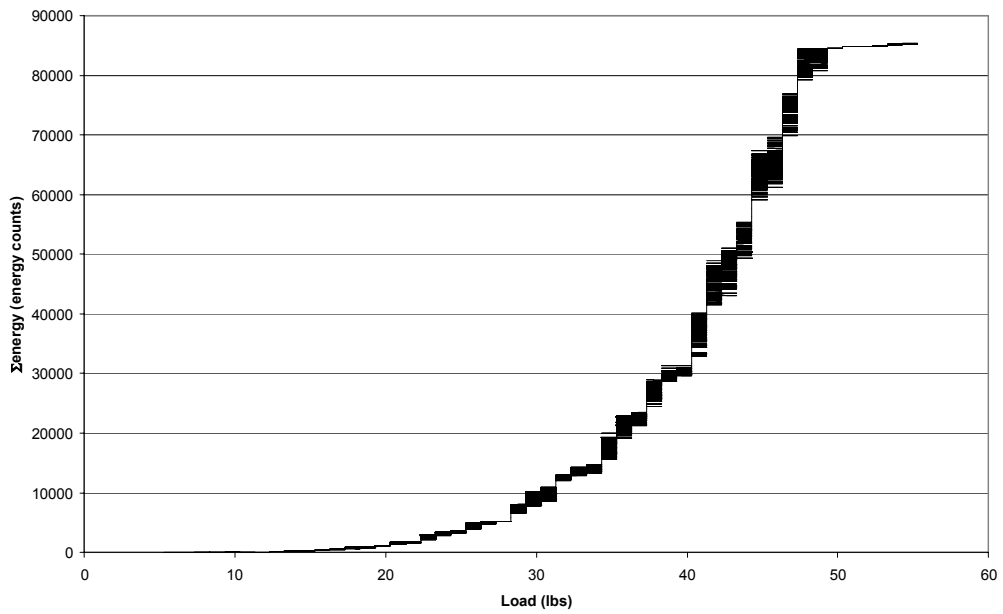

Test C3, ch 1\&2: Eenergy vs Load

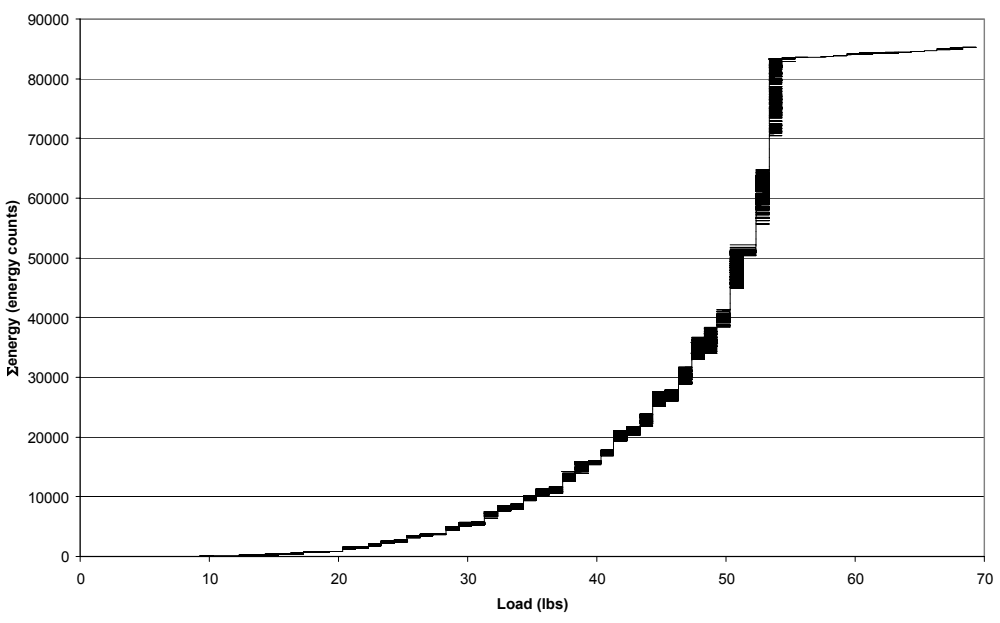

Test C4, ch 1\&2: Lenergy vs Load

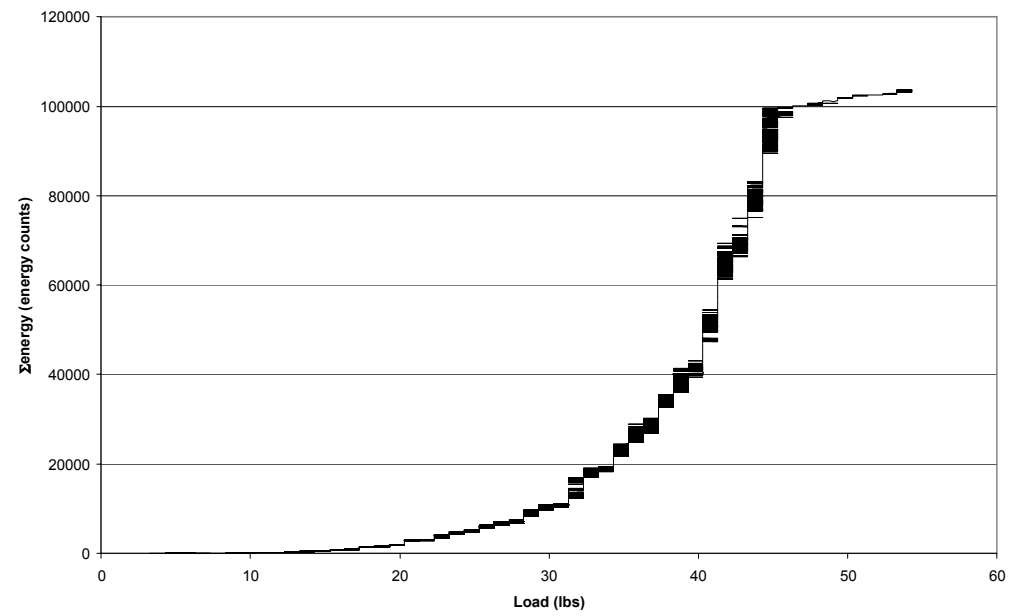

Test C5, ch 1\&2: Eenergy vs Load

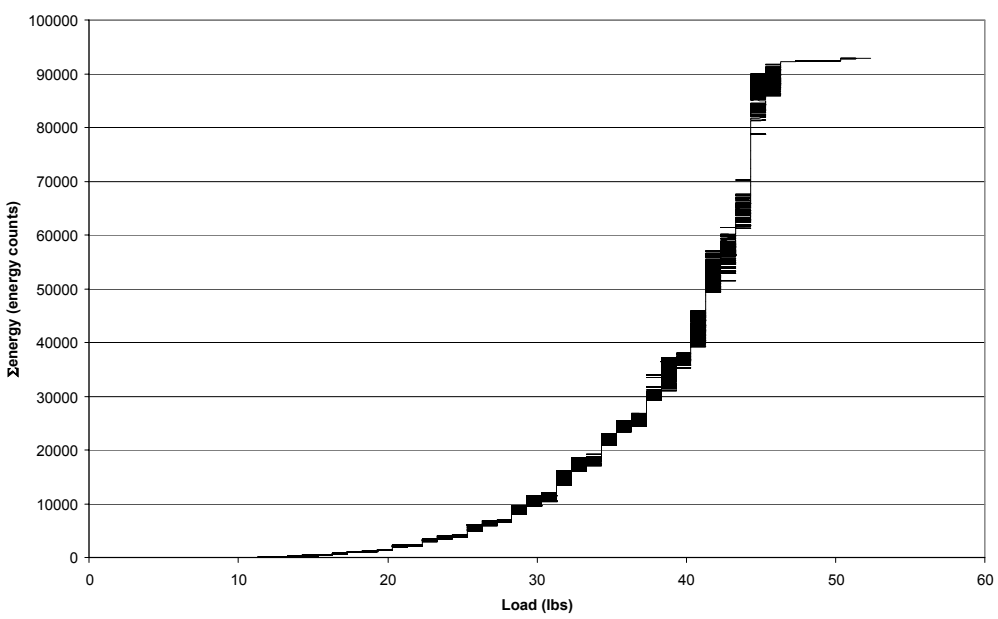

Appendix-F: $\Sigma$ Energy vs. Load Plots for Bending Specimens

180 


\section{Appendix-G}

\section{Load vs. Deflection Plots}

Bending Specimens

TestsB2-B5, B6, B8-B9, C1-C3, C5

Appendix-G: Load vs. Deflection Plots for Bending Specimens 
Test B2: Load vs. Deflection

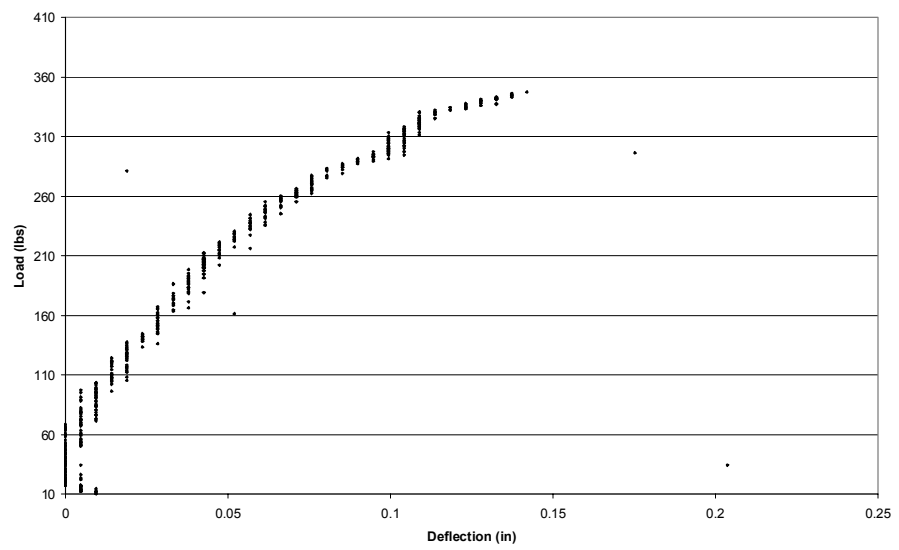

Test B3: Load vs Deflection

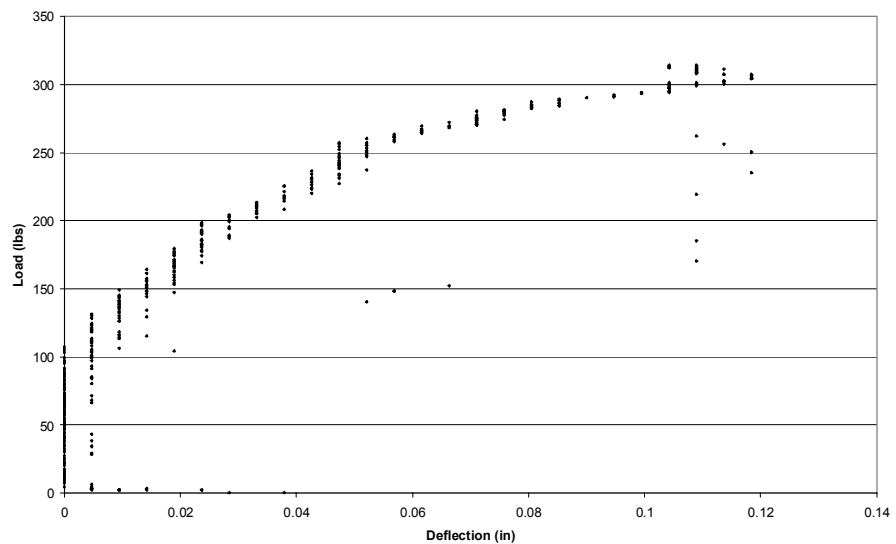

Test B4: Load vs Deflection

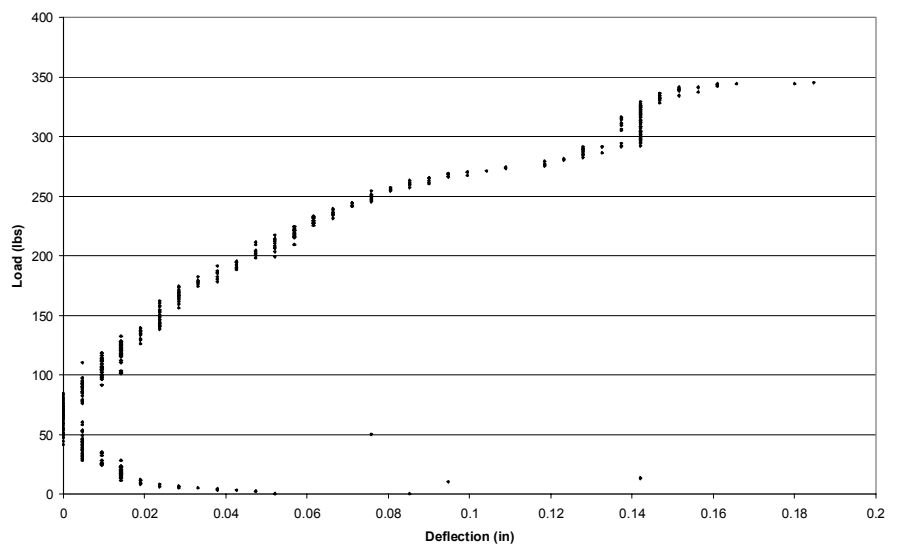

Test B5: Load vs Deflection

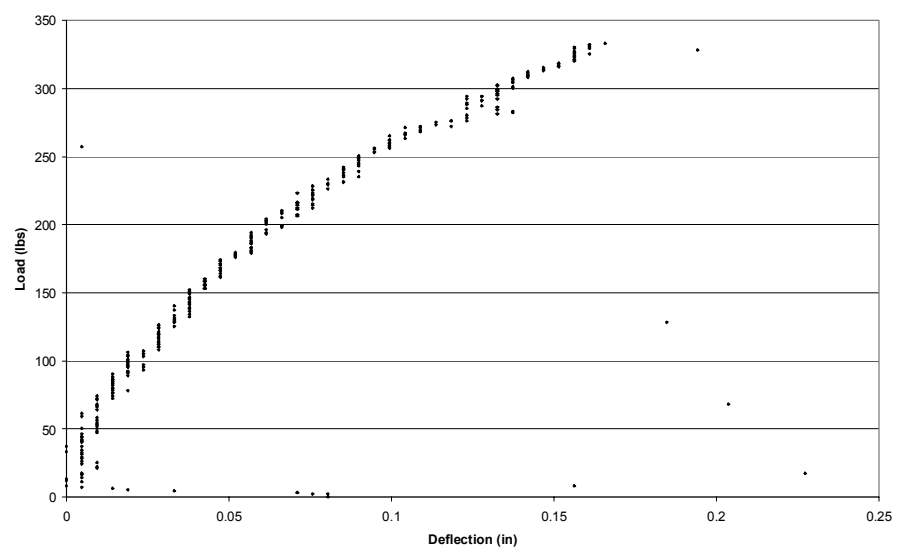

Appendix-G: Load vs. Deflection Plots for Bending Specimens

182 
Test B6: Logd vs Deflection

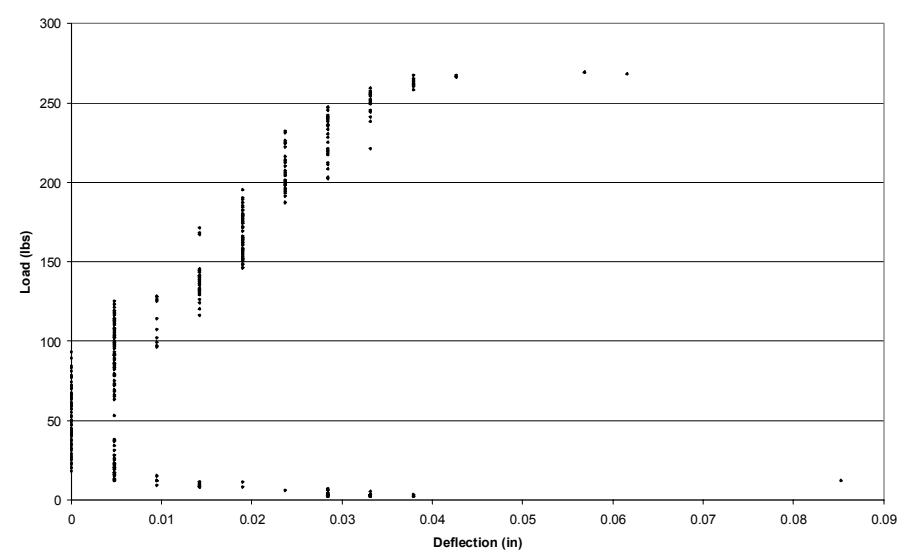

Test B8: Load vs Deflection

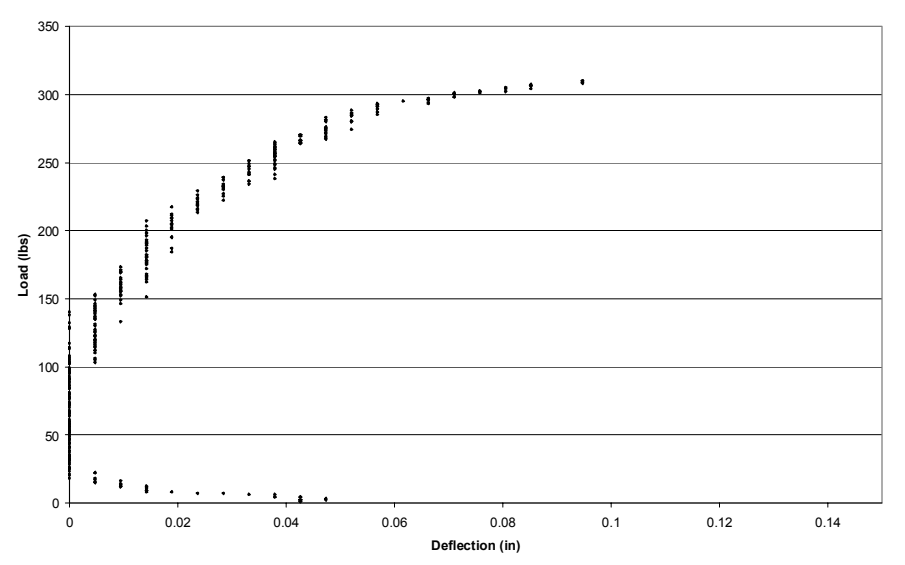

Test B9: Load vs Deflection

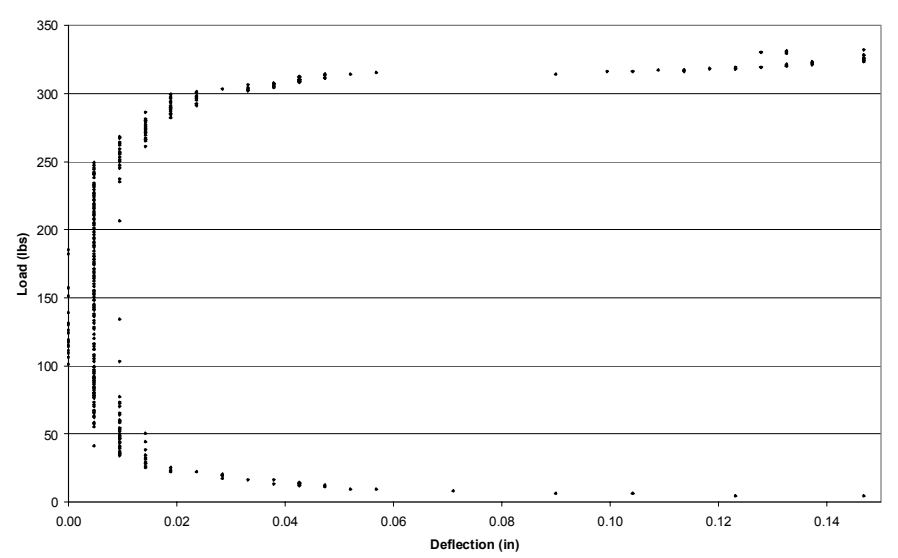

Appendix-G: Load vs. Deflection Plots for Bending Specimens 183 

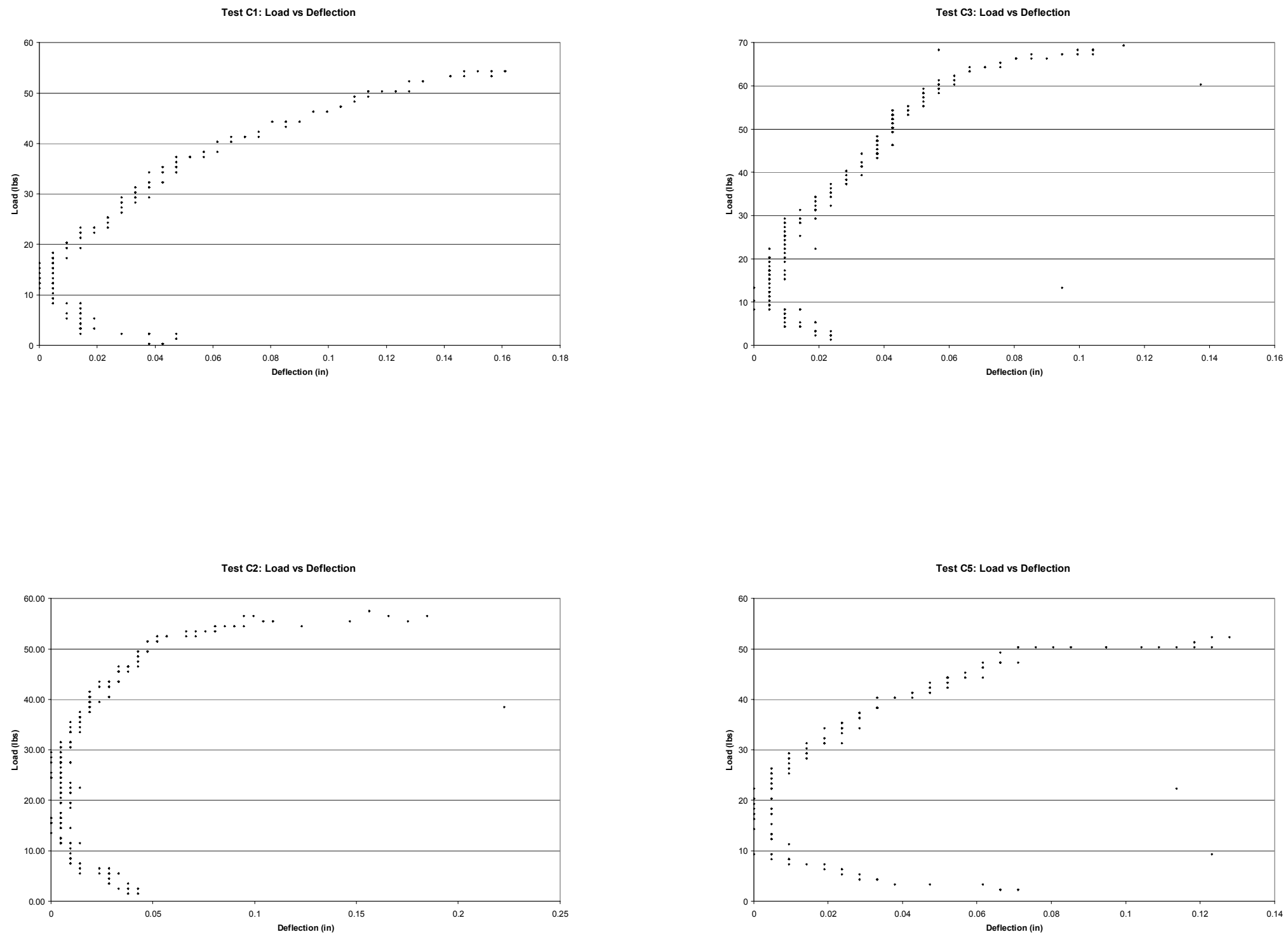

Appendix-G: Load vs. Deflection Plots for Bending Specimens

184 


\section{Vita}

Ryan Edward Arnold was born November 5, 1978 in Harrisonburg, Virginia. Raised at Cabins, West Virginia on the North Fork of the South Branch of the Potomac River, he graduated as valedictorian of the Petersburg High School class of 1997. In August 1997, he entered West Virginia University, and earned a Bachelor of Science in Civil Engineering degree in May 2001. In the same year he passed the FE exam to become an EIT. He entered the graduate program with a specialty in structures in August 2001. In May 2003 he met all requirements for the degree of Master of Science in Civil Engineering and returned home to Cabins, West Virginia to work for the West Virginia Division of Highways - District 5.

\section{$\underline{\text { Articles }}$}

- Chen, H.L., and Arnold, Ryan E., "Acoustic Emission Analysis of FRP Specimens in Tension and Bending", To Be Submitted To Materials Evaluation.

- Chen, H. L., and Arnold, Ryan E., "Evaluation of FRP Composites Using Acoustic Emissions and Neural Networks", To Be Submitted To Journal of Engineering Mechanics, ASCE. 